\title{
Automatic Calculations in High Energy Physics and GRACE at one-loop
}

\author{
G. Bélanger ${ }^{1)}$, F. Boudjema ${ }^{1)}$, J. Fujimoto ${ }^{2)}$, T. Ishikawa ${ }^{2)}$, \\ T. Kaneko ${ }^{2)}$, K. Kato ${ }^{3)}$, Y. Shimizu ${ }^{2,4)}$ \\ 1) LAPTH , B.P.110, Annecy-le-Vieux F-74941, France \\ 2) KEK, Oho 1-1, Tsukuba, Ibaraki 305-0801, Japan \\ 3) Kogakuin University, Nishi-Shinjuku 1-24, Shinjuku, Tokyo 163-8677, Japan \\ 4) Graduate University for Advanced Studies, Hayama, Miura-gun, Kanagawa, \\ 240-0193, Japan
}

†UMR 5108 du CNRS, associée à l'Université de Savoie. 


\begin{abstract}
We describe the main building blocks of a generic automated package for the calculation of Feynman diagrams. These blocks include the generation and creation of a model file, the graph generation, the symbolic calculation at an intermediate level of the Dirac and tensor algebra, implementation of the loop integrals, the generation of the matrix elements or helicity amplitudes, methods for the phase space integrations and eventually the event generation. The report focuses on the fully automated systems for the calculation of physical processes based on the experience in developing GRACE-loop which is a general purpose code applicable to one-loop corrections in the Standard Model. As such, a detailed description of the renormalisation procedure in the Standard Model is given emphasizing the central role played by the non-linear gauge fixing conditions for the construction of such automated codes. These new gauge-fixing conditions are used as a very efficient means to check the results of large scale automated computations in the Standard Model. Their need is better appreciated when it comes to devising efficient and powerful algorithms for the reduction of the tensorial structures of the loop integrals and the reduction of the $N>4$ point-function to lower rank integrals. A new technique for these reduction algorithms is described. Explicit formulae for all two-point functions in a generalised non-linear gauge are given, together with the complete set of counterterms. We also show how infrared divergences are dealt with in the system. We give a comprehensive presentation of some systematic test-runs which have been performed at the one-loop level for a wide variety of two-to-two processes to show the validity of the gauge check. These cover fermion-fermion scattering, gauge boson scattering into fermions, gauge bosons and Higgs bosons scattering processes. Comparisons with existing results on some one-loop computation in the Standard Model show excellent agreement. These include $e^{+} e^{-} \rightarrow t \bar{t}, W^{+} W^{-}, Z H ; \gamma \gamma \rightarrow t \bar{t}, W^{+} W^{-}$; $e \gamma \rightarrow e Z, \nu W$ and $W^{+} W^{-} \rightarrow W^{+} W^{-}$. We also briefly recount some recent development concerning the calculation of one-loop corrections to 3 body final states cross sections in $e^{+} e^{-}$with the help of an automated system.
\end{abstract}




\section{Contents}

1 Introduction 1

1.1 The need for automation in the Standard Model . . . . . . . . . . . . . . . . . 1

1.2 Different approaches and levels of automation . . . . . . . . . . . . . . 2

1.2.1 Automation at tree-level . . . . . . . . . . . . . . . . . 2

1.2.2 Automation at one-loop level . . . . . . . . . . . . . . . 3

1.2.3 Checking the correctness of the results . . . . . . . . . . . . . 4

1.3 Importance of judicious gauge-fixing for automated one-loop calculations . . . . . 5

1.4 Plan and outline of the review . . . . . . . . . . . . . . . . 6

\begin{tabular}{|lll}
2 & Overview of an automatic svstem:GRACE as an example & $\mathbf{7}$
\end{tabular}

2.1 Specialised codes and building blocks . . . . . . . . . . . . . . . . . . 10

2.2 Implementation of the model file $\ldots \ldots \ldots \ldots \ldots$. . . . . . . . . . 11

2.3 Fevnman Diagram Generation . . . . . . . . . . . . . . . . . . . . 13

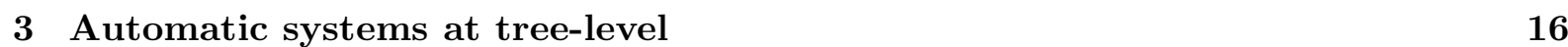

3.1 Squared matrix elements . . . . . . . . . . . . . . . . . . . . . . . 16

3.2 Spinor technique: a worked out example in GRACE . . . . . . . . . . . . . 17

3.3 Dealing with QCD and supersvmmetrv . . . . . . . . . . . . . . . . 19

3.4 Checking the result at tree-level . . . . . . . . . . . . . . . . . . . . . 21

3.5 Automatic tree calculations without Fevnman graphs . . . . . . . . . . . . . . 21

3.6 Kinematics and event generation . . . . . . . . . . . . . . . . . 21

$4 \quad$ Automatic systems for one-loop processes $\quad 22$

4.1 Gauge parameter independence . . . . . . . . . . . . . . . . . . . 24

4.2 Generation of matrix elements . . . . . . . . . . . . . . . . . 25

4.3 Regularisation scheme and the issue of $\gamma_{5} \ldots \ldots \ldots \ldots \ldots$

4.4 Size of the generated programs $\ldots \ldots \ldots \ldots \ldots$. . . . . . . . . . . . . .

$5 \quad$ The Standard Model in a general non-linear gauge $\quad 28$

5.1 The classical Lagrangian . . . . . . . . . . . . . . . . . . . . . . . . 28

5.2 Quantisation: Gauge-fixing and Ghost Lagrangian . . . . . . . . . . . . . . . 30

$6 \quad$ Renormalisation and counterterms 32

6.1 Renormalisation constants . . . . . . . . . . . . . . . . . . 32

6.2 Two-point functions at one-loop including counterterms . . . . . . . . . . . . . . 33

6.3 Renormalisation Conditions . . . . . . . . . . . . . . . . . . . 35

6.4 Some remarks on the explicit form of the renormalisation constants . . . . . . . . 38

6.4.1 Mass shifts and charge renormalisation . . . . . . . . . . . . . . 38

6.4.2 Wave function renormalisation constants . . . . . . . . . . . . . 38

6.5 Issues of renormalisation for unstable particles . . . . . . . . . . . . . . . 40

6.5.1 Wave function renormalisation for unstable particles: absorptive part and gauge dependence 40

6.5.2 Width implementation for resonant diagrams and gauge invariance . . . . 42 
\begin{tabular}{lll}
\hline & Evaluation of the loop integrals & 48
\end{tabular}

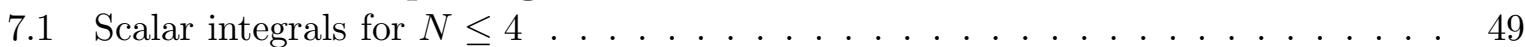

7.2 Reduction of the tensor integrals for $N<4 \ldots \ldots \ldots \ldots \ldots$

7.3 Reduction of the higher rank parametric box integrals . . . . . . . . . . . . . 52

$7.4 \log (\Delta)$ terms for the box and triangle $\ldots \ldots \ldots \ldots \ldots$

7.5 Reduction of the higher rank parametric integrals for the triangle . . . . . . . . . 54

7.6 Reduction of 5 - and 6 -point integrals . . . . . . . . . . . . . . . . 55

7.7 New techniques for the loop integrals . . . . . . . . . . . . . . . . . 58

8 Tests on the loop calculation $\quad 59$

8.1 Ultraviolet and infrared finiteness checks . . . . . . . . . . . . . . . . . 60

8.1 .1 Ultraviolet finiteness . . . . . . . . . . . . . . . . . . . . . 60

8.1.2 Infrared finiteness and calculation of the soft-bremsstrahlung factor . . . 60

8.2 Gauge-parameter independence checks . . . . . . . . . . . . . . . 61

8.3 Inclusion of hard bremsstrahlung, $k_{r}$ stability $\ldots \ldots \ldots \ldots \ldots$

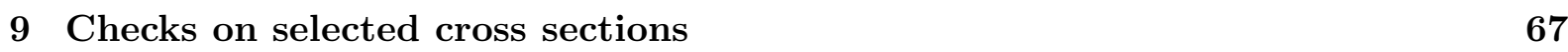

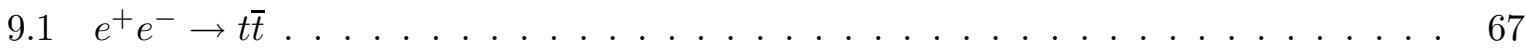

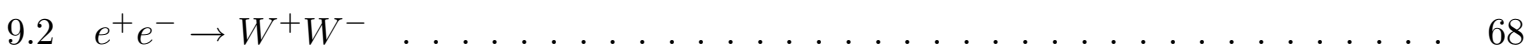

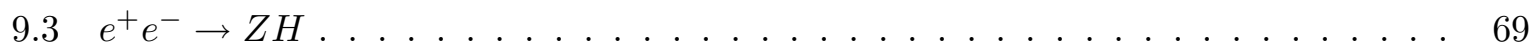

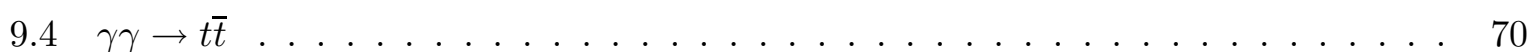

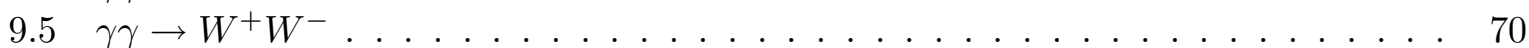

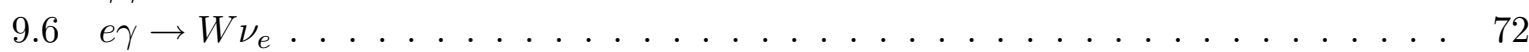

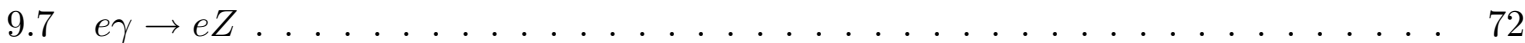

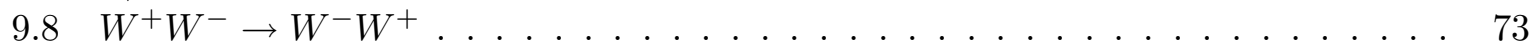

$\begin{array}{ll}10 \text { Conclusions } & 76\end{array}$

\begin{tabular}{|ll}
\hline Appendices & 79
\end{tabular}

\begin{tabular}{|ll}
\hline A Specific form of the BRST transformations & 79
\end{tabular}

\begin{tabular}{lr}
\hline B Feynman Rules & $\mathbf{8 0}$
\end{tabular}

B.1 Propagators . . . . . . . . . . . . . . . . . . . . . . 81

B.2 Vector-Vector-Vector . . . . . . . . . . . . . . . . . . . . . 81

B.3 Vector-Vector-Scalar . . . . . . . . . . . . . . . . . . . . 82

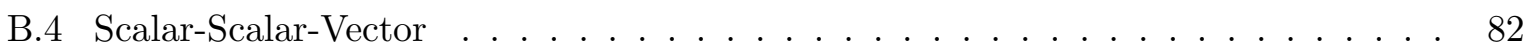

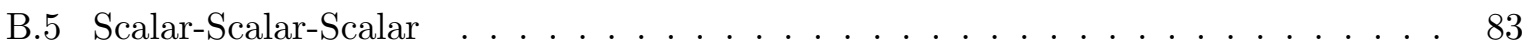

B.6 Vector-Vector-Vector-Vector . . . . . . . . . . . . . . . . . . . 83

B.7 Vector-Vector-Scalar-Scalar . . . . . . . . . . . . . . . . . 84

B.8 Scalar-Scalar-Scalar-Scalar . . . . . . . . . . . . . . . . . . . 85

B.9 Fermion-Fermion-Vector . . . . . . . . . . . . . . . . . . 85

B.10 Fermion-Fermion-Scalar . . . . . . . . . . . . . . . . . 86

B.11 Ghost-Ghost-Vecton . . . . . . . . . . . . . . . . . . . . 86

B.12 Ghost-Ghost-Scalan . . . . . . . . . . . . . . . . . . . . . . . 87 
B.13 Ghost-Ghost-Vector-Vector . . . . . . . . . . . . . . . . . . . . . . . . . . 88

B.14 Ghost-Ghost-Scalar-Scalar . . . . . . . . . . . . . . . . . . . . . . . . . . 89

\begin{tabular}{|cc}
\hline C Counterterms in the ghost sector & 90
\end{tabular}

D Auxiliary Fields and Generalised Ward-Takahashi Identities in the unphysical scalar sector

\begin{tabular}{|ll}
\hline E Renormalising the gauge-fixing functions & 93
\end{tabular}

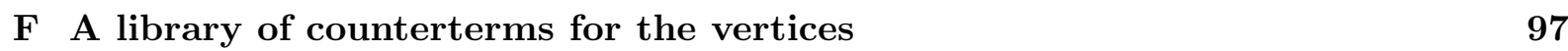

F.1 Vector-Vector-Vector . . . . . . . . . . . . . . . . . . . . 98

F.2 Vector-Vector-Scalan . . . . . . . . . . . . . . . . . . . 98

F.3 Scalar-Scalar-Vector . . . . . . . . . . . . . . . . . . . . . . 98

F.4 Scalar-Scalar-Scalar . . . . . . . . . . . . . . . . . . . . 99

F.5 Vector-Vector-Vector-Vector . . . . . . . . . . . . . . . . . . . . . . 99

F.6 Vector-Vector-Scalar-Scalar . . . . . . . . . . . . . . . . . . . 100

F.7 Scalar-Scalar-Scalar-Scalar . . . . . . . . . . . . . . . . . . . . . . 101

F.8 Fermion-Fermion-Vector ． . . . . . . . . . . . . . . . . . . . . . . 101

F.9 Fermion-Fermion-Scalar . . . . . . . . . . . . . . . . . . . . . 102

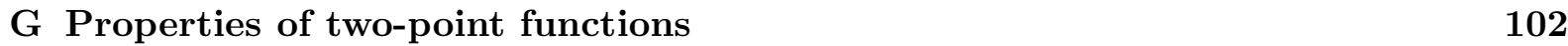

H Results for the one-loop corrections to the propagators $\quad 105$

H.1 The tadpold . . . . . . . . . . . . . . . . . . . . . . . . . 105

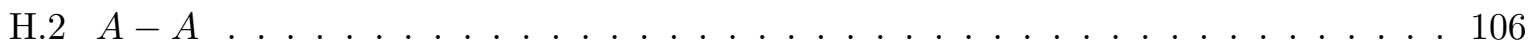

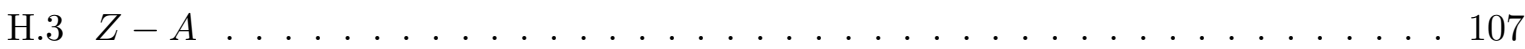

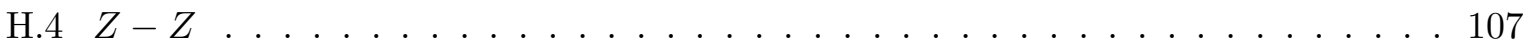

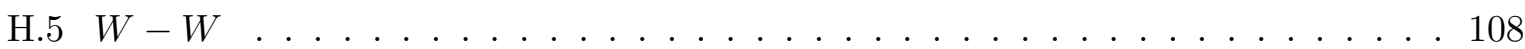

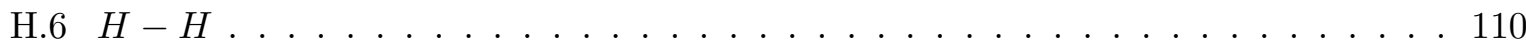

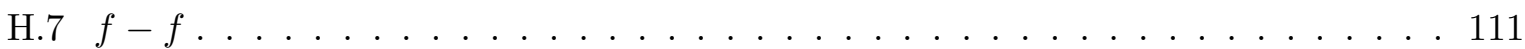

H.8 The Goldstone secton . . . . . . . . . . . . . . . . . . . . . . . . . . . 112

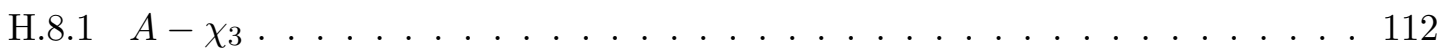

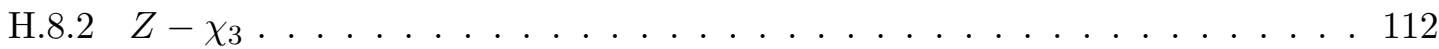

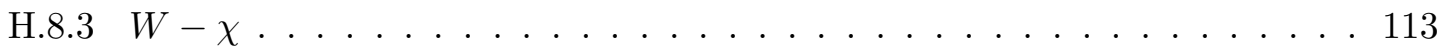

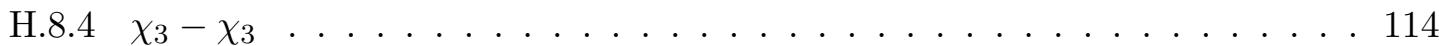

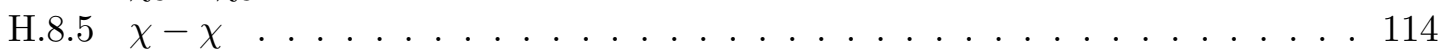

\begin{tabular}{ll}
\hline Direct determination of the charge counterterm & 115
\end{tabular}

\begin{tabular}{|lll}
\hline & Graph theory and optimization in the generation of Fevnman diagrams & 117
\end{tabular}

\begin{tabular}{lr}
\hline Bibliography & 121
\end{tabular} 


\section{Introduction}

\subsection{The need for automation in the Standard Model}

Much of the success of the Standard Model, $\mathcal{S M}$, of the electroweak interaction rests on the results of the various precision measurements, notably those of LEP and SLC. These precision measurements required the knowledge of higher order quantum corrections. Although the latter are rather involved, calculations are still under control since the bulk of the observables pertain to two-body final states. In fact due to the present available energy, the most precise predictions relate to fermion pair production, a calculation which is far easier to handle than that for $W$ pair production even if one leaves out the fact that for the latter one needs a full 4 -fermion final state calculation. Next generation machines will involve much higher energies and luminosities opening up the thresholds for multiparticle production and/or the need to go beyond one and two-loop radiative corrections. On the other hand even when one only considers three particles in the final state, the complexity increases tremendously especially within the electroweak framework. So much so that even a process like $e^{+} e^{-} \rightarrow \nu_{e} \bar{\nu}_{e} H$ which would be the main production mechanism for the Higgs at the next linear collider and where the tree-level calculation receives a contribution from only a single (non-resonant) diagram, a full one-loop calculation has only very recently been completed [1, 2, 3]. For such processes, hand calculations become quickly intractable and very much prone to error. Moreover, a complete hand calculation for such processes is not possible, even for the tree-level cross sections, as one has to resort to numerical methods for the phase space integration. Especially for QCD processes, to alleviate some of the major hurdles in the calculation of matrix elements for physical observables beyond leading and next-to-leading order, one has devised some powerful alternatives to the standard diagrammatic Feynman approach [4, with most recently the development of the twistor-space [5, 6, 7, 8. However most of them, if not all, involve at most one massive particle and a single parameter, the QCD coupling constant. Moreover the techniques work because of the exact gauge symmetry of QCD and thus, apart from a handful processes, these methods can not be carried over to the electroweak theory where the computations involve a variety of masses and scales. Faced with these difficulties the need for computers is even more evident for the calculation of electroweak processes.

Ideally one would like to automatise the complete process of calculating radiative corrections and multi-particle production starting from the Lagrangian or the Feynman rules to the cross section. Automation is, in principle, feasible since most of the ingredients of perturbation theory are based on well established algorithms. With the increase in computer power and storage, together with possible parallelization, one could deal, in a relatively short time, with more and more complex projects thus bypassing the problem of huge output files being produced, at least in the intermediate stages. The idea of automation of the calculations in high-energy physics is not new. It dates back to the 1960's when packages such as SCHOONSCHIP 9 and then REDUCE [10, 11] had been developed. These are symbolic manipulation codes that automatise the algebraic parts of a matrix element evaluation, like traces on Dirac matrices and contraction of

Lorentz indices. Such codes have evolved quite a lot with applications not only optimised for high-energy physics like FORM[12] but also more general purpose programs like Mathematica[13. and Maple 14. Generation of QED Feynman graphs at any order in the coupling constant 
was automatised in the late 70's 15. One of the first major application of these early developments in this field was the calculation of the anomalous magnetic moments of the electron and the muon[16]. The first automatic system incorporating all the steps for the calculation of a cross section, from Feynman graph generation, amplitude generation through a REDUCE source code that produces a FORTRAN code, phase space integration and event generation with BASES/SPRING[17] is GRAND[18. It was limited to tree-level processes in QED. In the early nineties, a few groups started to develop packages aiming at the automatisation in the $\mathcal{S M}[19$.

\subsection{Different approaches and levels of automation}

A hand calculation of a process at a certain order in perturbation can follow different methods, approaches, tricks and sometimes relies on approximations. It is no wonder then that these same issues and variations have translated into the automation of these calculations and have led to the implementation of a few softwares with varying degrees of automation, different domain of application while exploiting different programming languages, see Ref. [20] for a survey of some of these systems. Some of the systems are collections of software tools used to help only certain aspects of the hand calculation. Example are codes that only generate the Feynman diagrams, like QGRAF 21] or codes for the algebraic and analytic manipulations on loop diagrams but on a diagram by diagram basis like XLOOPS 22. Others are designed for specific applications 23, 24, like QCD corrections to some electroweak processes for example. The report will concentrate only on the fully automatised systems that are able to output a source code for the numerical calculation of cross sections without any intervention by the user, apart of course from providing the input which consists in specifying the process. In reviewing the characteristics of these codes and the different steps that go into building these tools, we will see that some of the specialised codes we have just mentioned could be considered as a module in the long chain that goes from the Lagrangian to the cross section. To go into the details of how the various steps are implemented we will have to be more specific, since there is hardly any standardisation of either the methods, the algorithms or the computer language. Therefore we will most of the time refer to the experience we gained in developing GRACE. We therefore present the case of automation in the $\mathcal{S M}$ at the tree-level and concentrate more on the one-loop level. A fully automatic system beyond one-loop has not been constructed yet.

\subsubsection{Automation at tree-level}

In the usual diagrammatic Feynman approach followed by most of the automated systems, the cross sections can be obtained by computing directly the unpolarised squared matrix elements or in terms of the (helicity) amplitudes using spinor techniques. Although the computer algorithms for these two techniques can be quite different, in both cases one needs the Feynman rules and Feynman graphs to be generated. The automatic systems, GRACE[25], CompHEP 26, the FeynArts-FeynCalc-FormCalc package 27, 28, 29, 30, 31, Madgraph 32, and fdc 33, follow the diagrammatic approach with applications to both the $\mathcal{S M}$ and its supersymmetric version. A detailed description of the Minimal Supersymmetric Standard Model, $\mathcal{M S S M}$, of GRACE is found in 34].

It is also possible to make do without Feynman diagrams and arrive even more directly at the 
cross sections. This can be achieved through an iterative solution of the equations of motion or by solving the Dyson-Schwinger equation 35, 36, 37. This approach leads to faster codes. However, it has not been extended beyond tree-level. Two 25, 29] of the diagrammatic approach codes have on the other hand been extended to one-loop.

Depending on the method in the diagrammatic approach, Dirac matrices and spinors can be treated as symbolic objects before being converted to numerical quantities with the help of a symbolic manipulation system as will be explained in section 3.1. One can also take a more "numerical" approach where these objects are combined into numerical entities. This is treated in section 3.1 .

Even at tree-level, one problem is the size of the output file when one is dealing with multi-leg processes. Automatic systems produce, for a complicated process, a huge source code ${ }^{1}$ which in turn requires a large amount of CPU time for the numerical evaluation. This necessitates a large disk space, a human control over a large number of batch jobs or could even necessitate to split the source code into small pieces in order to make compilers work. In addition to the problem of size, it may be necessary to write specific kinematics routines when the amplitude has a complicated structure of singularities, see section [3.6. Of course, the non-diagrammatic approach also requires a proper phase space integration routine.

\subsubsection{Automation at one-loop level}

The problem with the size of the output files and the integration over phase space are exacerbated for one-loop processes. These are however not the major hurdles for extending a tree-level code to one-loop. One first needs to master all the theoretical background related to the renormalisation of a model or a theory. A consistent renormalisation procedure that gives all possible counterterms, which would have to be implemented in the automatic code to tackle any one-loop process, needs to be clearly defined. A symbolic treatment of space-time dimension is inevitable for the regularisation of ultraviolet divergences. Infrared divergences will have to be regularised either by a small fictitious mass or through dimensional regularisation, DR, 38, 39. A major investment has to do with a fast and efficient algorithm for the loop integrations, especially the algorithm for the reduction of the tensorial structures to the scalar $N$-point functions and the reduction of the $N>4$ scalar functions to lower $N$-point functions for codes that allow multi-leg one-loop integrals. Here also, almost each code reverts to a specific technique and algorithm. For one-loop calculation in the electroweak theory, $2 \rightarrow 2$ processes are now easily and fully automatised as will be made evident in the report. Although there is a large number of Feynman graphs for $2 \rightarrow 3$ processes, automatic systems such as the package FeynArts-FeynCalc-FormCalc with the extension [40] of the one-loop library LoopTools [41] or GRACE-loop have shown the feasibility of an automatic one-loop calculation for $2 \rightarrow 3$ processes in the $\mathcal{S M}$, where human intervention is kept to management of the large number of files and batch jobs. Most important processes for Higgs production at the linear collider, $e^{+} e^{-} \rightarrow \nu_{e} \bar{\nu}_{e} H$ [1, 2, 3], $e^{+} e^{-} \rightarrow e^{+} e^{-} H$ [42], $e^{+} e^{-} \rightarrow Z H H$ [43, 44, $e^{+} e^{-} \rightarrow t \bar{t} H$ [45, 46, 47], $\gamma \gamma \rightarrow t \bar{t} H$ [48] as well as $e^{+} e^{-} \rightarrow e^{+} e^{-} \gamma$ [49] have been computed thanks to the automatic systems. This is also the case of the most re-

\footnotetext{
${ }^{1}$ The size of the problem grows rapidly as the number of external particles increases. For example, $2 \rightarrow N$ tree process in $\phi^{3}$ model has $(2 N-1)$ !! Feynman graphs.
} 
cent calculations of the complete one-loop electroweak corrections to $2 \rightarrow 4$ processes, namely $e^{+} e^{-} \rightarrow \nu \bar{\nu} H H[50]$ and some specific 4-fermion channels in $e^{+} e^{-} \rightarrow 4 f[51]^{2}$.

\subsubsection{Checking the correctness of the results}

An automatic system produces some numbers as the results of a black-box calculation which may not necessarily be correct. A user may feed some input data which the authors of the system have not thought of. There may be bugs in the program which have not been detected with the tests made by the developers. Compilers may have problems, especially with highly optimizing options. Even if the generated program is logically correct, numerical cancellations may produce wrong results. The Monte-Carlo integration package may give some false value just because of insufficient sampling points. Thus systematic procedures of checking the results are indispensable for automatic systems. These procedures will be classified into three categories:

i) Checks by the computer system

If the generated code is set-up so that it can be run with different accuracies (double, quadruple precision), it would be easy to detect problems related to numerical cancellations. Good FORTRAN compilers supply options for changing precisions without modification of the source code. It is also a good idea to run the program on other machines with different compilers or architecture. For the Monte-Carlo integration, one can increase the number of sampling points in order to test the stability of the results.

ii) Self-consistency checks within the automatic system

If the theoretical model has a free parameter, related to its symmetry, which does not alter the physical results, it will be used to check the results. Physical quantities in gauge theories are independent under the change of gauge parameters. When an automatic system includes gauge parameters as variables in the generated code of the numerical calculation, one can explicitly check the gauge invariance of the obtained results. When the system keeps the regularisation parameters of the ultraviolet or infrared divergences, one can confirm the cancellation of these divergences explicitly. The incorporation of these self-checking procedures is one of the most important feature for the reliability of the automatic calculation.

iii) Comparison with other calculations

This is a standard procedure provided another independent calculation exists or can be performed using a different automatic code.

Although the ultraviolet and infrared tests are rather straightforward to implement, the gauge parameter check requires a very careful and judicious choice of the gauge-fixing function. This is especially true in automatic codes for one-loop amplitudes and cross sections. A few tree-level codes have the gauge parameter check incorporated through the usual linear 't HooftFeynman gauges or give the possibility to switch to the unitary gauge. For example, in GRACE and for tree-level processes, the gauge-parameter independence check has been applied successfully by comparing the results in the unitary gauge to those of the 't Hooft-Feynman gauge. An

\footnotetext{
${ }^{2} \mathrm{~A}$ first investigation of these processes using an automatic code was done in 52 .
} 
agreement up to $\sim 15(\sim 30)$ digits in double (quadruple) precision has been reached for a few selected points in phase space therefore confirming, at this stage, that the system works very well for tree-level processes. However, as we will see, these types of gauges are not suited at all for one-loop calculations in the electroweak sector and explain, in part, why there are at the moment only two general purpose codes for one-loop calculations. None of them exploits or is defined for a general linear $R_{\xi}$ gauge. The latter tends to considerably increase an already very large size of the file corresponding to each of the numerous one-loop diagrams. Not only the expressions get large compared to the usual $\xi=1$ 't Hooft-Feynman gauge but also call for extending the algorithms for the reduction of some new tensor and $N$-point function integrals. These are at the heart of a one-loop calculation especially that their evaluation is very much time consuming. Generalised non-linear gauges [53, 54], still defined with the gauge parameter $\xi=1$, are on the other hand very well suited for an automatic code of one-loop amplitudes. This will become clear when we will go through the different stages of a one-loop calculation and the different modules that are required for the construction, or the extension to, one-loop amplitudes in the electroweak theory. The implementation of the generalised non-linear gauge is therefore crucial in GRACE at one-loop. This is also the reason it has a quite central place in this report and deserves that we summarise, already at this stage, some of its salient features and the simplifications it brings when implemented in an automatic code for one-loop processes. Note that the package FeynArts-FeynCalc has the $\mathcal{S M}$ defined in the background-field gauge 55 beside the usual linear $\xi=1$ Feynman gauge.

\subsection{Importance of judicious gauge-fixing for automated one- loop calculations}

A computation in a general $R_{\xi}$ gauge or unitary gauge brings about unnecessary complications and sometimes troublesome numerical unstabilities especially when one deals with several gauge bosons. Take for instance the propagator of the $W$ gauge boson, of momentum $k$ and mass $M_{W}$. In a general $R_{\xi}$ gauge, with the gauge fixing parameter $\xi_{W}$, it writes

$$
\frac{1}{k^{2}-M_{W}^{2}}\left(g_{\mu \nu}-\left(1-\xi_{W}\right) \frac{k_{\mu} k_{\nu}}{k^{2}-\xi_{W} M_{W}^{2}}\right) \text {. }
$$

In the 't Hooft-Feynman gauge, $\xi_{W}=1$, only the "transverse" part consisting of the metric tensor $g_{\mu \nu}$ contributes and leads to a straightforward contraction of neighbouring vertices. Numerical instabilities are due to the contribution of the "longitudinal" $k_{\mu} k_{\nu}$ part of the gauge propagators. Moreover, the longitudinal tensor structure considerably inflates the size of each intermediate result, for example with $n$ intermediate heavy gauge bosons instead of performing $n$ operations one performs $2^{n}$ operations. Since the longitudinal expressions involve momenta, they can contribute terms that increase with energy and which require a subtle cancellation among various diagrams. A situation which is most acute in the unitary gauge, obtained by formally taking $\xi_{W} \rightarrow \infty$ in Eq. 1.1. These problems are of course exacerbated in loop calculations and, as is known, calculations and renormalisability itself are arduous if not problematic in the unitary gauge [56]. Within GRACE one of the problems in these gauges (unitary, or general linear type gauges) is that the library containing the various loop integrals is designed assuming that the 
numerator for the propagator of the vector particles is $g^{\mu \nu}$. For instance, the library for the three-point vertex functions is implemented with only up-to the third-rank tensor and therefore the library applies equally well with fermion loops, gauge loops or a mixture of these if the calculation is performed in the 't Hooft-Feynman gauge. In any other gauge one would have, for the vertex functions alone, had to deal with a 9th rank tensor! Again this not only creates very large expressions but also introduces terms with large superficial divergences that eventually need to be canceled extremely precisely between many separate contributions. Fortunately one can also work with a class of gauge-fixing conditions that maintain all the advantages of the usual 't Hooft-Feynman gauge with exactly the same simple structure for the gauge propagators. The new gauge parameters modify some vertices involving the gauge, scalar and ghost sector and at the same time introduce new vertices. In fact by judiciously choosing some of these parameters the structure of the vertices can get even simpler than with the usual linear gauge-fixing conditions. The class of gauges we are referring to exploit non-linear gauge fixing conditions [53, 54. Apart from the possible simplifications that these gauges bring, we have considered a generalised class of non-linear gauges so as to perform in an efficient way the gauge-parameter independence checks within the GRACE system. Actually the generalised gauge we choose depends on 5 parameters [54. Therefore not only this allows for a wide range of checks but since the different parameters affect different parts of the bosonic sector one can check different classes of contributions to a single process and thus more easily track down any bug. There are other welcome features of these checks. They serve as powerful tools on every step of the automated computation, from the correct implementation of the model file which in fact can be checked mostly at tree-level to the correct implementation of the tensor integrals. The reduction of the latter to scalar integrals is most prone to error. We will show how the tensor reduction is carried out in GRACE. The gauge check allows therefore to test that the reduction of these integrals into the scalar integrals is implemented properly [2]. Additional tests like those of infrared finiteness further verify the scalar integrals. Another advantage of the non-linear gauge checks over those that may be attempted within a linear $R_{\xi}$ gauge is that on a diagram by diagram basis, the gauge-parameter dependence in our checks are polynomials in the non-linear gauge parameters whereas in the linear $R_{\xi}$ gauge the dependence is buried within logarithms and rational functions. We will show how one can exploit this fact for a very powerful gauge check.

\subsection{Plan and outline of the review}

The aim of this paper is to describe in some detail the workings of a code for the automatic calculation of cross sections at one-loop in the $\mathcal{S M}$ based on the Feynman diagrammatic approach. Though a few codes will be reviewed, the details and algorithms that enter the construction of such codes are based primarily on the experience we gained while developing GRACE. The next section, section 2. will first give a general overview of the main building blocks of a generic automated package for the calculation of Feynman diagrams starting from a Lagrangian down to an integrated cross section. A short review of some specialised purpose software is given in section 2.1. We then discuss in some detail how the model file is implemented in 2.2 and how Feynman graph generation is achieved automatically in 2.3. Amplitude generation at treelevel, both through the spinor technique and directly through the squared matrix, is described 
in section 3. This section will also very briefly present alternative automatic codes that make do without Feynman graphs at tree-level. It is followed by the implementation of phase space integration and event generation. The extension to a one-loop automatic system is described in section 4. The issue of the large size of the generated code especially at one-loop and file management is discussed in this section. Section 4 will also emphasise the importance of internal self-checks on the correctness of the results of an automatic code, in particular the importance of the non-linear gauge. This naturally leads the way to two chapters serving as the theoretical background. First, in section [5 quantisation in a non-linear gauge is briefly outlined while a detailed description of the renormalisation procedure in the $\mathcal{S M}$ within a non-linear gauge is exposed in section 6. Explicit formulae for all two-point functions in a generalised non-linear gauge are given, together with the complete set of counterterms. We will also point out some of the issues of renormalisation and gauge dependence when dealing with unstable particles and the problems with the implementation of the width of these particles in section 6.5. All of this is needed for the implementation of the model file in the $\mathcal{S M}$. The need for a non-linear gauge is better appreciated when it comes to devising efficient and powerful algorithms for the reduction of the tensorial structures of the loop integrals and the reduction of the $N>4$ point-function to lower rank integrals. A new technique for these reduction algorithms is described in section 7 Section $[8$ is devoted to how the ultraviolet and infrared finiteness checks are dealt with in the system. In this same section we also give a comprehensive presentation of some systematic testruns which have been performed at the one-loop level for a wide variety of two-to-two processes to show the validity of the gauge check. These cover fermion-fermion scattering, gauge boson scattering into fermions, gauge bosons and Higgs bosons scattering processes. Section 9 is a convincing testimony of the power of a fully automatic system at one-loop since comparisons with existing results on some one-loop computations in the $\mathcal{S M}$ show excellent agreement. These include $e^{+} e^{-} \rightarrow t \bar{t}, W^{+} W^{-}, Z H ; \gamma \gamma \rightarrow t \bar{t}, W^{+} W^{-} ; e \gamma \rightarrow e Z, \nu W$ and $W^{+} W^{-} \rightarrow W^{+} W^{-}$. The final section, section [10, contains our conclusions. We also provide some detailed appendices. In particular we provide the full set of Feynman rules within the generalised non-linear gauge as well as the library for the counterterms. Full results for all the self-energy diagrams of all the particles in the model including the Goldstone sector is also relegated to an appendix.

\section{Overview of an automatic system: GRACE as an example}

The different components and steps that go into the calculation of a cross section, or even the corresponding Monte-Carlo event generator, in a code for the automatic evaluation of Feynman diagrams are depicted in Fig. 1. Automatic systems not based on the Feynman diagrammatic approach have a different architecture, moreover the technique they are based on have not been extended beyond the tree-level. We take GRACE-loop as an example. Although not all the modules are present in all such codes, especially as concerns the one-loop part, this should give an overall view of how such systems work and what inputs are required to make them function. Details of the different parts and components of the packages will be reviewed in the next sections together with the theoretical background. In the following, the most important modules 
Figure 1: GRACE System Flow.

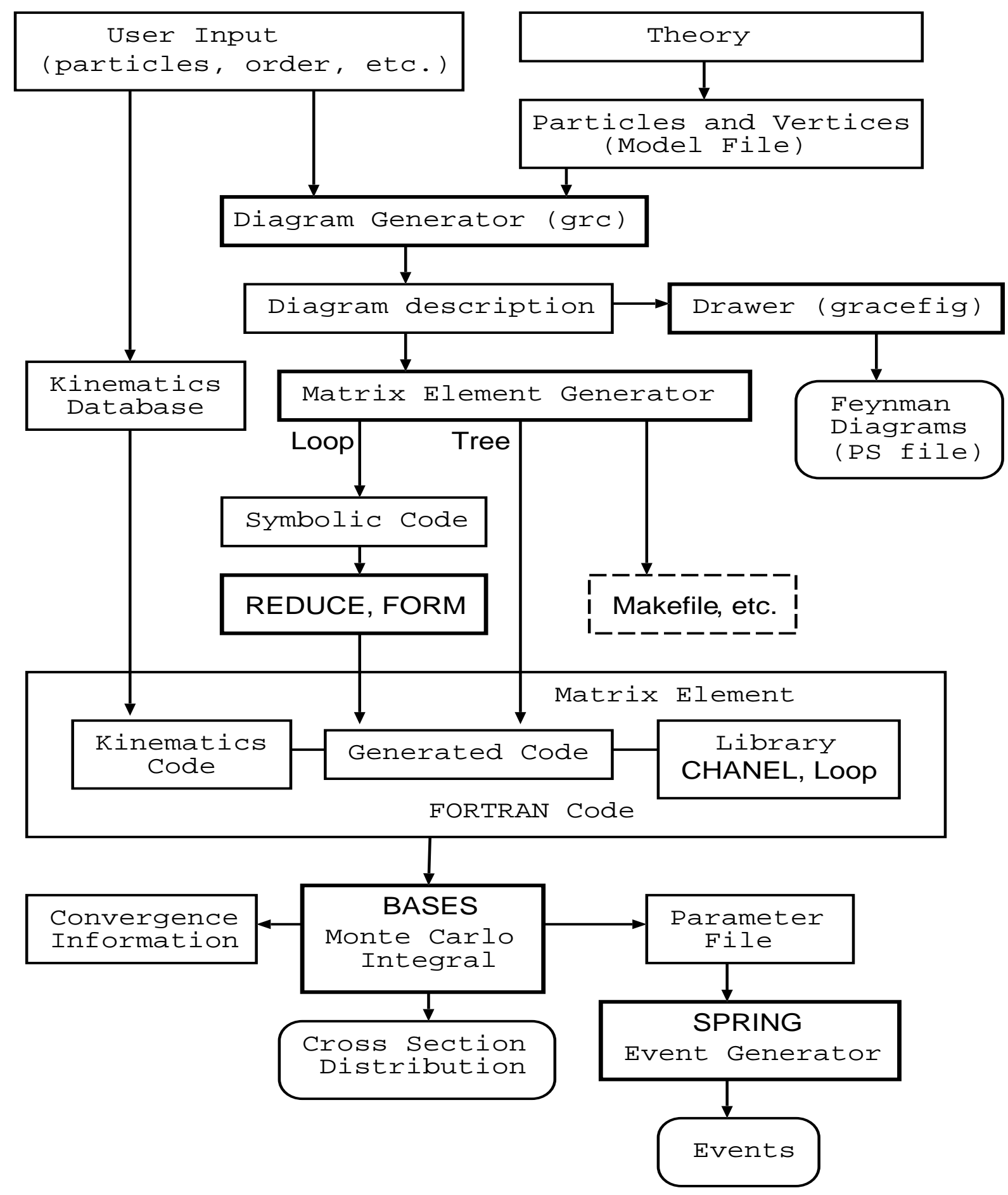


will be enclosed in boxes for easy reference to the flow chart of Fig. 1.

Theory Particles and Vertices

The system first requires the implementation and definition of a model through a model file that gives the particle content, the parameters as well as the vertices as derived from the Lagrangian. The implementation of the model file is described in more details in section 2.2

User Input

The user, on the other hand, sets as input the incoming particles and the final particles and specifies the order, in perturbation theory, at which the cross section is to be calculated.

Diagram Generator Diagram Drawer

GRACE will first generate, through its own routine grc the full set of diagrams (tree and loop as well as counterterms for the latter) with the possibility of a Postscript output of the Feynman diagrams with the help of the utility diagram-drawer gracefig. Most of the postscript files for the Feynman graphs in this report have been produced by gracefig. The FeynArts 28 package based on Mathematica performs similar steps. Section 2.3 will review some of the issues and part of the algorithms for the automatic generation of the Feynman diagrams while appendix 』 will give some more technical details about graph generation.

\section{Matrix Element Generator}

The matrix element generator encodes all the information on the diagrams. For the tree-level process the system generates a FORTRAN code which describes the helicity amplitudes using the CHANEL library and routines [57. More details about the algorithm together with a worked-out example will be presented in section 3 . We will only briefly describe the approach that avoids Feynman diagrams altogether in section 3.5.

For the computations at one-loop, one first generates a symbolic manipulation source code, based on REDUCE[11] or FORM[12] that writes, for each set of tree and loop diagrams, the interference term $\mathcal{T}_{i}^{\text {loop }} \mathcal{T}_{j}^{\text {tree }}$. A helicity formalism option is also possible here. Only then the FORTRAN source code is generated and the cross section computed with the help of the loop library and the counterterm library that performs the integration over the Feynman parameters and takes into account the counterterm constants. The symbolic manipulation for the loop calculation performs a number of important tasks, such as Dirac algebra (taking traces for fermion loops) and tensor manipulations in $n=4-2 \epsilon$ dimension if DR [38, 39] is used $^{3}$, introducing Feynman parametric integrals, shifting loop momenta appropriately etc.. FeynCalc/FormCalc 29, 30, 31] carries out a similar function with the help, for the one-loop integrals, of the LoopTools library [1].

The implementation of all these intermediate steps that are necessary at one-loop is discussed in more detail in section 4. To fully appreciate the issues at stake, it is important also to refer to the theory sections on renormalisation, section [6] and also on the algorithm for the loop integrals in section 7. As already mentioned the latter module is a critical part of an automatic code at one-loop. This also explains why almost every code comes with its own solution to the

\footnotetext{
${ }^{3}$ Some issues related to regularisation and the treatment of $\gamma_{5}$ are discussed in section 4.3
} 
problem. The implementation of this part has a bearing on the size and hence performance of the system, see section 7 and section 4.4. This calls for parallelisation and vectorisation of the codes as discussed in section 7

Phase space integration

The integration over phase space is carried via BASES [17, a Monte-Carlo integration package. One can test the convergence of the integration routine and get cross sections and distributions. The simulation and event generation is done through the package SPRING [17]. GRACE includes a number of kinematics routines, through a kinematics database, for processes with up to 6 particles in the final state. The user can select the appropriate kinematics routine from the available library depending on the singular behaviour of the process. This singular behaviour can be due to a $t$-channel photon exchange for example or some other peaking behaviour like the crossing of a resonance. Some of the issues that need to be addressed in the code for the automatic calculations of cross sections as regards integration over phase space will be described in section 3.6 .

\subsection{Specialised codes and building blocks}

As mentioned earlier, some specialised codes exist that only tackle one of the steps above. QGRAF 21] is a very powerful Feynman diagram generator. Most of the codes however are matrix element generators that work once a specific diagram is supplied to the code. Examples include MINCER 24], MATAD 23] and SHELL2 [58], all using FORM as a symbolic language. Though being devised for up to three-loop diagrams, their domain of application is limited to graphs with a restricted hierarchy of masses and momenta. Xloops 22 treats more general graphs, up to two-loop, but again on a diagram-by-diagram basis. GEFICOM [20] can be considered as a master program that interfaces QGRAF with MINCER 24] and MATAD 23]. DIANA [59] is another Feynman diagram analyser with graphics facilities that exploits the power of FORM and is based on QGRAF for the generation of the Feynman diagrams. However it does not include, for applications to loop calculations, all the ingredients we listed in the diagram generators of the complete automatic system since it lacks the module for loop integration. For a full review of such packages, see [20].

The new code aITALC 60] on the other hand, could be considered as a fully automated code for the calculation of cross sections. Built on DIANA, it goes a step further by adding the LOOPTOOLS 41] library. The code is restricted to the evaluation of $2 \rightarrow 2$ processes at one-loop with only external fermions. It does not include hard bremmstrahlung.

In a different context, MicrOMEGAs 61] is a code written in $\mathrm{C}$ for the automatic evaluation of the relic density of dark matter in supersymmetry. By default the lightest neutralino is assumed to be the lightest supersymmetric particle, but the user can set any supersymmetric particle (not including Higgses) to be the LSP. The code is built on CalcHEP 62 which generates, while running, the subprocesses needed for a given set of $\mathcal{M S S M}$ parameters ${ }^{4}$.

\footnotetext{
${ }^{4} \mathrm{CalcHEP}[62$ is an outgrowth of CompHEP [26].
} 


\subsection{Implementation of the model file}

The model file contains all the information about the theoretical model where the calculation is to be performed. In particular the whole set of particles of the model and their interactions must be transcribed in a machine readable format. To perform the calculation one then needs to define the particles and write down the Feynman rules in terms of all possible vertices needed to build up the Feynman graphs. For the electroweak theory this means, among other things, writing all the set of Feynman rules as listed in Appendix B. At the loop order, the definition requires that one specifies counterterms after having set the renormalisation procedure. This means for instance that the loop order of a vertex be also specified. The set of one-loop vertices that need to be generated at one-loop in the electroweak theory is listed in Appendix $\mathbf{F}$

Figure 2: An example of a model file in GRACE.

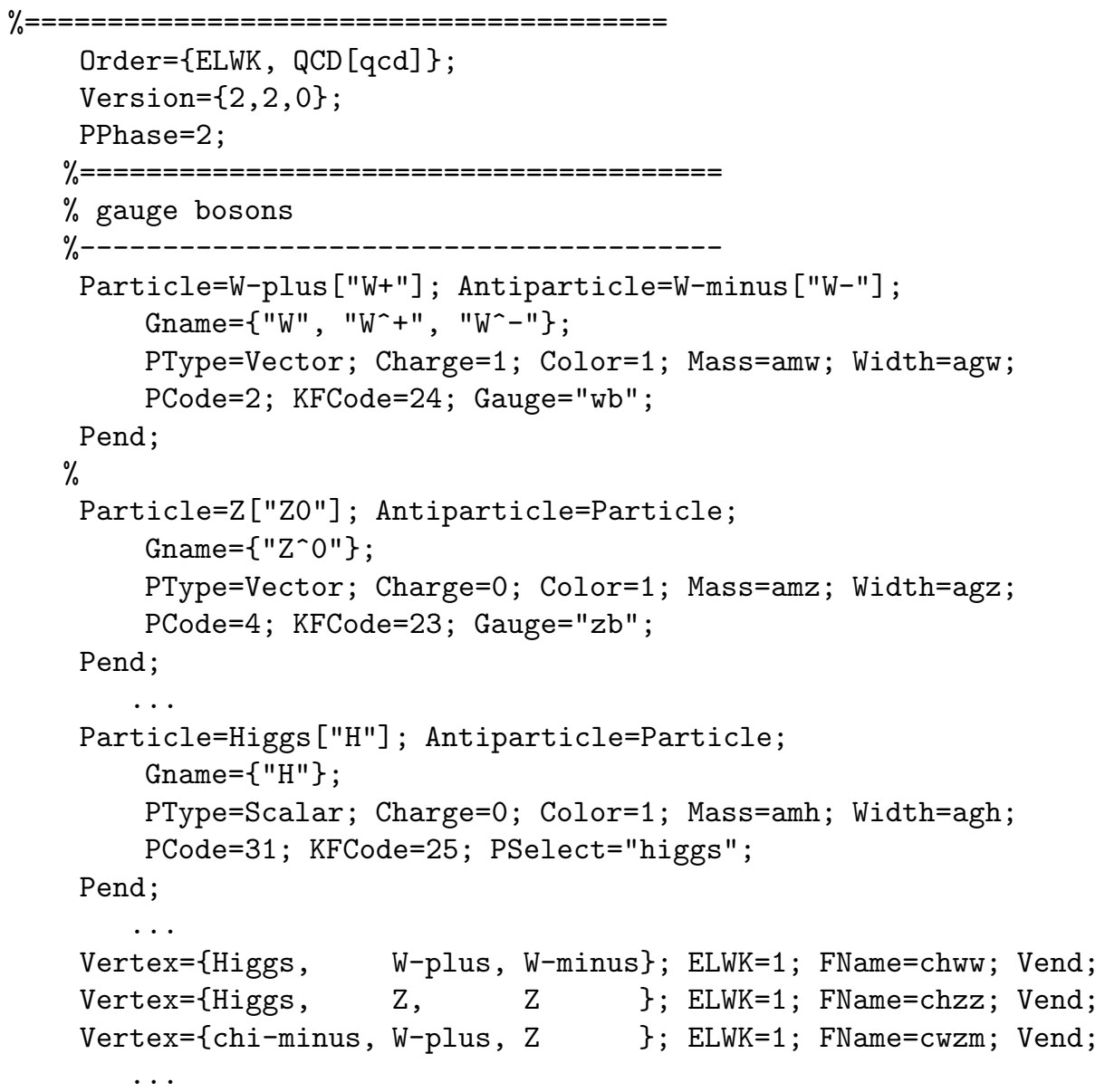

Most automatic systems read model files in which the information on the particles and the vertices, through the Feynman rules, are coded manually. Figure 2 shows an example of a model file for the GRACE system. At first some options are specified. In this example, the name 
of the coupling constants are defined by Order=.... Each particle is described through a set of properties such as the name of the particle, spin, electric charge, representation of internal symmetries, whether it is massive or massless and so on. The interactions of the particles are defined by a set of vertices. This example shows the definition of some scalar-vector-vector, SVV, vertices which consist of the list of interacting particles, the order of the coupling constants and the name of the coupling constants used in the generated code. For a model such as the $\mathcal{S M}$ or any renormalisable model, all types of SVV vertices have a common Lorentz structure. As with other types of vertices FFV,VVV etc.., see Appendix B, the Lorentz structure is exploited in building up the Feynman amplitude, as will be shown below.

Although it is easy to code a model file by hand for a simple model such as QED, it is not always an easy task for a more complicated model. For example, the $\mathcal{M S S M}$ consists of more than 80 particles resulting, at tree-level alone, in more than 3,000 vertices. This is the reason why it is more desirable to construct model files automatically from a Lagrangian, with a minimum of human intervention. In this case the set of Feynman rules and vertices is generated automatically. Using dedicated programming languages, software packages have been developed for the automatic generation of Feynman rules. One can cite beside LanHEP 63. originally designed to work with CompHEP, the codes included in FDC 33] or gss 64. In this case, a Lagrangian is usually given in a quite compact and symmetric form. Gauge fixing terms and ghost terms are added to it. The latter can even be implemented automatically by first defining the symmetries of the theory. For instance, in LanHEP, using the BRST transformation 65, 66. (see Appendix A) as done in section 5.2 one can make the system automatically derive the ghost Lagrangian and the corresponding Feynman rules. In the following we sketch some of the steps in automatically deriving the Feynman rules from an algebraic implementation of the Lagrangian and by applying some simple set of rules. We take LanHEP as an example without going into the details of the procedure, the interested reader should consult the manual of LanHEP 63 .

One first needs to define the particles of the model as shown in Fig. 3 for the bosons of the electroweak model.

Figure 3: Particle description in LanHEP

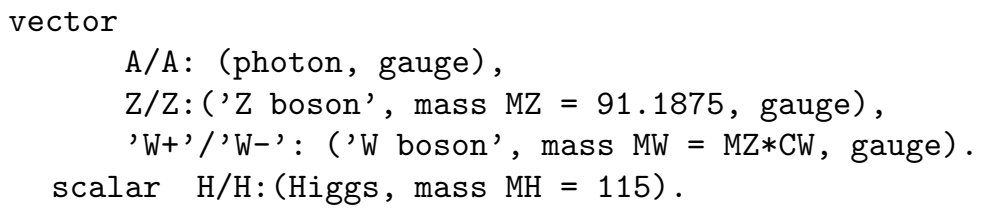

Figure 4 shows how parts of the Higgs Lagrangian are entered once the Higgs doublet pp (.f refers to the Goldstones), and the covariant derivative $\mathrm{Dpp}^{`} \mathrm{mu}^{\wedge} \mathrm{a}$ are defined. The command Iterm specifies a term in the Lagrangian. Shifts to introduce the wave function renormalisation are performed through the command transform, see Fig. [5. It is also possible in LanHEP to introduce a command brst for the BRST transformations [65, 66]. In the example of Fig. 6. by acting on the gauge-fixing function, this generates the ghost Lagrangian. 
Figure 4: Entering the Higgs interaction in LanHEP

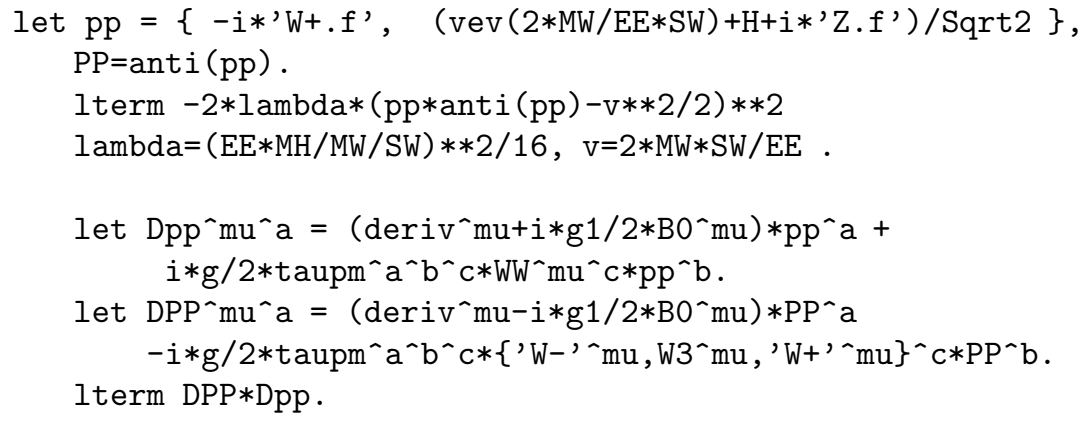

Figure 5: Introducing wave function counterterms in LanHEP

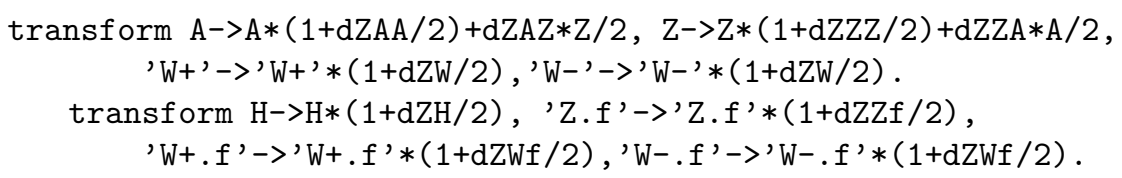

Figure 6: Introducing gauge-fixing and ghosts in LanHEP

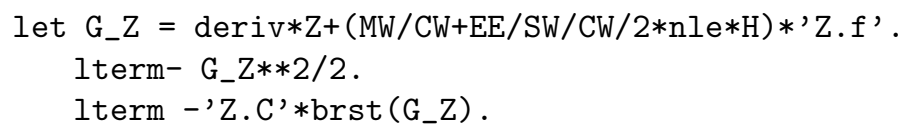

\subsection{Feynman Diagram Generation}

The automatic generation of Feynman diagrams necessary for the computation of a process within a model proceeds after the user has defined the process through an input file. Fig[7]shows an example of an input file specifying a process. In this example it is $e^{+} e^{-} \rightarrow W^{+} W^{-} \gamma$ at treelevel. The input data specifies a theoretical model (here sm.mdl), the order of perturbation by counting the power in the coupling constants (here ELWK=3) and by choosing the initial $\left(e^{+}, e^{-}\right)$ and final particles $\left(\gamma, W^{+}, W^{-}\right)$. In the GRACE input file, Kinem="2302" identifies a choice of kinematics in the kinematics library to be used for the integrated cross section ${ }^{5}$.

A typical algorithm for the generation of the Feynman diagrams will be the following:

1. Generate the number of vertices.

The number of vertices is restricted by the order of the coupling constants for the physical process and is given by the input file, see Fig[7. Each vertex has a fixed number of propagators and external particles to be connected.

\footnotetext{
${ }^{5}$ For details about the format of the input file, see Ref [34].
} 
Figure 7: An example of the input file in the electroweak $\mathcal{S} \mathcal{M}$ for the scattering process $e^{+} e^{-} \rightarrow W^{+} W^{-} \gamma$ at tree-level.

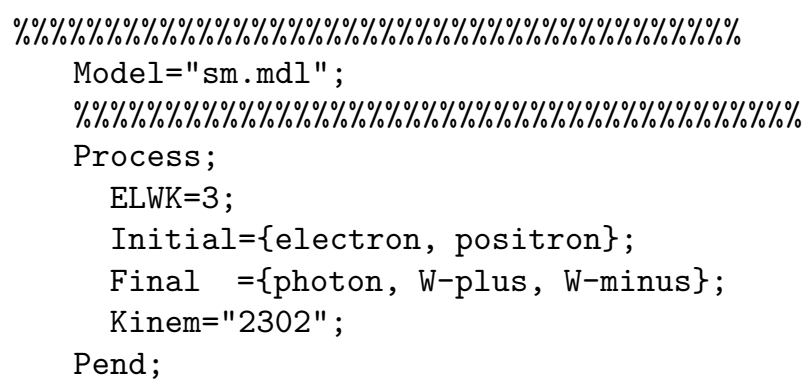

2. Connect vertices with propagators or external particles.

There are multiple ways to connect vertices. All possible configurations are to be generated.

3. Particle assignment.

Particles are assigned to propagators confirming that the connected vertex is defined in the model. As there will be many ways to assign particles to propagators, all possible configurations are to be generated. In generating the Feynman diagrams, conservation laws such as electric charge and fermion number conservation will be employed in order to avoid fruitless trials.

The generation of Feynman diagrams borrows heavily from graph theory. In the following let us call a vertex or an external particle a node. Similarly, let an edge be a connection between two nodes, which may be a propagator or a connection between a vertex and an external particle. Thus an edge is expressed by a pair of two nodes (which are connected by the edge). The diagram or graph generation process is to construct edges in all possible ways.

We show an example of an output file generated by the GRACE system in Fig. 8 for the process we defined in the input file of Fig. [7 namely $e^{+} e^{-} \rightarrow W^{+} W^{-} \gamma$. The first part of this file describes the information about the physical process. The first generated diagram, Graph=1, consists of the 5 external particles and the 3 vertices. To this corresponds 7 edges that connect these nodes; for example, the final photon (labelled as node 2 and 3rd external particle) is connected to the $\gamma W^{+} W^{-}$vertex (node 7) by edge 3. Particles are defined as incoming to the node. This information is used by another program such as the code for drawing diagrams. This reconstructs the structure of diagrams, places nodes on a graphic device and connects them by edges as shown in Fig 9 .

It is to be noted that although vertices of the same kind are not distinguished from each other, for instance in our example the vertex $W^{+} W^{-} \gamma$ appears twice in Graph 1 (see Fig. 9), they will be distinguished in a program, usually through a sequence of numbered labels, in our case node 6 and 7 (see Fig. 8). On the other hand, since a Feynman graph is a topological object, it is independent of the way one assigns the sequence of numbers to nodes. Such simple algorithms will produce diagrams, which are topologically the same, many times. The problem 
Figure 8: An example of information about a generated diagram in GRACE. The first part below defines the external particles. It is followed by defining the connections between the nodes and the vertices in the form "node number $=\{$ edge numbers $\}$ ".

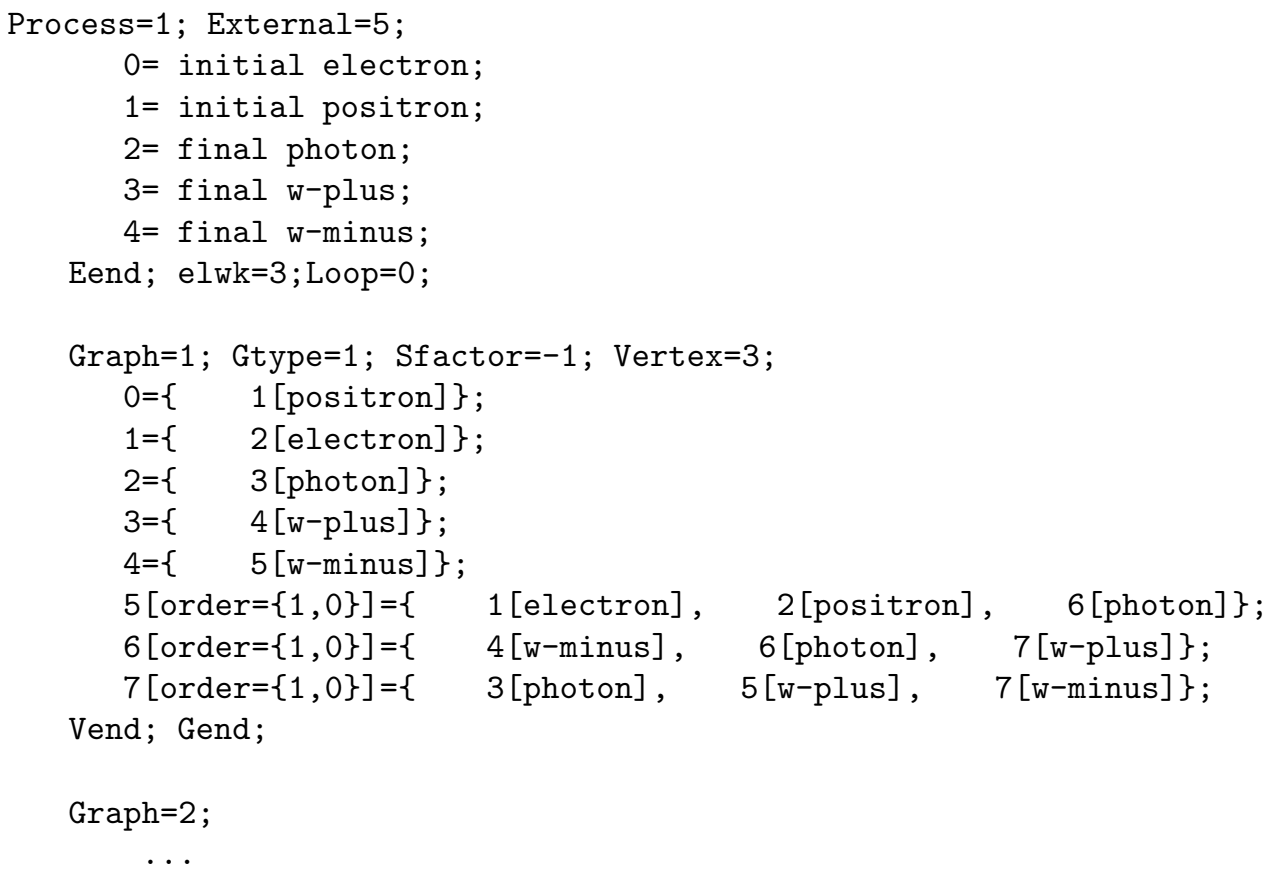

of diagram generation is not so much to generate diagrams but to avoid such duplications. Although it is not so difficult to avoid generation of duplicated diagrams when limited to special cases such as QED [18] or tree processes, the problem of redundancy gets exasperated when loop diagrams are generated.

A general method of graph generation avoiding duplicated graphs, applicable to any process with any order in the coupling constants, has been developed by graph theorists [67]. With this method, one can calculate the symmetry factors of the Feynman diagrams at the same time. Unfortunately, such a method is not efficient enough for a large number of external particles or loops 68. With some optimization, this method was first applied to the generation of Feynman diagrams in the code QGRAF 21. Another technique of optimisation and acceleration was proposed in 69. The GRACE system exploits the latter algorithm and acceleration for the generation of Feynman diagrams. Appendix D presents in some detail the issue of graph duplication and calculation of the symmetry factors and the need for optimising and accelerating the generation of Feynman diagrams. Examples beyond one-loop and outside the electroweak model are given to illustrate these issues. 
Figure 9: An example of a diagram drawn by GRACE based on the file in Fig. 8. Here each dot is a node. In this figure we have only labelled those nodes that correspond to the external particles according to the listing in Fig. 8. The numbers in parentheses correspond to the edges as defined in Fig. 8 also.

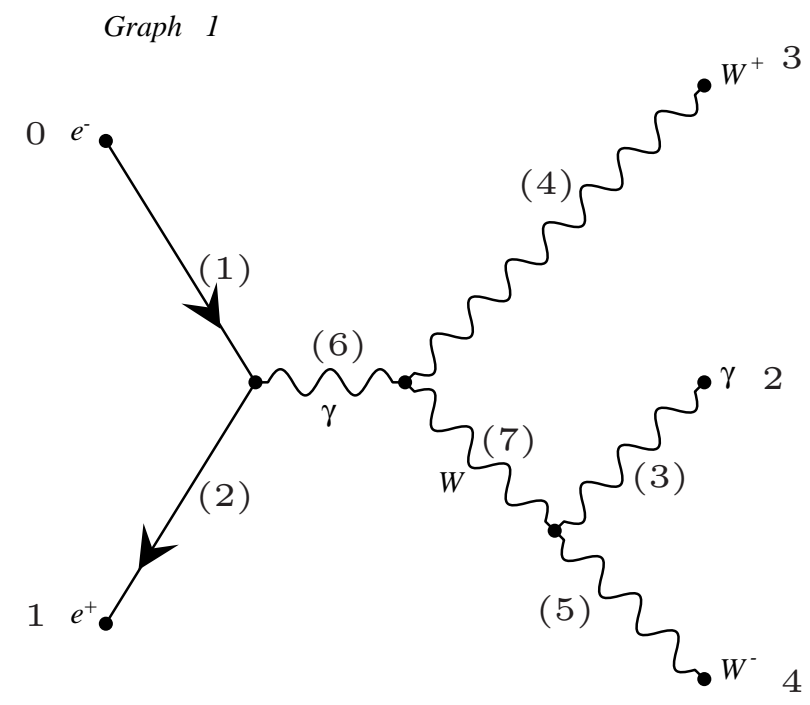

produced by GRACEFIG

\section{Automatic systems at tree-level}

We first start by giving the main lines of how the automatic code builds up and calculates Feynman amplitudes from the Feynman graphs at tree-level. It is very much a transcription of a calculation by hand.

\subsection{Squared matrix elements}

One method is to calculate squared amplitudes. Here projection operators, Dirac's gamma matrices and the sum over the polarizations of vector particles can be handled symbolically. This symbolic treatment of the mathematical expression is performed with a symbolic manipulating system such as REDUCE or FORM[12] in the case of GRACE or a specially developed package in the case of CompHEP 26]. Symbolic calculation in FeynCalc/FormCalc 29] uses MATHEMATICA and FORM. An automatic system generates a source code for a symbolic manipulating system in accordance with the Feynman rules of the model. As the number of particles increases, the number of Feynman graphs grows very rapidly. This means that this method is not suitable in these cases as it requires computing the square of the number of Feynman diagrams. Moreover this method is clearly unsuitable if one requires information on the polarisations. 


\subsection{Spinor technique: a worked out example in GRACE}

Another method employs the spinor technique. Spinors and gamma matrices are dealt with in a numerical way. A library of all possible types of vertices and propagators are defined as subroutines to be called for a numerical calculation. The CHANEL [57] library for GRACE [25] and HELAS [70] library for MadGraph [32] are examples of such subroutines. The automatic system calls these libraries according to the structure of the Feynman graphs. Output of the system is a complete code for the numerical calculation of differential cross sections without calling any other package. Since this method calculates helicity amplitudes directly, it is natural to calculate polarized cross sections. The necessary CPU time is proportional to the number of Feynman graphs in this method.

We will here show in some detail how the helicity amplitude method can be implemented in an automatic code such as GRACE. The method is purely numerical. The amplitude corresponding to each Feynman graph is first decomposed into vertex sub-amplitudes. Each of these sub-amplitudes is read from a pre-defined model file library so that one only has to call the library, in this case CHANEL, where these sub-amplitudes are defined. In order to achieve this, the propagators that appear as internal lines are expressed as a product of wave functions.

Figure 10: A Feynman graph contributing to $e^{+} e^{-} \rightarrow W^{+} W^{-} \gamma$.

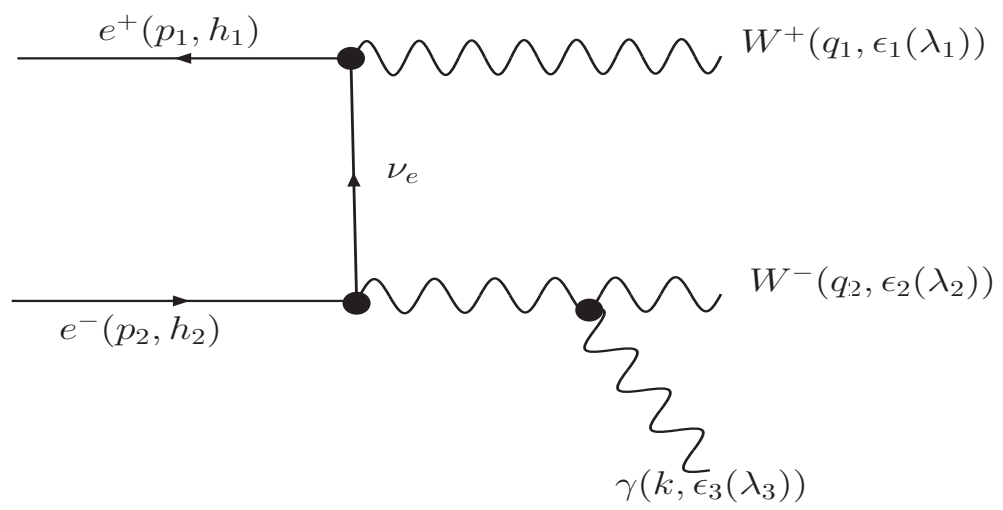

Consider the scattering amplitude corresponding to the Feynman graph shown in Fig. 10 as an example. $p_{1}, p_{2}, q_{1} q_{2}$ and $k$ are momenta of $e^{+}, e^{-}, W^{+}, W^{-}$and $\gamma$, and $h_{1}$ and $h_{2}$ are helicities of $e^{+}$and $e^{-}$, and $\epsilon_{1}\left(q_{1}, \lambda_{1}\right), \epsilon_{2}\left(q_{2}, \lambda_{2}\right)$ and $\epsilon_{3}\left(k, \lambda_{3}\right)$ are polarization vectors of $W^{+}, W^{-}$and $\gamma$, respectively with the corresponding helicity $\lambda_{1,2,3}$.

The scattering amplitude for this contribution is given by

$$
\begin{aligned}
T_{f i}= & \bar{v}\left(p_{1}, h_{1}\right) c_{e W}^{\eta} \epsilon_{1 \eta}\left(q_{1}\right) S_{F}\left(-p_{1}+q_{1}\right) c_{e W}^{\mu} u\left(p_{2}, h_{2}\right) \\
& \times D_{F \mu \nu}\left(q_{2}+k\right) c_{W W \sigma}^{\nu \rho \sigma}\left(q_{2}+k,-q_{2},-k\right) \epsilon_{2 \rho}\left(q_{2}\right) \epsilon_{3 \sigma}(k),
\end{aligned}
$$

where $c_{e W}^{\eta}$ and $c_{W W \gamma}^{\nu \rho \sigma}$ express electron- $W$ and photon- $W$ couplings, respectively, and they are 
given by:

$$
c_{e W}^{\eta}=\frac{e M_{Z}}{\sqrt{2\left(M_{Z}^{2}-M_{W}^{2}\right)}} \gamma^{\eta} \frac{1-\gamma_{5}}{2}
$$

and

$$
c_{W W \gamma}^{\nu \rho \sigma}(p, q, r)=e\left[(p-q)^{\sigma} g^{\nu \rho}+(q-r)^{\nu} g^{\rho \sigma}+(r-p)^{\rho} g^{\sigma \nu}\right] .
$$

In CHANEL, propagators are expressed as a bi-linear form of wave functions:

$$
S_{F}(p)=\frac{\sum_{\alpha i} w_{\alpha, i} U^{\alpha}\left(h^{(i)}, p^{(i)}\right) \bar{U}^{\alpha}\left(h^{(i)}, p^{(i)}\right)}{p^{2}-m^{2}}
$$

and

$$
D_{F \mu \nu}(p)=\frac{\sum_{i} w_{i} \epsilon_{\mu}^{(i)}(p) \epsilon_{\nu}^{(i)}(p)}{p^{2}-m^{2}},
$$

where $w_{i, j}$ and $w_{i}$ are c-numbers and weight factors for the decomposition of propagator. $U^{\alpha}$ represents either a spinor $u$ or $v$ depending on the value of index $\alpha$. Momenta $p^{(i)}$ are calculated from the (off-shell) fermion momentum $p$.

The amplitude then writes as a product of vertex sub-amplitudes

$$
\begin{aligned}
T_{f i}= & D\left(-p_{1}+q_{1}, 0\right) D\left(q_{2}+k, m_{W}\right) \sum_{\alpha, i} w_{\alpha, i} \sum_{l} w_{l} \\
& \times V_{e W^{+}}^{(\alpha, i)} V_{e W^{-}}^{(\alpha, i, l)} V_{W W \gamma}^{(l)},
\end{aligned}
$$

where

$$
\begin{aligned}
D(p, m) & =\frac{1}{p^{2}-m^{2}}, \\
V_{e W^{+}}^{(\alpha, i)} & =\bar{v}\left(p_{1}, h_{1}\right) c_{e W}^{\eta} \epsilon_{1 \eta}\left(q_{1}\right) U^{\alpha}\left(\left(-p_{1}+q_{1}\right)^{(i)}, h^{(i)}\right), \\
V_{e W^{-}}^{(\alpha, i, l)} & =\bar{U}^{\alpha}\left(p^{(i)}, h^{(i)}\right) c_{e W}^{\mu} \epsilon_{\mu}^{(l)}\left(q_{2}+k\right) u\left(p_{2}, h_{2}\right),
\end{aligned}
$$

and

$$
V_{W W \gamma}^{(l)}=c_{W W \gamma}^{\nu \rho \sigma}\left(q_{2}+k,-q_{2},-k\right) \epsilon_{\nu}^{(l)}\left(q_{2}+k\right) \epsilon_{2 \rho}\left(q_{2}\right) \epsilon_{3 \sigma}(k) .
$$

Further details of how the spinors and polarisation vectors are represented, together with the weight factors can be found in 25. The fermion-fermion-vector (FFV) of the $\mathcal{S} \mathcal{M}$ vertex parts $V_{e W^{+}}^{(\alpha, i)}$ and $V_{e W^{-}}^{(\alpha, i)}$ are calculated with the help of the CHANEL subroutine SMFFV while the 3 -vector (VVV) vertex part $V_{W W \gamma}^{(l)}$ by the subroutine SMVVV. These subroutines calculate all combinations of helicity states and of spinors $u$ and $v$. Therefore given a set of momenta and helicities, these vertex parts return a number automatically.

Summation over indices pertaining to the fermion propagators is made through a call to the subroutine SMCONF:

$$
V_{e e W^{+} W^{-}}^{(l)}=\sum_{\alpha, i} w_{\alpha, i} V_{e W^{+}}^{(\alpha, i)} V_{e W^{-}}^{(\alpha, i, l)}
$$


The last free index $l$, which corresponds to the propagator of a vector particle is through a call to the subroutine SMCONV:

$$
\sum_{l} w_{l} V_{e e W^{+} W^{-}}^{(l)} V_{W W \gamma^{(l)}}^{(l)}
$$

The program generates a sequence of subroutine calls in the following order:

1) For a given process, subroutines SMEXTF and SMEXTV are called first, encoding information on the external fermions (SMEXTF) and external bosons (SMEXTV) in a form suitable for the calculation of the vertices. Since this part is common to all graphs, it is generated once before calculating an amplitude of the first graph.

2) Momenta of propagators are defined by taking linear combinations of external momenta based on momentum conservation. Then the denominators of the propagators are calculated by the subroutine SNPRPD.

3) The decomposition of the numerator of the propagators as a bi-linear product of wave functions is carried through SMINTF for fermions and SMINTV for vectors.

4) The different subroutines such as SMFFV or SMVVV are called for calculating vertices subamplitudes.

5) These vertices are connected in correspondence with the propagators of the graph, which is realized by appropriate subroutines such as SMCONF and SMCONV.

6) Before the amplitudes are summed over all graphs a reordering of the particles, so that they appear in the same sequence for all the graphs, is carried through a special routine.

7) After summing over all diagrams, the helicity amplitudes are squared. Summation over spin states can also be performed automatically by a call to a dedicated routine.

The generated code for this example is shown in Fig. 11. A more detailed description of the CHANEL library is given in 25].

\subsection{Dealing with QCD and supersymmetry}

Extension of both the squared matrix elements and spinor techniques to supersymmetric models requires a proper treatment of Majorana particles. Calculation with Majorana particles involves the charge conjugation operator. When a symbolic manipulation system is employed, the symbolic treatment of this operator must be implemented. A simple algorithm[71] has been constructed where this operator is confined to the conjugated vertices, so in effect we only deal with the usual Dirac propagator while the vertices do not explicitly invoke charge conjugation. In the case of the spinor technique, we only need to add new appropriate subroutines to a library, such as CHANEL.

For QCD, the automatic calculation refers to the calculation of the partonic hard scattering part of the matrix elements. The colour factor of a graph is separated out. However, Feynman 
Figure 11: The code for the helicity amplitude corresponding to the graph in Fig. 10.

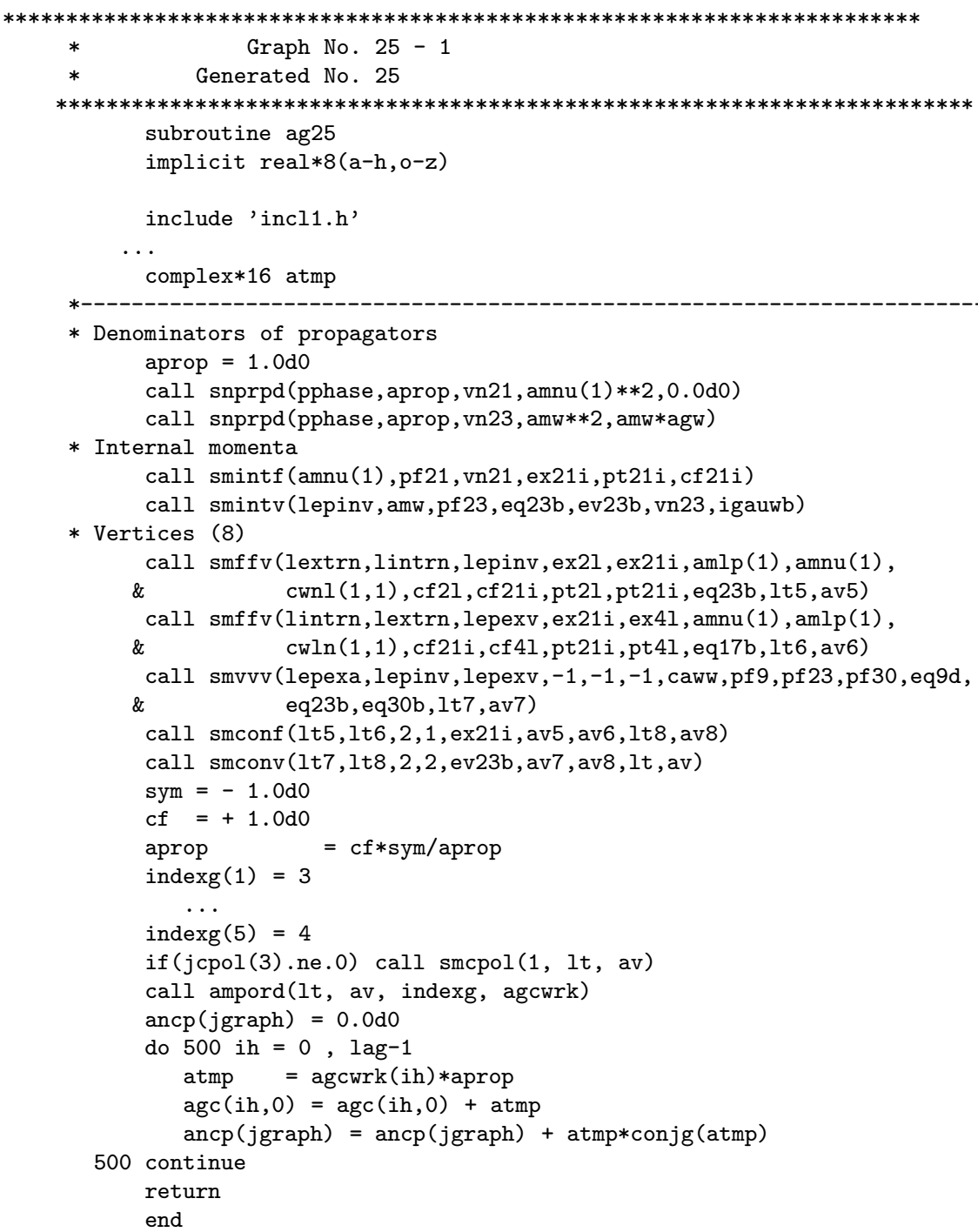


rules for the four point vertex of the gluon is expressed in a mixed form of colour matrices, through the $S U(3)$ structure constants, $f^{a b c}$ and Lorentz parts:

$$
\begin{gathered}
g_{s}^{2}\left[\left(f^{a c e} f^{b d e}-f^{a d e} f^{c b e}\right) g_{\alpha \beta} g_{\gamma \delta}+\left(f^{a b e} f^{c d e}-f^{a d e} f^{b c e}\right) g_{\alpha \gamma} g_{\beta \delta}\right. \\
\left.+\left(f^{a c e} f^{b d e}-f^{a b e} f^{c d e}\right) g_{\alpha \delta} g_{\gamma \beta}\right]
\end{gathered}
$$

There are two ways for the separation of these factors. One is that the Feynman graph is generated with the original quartic vertex but the amplitude generator expands this vertex into a sum of three terms where the colour index is factored out. The other method, also used in CompHEP for example, introduces an auxiliary field which interacts through a vertex with two gluons. The Feynman graph generator constructs the quartic gluon vertex as the sum of graphs consisting of $s, t$ and $u$ type exchanges of the auxiliary field. The auxiliary field method is also best suited in the case of the $\mathcal{M S S M}$ where additional coloured particles with 4-point vertices exhibiting a variety of colour structures are needed.

\subsection{Checking the result at tree-level}

The $\mathcal{S M}$ and the $\mathcal{M S S M}$ are gauge theories. One can exploit the gauge freedom to check the result of the calculation of a cross section or helicity amplitude. For the calculation based on the spinor technique, it is easy to keep the gauge parameters as variables in the generated code without increasing the $\mathrm{CPU}$ time. For the squared matrix technique that relies on symbolic manipulation, checks that are based on varying the usual 't Hooft-Feynman parameter lead to very complicated and very large expressions. Checks are much more manageable with generalised non-linear gauges that will be discussed in detail later in Section 5 .

\subsection{Automatic tree calculations without Feynman graphs}

Let us briefly mention that there exists codes for the automatic calculation of amplitudes that do not require the standard textbook approach based on Feynman graphs. ALPHA [35] uses an algorithm based on an iterative solution of the equation of motion whereas HELAC [36 is based on solving the Schwinger-Dyson equation. An acceleration method is developed in [37] and is applicable also to the methods using Feynman amplitudes. All these methods exploits a binary representation of the momenta involved in the tree-level amplitude calculation.

Let $p_{i}, i=1, \ldots, N$ be the momenta of the external particles for a certain amplitude. Momentum conservation imposes the restriction $\sum_{i} p_{i}=0$. A momentum $q$ of a propagator in a tree graph is expressed as $q=\sum_{i} a_{i} p_{i}$, where $a_{i}$ is either 0 or 1 . Thus the possible number of momenta appearing in the calculation is bounded by $2^{N-1}-1$ and only a finite limited number of vertices appear in the calculation. This helps to construct tree $N$-point Green's function combining these vertices in a kind of matrix operation.

\subsection{Kinematics and event generation}

Having obtained the amplitudes or the squared matrix elements, the last step is the integration over phase space to obtain the cross section. One may also want to generate events for the 
analysis of experimental data. For multi-particle final states, phase space integration means integrating over many variables. Since the amplitudes themselves, the integrand, are most often lengthy and have a complicated structure in these variables, Monte Carlo integration packages such as VEGAS [72] and BASES [17] are used. Even if the integrand were a smooth function over the whole phase space without narrow peaks, naive use of a numerical integration package may cause problems as it would be difficult to reach the required accuracy especially for a large multidimensional space or when one deals with complicated boundary configurations. Narrow peaks can occur for example where the momentum of an internal particle is such that it approaches its mass-shell. The amplitude could blow up. As these peaks give an important contribution to the results, it is necessary to catch the detailed structure of these singularities and to integrate around these peaks within good accuracy. In this case the Monte Carlo integration package needs to accumulate enough sampling point around these peaks. When singularities run parallel to integration axes, VEGAS or BASES optimizes the distribution of sampling points looking at the projected values on the axes.

It is fair to say that it is this stage of the automatic calculation of cross sections in high energy physics which requires most intervention by the user. This is due to the fact that there is at the moment no general purpose integrator that can automatically catch the different types of singularities and suitably adapt itself to properly treat all the singularities present in a process with enough accuracy. Several integration packages have been developed [73, 74, 75] for handling many singular situations but none is general enough that it can be trusted to run independently and give an accurate result in any situation. The user should select good integration variables so that the singularities are arranged along the integration variables. This selection of the integration variables depends, of course, on the process. Some systems, such as GRACE , include a library of kinematics suitable to handle a number of topologies for the singularities for a given number of external legs.

In the case of QCD, the partonic cross sections and amplitudes need, for example, to be convoluted with parton distribution functions. The code can also provide packages for parton shower and hadronisation.

Once a numerical code of a differential cross section is obtained, one can generate unweighted events by a program package such as SPRING [17] using the result of the integration calculated by BASES. Unweighted simulated events, generated in accordance with the theoretical predictions are very useful tools to analyse the experimental data and take into account the complex structure of the detectors.

\section{Automatic systems for one-loop processes}

Extension of an automatic system from tree-level to one-loop processes is, in principle, straightforward because all the ingredients needed for one-loop calculations in a renormalisable theory are known. In practice, however, quite a few of the new features not met when handling processes at tree-level pose a real challenge and are fraught with technical difficulties. This explains why although there has been a proliferation of automatic systems for tree processes since the beginning of the 1990s, only a couple can tackle one-loop processes. Currently two systems are available, GRACE-loop and FeynArt/FeynCalc/FormCalc 27, 29, 30, 31] which apply both to 
the electroweak sector of the $\mathcal{S M}$ and the $\mathcal{M S S M}$. Application to QCD at one-loop is at the stage of development by some groups but a general purpose code is still not completed yet. In this section we describe what kind of problems one encounters in the construction of an automatic system at one-loop based on the experience we gained while developing GRACE-1oop for electroweak processes in $e^{+} e^{-}$colliders.

An automatic system for one-loop should be composed of the following ingredients:

1. Proper graph generator. This should provide all one-loop diagrams for a given process. Most graph generators that work for tree-level handle at least one-loop diagrams. We have already described the general algorithm of graph generation in Section 2.3 .

2. Amplitude generator. This very much depends on how to calculate one-loop amplitudes. Both the helicity amplitude formulation and/or the squared matrix elements technique are possible as explained later.

3. The model file should include a library for counterterms. This should contain all terms necessary to compensates ultraviolet divergences. For a given theoretical model, one can have different libraries reflecting the fact that one can choose between different renormalisation and regularisation schemes. In GRACE, ultraviolet(UV) divergences are regularised through dimensional regularisation and the electroweak sector is renormalised on-shell, see section 6.1 Note in passing that there should also be a prescription for handling infrared(IR) divergences. In GRACE this is done by giving the photon an infinitesimal mass, see section 8.1.2. These libraries have a structure similar to those defined for the tree-level model files.

4. A library for one-loop integrals. This should include analytic formulas for 2-, 3- and 4point scalar integrals, see section [7.1] 5 - and 6-point functions can be expressed by a sum of 4-point functions, section 7.6. As we will see in section 7.1, a scalar integral is an integral over the loop momentum where the integrand is a product of the denominators of the various propagators inside the loop, so that no loop momentum appears in the numerator of the integrand. When products of loop momenta are involved one speaks of higher rank tensors.

5. Beside the aforementioned reduction of 5- or 6-point diagrams to 4-points, one needs another library which decomposes higher rank tensors of a box diagram to relevant scalar integrals and surface integrals, namely 3-point functions. The reduction formulas for higher rank tensors of vertex diagrams is needed as well. There are a few algorithms dealing with the last two points. One needs very efficient algorithms and numerical routines here, since this steps can take up a large fraction of the CPU time. This is developed further in this article in section 7.3

6. Ways to check the results. This is essential and must be performed. We consider it as the most important part of the system because the calculation is much more complicated than the tree processes. Lacking these tests one cannot be convinced that the obtained results are correct. Usually, one checks UV and IR finiteness. Gauge invariance is also a very powerful tool, however the familiar 't Hooft gauges are not suitable for multi-leg 
one-loop processes. GRACE incorporates a series of parameters derived from a generalised non-linear gauge.

The theoretical background and the algorithms necessary for the construction of the libraries related to the points 3,4,5 above will be developed in detail in sections 8.1.1, 8.1.2, 7.1, 7.6] and 7.3 respectively. The gauge-parameter independence check in the last point above is crucial and could be considered as pivotal. Beside being a most powerful check on the calculation it also has a bearing on how the libraries for the reduction of the one-loop integrals are constructed. The choice of special gauges here is almost mandatory. This is the reason we pay so much attention to the implementation of the non-linear gauge in this article. Although we will, in the next sessions, get back to the details of all the points listed above and before we point to some features related to the technical implementation of the one-loop radiative corrections in the electroweak part of the $\mathcal{S M}$ in the automatic code GRACE, it is worth to briefly expose the gauge-parameter check.

\subsection{Gauge parameter independence}

The most stringent test for the consistency of the calculation will be provided by the gaugeparameter independence of the cross section. In tree-level calculations with an automatic code any gauge fixing will do. This is because the inclusion of any modifications brought by the gauge fixing Lagrangian and associated ghost Lagrangian is always easy since no loop integration is needed. Typically, the standard $R_{\xi}$ gauge fixing is used where the gauge parameter hides in the propagators of the gauge particles $\gamma, Z, W^{ \pm}$, and unphysical scalars of the theory. For example in a general $R_{\xi}$ gauge, the $Z$ propagator writes

$$
D_{Z}^{\mu \nu}\left(q^{2}\right)=\frac{1}{q^{2}-M_{Z}^{2}+i \epsilon}\left[g^{\mu \nu}-\left(1-\xi_{Z}\right) \frac{q^{\mu} q^{\nu}}{q^{2}-\xi_{Z} M_{Z}^{2}+i \epsilon}\right] .
$$

This formulation of gauge fixing, however, is not well suited when loop calculations are involved. The "longitudinal" part, in the second term of the propagator, constitutes an obstacle in carrying out the loop integration, due to the appearance of not only unphysical thresholds, $\xi_{Z} M_{Z}^{2}$, functions but also the introduction of higher rank tensors, $q^{\mu} q^{\nu}$, and higher order $N$ point functions since this part involves "two" denominators. Since as we will see, the reduction of tensor loop integrals and higher $N$-point functions is very much time consuming it is desirable to find another formalism for gauge fixing which allows the propagators to take as simple form as possible, $\xi_{Z}=1$. This can in fact be realized in the non-linear-gauge(NLG) fixing formulation [54. With this kind of gauge fixing, the propagators can be taken to correspond to $\xi_{i}=1$ for all gauge particles but additional gauge parameters, through derivatives in the gauge-fixing, modify a large number of vertices where the gauge and unphysical (Goldstones and ghosts) are involved. We will take a generalised gauge with five gauge parameters in total, $\tilde{\alpha}, \tilde{\beta}, \tilde{\delta}, \tilde{\varepsilon}, \tilde{\kappa}$. Although this means some more work in redefining the model file as compared to the linear $R_{\xi}$ gauge which only modifies a few propagators and also because renormalisation is slightly more involved than in the by-now textbook linear $R_{\xi}=1$ gauge, implementation of the non-linear gauge is worth the investment if one is interested in a general purpose automatic loop calculator. One then has the possibility of performing gauge-parameter independence checks, 
involving the 5 parameters, on a large scale calculation. We will see in section 8.2 that, though each diagram out of a set of some hundreds diagrams shows a dependence in a gauge parameter, summing over all diagrams gives a gauge independent result. This is a very powerful check on the calculation and on the automatic system.

\subsection{Generation of matrix elements}

As is in the case of tree processes, in general the matrix elements can be obtained either through the helicity formalism or one can obtain directly the squared matrix elements with the help of some symbolic manipulation programs. GRACE-loop has both versions.

The procedure to get the helicity amplitudes is as follows. Once a diagram is generated a REDUCE code is produced which contains an expression for the corresponding amplitude. This is just a "reading of the amplitude" in REDUCE. We do not take any trace of $\gamma$ matrices unless a fermion loop is formed inside the diagram. The role of REDUCE is only to rearrange the generated terms. First, the Feynman parameters for the loop integration are introduced, see Section $\mathbf{7}$ and Eq. 7.2. The loop momentum $l$, see Eq. 7.1] is shifted so that one has denominators containing $l$ only through $l^{2}$. The amplitude now contains not only the loop, external momenta and Feynman parametric variables but also strings of $\gamma$ matrices.

Operations are done in $n$-dimension. All the Lorentz contractions are taken in $n$-dimension if the pair of indices does not bridge two fermion lines. Then, without taking the trace, every product of $\gamma$ matrices along the fermion lines is replaced by a corresponding symbol which works as a function when the code is converted to a FORTRAN source code. The remaining contractions and the calculation of the products of $\gamma$ matrices are left to be done numerically in particular by the CHANEL library that we already described in the case of tree-level amplitudes. As stated, REDUCE is used, in this approach, to get the final form of the amplitude, but only for the rearrangement of terms and the functions such as spur (Trace) and index (contraction) are applied only to quite a limited part of the manipulation.

Now the obtained amplitude can be regarded as a polynomial with respect to the loop integration parameters. Each monomial is replaced by a symbol which represents a one-loop integral with the numerator corresponding to that monomial. Thus the amplitude can be calculated when a library of loop integrals is supplied, see Section 7 . Since we do substantially nothing with the amplitudes, it is quite easy to get them.

On the other hand, in the second approach traces of all $\gamma$ matrices and contractions of indices are applied systematically on the product of a tree amplitude with a loop amplitude. Again dimensional regularisation is assumed in the case of UV divergent diagrams. The coefficient of a monomial of the parameters for the loop integration is merely a polynomial composed of external momenta. That monomial is also replaced by a symbol which is a substitute for the corresponding loop integral. This approach generates much larger expressions than the helicity approach, see Table 1. It may happen that REDUCE is not able to complete all the manipulations because too huge intermediate expressions are produced.

The helicity amplitude formalism is certainly desirable because it can provide the spin information. However, the sums on spin states required for external and internal particles produce a certain number of arithmetic operations. This would not cause any problem for $2 \rightarrow 2$ processes where the total cross section is obtained by integrating the matrix element with respect to only 
one variable, the scattering angle. When the final state contains more than 2-body, however, one needs to perform a multi-dimensional integration over phase space with, for example, 4 variables for $2 \rightarrow 3$ and 7 for $2 \rightarrow 4$ processes. If one relies on integration packages that are based on Monte-Carlo algorithms, one has to compute the loop matrix element many times, typically in the order of $50 \mathrm{~K}-1 \mathrm{M}$ times in total. To get the $\mathcal{O}(\alpha)$ corrections the contribution of the one-loop diagrams must be combined with the cross section for real photon emission. Usually the latter almost compensate the virtual corrections. Thus the number of points also should be properly chosen to achieve good accuracy. Hence, at least at present, it is not always realistic especially for complicated processes such as $2 \rightarrow 3$ and $2 \rightarrow 4$ processes to use the method based on the helicity amplitudes for the computation of the total cross section as this will require a lot of CPU time. The helicity amplitude approach is, however, helpful for comparing and checking, at several points in phase space, the squared matrix elements obtained the symbolic way, through REDUCE for example. Another limitation of the symbolic way, however, is that it cannot be applied to a process that has no tree-level contribution such as $\gamma \gamma \rightarrow \gamma \gamma$ and $\gamma \gamma \rightarrow \gamma Z$. For such cases one has to use the helicity amplitude approach.

\subsection{Regularisation scheme and the issue of $\gamma_{5}$}

An important step in the proof of renormalisability of the $\mathcal{S M}$ is that the symmetries that are present at tree-level are still preserved at the loop level. With a theory like the $\mathcal{S} \mathcal{M}$ that involves both vector and axial currents, it has been known that all gauge symmetries may not be preserved at the loop level due to the Adler-Bell-Jackiw anomaly [6]. In the case of the $\mathcal{S M}$, the charge assignments of all particles within a family is such that the anomaly is not present [77]. It is important to stress that the existence of a genuine anomaly has nothing to do with regularisation in the sense that there could be no regularisation that can make the anomaly disappear while maintaining all other quantum symmetries. Nonetheless an unfortunate choice of regularisation can induce an apparent anomaly that would violate some Ward identities that could stand in the way of a proper renormalisation programme. The use, even in QED, of a naive cut-off is such an example. These apparent anomalies can be removed by the introduction of extra counterterms usually not obtained through multiplicative renormalisation. This, of course, unduly complicates the renormalisation procedure and can make the implementation at the automation level more problematic. Pauli-Villars regularisation 78 that works so well in QED fails in the case of non-Abelian gauge theories because a mass term is introduced in a naive way. Dimensional Regularisation [38, 39] is a very powerful and extremely practical scheme for regularising divergences in non-Abelian theories ${ }^{6}$. In DR, the loop integrals as well as the Dirac and tensor algebra are calculated in a space-time with arbitrary $n$ dimensions. It is relatively easy to implement in an automatic code for one-loop calculations. The divergences are isolated as poles in $n-4$ through, for example, the variable $C_{U V}$ as is done in GRACE, with

$$
C_{U V}=\frac{1}{\varepsilon}-\gamma_{E}+\log 4 \pi, \quad n=4-2 \varepsilon .
$$

\footnotetext{
${ }^{6}$ For a step-by-step presentation of the method, see for example [79] or [80. The chiral anomaly is also nicely exposed in these textbooks.
} 
For the electroweak $\mathcal{S M}$, the problem with DR is the treatment of $\gamma_{5}$ which is a 4-dimensional object and hence can not be continued to an arbitrary $n$-dimension. With calculations involving $\gamma_{5}$ one therefore needs some additional scheme or prescription. In the original 't Hooft-Veltman prescription [38, 81], $\gamma_{5}$ anticommutes with the $\gamma$ matrices that carry one of the $n=4$ dimensions but commutes with the rest. This split is not satisfactory as it breaks Lorentz invariance in the full $n$-dimensional space and does not manifestly respect the conservation of the gauge current 82, 80. Many variations on the original scheme, that may be considered as not being fully mathematically consistent, have been applied in different calculations, see 83 for a review. For applications to the anomaly-free $\mathcal{S M}$, the most efficient and practical scheme, especially from the point of view of implementation in a computer code, is the so-called Naive Dimensional Regularisation whereby $\gamma_{5}$ is taken as fully anticommuting with all the $n$-dimensional $\gamma$ matrices. In GRACE-1oop this is what is implemented. Let us also point out that the cyclicity of the trace is not used. For loop calculations in supersymmetry DR is not quite appropriate since it breaks supersymmetry by splitting the number of fermionic and bosonic degrees of freedom. An alternative is Dimensional Reduction 84, 85]. A very nice review is given in [86, 87]. At the one-loop level an equivalent prescription is Constrained Differential Renormalisation [88, CDR. Reference [30, 31] shows how both DR and CDR can be implemented in the automatic code, FormCalc for the calculation of one-loop diagrams.

\subsection{Size of the generated programs}

One of the difficulties with running codes of one-loop processes produced by an automatic system has to do with the huge increase in the number of Feynman diagrams, as compared to tree-level processes, combined with the equally large size of the generated computer file for the matrix elements of each diagram. We could then easily end up with a total size of a program for a given process which is so huge that it is not always easy to compute the cross section in a realistic time scale. To give a feel for the size of such programs, we show in Table 1 examples for some processes that have been calculated so far with up to 6 -leg final states $(2 \rightarrow 4$ processes $)$. Here the total number of diagrams in a general non-linear gauge is shown. Once the gauge parameter check is successfully passed, for the cases of $2 \rightarrow 3$ and $2 \rightarrow 4$ processes the amplitudes are generated again in the linear gauge, which corresponds to a particular choice of the non-linear gauge as will be explained and by switching off the electron Yukawa coupling. This determines the set of amplitudes for the "production job" to be supplied to a phase space integrator. We note in passing that switching off the electron Yukawa coupling does not mean that all occurrences of the electron mass are set to zero. In particular the electron mass is kept in order to regularise collinear divergences, see section [8.3] or for the renormalisation of the fine structure constant in the charge counterterm, see Appendix [.

The size of the generated codes refers only to the amplitude and does not take into account common necessary libraries. In the case of 4 -fermion production, to save CPU time the masses of the light external fermions are neglected, as long as they appear in the numerator of the matrix element. At some points in phase space the full set and the production set are compared to confirm that the latter is correctly generated. The actual CPU time for $e^{+} e^{-} \rightarrow e^{+} e^{-} H$, for example, amounts to $150 \mathrm{~K}$ hours equivalent to a single IBM RS/6000(375MHz, Power 3) CPU in quadruple precision. 
Table 1: Size of the generated code for some cross sections measured by the number of lines. We also show the total number of tree-level and one-loop diagrams for each process in some general gauge. The numbers in parenthesis have the small electron Yukawa couplings switched off. "helicity" refers to the method of generating the helicity amplitude. REDUCE refers to the direct computation of the full matrix elements squared.

\begin{tabular}{|c|c|c|c|c|c|}
\hline \multirow{3}{*}{$2 \rightarrow n$-body } & process & \multicolumn{2}{|c|}{ number of graphs } & \multicolumn{2}{c|}{ program size } \\
\cline { 3 - 6 } & & tree & one-loop & $\begin{array}{c}\text { helicity } \\
\text { (lines) }\end{array}$ & $\begin{array}{c}\text { REDUCE } \\
\text { (lines) }\end{array}$ \\
\hline \multirow{2}{*}{$2 \rightarrow 2$} & $e^{+} e^{-} \rightarrow t \bar{t}$ & $4(2)$ & $150(54)$ & $48 \mathrm{~K}$ & $54 \mathrm{~K}$ \\
& $e^{+} e^{-} \rightarrow W^{+} W^{-}$ & $4(3)$ & $334(153)$ & $151 \mathrm{~K}$ & $282 \mathrm{~K}$ \\
& $W^{+} W^{-} \rightarrow W^{+} W^{-}$ & $7(7)$ & $925(921)$ & $432 \mathrm{~K}$ & $1.28 \mathrm{M}$ \\
\hline $2 \rightarrow 3$ & $e^{+} e^{-} \rightarrow \nu_{e} \bar{\nu}_{e} H[2]$ & $12(2)$ & $1350(249)$ & $92 \mathrm{~K}$ & $696 \mathrm{~K}$ \\
& $e^{+} e^{-} \rightarrow e^{+} e^{-} H[42]$ & $42(2)$ & $4470(510)$ & - & $154 \mathrm{~K}$ \\
& $e^{+} e^{-} \rightarrow t \bar{t} H[45]$ & $21(6)$ & $2327(771)$ & $581 \mathrm{~K}$ & $1.50 \mathrm{M}$ \\
& $e^{+} e^{-} \rightarrow Z H H[43]$ & $27(6)$ & $5417(1597)$ & - & $6.55 \mathrm{M}$ \\
& $e^{+} e^{-} \rightarrow \nu_{e} \bar{\nu}_{e} \gamma[\underline{[4}]$ & $10(5)$ & $1099(331)$ & $339 \mathrm{~K}$ & $1.06 \mathrm{M}$ \\
\hline $2 \rightarrow 4$ & $e^{+} e^{-} \rightarrow \nu_{e} \bar{\nu}_{e} H H$ & $81(12)$ & $19638(3416)$ & & $50.5 \mathrm{M}$ \\
& $e^{+} e^{-} \rightarrow \mu^{-} \bar{\nu}_{\mu} u \bar{d}[\underline{52}]$ & $44(10)$ & $6094(668)$ & $1.22 \mathrm{M}$ & $75.8 \mathrm{M}$ \\
\hline
\end{tabular}

\section{The Standard Model in a general non-linear gauge}

\subsection{The classical Lagrangian}

To help define our conventions and notations we first introduce the classical Lagrangian of the $\mathcal{S M}$ which is fully gauge invariant under $S U(2) \times U(1)$.

We denote the gauge fields of the theory of the $S U(2) \times U(1)$ group as $W_{\mu}^{a}(a=0,1,2,3)$. The weak isospin triplet refers to $1,2,3$ and the hypercharge singlet to the 0 component. The corresponding gauge couplings are $g^{a}$. The gauge invariant field strength $F_{\mu \nu}^{a}$ writes in a compact form as

$F_{\mu \nu}^{a}=\partial_{\mu} W_{\nu}^{a}-\partial_{\nu} W_{\mu}^{a}+g^{a} f^{a b c} W_{\mu}^{b} W_{\nu}^{c}, g^{a}=\left\{\begin{array}{ll}g & (a=1,2,3) \\ g^{\prime} & (a=0)\end{array}, f^{a b c}=\left\{\begin{array}{cc}\varepsilon^{a b c} & (a, b, c \neq 0) \\ 0 & \text { (otherwise) }\end{array}\right.\right.$.

This leads to the pure gauge contribution $\mathcal{L}_{G}$

$$
\mathcal{L}_{G}=-\frac{1}{4} F_{\mu \nu}^{a} F^{a \mu \nu} .
$$

The gauge interaction of the matter fields is completely specified by their isospin and hypercharge 
$(Y)$ quantum numbers, such that the electromagnetic charge is $Q=T^{3}+Y$, and the covariant derivative, $D_{\mu}$

$$
D_{\mu}=\partial_{\mu}-i g \sum_{a=1}^{3} W_{\mu}^{a} T^{a}-i g^{\prime} Y W_{\mu}^{0}
$$

with $T^{a}=\sigma^{a} / 2$, where $\sigma^{a}$ are the usual Pauli matrices. Left-handed fermions $L$ of each generation belong to a doublet while right-handed fermions $R$ are in a $S U(2)$ singlet. The fermionic gauge Lagrangian is just

$$
\mathcal{L}_{F}=i \sum \bar{L} \gamma^{\mu} D_{\mu} L+i \sum \bar{R} \gamma^{\mu} D_{\mu} R
$$

where the sum is assumed over all doublets and singlets of the three generations.

Mass terms for both the gauge bosons and fermions are generated in a gauge invariant way through the Higgs mechanism. To that effect one introduces a scalar doublet with hypercharge $Y=1 / 2$ that spontaneously breaks the symmetry of the vacuum through a non-zero vacuum expectation value $v$

$$
\phi=\frac{1}{\sqrt{2}}\left(\begin{array}{c}
i \chi_{1}+\chi_{2} \\
v+H-i \chi_{3}
\end{array}\right)=\left(\begin{array}{c}
i \chi^{+} \\
\left(v+H-i \chi_{3}\right) / \sqrt{2}
\end{array}\right), \quad\langle 0|\phi| 0\rangle=\left(\begin{array}{c}
0 \\
v / \sqrt{2}
\end{array}\right) .
$$

The scalar Lagrangian writes

$$
\mathcal{L}_{S}=\left(D_{\mu} \phi\right)^{\dagger}\left(D^{\mu} \phi\right)+\mathcal{L}_{\text {pot }}, \quad \mathcal{L}_{\text {pot }}=-V(\phi)=\mu^{2} \phi^{\dagger} \phi-\lambda\left(\phi^{\dagger} \phi\right)^{2} \equiv-\lambda\left(\phi^{\dagger} \phi-\frac{v^{2}}{2}\right)^{2}+\frac{\mu^{4}}{4 \lambda} .
$$

The Nambu-Goldstone bosons $\chi^{ \pm}, \chi_{3}$ in $\mathcal{L}_{S}$ get absorbed by the $Z$ and $W^{ \pm}$to give the latter masses $\left(M_{Z, W}\right)$, while the photon $A$ remains massless. The physical fields $W^{ \pm}, A, Z$ relate to the original $W$ quartet as

$$
\left\{\begin{array}{l}
A_{\mu}=\frac{g^{\prime} W_{\mu}^{3}+g W_{\mu}^{0}}{\sqrt{g^{2}+g^{\prime 2}}}=s_{W} W_{\mu}^{3}+c_{W} W_{\mu}^{0} \\
Z_{\mu}=\frac{g W_{\mu}^{3}-g^{\prime} W_{\mu}^{0}}{\sqrt{g^{2}+g^{\prime 2}}}=c_{W} W_{\mu}^{3}-s_{W} W_{\mu}^{0} \\
W_{\mu}^{ \pm}=\frac{W_{\mu}^{1} \mp i W_{\mu}^{2}}{\sqrt{2}}
\end{array}\right.
$$

with

$$
c_{W}=\frac{g}{\sqrt{g^{2}+g^{\prime 2}}}, \quad s_{W}=\frac{g^{\prime}}{\sqrt{g^{2}+g^{\prime 2}}}
$$


the electromagnetic coupling $e$

$$
e=\frac{g g^{\prime}}{\sqrt{g^{2}+g^{\prime 2}}}, \quad g=\frac{e}{s_{W}}, \quad g^{\prime}=\frac{e}{c_{W}},
$$

and the masses

$$
M_{W}=\frac{e v}{2 s_{W}}, \quad M_{Z}=\frac{e v}{2 s_{W} c_{W}} .
$$

$\mathcal{L}_{S}$ also defines the mass of the Higgs

$$
M_{H}^{2}=2 \mu^{2}
$$

$\mu, \lambda$ and $v$ are not all independent parameters. $v$ is defined to be the minimum of the scalar potential. This is equivalent to requiring no tadpole in $\mathcal{L}_{S}$. In other words we require the coefficient, $T$,

$$
T=v\left(\mu^{2}-\lambda v^{2}\right),
$$

of the term linear in $H$ in Eq. 5.6 to be zero, $T=0$. We will impose this requirement to all orders.

Fermion masses require the introduction of a corresponding Yukawa coupling, $f_{U}\left(f_{D}\right)$ for an up-type fermion $f$ (for a down-type fermion)

$$
\mathcal{L}_{M}=-\sum_{\text {up }} f_{U} \bar{L}_{U} \tilde{\phi} R_{U}-\sum_{\text {down }} f_{D} \bar{L}_{D} \phi R_{D}+(\text { h.c. }), \quad \tilde{\phi}=i \sigma^{2} \phi^{*}, m_{U, D}=\frac{f_{U, D} v}{\sqrt{2}} .
$$

Instead of the original set of independent parameters $\left\{g, g^{\prime}, \lambda, \mu^{2}, f_{U, D}\right\}$, it is much more advantageous to revert to an equivalent set of physical parameters that is directly related to physical observables, namely $\left\{e, M_{W}, M_{Z}, M_{H}, m_{U}, m_{D}\right\}$. In this respect note that the weak mixing angle is just a book-keeping quantity that will be defined, at all orders of perturbation, in terms of the masses of the vector bosons:

$$
c_{W}=\frac{M_{W}}{M_{Z}} .
$$

If one allows $v$ to be an independent parameter we will trade it for the tadpole, $T$, which we will add to the list of independent parameters that specify the theory.

\subsection{Quantisation: Gauge-fixing and Ghost Lagrangian}

As known because of the gauge freedom in the classical Lagrangian $\mathcal{L}_{C}, \mathcal{L}_{C}=\mathcal{L}_{G}+\mathcal{L}_{F}+\mathcal{L}_{S}+\mathcal{L}_{M}$, a Lorentz invariant quantisation requires a gauge fixing. We generalise the usual 't Hooft linear gauge condition to a more general non-linear gauge that involves five extra parameters [54, $\zeta=(\tilde{\alpha}, \tilde{\beta}, \tilde{\delta}, \tilde{\kappa}, \tilde{\epsilon})$.

$$
\mathcal{L}_{G F}=-\left.\frac{1}{\xi_{W}} \overbrace{\mid\left(\partial_{\mu}-i e \tilde{\alpha} A_{\mu}-i g c_{W} \tilde{\beta} Z_{\mu}\right) W^{\mu+}+\xi_{W}^{\prime} \frac{g}{2}\left(v+\tilde{\delta} H+i \tilde{\kappa} \chi_{3}\right) \chi^{+}}^{G^{+}}\right|^{2}
$$




$$
\begin{aligned}
& -\frac{1}{2 \xi_{Z}} \overbrace{\left(\partial . Z+\xi_{Z}^{\prime} \frac{g}{2 c_{W}}(v+\tilde{\varepsilon} H) \chi_{3}\right.}^{G^{Z}})^{2}-\frac{1}{2 \xi_{A}}(\overbrace{\partial . A}^{G_{A}})^{2} \\
\equiv & -\frac{1}{\xi_{W}} G^{+} G^{-}-\frac{1}{2 \xi_{Z}}\left(G^{Z}\right)^{2}-\frac{1}{2 \xi_{A}}\left(G^{A}\right)^{2}
\end{aligned}
$$

Note that it is not essential for the Feynman parameters $\xi_{W, Z}^{\prime}$ that appear within the functions $G^{ \pm, Z}$ to be equal to those that appear as factors of $G^{+} G^{-}\left(\xi_{W}\right)$ and $G_{Z}^{2}\left(\xi_{Z}\right)$. However in this case $\mathcal{L}_{G F}$ does not cancel, at tree-level, the mixing terms $\chi-W, \chi_{3}-Z$, see for instance 80, 89. To avoid this unnecessary complications we will stick to $\xi_{W, Z}^{\prime}=\xi_{W, Z}$.

To construct the ghost Lagrangian $\mathcal{L}_{G h}$, we will require that the full effective Lagrangian, or rather the full action, be invariant under the BRST transformation (the measure being invariant). The required set of transformations needed to construct the ghost Lagrangian together with the definition of the ghost fields can be found in Appendix A. This is a much more appropriate procedure than the usual Fadeev-Popov approach especially when dealing with the quantum symmetries of the generalised non-linear gauges we are studying. This implies that the full quantum Lagrangian

$$
\mathcal{L}_{Q}=\mathcal{L}_{C}+\mathcal{L}_{G F}+\mathcal{L}_{G h},
$$

be such that $\delta_{\mathrm{BRS}} \mathcal{L}_{Q}=0$ and therefore $\delta_{\mathrm{BRS}} \mathcal{L}_{G F}=-\delta_{\mathrm{BRS}} \mathcal{L}_{G h}$.

Moreover we appeal to the auxiliary $B$-field formulation of the gauge-fixing Lagrangian $\mathcal{L}_{G F}$. We will see later that this formulation is also very useful to extract some Ward-Takahashi identities. Within this approach

$$
\mathcal{L}_{G F}=\xi_{W} B^{+} B^{-}+\frac{\xi_{Z}}{2}\left|B^{Z}\right|^{2}+\frac{\xi_{A}}{2}\left|B^{A}\right|^{2}+B^{-} G^{+}+B^{+} G^{-}+B^{Z} G^{Z}+B^{A} G^{A} .
$$

From the equations of motion for the $B$-fields one recovers the usual $\mathcal{L}_{G F}$ together with the condition $B^{i}=-\frac{G^{i}}{\xi_{i}}\left(\xi=\left\{\xi_{W}, \xi_{Z}, \xi_{A}\right\}\right)$.

Defining the anti-ghost, $\bar{c}^{i}$, from the gauge fixing functions, we write

$$
\delta_{\mathrm{BRS}} \bar{c}^{i}=B^{i} .
$$

Then by identification

$$
\begin{aligned}
\mathcal{L}_{G h} & =-\left(\bar{c}^{+} \delta_{\mathrm{BRS}} G^{+}+\bar{c}^{-} \delta_{\mathrm{BRS}} G^{-}+\bar{c}^{Z} \delta_{\mathrm{BRS}} G^{Z}+\bar{c}^{A} \delta_{\mathrm{BRS}} G^{A}\right)+\delta_{\mathrm{BRS}} \tilde{\mathcal{L}}_{G h} \\
& \equiv \mathcal{L}_{F P}+\delta_{\mathrm{BRS}} \tilde{\mathcal{L}}_{G h} .
\end{aligned}
$$

That is, one recovers the Fadeev-Popov prescription, $\mathcal{L}_{F P}$, but only up to an overall function, $\delta_{\mathrm{BRS}} \tilde{\mathcal{L}}_{G h}$, which is BRST invariant. The complete Feynman rules we list assume $\tilde{\mathcal{L}}_{G h}=0$ which is sufficient for one-loop calculations. For higher orders a counterterm not of the Fadeev-Popov type, but which is BRST invariant on its own, may be required to renormalise a quartic ghost vertex, in this case one can take $\tilde{\mathcal{L}}_{G h}=\lambda \epsilon_{i j k} \delta_{\mathrm{BRS}}\left(\bar{c}^{i} \bar{c}^{j} c^{k}\right)$. The full set of Feynman rules derived 
from $\mathcal{L}_{Q}$ is relegated to Appendix $\mathbb{B}$ These Feynman rules are derived with an arbitrary set $\zeta=(\tilde{\alpha}, \tilde{\beta}, \tilde{\delta}, \tilde{\kappa}, \tilde{\epsilon}), \xi_{i}=\left(\xi_{W}, \xi_{Z}, \xi_{A}\right)$ although for one-loop applications and for all our tests we stick with the 't Hooft-Feynman gauge $\xi_{W}=\xi_{Z}=\xi_{A}=1$ where the gauge boson propagators take a very simple form. Only their "transverse" part $g_{\mu \nu}$ contributes, see Eq. 1.1. This also greatly simplifies the calculations not only because the expressions get more compact but also because of the fact that the longitudinal parts introduce a high degree of (superficial) ultraviolet divergences. Although these greatly simplify when adding, all diagrams the cancellations are very subtle and may be not efficiently handled when implemented numerically. In practical calculations one can also tune $\tilde{\alpha}, \tilde{\beta}, .$. so that one reduces the number of diagrams and simplify some of the vertices. For instance for photonic vertices $\tilde{\alpha}=1$ is to be preferred since there is no $W^{ \pm} \chi^{\mp} A$ vertex and also because the $W W \gamma$ simplifies considerably. One can also choose $\tilde{\beta}$ so that $W^{ \pm} \chi^{\mp} Z$ vanishes.

\section{Renormalisation and counterterms}

\subsection{Renormalisation constants}

The renormalisation procedure follows very closely the on-shell renormalisation scheme, carried in 90] in the case of the usual linear gauge. The set of physical input parameters includes all the masses of the model together with the value of the electromagnetic coupling as defined in the Thomson limit. As explained above we also add the tadpole, $T$, to this list. Renormalisation of these parameters would then lead to finite S-matrix elements. For the mass eigenstates and thus a proper identification of the physical particles that appear as external legs in our processes, field renormalisation is needed. S-matrix elements obtained from these rescaled Green's functions will lead to external legs with unit residue. Therefore one also needs wave function renormalisation of the fields. In the linear gauge with all $\xi=1$ this also renders Green's functions finite. Especially for the unphysical sector of the theory, the precise choice of the fields redefinition is not essential if one is only interested in S-matrix elements of physical processes. We will therefore concentrate essentially on the renormalisation of the physical parameters and physical fields, although we also introduce field renormalisation for the Goldstone bosons.

All fields and parameters introduced so far in section 3 are considered as bare parameters with the exception of the gauge fixing Lagrangian which we choose to write in terms of renormalised fields. Care should then be exercised when we split the tree-level contributions and the counterterms. In Appendix $\mathrm{E}$ we also present the alternative approach where the gauge-fixing term is also written in terms of bare parameters. Differences between the two approaches, of course, only affect the unphysical scalar sector. In Appendix D we derive some useful Ward identities that constrain the two-point functions in this sector.

For the renormalised quantity $X$, the corresponding bare value will be defined by an underlined - symbol, $\underline{X}$, and its counterterm by $\delta X$.

For the physical parameters, and the tadpole, we define

$$
\underline{M}_{W}^{2}=M_{W}^{2}+\delta M_{W}^{2}
$$




$$
\begin{aligned}
\underline{M}_{Z}^{2} & =M_{Z}^{2}+\delta M_{Z}^{2}, \\
\underline{m}_{f} & =m_{f}+\delta m_{f}, \\
\underline{M}_{H}^{2} & =M_{H}^{2}+\delta M_{H}^{2}, \\
\underline{e} & =Y e=(1+\delta Y) e, \\
\underline{T} & =T+\delta T .
\end{aligned}
$$

We now turn to the fields and the wave function renormalisation constants.

1. Gauge fields

$$
\begin{aligned}
& \underline{W}_{\mu}^{ \pm}=\sqrt{Z}_{W} W_{\mu}^{ \pm}, \sqrt{Z}_{W}=1+\delta Z_{W}^{1 / 2}, \\
& \left(\begin{array}{c}
\underline{Z}_{\mu} \\
\underline{A}_{\mu}
\end{array}\right)=\left(\begin{array}{cc}
\sqrt{Z}_{Z Z} & \sqrt{Z}_{Z A} \\
\sqrt{Z}_{A Z} & \sqrt{Z}_{A A}
\end{array}\right)\left(\begin{array}{c}
Z_{\mu} \\
A_{\mu}
\end{array}\right), \\
& \sqrt{Z}_{A A, Z Z}=1+\delta Z_{A A, Z Z}^{1 / 2}, \quad \sqrt{Z}_{A Z, Z A}=\delta Z_{A Z, Z A}^{1 / 2} .
\end{aligned}
$$

\section{Fermions}

For simplicity we will assume no fermion mixing and therefore no $\mathcal{C P}$ violation. In this case the wave functions renormalisation constants $\delta Z_{f L, f R}^{1 / 2}$ can be taken real as we will see later.

$$
\stackrel{(-)}{f}_{L, R}=\sqrt{Z}_{f_{L, R}} \stackrel{(-)}{f}_{L, R}, \quad \sqrt{Z}_{f_{L, R}}=1+\delta Z_{f_{L, R}}^{1 / 2} .
$$

3. Scalars

$$
\underline{S}=\sqrt{Z}_{S} S, \sqrt{Z}_{S}=1+\delta Z_{S}^{1 / 2}, S=H, \chi^{ \pm}, \chi_{3} .
$$

Because we are only presenting an application to processes at one-loop, there is no need to be specific about the renormalisation of the ghost sector. This is sketched in Appendix C. Suffice to say that to generate the ghost Lagrangian including counterterms one needs to re-express $\mathcal{L}_{G F}$ in terms of bare fields to first generate, through BRST transformations, $\mathcal{L}_{G h}$ with bare fields. This is because $\mathcal{L}_{G F}$ is written in terms of renormalised fields and as such does not induce any counterterm. However the BRST transformations are defined for bare fields.

The generated counterterm library for all 3 and 4-point vertices is listed in Appendix [F The counterterms are fixed through renormalisation conditions that are set, with the exception of the $e^{+} e^{-} A$ vertex $^{7}$, from the two-point functions to which we now turn.

\subsection{Two-point functions at one-loop including counterterms}

We work in the on-shell scheme closely following [90] for the determination of the renormalisation constants. The renormalisation conditions on the parameters are essentially derived by properly defining the masses of all the physical particles $A, Z, W^{ \pm}, f$ and the electromagnetic constant.

\footnotetext{
${ }^{7}$ In fact, as we will see, due to a Ward identity, the counterterm for the charge can be expressed in terms of two-point functions also, see Eq. 6.23
} 
They are all set from the propagator and the $e^{+} e^{-} A$ vertex. Let us first turn to the propagators of the fields of the theory.

The counterterm contribution will be denoted by a caret while the full contribution (counterterm and one-loop diagrams contribution) is denoted by a tilde, so that for the vector boson we may write

$$
\tilde{\Pi}=\Pi+\hat{\Pi} .
$$

Moreover it is necessary to decompose these contributions according to their Lorentz structure. For our purpose we will only consider the case of no mixing (and hence no $\mathcal{C P}$ violation) in the fermionic sector. The decomposition of two-point functions is as follows.

\begin{tabular}{ll}
\hline type & formula \\
\hline vector-vector & $\Pi_{\mu \nu}\left(q^{2}\right)=\left(g_{\mu \nu}-\frac{q_{\mu} q_{\nu}}{q^{2}}\right) \Pi_{T}\left(q^{2}\right)+\frac{q_{\mu} q_{\nu}}{q^{2}} \Pi_{L}\left(q^{2}\right)$ \\
\hline scalar-scalar & $\Pi\left(q^{2}\right)$ \\
\hline vector-scalar & $i q_{\mu} \Pi\left(q^{2}\right)(q$ is the momentum of the incoming scalar $)$ \\
\hline fermion-fermion & $\Sigma\left(q^{2}\right)=K_{1} I+K_{5} \gamma_{5}+K_{\gamma} \not+K_{5 \gamma} \phi \gamma_{5}$ \\
\hline
\end{tabular}

Complete one-loop results for all two-point functions in the generalised non-linear gauge are collected in Appendix The contribution of the counterterms to the two-point functions writes

1. Vector-Vector

\begin{tabular}{ll}
\hline$W W$ & $\hat{\Pi}_{T}^{W}=\delta M_{W}^{2}+2\left(M_{W}^{2}-q^{2}\right) \delta Z_{W}^{1 / 2}$ \\
& $\hat{\Pi}_{L}^{W}=\delta M_{W}^{2}+2 M_{W}^{2} \delta Z_{W}^{1 / 2}$ \\
\hline$Z Z$ & $\hat{\Pi}_{T}^{Z Z}=\delta M_{Z}^{2}+2\left(M_{Z}^{2}-q^{2}\right) \delta Z_{Z Z}^{1 / 2}$ \\
& $\hat{\Pi}_{L}^{Z Z}=\delta M_{Z}^{2}+2 M_{Z}^{2} \delta Z_{Z Z}^{1 / 2}$ \\
\hline$Z A$ & $\hat{\Pi}_{T}^{Z A}=\left(M_{Z}^{2}-q^{2}\right) \delta Z_{Z A}^{1 / 2}-q^{2} \delta Z_{A Z}^{1 / 2}$ \\
& $\hat{\Pi}_{L}^{Z A}=M_{Z}^{2} \delta Z_{Z A}^{1 / 2}$ \\
\hline$A A$ & $\hat{\Pi}_{T}^{A A}=-2 q^{2} \delta Z_{A A}^{1 / 2}$ \\
& $\hat{\Pi}_{L}^{A A}=0$ \\
\hline
\end{tabular}

2. Scalar-Scalar

\begin{tabular}{ll}
\hline$H H$ & $\hat{\Pi}^{H}=2\left(q^{2}-M_{H}^{2}\right) \delta Z_{H}^{1 / 2}-\delta M_{H}^{2}+\frac{3 \delta T}{v}$ \\
\hline$\chi_{3} \chi_{3}$ & $\hat{\Pi}^{\chi_{3}}=2 q^{2} \delta Z_{\chi_{3}}^{1 / 2}+\frac{\delta T}{v}$ \\
\hline$\chi \chi$ & $\hat{\Pi}^{\chi}=2 q^{2} \delta Z_{\chi}^{1 / 2}+\frac{\delta T}{v}$ \\
\hline
\end{tabular}


3. Vector-Scalar

$$
\begin{array}{ll}
\hline W \chi & \hat{\Pi}^{W \chi}=M_{W}\left(\delta M_{W} / M_{W}+\delta Z_{W}^{1 / 2}+\delta Z_{\chi}^{1 / 2}\right) \\
\hline Z \chi_{3} & \hat{\Pi}^{Z \chi_{3}}=M_{Z}\left(\delta M_{Z} / M_{Z}+\delta Z_{Z Z}^{1 / 2}+\delta Z_{\chi_{3}}^{1 / 2}\right) \\
\hline A \chi_{3} & \hat{\Pi}^{A \chi_{3}}=M_{Z} \delta Z_{Z A}^{1 / 2} \\
\hline
\end{array}
$$

4. Fermion-Fermion

At one-loop, this sector is unaffected by the parameters of the non-linear gauge and thus all functions are as in the usual linear gauge case. As mentioned earlier, all wave functions constants are real since we do not consider mixing in the fermionic sector.

$$
\begin{aligned}
\hat{K}_{1} & =-m_{f}\left(\delta Z_{f L}^{1 / 2}+\delta Z_{f R}^{1 / 2}\right)-\delta m_{f}, \\
\hat{K}_{5} & =0 \\
\hat{K}_{\gamma} & =\left(\delta Z_{f L}^{1 / 2}+\delta Z_{f R}^{1 / 2}\right) \\
\hat{K}_{5 \gamma} & ==-\left(\delta Z_{f L}^{1 / 2}-\delta Z_{f R}^{1 / 2}\right) .
\end{aligned}
$$

\subsection{Renormalisation Conditions}

Leaving aside the renormalisation of the electromagnetic charge, these two-point functions give all other renormalisation constants. Before deriving these let us first turn to the tadpole.

1. Tadpole

The counterterm for the tadpole contribution, $\delta T$, is defined such that the tadpole loop contribution $T^{\text {loop }}$ and the counterterm $\delta T$ combine such that the tadpole $\tilde{T}=T^{\text {loop }}+$ $\delta T=0$. Then

$$
\delta T=-T^{l o o p} .
$$

The tadpole contribution in the electroweak $\mathcal{S M}$ is sometimes necessary. An example is the loop two-point functions of the massive vector bosons and the Higgs in order to check the BRST 65, 66] or the Slavnov-Taylor 91 identities, see for example Appendix D.

2. Charged vector

The conditions specify that the pole-position of the propagator is $M_{W}^{2}$, and that the residue of the propagator at the pole is 1 .

$$
\Re e \tilde{\Pi}_{T}^{W}\left(M_{W}^{2}\right)=0,\left.\quad \frac{d}{d q^{2}} \Re e \tilde{\Pi}_{T}^{W}\left(q^{2}\right)\right|_{q^{2}=M_{W}^{2}}=0 .
$$

This gives the following relations.

$$
\delta M_{W}^{2}=-\Re e \Pi_{T}^{W}\left(M_{W}^{2}\right), \quad \delta Z_{W}^{1 / 2}=\left.\frac{1}{2} \frac{d}{d q^{2}} \Re e \Pi_{T}^{W}\left(q^{2}\right)\right|_{q^{2}=M_{W}^{2}} .
$$




\section{Neutral vector}

The conditions to be imposed on the photon-photon and $Z-Z$ self-energies are the same as for the $W-W$ transition. In addition we require that there should be no mixing between $Z$ and the photon at the poles $q^{2}=0, M_{Z}^{2}$.

$$
\begin{gathered}
\Re e \tilde{\Pi}_{T}^{Z Z}\left(M_{Z}^{2}\right)=0,\left.\quad \frac{d}{d q^{2}} \Re e \tilde{\Pi}_{T}^{Z Z}\left(q^{2}\right)\right|_{q^{2}=M_{W}^{2}}=0, \\
\tilde{\Pi}_{T}^{A A}(0)=0,\left.\quad \frac{d}{d q^{2}} \tilde{\Pi}_{T}^{A A}\left(q^{2}\right)\right|_{q^{2}=0}=0 \\
\tilde{\Pi}_{T}^{Z A}(0)=0, \quad \Re e \tilde{\Pi}_{T}^{Z A}\left(M_{Z}^{2}\right)=0 .
\end{gathered}
$$

Among these 6 conditions, $\tilde{\Pi}_{T}^{A A}(0)=0$ produces nothing, except that it ensures that the loop calculation does indeed give $\Pi_{T}^{A A}(0)=0$. One then derives,

$$
\begin{gathered}
\delta M_{Z}^{2}=-\Re e \Pi_{T}^{Z Z}\left(M_{Z}^{2}\right), \quad \delta Z_{Z Z}^{1 / 2}=\left.\frac{1}{2} \Re e \frac{d}{d q^{2}} \Pi_{T}^{Z Z}\left(q^{2}\right)\right|_{q^{2}=M_{Z}^{2}}, \\
\delta Z_{A A}^{1 / 2}=\frac{1}{2} \frac{d}{d q^{2}} \Pi_{T}^{A A}(0) \\
\delta Z_{Z A}^{1 / 2}=-\Pi_{T}^{Z A}(0) / M_{Z}^{2}, \quad \delta Z_{A Z}^{1 / 2}=\Re e \Pi_{T}^{Z A}\left(M_{Z}^{2}\right) / M_{Z}^{2}
\end{gathered}
$$

4. Higgs

The conditions specify that the pole-position of the propagator is $M_{H}^{2}$, and that the residue of the propagator at the pole is 1 ,

$$
\Re e \tilde{\Pi}^{H}\left(M_{H}^{2}\right)=0,\left.\quad \frac{d}{d q^{2}} \Re e \tilde{\Pi}^{H}\left(q^{2}\right)\right|_{q^{2}=M_{H}^{2}}=0 .
$$

This gives the following relations.

$$
\delta M_{H}^{2}=\Re e \Pi^{H}\left(M_{H}^{2}\right)+\frac{3 \delta T}{v}, \quad \delta Z_{H}^{1 / 2}=-\left.\frac{1}{2} \frac{d}{d q^{2}} \Re e \Pi^{H}\left(q^{2}\right)\right|_{q^{2}=M_{H}^{2}} .
$$

\section{Fermion}

The conditions for pole-positions and residues are the same as for the other physical particles. Also the vanishing of $\gamma_{5}$ and $\gamma^{\mu} \gamma_{5}$ terms at the pole is required. These conditions read

$$
\begin{gathered}
m_{f} \Re e \tilde{K}_{\gamma}\left(m_{f}^{2}\right)+\Re e \tilde{K}_{1}\left(m_{f}^{2}\right)=0,\left.\quad \frac{d}{d \phi} \Re e\left(d \tilde{K}_{\gamma}\left(q^{2}\right)+\tilde{K}_{1}\left(q^{2}\right)\right)\right|_{q=m_{f}}=0, \\
\Re e \tilde{K}_{5}\left(m_{f}^{2}\right)=0, \quad \Re e \tilde{K}_{5 \gamma}\left(m_{f}^{2}\right)=0 .
\end{gathered}
$$


$\mathcal{C P}$ invariance leads to $K_{5}=0$. In this case, one can take both $\delta Z_{f L}^{1 / 2}$ and $\delta Z_{f R}^{1 / 2}$ to be real using the invariance under a phase rotation. We obtain the following relations.

$$
\begin{aligned}
& \delta m_{f}=\Re e\left(m_{f} K_{\gamma}\left(m_{f}^{2}\right)+K_{1}\left(m_{f}^{2}\right)\right), \\
& \delta Z_{f L}^{1 / 2}=\frac{1}{2} \Re e\left(K_{5 \gamma}\left(m_{f}^{2}\right)-K_{\gamma}\left(m_{f}^{2}\right)\right)-\left.m_{f} \frac{d}{d q^{2}}\left(m_{f} \Re e K_{\gamma}\left(q^{2}\right)+\Re e K_{1}\left(q^{2}\right)\right)\right|_{q^{2}=m_{f}^{2}}, \\
& \delta Z_{f R}^{1 / 2}=-\frac{1}{2} \Re e\left(K_{5 \gamma}\left(m_{f}^{2}\right)+K_{\gamma}\left(m_{f}^{2}\right)\right)-\left.m_{f} \frac{d}{d q^{2}}\left(m_{f} \Re e K_{\gamma}\left(q^{2}\right)+\Re e K_{1}\left(q^{2}\right)\right)\right|_{q^{2}=m_{f}^{2}} .
\end{aligned}
$$

6. Charge

While there are many vertices in the theory, if the charge $e$ is properly renormalised, we do not need any further renormalisation conditions. The condition can be imposed on any vertex. The most natural one is to fix the $e^{+} e^{-} A$ vertex as is usually done in QED by relating it to the Thomson limit. The condition requests that the coupling is $-e$ when $q$, the momentum of the photon, is 0 , while the $e^{ \pm}$with momentum $p_{ \pm}$are one shell,

$$
\left.\left(e^{+} e^{-} A \text { one loop term }+e^{+} e^{-} A \text { counter term }\right)\right|_{q=0, p_{ \pm}^{2}=m_{e}^{2}}=0 .
$$

The counterterm is defined in Appendix [F] From this, we obtain $\delta Y$. In fact we will see that due to a Ward identity, see for example [92], $\delta Y$ writes as a combination of $\delta Z_{A A}^{1 / 2}$ and $\delta Z_{Z A}^{1 / 2}$ which is valid in all gauges.

7. The unphysical sector

Because we are interested in applications to physical processes, the renormalisation of this sector is not adamant. Nonetheless one may choose to work, as far as possible, with finite Green's functions involving the Goldstones and the longitudinal modes of the vector bosons. With a linear gauge-fixing condition in the 't Hooft-Feynman gauge, and in the approach we are taking where the gauge-fixing Lagrangian is written in terms of renormalised fields from the outset, all divergences in this sector are taken care of by properly choosing $Z_{\chi_{3}, \chi^{ \pm}}$. Therefore, following [90], we define the wave-function renormalisation for $\chi=\chi_{3}, \chi^{ \pm}$,

$$
\delta Z_{\chi}^{1 / 2}=-\left.\frac{1}{2} \frac{d}{d q^{2}}\left(\Pi^{\chi}\left(q^{2}\right)\right)\right|_{C_{U V}-\text { part }}
$$

where $\left.\Pi^{\chi}\left(q^{2}\right)\right|_{C_{U V}-\text { part }}$ is the divergent part of the Goldstone two-point functions. We extend the same definition in the case of the non-linear gauge, see for example Eq. E.10. In our approach, where the gauge fixing term is expressed in terms of renormalised quantities from the onset, this is not sufficient to make all the unphysical scalar two-point functions and mixing finite in the non-linear gauge. In fact in our approach and with the nonlinear gauge, even $\tilde{\Pi}_{L}^{W^{ \pm}}$, which does not involve $\delta Z_{\chi^{ \pm}}^{1 / 2}$, is not finite. However as shown in Appendix D there is a strong constraint on the two-point functions of the unphysical scalars. For our purpose of using these kinds of gauge fixing to check the gauge-parameter 
independence of the results, this also is a non trivial test on the finiteness and gauge independence of the results. In Appendix $\mathbb{E}$ we show explicitly how one may choose to have finite two-point functions in the Goldstone sector at the expense of renormalising the gauge parameters. This method could be followed but it introduces a few extra renormalisation constants, which may slow the code for the cross section evaluation. In any case, although we can calculate the cross sections in any gauge, the gauge-parameter independence check is systematically applied on some random points in phase space. When this is passed we generally calculate the cross section in the linear gauge, with all 't HooftFeynman parameters being equal to one. In this case, linear gauge condition with $\xi=1$, both approaches are equivalent.

\subsection{Some remarks on the explicit form of the renormalisation constants}

The renormalisation procedure outlined above together with the exact and complete computation of all two-point functions permits to derive in a straightforward manner the explicit expressions for all parameters and wave function renormalisation constants. The complete expressions for all two-point functions are defined in Appendix [H] From the conditions imposed in section 6.3 one immediately extracts all the necessary counterterms. Since the general expressions for these are lengthy and can be read off from Appendix $\mathbf{H}$ we do not list all of them here, but just comment on some important general features.

\subsubsection{Mass shifts and charge renormalisation}

We first verify that all counterterms to the input parameter of the physical particles, namely the masses of all particles, $\delta M_{W, Z, H, f}$, are gauge-parameter independent. This also applies to the tadpole counterterm. This constitutes a strong check on our results.

The same holds for the charge renormalisation constant, $\delta Y$. Although this may be derived from the knowledge of $\delta Z_{A A}^{1 / 2}$ and $\delta Z_{Z A}^{1 / 2}$ through a Ward identity, it is easy to compute it directly. This is done explicitly in Appendix [1] We find the gauge-parameter independent result

$$
\delta Y=-\delta Z_{A A}^{1 / 2}+\frac{s_{W}}{c_{W}} \delta Z_{Z A}^{1 / 2}
$$

While both $\delta Z_{A A}^{1 / 2}$ and $\delta Z_{Z A}^{1 / 2}$ are gauge-parameter dependent, see Eqs. 6.256.26 below, the above combination is universal.

$$
\delta Y=\frac{\alpha}{4 \pi}\left\{-\frac{7}{2}\left(C_{U V}-\log M_{W}^{2}\right)-\frac{1}{3}+\frac{2}{3} \sum_{f} Q_{f}^{2}\left(C_{U V}-\log m_{f}^{2}\right)\right\} .
$$

$C_{U V}$ is defined in Eq. 4.9.

\subsubsection{Wave function renormalisation constants}

Since $\delta Z_{A A}^{1 / 2}$ and $\delta Z_{Z A}^{1 / 2}$ are crucial for charge renormalisation and since their expressions are rather simple we give them explicitly. 
We have

$$
\begin{aligned}
\delta Z_{A A}^{1 / 2} & =\frac{\alpha}{4 \pi}\left[\left(\frac{3}{2}+2 \tilde{\alpha}\right)\left(C_{U V}-\log M_{W}^{2}\right)+\frac{1}{3}-\frac{2}{3} \sum_{f} Q_{f}^{2}\left(C_{U V}-\log m_{f}^{2}\right)\right] \\
& \equiv \frac{\alpha}{4 \pi}\left[\left(-2(1-\tilde{\alpha})+\frac{7}{2}\right)\left(C_{U V}-\log M_{W}^{2}\right)+\frac{1}{3}-\frac{2}{3} \sum_{f} Q_{f}^{2}\left(C_{U V}-\log m_{f}^{2}\right)\right]
\end{aligned}
$$

where a summation on all fermions of charge $Q_{f}$ is performed.

$$
\delta Z_{Z A}^{1 / 2}=-\frac{\alpha}{2 \pi} \frac{c_{W}}{s_{W}}(1-\tilde{\alpha})\left(C_{U V}-\log M_{W}^{2}\right) .
$$

This shows that although both $\delta Z_{A A}^{1 / 2}$ and $\delta Z_{Z A}^{1 / 2}$ are gauge-parameter dependent, the combination that appears in the charge renormalisation is not. Moreover, observe that the choice $\tilde{\alpha}=1$ gives a vanishing $Z$ - $A$ transition at one-loop. This is due to the residual $U(1)$ gauge symmetry which remains after gauge fixing the charged sector, with this particular choice of the gauge parameter.

The remaining wave functions constants are not very illuminating and involve lengthy expressions that we can extract from Appendix [H] Here we just list the gauge-parameter dependence of those of the physical particles which can be expressed in a rather compact form as

$$
\begin{aligned}
\delta Z_{A Z}^{1 / 2} & =\widetilde{Z_{A Z}^{1 / 2}}+\frac{\alpha}{2 \pi} \frac{c_{W}}{s_{W}} \tilde{\beta}\left(C_{U V}-\Re e F_{0}(W, W, Z)\right), \\
\delta Z_{Z Z}^{1 / 2} & =\widetilde{\delta Z_{Z Z}^{1 / 2}}+\frac{\alpha}{2 \pi} \frac{c_{W}^{2}}{s_{W}^{2}} \tilde{\beta}\left(C_{U V}-\Re e F_{0}(W, W, Z)\right), \\
\delta Z_{W}^{1 / 2} & =\widetilde{\delta Z_{W}^{1 / 2}}-\frac{\alpha}{4 \pi} \frac{1}{s_{W}^{2}}\left\{s_{W}^{2} \tilde{\alpha}\left(C_{U V}-\Re e F_{0}(A, W, W)\right)+c_{W}^{2} \tilde{\beta}\left(C_{U V}-\Re e F_{0}(Z, W, W)\right)\right\}, \\
\delta Z_{H}^{1 / 2} & =\widetilde{\widetilde{Z_{H}^{1 / 2}}}+\frac{\alpha}{8 \pi} \frac{1}{s_{W}^{2}}\left\{\tilde{\delta}\left(C_{U V}-\Re e F_{0}(W, W, H)\right)+\frac{\tilde{\epsilon}}{2 c_{W}^{2}}\left(C_{U V}-\Re e F_{0}(Z, Z, H)\right)\right\} .
\end{aligned}
$$

where the quantities with $\sim$ correspond to the linear gauge result with all Feynman parameters set to 1. The function $F_{0}$ is defined in Appendix G As known 93, 94, 95] the requirement of having the residues of the renormalised propagators of all physical particles to be unity leads to a (very sharp) threshold singularity in the wave function of the Higgs at the thresholds corresponding to $M_{H}=2 M_{W}, 2 M_{Z}$. This singularity is all contained in the explicit derivative term in $\delta Z_{H}^{1 / 2}$ and is therefore gauge-parameter independent. Solutions to smooth this behaviour [96], like the inclusion of the finite width of the $W$ and $Z$, do exist but we have not implemented them yet in the present version of GRACE. Therefore when scanning over $M_{H}$ it is sufficient to avoid these regions within $1 \mathrm{GeV}$ around the thresholds. 


\subsection{Issues of renormalisation for unstable particles}

\subsubsection{Wave function renormalisation for unstable particles: absorptive part and gauge dependence}

As can be explicitly seen in the previous paragraph wave function renormalisation constants, contrary to the counterterms for the physical parameters such as the masses and couplings, are gauge-parameter dependent. This is a reflection that fields are not physical observables. In fact, since at the end we are only interested in S-matrix observables, we could have defined an approach where we could have done without the introduction of wave function renormalisation but at the expense of not having finite Green's functions ${ }^{8}$. We discussed this aspect when we defined the wave function renormalisation of the unphysical Goldstone particles in section [6.3 and we argued that the wave function renormalisation of the Goldstones, which only appear as internal particles, cancels out exactly. The argument applies to all particles that only appear as internal particles. Indeed, the wave function renormalisation constant would appear both in the correction to the propagator and to the two vertices to which this propagator attaches. It is easy to see that the effect from the propagator cancels that from the vertices. The wave function renormalisation therefore only applies to the external particles. However there is a problem when one is dealing with the wave function renormalisation of unstable particles. One aspect of this problem was just pointed out in the previous paragraph, section 6.4 related to the singularity brought about by the wave function renormalisation of the Higgs near the threshold for $W W$ and $Z Z$ production. The other problem has to do with the fact that the wave function renormalisation has been defined to be real so that the Lagrangian be Hermitian. But obviously one is, for unstable particles, applying Hermitian quantum field theory to non-Hermitian problems 97 .

In the standard on-shell approach, because of wave function renormalisation, loop and counterterm insertions on external legs are simply and conveniently not taken into account since they are thought to cancel each other. The fact is, if one insists on only using the real part of the wave function renormalisation then one does not completely cancel the self-energy and counterterm insertions on the external legs of an unstable particle. There remains in particular a contribution from the absorptive part of the self-energy. These absorptive parts occur for unstable massive particles when thresholds are crossed, they could also correspond to unphysical thresholds that occur in gauges where the gauge parameter is $\xi \neq 1$.

Let us show an example where a gauge dependent threshold appears explicitly even for physical thresholds in the non-linear gauge. We take again a Higgs heavy enough to decay into a pair of $W$ and consider the one-loop amplitude. In particular we concentrate here only on the eventual $\tilde{\delta}$ dependence. This is contained in the two selected graphs in Fig. 12 consisting of the fish-type one-loop diagram and the $H W W$ counterterm.

The fish-type diagram develops a $\tilde{\delta}$ dependent absorptive part which, at the amplitude level, does not cancel against the counterterm contribution if one insists on real wave function renormalisation for the Higgs as given by Eq. 6.27. The absorptive $\tilde{\delta}$ dependent contribution from the fish would cancel on the other hand if $\delta Z_{H}$ is defined to contain both the real and

\footnotetext{
${ }^{8}$ In fact, as stated in section 6.3 not all two-point functions and mixing involving the unphysical scalars are finite in the general non-linear gauge in our approach starting with a renormalised gauge-fixing term.
} 
Figure 12: Selected $\tilde{\delta}$ dependent diagrams for $\chi^{ \pm}$loop(left) and the vertex counterterm(right).

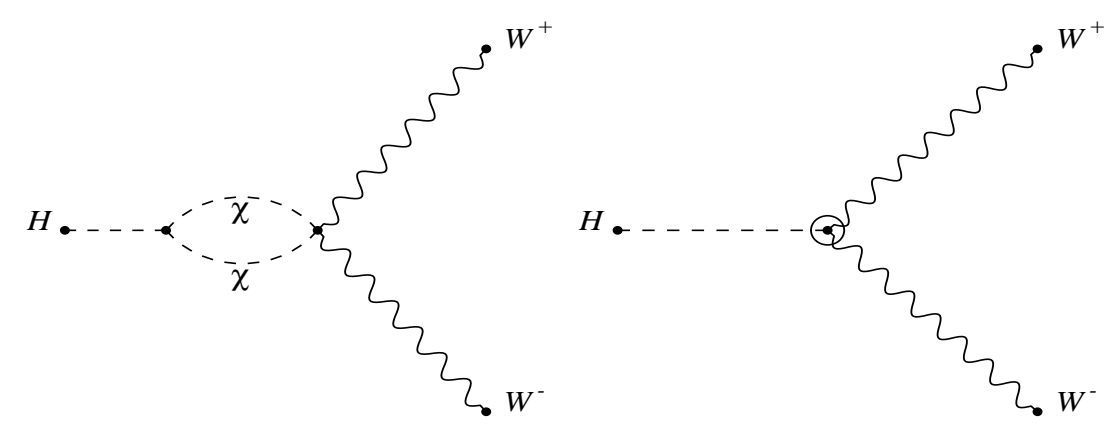

produced by GRACEFIG

imaginary part. The inclusion of the imaginary part of the wave function renormalisation is in fact just a convenient short-cut. More correctly had we taken into account the Higgs self-energy insertion (together with the counterterm) on the external leg of the Higgs ${ }^{9}$, see Fig. 13, the $\tilde{\delta}$ dependence would drop completely. In the non-linear gauge we found a few examples for other non-linear gauge-fixing parameters and for other particles besides the Higgs, like the top in $t \rightarrow b W^{+}$.

In principle, if one is only interested in corrections to the total cross section, at the one-loop level it is irrelevant whether the imaginary part of the wave function renormalisation is included or not. This is because the effect of the wave function renormalisation is an overall multiplicative factor to the amplitude. For the total cross section one only needs $\Re e\left(T_{\text {tree }} \times T_{1-\text { loop }}^{\dagger}\right)$, where $T_{\text {tree }}$ and $T_{1-\text { loop }}$ refer to the tree and one-loop amplitude respectively. Since the wave function renormalisation contribution, $Z_{p}$ for a particle $p$, to the one-loop amplitude is $Z_{p} T_{\text {tree }}$, only $\Re e Z_{p}$ would be picked up. But in applications where CP violation is an issue [99] one might need the correct gauge invariant one-loop amplitude. This effectively requires that the imaginary part of the wave function renormalisation be implemented if one is to avoid external leg insertions. This confirms that it is essential to require gauge independence at the level of the amplitude rather than in the cross section. The gauge dependence argument makes the inclusion of the absorptive part of the self-energy a necessity, although the gauge dependent part must cancel against other contributions, in all generality this should not be the case for the gauge independent part which should then be observable. For yet another aspect of the wave function renormalisation,

\footnotetext{
${ }^{9}$ Some care should be exercised for external leg insertions, such as factors of $1 / 2$ and apparent $0 / 0$ divisions. For detailed worked out examples see 98 .
} 
Figure 13: Self-energy insertion on the external leg of the Higgs (right) with its counterterm (left) for $H \rightarrow W^{+} W^{-}$.

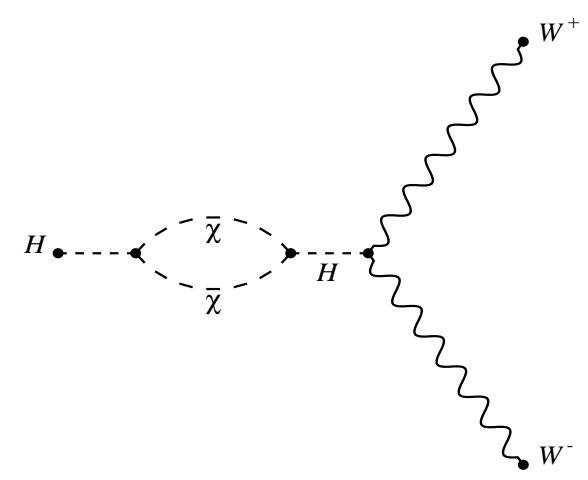

produced by GRACEFIG

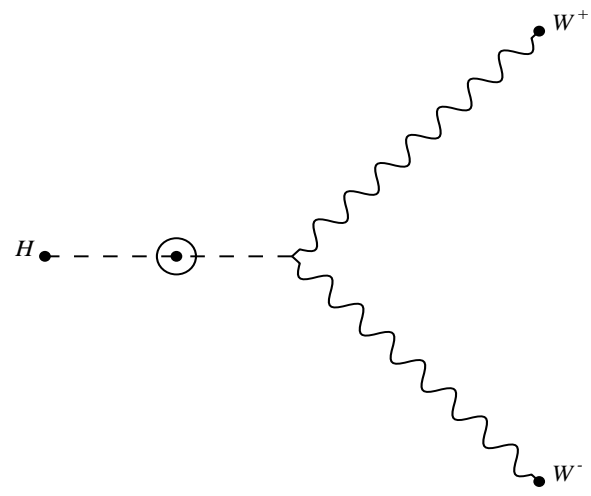

produced by GRACEFIG

see 100 .

\subsubsection{Width implementation for resonant diagrams and gauge invariance}

Since it is difficult to reconcile the concept of an asymptotic state with an unstable particle, it has been argued 101 not to consider S-matrix elements for external unstable particles. This provides an easy way out to the problem we have just discussed concerning the wave function renormalisation, especially that such an unstable particle decays into stable particles. Nonetheless the treatment of unstable particles even as internal particles poses problems. Even when calculating tree-level processes one has to regularise the propagator of an unstable particle if one is close to the resonance region. This regularisation brings in elements which are outside the order at which the perturbative calculation is being carried out. This problem is exacerbated when performing loop calculations with unstable particles. Take a tree-level matrix element where part of the contribution is due to the exchange of a massive particle which, in the following, we will take to be a gauge boson,

$$
\mathcal{M}^{0}=\frac{R(s)}{s-M^{2}}+T(s)
$$

$s$ is some invariant mass and $M$ is the mass of the particle. $R(s)$ represents the "resonant" contribution and the remainder $T$ some non resonant contribution. Although there are a few instances where $R(s)$ and $T(s)$ are separately gauge independent, in the most general case this need not be the case. $\mathcal{M}^{0}$ in Eq. 6.28 is gauge independent. The residue of the pole $R\left(M^{2}\right)$ and 
the non pole remnant $\tilde{T}(s)$, see below, are however independently gauge invariant 102, 103, 104, 105 .

$$
\mathcal{M}^{0}=\frac{R\left(M^{2}\right)}{s-M^{2}}+\overbrace{\left(\frac{R(s)-R\left(M^{2}\right)}{s-M^{2}}+T(s)\right)}^{\tilde{T}(s)} .
$$

Therefore the naive way of implementing a constant width $\Gamma$,

$$
\mathcal{M}_{\Gamma}^{0}=\frac{R(s)}{s-M^{2}+i M \Gamma}+T(s)
$$

although numerically regulating the resonant behaviour, breaks, in general, gauge invariance and can lead to disastrous results, see Ref. [106] for such an example. Often the use of a running width is made $\Gamma \rightarrow \Gamma(s)$. The latter is justified on the basis that it emerges from Dyson summation. However this summation, that moreover mixes the orders in perturbation theory, is only made on the self-energy two-point function. It therefore breaks gauge invariance since only one part of the total contribution to the amplitude is corrected. It has been found [107, for tree-level process, that in fact in most cases the running width does so much worse than the constant width. One proposal to remedy this situation while insisting on using the running width was to generalise the Dyson summation to include accompanying corrections to vertices. This scheme, the fermion pole scheme 108, 107, 109, 110, is in fact only part of the full one-loop calculation which is quite unpractical especially from the point of view of an automatic code. Moreover the scheme only takes into account fermion loops for the gauge boson propagators and is only meant as an effective means to correct tree-level predictions. There is also an effective Lagrangian approach to implement this scheme with some considerations to the unstable top quark and Higgs 111, 112.

Another scheme that is easily adapted to an automatic implementation and that can be carried beyond tree-level is the factorisation scheme. Starting with the gauge independent matrix element of Eq. 6.28 one endows the total contribution with the overall factor $\left(s-M^{2}\right) /\left(s-M^{2}+i M \Gamma\right)$, such that

$$
\mathcal{M}^{0} \rightarrow \frac{s-M^{2}}{s-M^{2}+i M \Gamma} \mathcal{M}^{0} .
$$

Although gauge invariant, this is unsatisfactory as it puts all non-resonant contributions to zero close to the resonance. The pole scheme 102, 103, 104, 105, based on an expansion around the pole offers some insight. At tree-level only the pole term is regulated by the introduction of a width, while the non-resonant gauge invariant remnants are not put to zero at the resonance. This amounts to consider

$$
\mathcal{M}_{p}^{0}=\frac{R\left(M^{2}\right)}{s-M^{2}+i M \Gamma}+\left(\frac{R(s)-R\left(M^{2}\right)}{s-M^{2}}+T(s)\right)
$$

In practice however, especially when one deals with many gauge bosons and for multi-leg processes, this procedure becomes untractable taking into account that there might be a clash 
in reconstructing the invariant $s$ from other momenta especially if kinematical cuts are to be applied [105]. A variant of the pole scheme 113] in an effective Lagrangian approach has also been advocated but its effectiveness has not been fully demonstrated for processes of practical interest.

The majority of the schemes we have reviewed so far have mainly been applied to tree-level processes although some implementations like the fermion loop scheme are attempts at including parts of the higher order corrections as are some of the effective Lagrangian approaches. It is fair to say that there is as yet no fully satisfactory solution for one-loop process. Nonetheless, especially for neutral current processes that require the introduction of a width, an automatic implementation can be performed.

Treatment of the $Z$ width in GRACE-loop: $e^{+} e^{-} \rightarrow e^{+} e^{-} H$ as an example

Figure 14: Contributing diagrams at tree-level in terms of the s-channel type, left panel, obtained from $e^{+} e^{-} \rightarrow Z H$, and the t-channel type from $Z Z$ fusion.
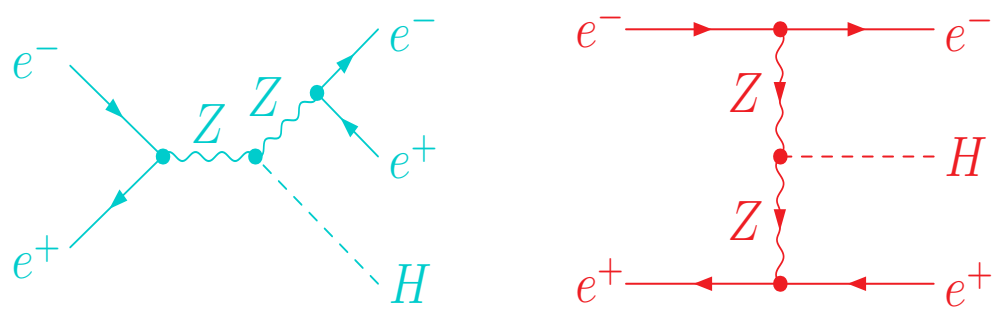

Let us take as an example the case of $e^{+} e^{-} \rightarrow e^{+} e^{-} H$ at one-loop 42. At tree-level, in the unitary gauge, the $e^{+} e^{-} \rightarrow e^{+} e^{-} H$ process is built up from an $s$-channel diagram originating from $e^{+} e^{-} \rightarrow Z H$ and a $t$-channel diagram which is a fusion type, see Fig. 14. Each type constitutes, on its own, a gauge independent process. In fact the former (neglecting lepton masses) can be defined as $e^{+} e^{-} \rightarrow \mu^{+} \mu^{-} H$. This case therefore falls in the category where $R(s)$ (s-channel here) and $T(s)$ ( $t$-channel here) of Eq. 6.28 are separately gauge invariant. In principle it is only the $Z$ coupling to the outgoing lepton, in this $s$-channel contribution, which can be resonating and thus requires a finite width. Nonetheless in our code we dress both $Z$ in the $s$-channel type diagrams with a constant $Z$ width. We apply no width to the $Z$ taking part in the $Z Z$-fusion diagrams.

To help understand our implementation at one-loop it is instructive to display a selection of some of the contributing diagrams at one-loop.

The introduction of a width is required only for the $Z$ coupling to the final electron pair. This $s$-channel contribution is much smaller than the $t$-channel contributions for which we do not endow the $Z$ propagators with a width. Building up on the implementation of the width at treelevel, we include a constant width to all $Z$ propagators not circulating in a loop for the $s$-channel type diagrams. For example we add a width to all $Z$ in graphs 349,762,4311 of Fig. 15. For those one-loop diagrams with a self-energy correction to any $Z$ propagator, represented by graph 
Figure 15: A small selection of different classes of loop diagrams contributing to $e^{+} e^{-} \rightarrow e^{+} e^{-} H$. We keep the same graph numbering as that produced by the system. Graph 4311 belongs to the corrections from self-energies, here both the virtual and counterterm contributions are generated and counted as one diagram. Graph 349 shows a vertex correction. Both graphs are considered as s-channel resonant Higgs-strahlung contributions. Graph 762 represents a box correction, it is a non resonant contribution, which can not be deduced from $e^{+} e^{-} \rightarrow Z H$, but applies also to the correction to the s-channel $e^{+} e^{-} \rightarrow \mu^{+} \mu^{-} H$. Graph 1481 is also a box correction counted as a correction to the $Z Z$ fusion. Graph 1575, Graph 1741 and Graph 1757 are fusion type corrections involving $\gamma \gamma, Z \gamma$ and $Z Z$ fusion. Graph 2607 shows a pentagon correction which also counts as an s-channel since it is induced for $e^{+} e^{-} \rightarrow \mu^{+} \mu^{-} H$. Graph 3157 on the other hand is a pentagon correction that only applies to $e^{+} e^{-} \rightarrow e^{+} e^{-} H$.
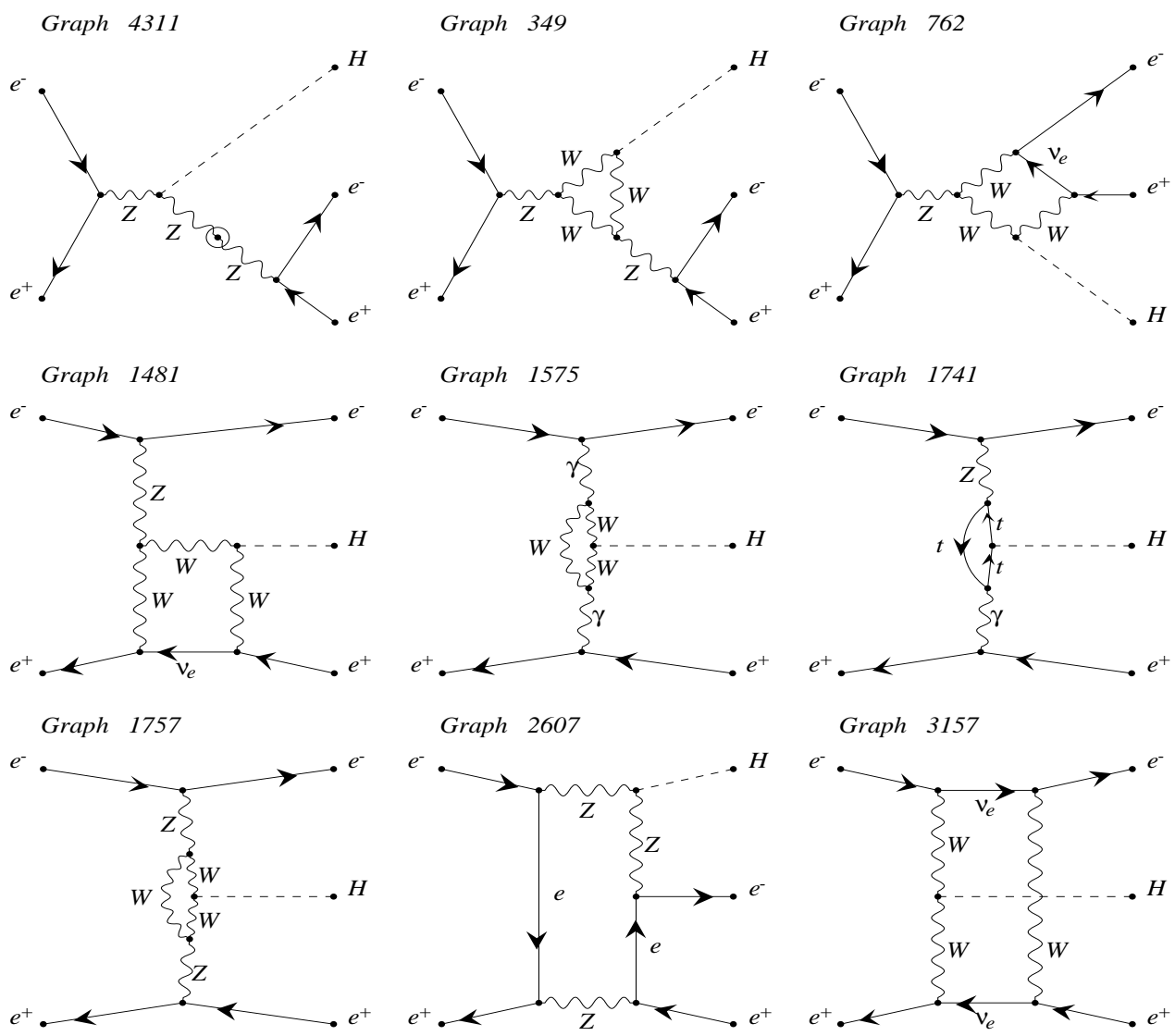

produced by GRACEFIG 
4311 in Fig. [15] we follow a procedure along the lines described in 114. We will show how this is done with a single $Z$ exchange coupling to a fermion pair of invariant mass $s_{f \bar{f}}$.

First, it is important to keep in mind that our tree-level calculation of the $s$-channel is done by supplying the width in the $Z$ propagator. Therefore it somehow also includes parts of the higher order corrections to the $Z$ self-energy which should be subtracted when performing a higher order calculation. The case at hand is as simple as consisting, at tree-level, of one single diagram. The simplest way to exhibit this subtraction is to rewrite, the zero-th order amplitude, $\mathcal{M}^{(0)}$, before inclusion of a width, in terms of what we call the tree-level (regularised) amplitude, $\widetilde{\mathcal{M}}^{\text {tree }}$

$$
\mathcal{M}^{(0)}=\frac{\mathcal{N}^{(0)}}{s_{f \bar{f}}-M_{Z}^{2}}=\overbrace{\frac{\mathcal{N}^{(0)}}{s_{f \bar{f}}-M_{Z}^{2}+i \Gamma_{Z} M_{Z}}}^{\widetilde{\mathcal{M}}^{\text {ree }}}\left(1+\frac{i\left(\Gamma_{Z}^{0}+\Delta \Gamma_{Z}\right) M_{Z}}{s_{f \bar{f}}-M_{Z}^{2}}\right), \quad \Delta \Gamma_{Z}=\Gamma_{Z}-\Gamma_{Z}^{0} \cdot
$$

The $\Gamma_{Z}^{0}$ contribution will be combined with the one-loop correction while $\Delta \Gamma_{Z}$ will be counted as being beyond one-loop.

At the one-loop level, before the summation à la Dyson and the inclusion of any "hard" width, the amplitude is gauge-invariant and can be decomposed as

$$
\begin{aligned}
\mathcal{M}^{(1)} & =\frac{\mathcal{N}^{(0)}}{s_{f \bar{f}}-M_{Z}^{2}} \frac{\tilde{\Pi}_{T}^{Z Z}\left(s_{f \bar{f}}\right)}{s_{f \bar{f}}-M_{Z}^{2}}+\frac{A_{Z \gamma} \frac{\tilde{\Pi}_{T}^{Z \gamma}\left(s_{f \bar{f}}\right)}{s_{f \bar{f}}}}{s_{f \bar{f}}-M_{Z}^{2}}+\frac{R_{Z}}{s_{f \bar{f}}-M_{Z}^{2}}+C \\
& =\frac{1}{s_{f \bar{f}}-M_{Z}^{2}}\left\{\mathcal{N}^{(0)} \frac{\tilde{\Pi}_{T}^{Z Z}\left(s_{f \bar{f}}\right)}{s_{f \bar{f}}-M_{Z}^{2}}+\left(A_{Z \gamma} \frac{\tilde{\Pi}_{T}^{Z \gamma}\left(s_{f \bar{f}}\right)}{s_{f \bar{f}}}+R_{Z}\right)+\left(s_{f \bar{f}}-M_{Z}^{2}\right) C\right\}
\end{aligned}
$$

The different contributions in $\mathcal{M}^{(1)}$ are the following. The first term proportional to the treelevel contribution is due to the renormalised transverse part of the $Z$ self-energy correction $\tilde{\Pi}_{T}^{Z Z}$, including counterterms. Such a transition is shown in Graph 4311 of Fig. 15. The term proportional to $A_{Z \gamma} \frac{\tilde{\Pi}_{T}^{Z \gamma}\left(s_{f \bar{f}}\right)}{s_{f \bar{f}}}$ comes from the renormalised transverse part of the $Z-\gamma$ self-energy, $\tilde{\Pi}_{T}^{Z \gamma}$, with the photon attaching to the final fermion (this type is absent for neutrinos in $e^{+} e^{-} \rightarrow$ $\left.\nu_{e} \bar{\nu}_{e} H\right)$. The $R_{Z}$ terms combine one-loop corrections which nevertheless still exhibit a $Z$ exchange that couples to the final fermions and hence these types of diagrams can be resonant, an example is Graph 349 of Fig. 15. We can write $R_{Z}=Z_{Z H}+V_{Z}^{f}$, where $Z_{Z H}$ corresponds to the part containing the correction to $e^{+} e^{-} \rightarrow Z^{\star} H$, while $V_{Z}^{f}$ contains the corrections to the final $Z_{f \bar{f}}$ vertex. $Z_{Z H}\left(s_{f \bar{f}}=M_{Z}^{2}\right)$ corresponds to $e^{+} e^{-} \rightarrow Z H$ and is gauge invariant at the pole. The term $C$ contains all the rest which are apparently non-resonant ${ }^{10}$, an example here is Graph 762 of Fig. 15. Both $\mathcal{M}^{(1)}$ and $\mathcal{M}^{(0)}$ are gauge invariant.

Our procedure, in effects, amounts to first regularising the overall propagator in Eq. 6.34 by the implementation of a constant $Z$ width and then combining the renormalised $Z$ self-energy

\footnotetext{
${ }^{10}$ Strictly speaking we, here, deal only with the pure weak corrections. In the infrared limit some of the QED diagrams can be resonant and require a $Z$ width even in a loop. This is discussed in Ref. 42 .
} 
part in Eq. 6.34 with the $\Gamma_{Z}^{0}$ part of Eq. 6.33 Since our on-shell renormalisation procedure is such that $\operatorname{Re} \tilde{\Pi}_{T}^{Z Z}\left(M_{Z}^{2}\right)=0$ and since $\Gamma_{Z}^{0}=-\operatorname{Im} \Pi_{T}^{Z Z}\left(M_{Z}^{2}\right)$, see [115], our prescription is to write

$$
\begin{aligned}
\mathcal{M}^{(0)}+\mathcal{M}^{(1)} & \rightarrow \overbrace{\frac{\mathcal{N}^{(0)}}{s_{f \bar{f}}-M_{Z}^{2}+i \Gamma_{Z} M_{Z}}+\overbrace{\frac{1}{s_{f \bar{f}}-M_{Z}^{2}+i \Gamma_{Z} M_{Z}} \widetilde{\mathcal{N}}^{1}}^{\widetilde{\mathcal{M}}^{\text {tree }}}}^{\widetilde{\mathcal{M}}^{\text {looop }}} \\
\widetilde{\mathcal{N}}^{1} & =\mathcal{N}^{(0)} \frac{\left(\tilde{\Pi}_{T}^{Z Z}\left(s_{f \bar{f}}\right)-\tilde{\Pi}_{T}^{Z Z}\left(M_{Z}^{2}\right)\right)}{s_{f \bar{f}}-M_{Z}^{2}}+\left(A_{Z \gamma} \frac{\tilde{\Pi}_{T}^{Z \gamma}\left(s_{f \bar{f}}\right)}{s_{f \bar{f}}}+R_{Z}\right) \\
& +\left(s_{f \bar{f}}-M_{Z}^{2}\right) C .
\end{aligned}
$$

The above prescription is nothing else but the factorisation procedure avoiding double counting. It is gauge invariant but puts the non-resonant terms to zero on resonance. In practice in the automatic code, we supply a constant $Z$ width to all $Z$ not circulating in a loop and by treating the one-loop $Z Z$ self-energy contribution as in Eq. 6.35. Up to terms of order $\mathcal{O}\left(\Gamma_{Z} \alpha\right)$ this is equivalent to Eq. 6.35. In particular the contribution of the $C$ term does not vanish on resonance, since its overall factor is unity rather than the factor $\left(s_{f \bar{f}}-M_{Z}^{2}\right) /\left(s_{f \bar{f}}-M_{Z}^{2}+i \Gamma_{Z} M_{Z}\right)$ that would be present in the original factorisation prescription.

The complex mass scheme

This scheme stems from the very simple observation that the parameter $M^{2}$ in Eq. 6.28 could be taken from the outset as having an imaginary part. This imaginary part should be included consistently even when it enters through couplings and mixings as is the case for the electroweak mixing defined in Eq. 5.14 through the ratio of the mass of the (unstable) $W$ and $Z$. All the algebraic relations will therefore be maintained in this analytic continuation, in particular gauge invariance is not broken. Identifying the imaginary part with the implementation of a width, at tree-level this scheme has been used in [116]. This simple analytical continuation is rather easily implemented in an automatic code for the calculation of Feynman diagrams. The roots of this idea 117. pre-date its first practical usage in a tree-level calculation and, in fact, emerged from considerations at the loop level. The suggestion is that when splitting the real bare parameters, as is done in Eq. 6.1, one may well take the renormalised and the counterterm parameters to be both complex and similarly for the fields and the wave function renormalisation as suggested already by our discussion in section 6.5.1. The only subtle problem now is that the renormalised Lagrangian is not Hermitian. This poses then the problem of perturbative unitarity and how one defines the Cutksoky cutting rules [118. Barring this issue, a full implementation of this scheme has been worked out at one-loop and first applied to $e^{+} e^{-} \rightarrow 4 f$ [51]. Its implementation in an automatic code at one-loop is rather straightforward since it amounts to define the counterterms for the parameters of Eq. 6.1 and wave function renormalisation of Eq. 6.2 by including the imaginary parts of the two-point functions, whereas the usual on-shell scheme is based on taking only the real parts at the appropriate renormalisation scale. The use of complex masses as we have stressed needs to be carried out consistently by analytical continuation. This means that even the corresponding masses that enter the loop diagrams need to be complex. This calls for 
the extension of the loop function, which we treat in the next chapter (Chapter 7), to include complex mass arguments. This automatically regularises some of the infrared resonant loop integrals, a result that had been also arrived at by the direct inclusion of the width in the loop so that factorisation of the infrared factor be maintained [114. For more details about this scheme applied at one-loop see Ref. [51. As it can be inferred by this presentation this scheme lends itself to an easy implementation in a code for the automatic calculations of Feynman diagrams.

\section{Evaluation of the loop integrals}

Figure 16: General structure of the $N$-point loop integral. $l$ is the loop momentum, $M_{i}$ are the masses of the particles circulating in the loop. $p_{i}$ are the external momenta. $s_{2}$ is a combination of external momenta, see Eq.7.2.

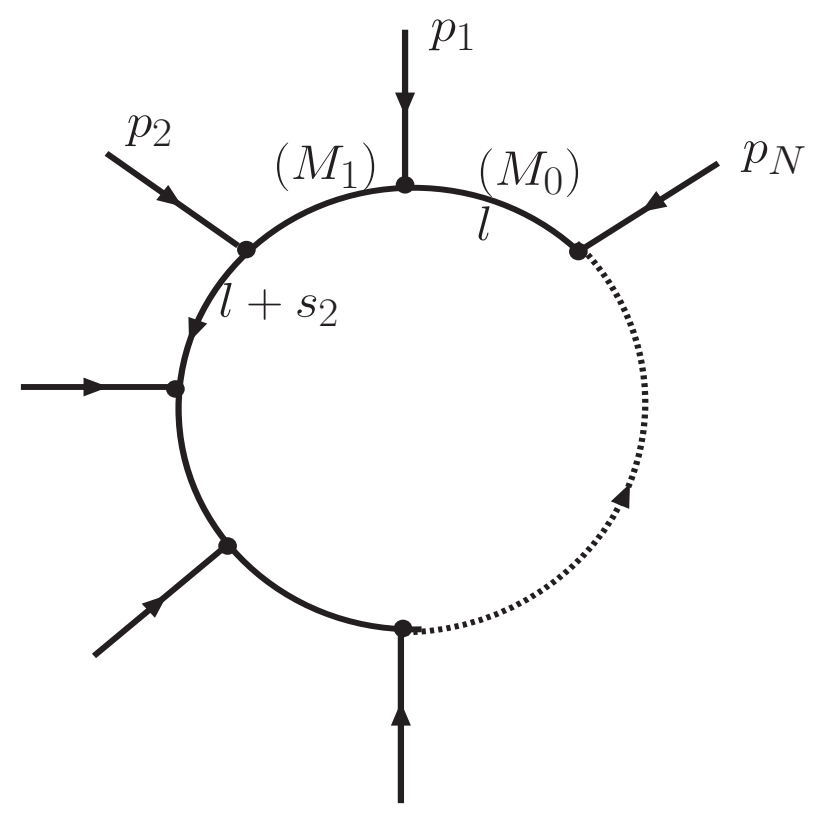

The evaluation of the loop integrals is one of the most important ingredients of a loop calculation. This is also one of the most time consuming especially as the number of external legs increases. A generic loop integral involving $N$ external particles is depicted in Fig. 16. The tensor integral of rank $M$ corresponding to a $N$-point graph that we encounter in the general non-linear gauge but with Feynman parameters $\xi=1$ are such that $M \leq N$. The object in question writes in DR as

$$
\underbrace{(N) \cdots \rho}_{M}=\int \frac{d^{n} l}{(2 \pi)^{n}} \frac{l_{\mu} l_{\nu} \cdots l_{\rho}}{D_{0} D_{1} \cdots D_{N-1}}, \quad M \leq N
$$


where

$$
D_{i}=\left(l+s_{i}\right)^{2}-M_{i}^{2}, \quad s_{i}=\sum_{j=1}^{i} p_{j}, \quad s_{0}=0 .
$$

$M_{i}$ are the internal masses, $p_{i}$ the incoming momenta and $l$ the loop momentum.

The $N$-point scalar integrals correspond to $M=0$. All higher rank tensors for a $N$-point function, $M \geq 1$, can be deduced recursively from the knowledge of the $N$-point (and lower) scalar integrals. In GRACE-loop all tensor reductions of two, three and four-point functions are performed by solving a system of equations obtained by taking derivatives with respect to the Feynman parameters. All higher orders parametric integrals corresponding to the tensor integrals can then be recursively derived from the scalar integral, as will be described below. It is important to stress that this reduction is different to what is usually done through the Passarino-Veltman [119] or the Brown-Feynman [120] reductions. It is also different from the approach of Bern, Dixon and Kosower 121 who exploit differentiation of the scalar integral with respect to a set of kinematical variables.

Although the present review mainly describes the methods of one-loop calculations for up to $2 \rightarrow 2$ processes, where only $N \leq 4$ loop integrals are needed, we will describe briefly the very recent development in the calculation of one-loop processes with 5 external legs and in one instance 6 legs and in particular how the $N=5,6$-point functions are treated in GRACE-loop. For $N \geq 5$ all integrals can be reduced to $N=4$-point functions.

Since the computation of the scalar integrals, especially for $N \leq 4$ is central let us first describe their implementation in GRACE-loop. Let us note that, in the intermediate stage of the symbolic calculation dealing with loop integrals (in $n$-dimension), we extract the regulator constant $C_{U V}$ defined in Eq. 4.9. We treat $C_{U V}$ as a parameter in the subsequent (numerical) stages. We regularise any infrared divergence by giving the photon a fictitious mass, $\lambda$. By default we set this at $\lambda=10^{-15} \mathrm{GeV}$.

\subsection{Scalar integrals for $N \leq 4$}

The two-point integrals are implemented using simple analytical formulae and evaluated numerically. This allows to achieve a quite high precision. The scalar 3-point function and all but the infrared divergent 4-point scalar functions are evaluated through a call to the FF package 122 . Although the FF package has been extensively used and checked by many authors, we have also tested its accuracy and implementation in GRACE-loop by comparing its results against our own numerical approach to loop integrals 123 .

For the infrared four-point function, see Fig. 17, we supply our own optimised routines. A purely numerical approach would lead to instabilities and would prevent a complete and satisfactory cancellation of infrared divergences between these loop functions and the infrared factors from the real soft bremsstrahlung part. Luckily some rather simple analytical results have been derived in this case 114, 124. These can be further simplified when the box involves quite separate mass scales as often occurs in $e^{+} e^{-}$(smallness of the electron mass). In the 
Figure 17: The left panel shows the general configuration of the infrared four-point functions of the scattering of incoming particles with masses $m_{1}, m_{2}$ to particles with masses $m_{3}, m_{4}$. $s, t$ are the usual Mandelstam variables. The right panel shows some examples that need to be treated carefully. In the first example, the $Z$ can be close to the resonance.
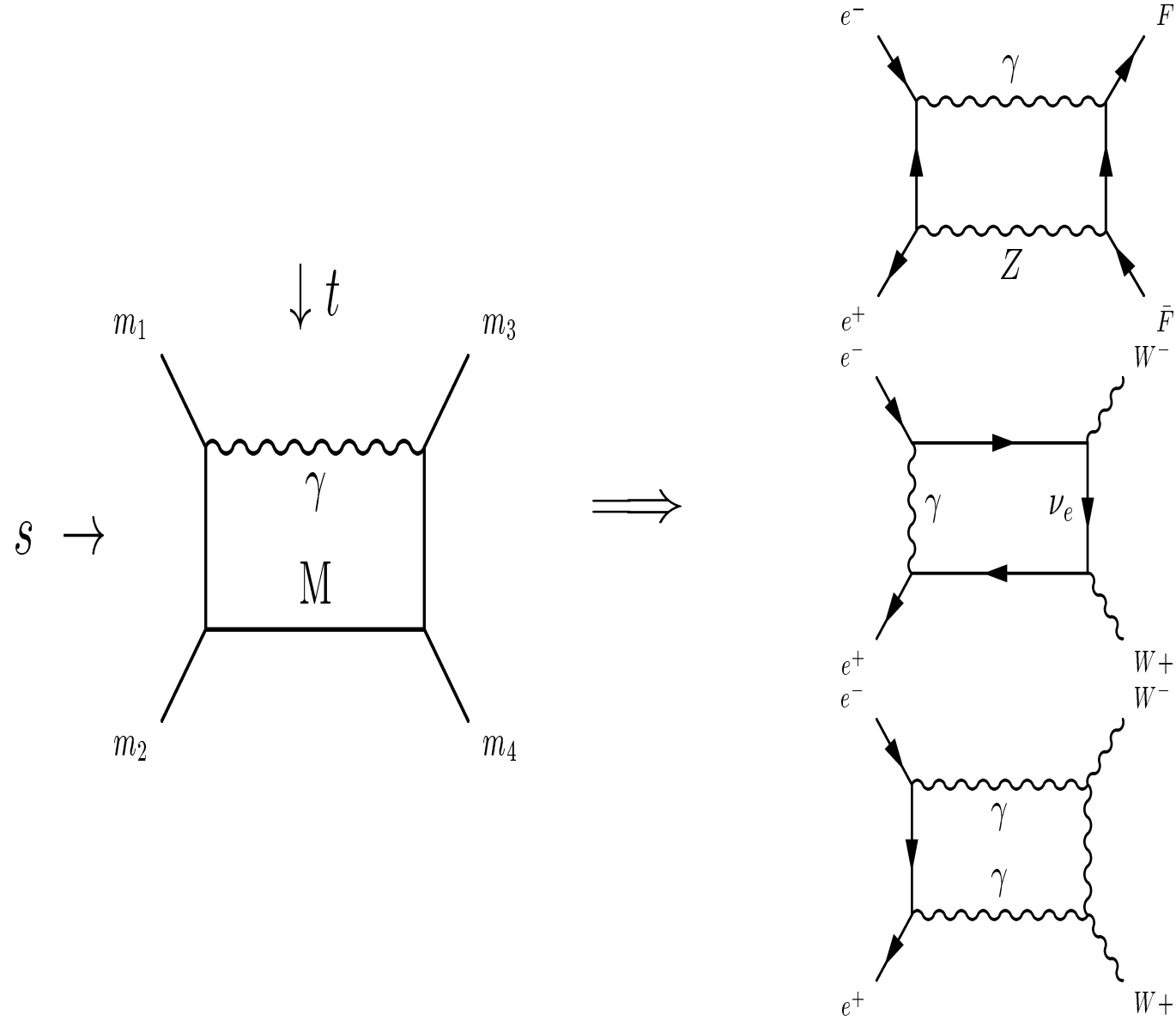

example $e^{+} e^{-} \rightarrow F \bar{F}$ shown in Fig. 17, close to the $Z$ resonance, $s \simeq M_{Z}^{2}$, one needs to take into account the width of the $Z$ circulating in the loop. This implementation ensures that, even close to the resonance, the infrared divergent part exactly cancels against the soft bremsstrahlung correction. The calculation of some of these photonic boxes is detailed in [114] and [124].

\subsection{Reduction of the tensor integrals for $N \leq 4$}

The tensor integral of rank $M$ corresponding to a $N$-point graph is defined in Eq. [7.1. The use of Feynman's parameterisation combines all propagators such that

$$
\frac{1}{D_{0} D_{1} \cdots D_{N-1}}=\Gamma(N) \int[d x] \frac{1}{\left(D_{1} x_{1}+D_{2} x_{2}+\cdots D_{0}\left(1-\sum_{i=1}^{N-1} x_{i}\right)\right)^{N}}
$$




$$
\int[d x]=\int_{0}^{1} d x_{1} \int_{0}^{1-x_{1}} d x_{2} \cdots \int_{0}^{1-\sum_{i=1}^{N-2} x_{i}} d x_{N-1} .
$$

Because the integrals are regulated, we first deal with the loop momenta, before handling the integration over the parametric variables and write

$$
\begin{aligned}
& \underbrace{T_{\mu \nu \cdots \rho}^{(N)} \cdots \rho}_{M}=\Gamma(N) \int[d x] \underbrace{\mathcal{T}_{\mu \nu \cdots \rho}^{(N)}}_{M}, \quad \text { with } \\
& \underbrace{\mathcal{T}_{\mu \nu \cdots \rho}^{(N)} \cdots \rho}_{M}=\int \frac{d^{n} l}{(2 \pi)^{n}} \frac{l_{\mu} l_{\nu} \cdots l_{\rho}}{\left(l^{2}-2 l . P\left(x_{i}\right)-M^{2}\left(x_{i}\right)\right)^{N}}, \quad M \leq N
\end{aligned}
$$

Integration over the loop momenta $l$ is done trivially. We may write a compact formula that applies up to the boxes. Introducing

$$
\begin{aligned}
\Delta & =\sum_{i, j=1}^{N-1} Q_{i j} x_{i} x_{j}+\sum_{i=1}^{N-1} L_{i} x_{i}+\Delta_{0}, \quad Q_{i j}=s_{i} . s_{j}, \quad L_{i}=-s_{i}^{2}+\left(M_{i}^{2}-M_{0}^{2}\right), \\
\Delta_{0} & =M_{0}^{2}, \quad P=-\sum_{i=1}^{N-1} s_{i} x_{i}
\end{aligned}
$$

we can write

$$
\begin{aligned}
\mathcal{T}^{(N)} & =\widetilde{\mathcal{T}}^{(N)} \Gamma(N-n / 2) \quad \text { with } \quad \widetilde{\mathcal{T}}^{(N)}=\frac{(-1)^{N} i \pi^{n / 2}}{(2 \pi)^{n} \Gamma(N)} \Delta^{-(N-n / 2)}, \\
\mathcal{T}_{\mu}^{(N)} & =\mathcal{T}^{(N)} P_{\mu}, \\
\mathcal{T}_{\mu \nu}^{(N)} & =\widetilde{\mathcal{T}}^{(N)}\left(\Gamma(N-n / 2) P_{\mu} P_{\nu}-\frac{1}{2} g_{\mu \nu} \Delta \Gamma(N-1-n / 2)\right) \\
\mathcal{T}_{\mu \nu \rho}^{(N)} & =\widetilde{\mathcal{T}}^{(N)}\left(\Gamma(N-n / 2) P_{\mu} P_{\nu} P_{\rho}-\frac{\Delta}{2}\left(g_{\mu \nu} P_{\rho}+g_{\mu \rho} P_{\nu}+g_{\nu \rho} P_{\mu}\right) \Gamma(N-1-n / 2)\right) \\
\mathcal{T}_{\mu \nu \rho \sigma}^{(N)} & =\widetilde{\mathcal{T}}^{(N)}\left(\Gamma(N-n / 2) P_{\mu} P_{\nu} P_{\rho} P_{\sigma}+\frac{\Delta^{2}}{4}\left(g_{\mu \nu} g_{\rho \sigma}+g_{\mu \rho} g_{\nu \sigma}+g_{\mu \sigma} g_{\nu \rho}\right) \Gamma(N-2-n / 2)\right. \\
& \left.-\frac{\Delta}{2}\left(g_{\mu \nu} P_{\rho} P_{\sigma}+g_{\mu \rho} P_{\nu} P_{\sigma}+g_{\mu \sigma} P_{\nu} P_{\rho}+g_{\nu \rho} P_{\mu} P_{\sigma}+g_{\nu \sigma} P_{\mu} P_{\rho}+g_{\rho \sigma} P_{\mu} P_{\nu}\right) \Gamma(N-1-n / 2)\right)
\end{aligned}
$$

It now rests to integrate over the Feynman parameters contained in the momenta $P=P\left(\left\{x_{i}\right\}\right)$. We show how this is done for the box $(N=4)$ and triangle $(N=3)$ integrals. As pointed out earlier the case $N=2$ is straightforward and is implemented analytically, some examples are given in Appendix G. The problem now turns into finding solutions for the parametric integrals

$$
\underbrace{I_{i \cdots k}^{(N)}}_{M}=\int[d x] \frac{x_{i} \cdots x_{k}}{\Delta^{(N-2)}} \text { and }
$$




$$
J_{i ; \alpha}^{(N)}=\int[d x] x_{i}^{\alpha} \log \Delta \quad \alpha=0,1 .
$$

solely in terms of the scalar integral $I^{(N)}=\int[d x] \Delta^{-(N-2)}$ (for which we use the FF package[122]). The appearance of the integrals $J_{i ; \alpha}^{(N)}$ stems from expanding Eq. [7.6 around $n=4-2 \epsilon$ and originates from the $\epsilon$ independent terms in $\epsilon\left(1 / \epsilon+\mathcal{O}\left(\epsilon^{0,1}\right)\right)$. In fact one only needs $J^{(4)}=J_{i ; 0}^{(4)}$

and $J_{i ;(0,1)}^{(3)}$. All these integrals are derived recursively. The integral in Eq. 7.7 will be referred to as the parametric integral of rank- $M$ for the $N$-point function. It will then be expressed in terms of lower rank tensors and lower $N$ integrals.

\subsection{Reduction of the higher rank parametric box integrals}

Let us first show how the tensor box integrals are implemented. Note that one needs 15 different integrals for the rank- 4 box, 10 for the rank-3, 6 for the rank- 2 and 3 for the rank- 1 . The trick is to use the fact that

$$
\begin{gathered}
\int[d x] \partial_{i}\left(\frac{x_{k}^{\alpha} x_{l}^{\beta} x_{m}^{\gamma}}{\Delta}\right), \\
\text { with } \quad \partial_{i}=\frac{\partial}{\partial x_{i}}, \quad 1 \leq \alpha+\beta+\gamma=M \leq N-1
\end{gathered}
$$

is a surface term that can be derived from the parametric integrals of the triangle. On the other hand expanding the partial derivative generates parametric integrals of order $M+1$, beside parametric integrals of rank $M-1$ and rank $M$. To wit,

$$
\begin{aligned}
\partial_{i}\left(\frac{x_{k}^{\alpha} x_{l}^{\beta} x_{m}^{\gamma}}{\Delta}\right)= & -\frac{x_{k}^{\alpha} x_{l}^{\beta} x_{m}^{\gamma}}{\Delta^{2}}\left(L_{i}+2 \sum_{j} Q_{i j} x_{j}\right)+\frac{1}{\Delta^{2}}\left(\Delta_{0}+\sum_{j} L_{j} x_{j}+\sum_{j n} Q_{j n} x_{j} x_{n}\right) \times \\
& \left(\alpha x_{k}^{\alpha-1} x_{l}^{\beta} x_{m}^{\gamma} \delta_{k i}+\beta x_{l}^{\beta-1} x_{k}^{\alpha} x_{m}^{\gamma} \delta_{l i}+\gamma x_{m}^{\gamma-1} x_{k}^{\alpha} x_{l}^{\beta} \delta_{m i}\right)
\end{aligned}
$$

The term on the left-hand side can be trivially integrated and can be expressed in terms of the triangle integral of rank $M \leq 3$. The terms proportional to $L_{i}$ on the right-hand side are box integrals of rank $M$ whereas the term proportional to $\Delta_{0}$ corresponds to boxes of rank $M-1$. We combine all these terms into $C_{i ; j k l}$, where the first index $i$ shows that a derivative has been applied on the index $i$. The terms proportional to $Q_{i j}$ are boxes of rank $M+1$ that we want to derive. In particular, to generate the highest rank integrals for the box $M=4$, we apply Eq. 7.10 with $\alpha=\beta=\gamma=1,(M=3)$. This amounts to solving a system of equations for the integrals $I_{i j k l}^{(4)}$,

$$
\begin{aligned}
C_{i ; k l m} & =-2 \sum_{j} Q_{i j} I_{j k l m}^{(4)}+\sum_{j n} Q_{j n}\left(\delta_{k i} I_{j n l m}^{(4)}+\delta_{l i} I_{j n k m}^{(4)}+\delta_{m i} I_{j n k l}^{(4)}\right) \\
I_{i j k l}^{(4)} & =\int[d x] \frac{x_{i} x_{j} x_{k} x_{l}}{\Delta^{2}}
\end{aligned}
$$


One can thus solve for a system of equations for the higher rank parametric integrals of order $M+1$ in terms of (previously derived) integrals of rank $M-1$ and $M$ for the box and rank $M$ for the triangle. One drawback of this approach is that one ends up with a larger set of equations than needed to solve the system, especially as the rank of the parametric integral increases. This however also shows that one can in principle carry consistency checks. To pick up a system of linearly independent equations we first construct

$$
\sum_{i} C_{i ; i k k}=3 \sum_{j n} Q_{j n} I_{j n k k}^{(4)}
$$

in order to form the set ${ }^{11}$

$$
\tilde{C}_{i ; k k k}=-\frac{1}{2}\left(C_{i ; k k k}-\delta_{k i} \sum_{i} C_{i ; i k k}\right)=\sum_{j} Q_{i j} I_{j k k k}^{(4)} .
$$

For the highest rank, $M=4$, this provides 3 independent sets (one for each value of $k$ ) each consisting of three independent integrals $I_{j k k k}^{(4)}$ (for $j=1,2,3$ ). Therefore one only deals with 3 simple $3 \times 3$ matrices which help solve 9 out of 15 integrals. We may refer to this set as the diagonal integrals. The remaining integrals are provided by the set of the 6 independent equations $C_{i ; j k k}$ where $i, j, k$ are all different from each other,

$$
C_{i ; j k k}=-2 \sum_{n} Q_{i n} I_{n j k k}^{(4)}, \quad i \neq j \neq k
$$

It is obvious that the same trick applies to solving $I_{j k k}^{(4)}$ and provides 9 out of the 10 independent integrals. The remaining integral in this case is provided by any $C_{i ; j k}$ where all indices are different. We also apply Eq. 7.13 to the set $I_{j k}^{(4)}$ and obtain $\tilde{C}_{i ; k}=\sum_{j} Q_{i j} I_{j k}^{(4)}$.

This method shows that to solve for the 15 independent integrals of rank-4 one does not deal with a $15 \times 15$ matrix. Rather the previous formulation shows that this splits into simplified $3 \oplus 3 \oplus 3 \oplus 6$ systems of equations. The $6 \times 6$ matrix is also easy to deal with since each row consists of only 2 non-zero elements. For the rank-3 integrals the system of 10 equations decomposes into $3 \oplus 3 \oplus 3 \oplus 1$, while for the rank-2, the system of 6 equations decomposes into $3 \oplus 2 \oplus 1$.

${ }^{11}$ This could have also been arrived at more directly had we used

$$
\sum_{j} x_{j} \partial_{j} \Delta=2 \Delta-2 \Delta_{0}-\sum_{j} L_{j} x_{j}
$$

This enables to re-express the second terms on the right-hand side of Eq. 17.10 involving the Kronecker symbols for the case $\alpha=\beta=\gamma=1$, as lower rank terms and triangle integrals. Namely we can write

$$
3 \frac{x_{l} x_{m}}{\Delta}=-2 \frac{\Delta_{0}}{\Delta^{2}}+\sum_{j}\left\{\partial_{j}\left(\frac{x_{j} x_{l} x_{m}}{\Delta}\right)-L_{j}\left(\frac{x_{j} x_{l} x_{m}}{\Delta^{2}}\right)\right\} .
$$




\section{4 $\log (\Delta)$ terms for the box and triangle}

To extract $J^{(4)}$ for the box and most of the results for the reduction of the higher rank integrals for the triangle, we start by giving a general representation for the logarithm. Take $\Delta_{N}$ where $N$ is to just remind us that it comes from a $N$-point function. We can write

$$
x_{i}^{\alpha} \log \Delta_{N}=\frac{1}{N+\alpha-1} \sum_{j=1}^{N-1}\left\{\partial_{j}\left(x_{i}^{\alpha} x_{j} \log \Delta_{N}\right)-x_{i}^{\alpha} x_{j} \partial_{j}\left(\log \Delta_{N}\right)\right\}
$$

Specific formulae needed for the boxes and triangles are

$$
\begin{aligned}
x_{i} \log \Delta & =\frac{1}{3} \sum_{j=1}^{2}\left\{\partial_{j}\left(x_{i} x_{j} \log \Delta\right)-x_{i}+\frac{x_{i}\left(L_{j} x_{j}+\Delta_{0}\right)}{\Delta}\right\}, N=3, \alpha=1 \\
\log \Delta & =\frac{1}{2} \sum_{j=1}^{2}\left\{\partial_{j}\left(x_{j} \log \Delta\right)-1+\frac{L_{j} x_{j}+\Delta_{0}}{\Delta}\right\}, N=3, \alpha=0 \\
\log \Delta & =\frac{1}{3} \sum_{j=1}^{3}\left\{\partial_{j}\left(x_{j} \log \Delta\right)-\frac{2}{3}+\frac{\Delta\left(L_{j} x_{j}+2 / 3 \Delta_{0}\right)}{\Delta^{2}}\right\}, N=4, \alpha=0
\end{aligned}
$$

Eq. 17.18 shows that $J^{(4)}$ can be expressed in terms of the "lower" integrals $J_{i ; 1}^{(3)}$ and $I_{M=0,1,2,3}^{(4)}$. In turn, all $J_{i ;(0,1)}^{(3)}$ are expressed in terms of two-point functions and the integrals $I_{M=0,1,2}^{(3)}$.

\subsection{Reduction of the higher rank parametric integrals for the triangle}

To generate the triangle $I_{M=1,2,3}^{(3)}$, in analogy with Eq. [7.10, we use

$$
\partial_{i}\left(x_{k}^{\alpha} x_{l}^{\beta} \log \Delta\right)
$$

For example, for $M=3$ exploiting Eq. [7.17 we get

$$
\begin{aligned}
\partial_{i}\left(x_{k} x_{l} \log \Delta\right) & =\frac{x_{k} x_{l}}{\Delta}\left(L_{i}+2 \sum_{j} Q_{i j} x_{j}\right) \\
& +\frac{1}{3}\left\{\delta_{k i} \sum_{j}\left(\partial_{j}\left(x_{l} x_{j} \log \Delta\right)-x_{l}\left(1-\frac{L_{j} x_{j}+\Delta_{0}}{\Delta}\right)\right)+(k \leftrightarrow l)\right\} .
\end{aligned}
$$

All terms with $L_{i}, \Delta_{0}$ or partial derivatives are lower order terms (either in $N$ or $M$ ). Grouping all these as $C_{i ; k l}$ leads to the master equation 


$$
C_{i ; k l}=\sum_{j} Q_{i j} I_{j k l}^{(3)}
$$

Following the same strategy as with the box, we choose the set $C_{i ; k k}$ which furnishes 2 "orthogonal" systems of 2 equations each. Therefore instead of handling a $4 \times 4$ matrix we only deal with simple $2 \times 2$ matrices. Similar results are obtained for $I_{j k}^{(3)}$. The three needed integrals are arrived at by first solving for a reduced system of only two independent integrals and then deriving the third from a single equation.

Finally for $M=1$, one solves a system with a $2 \times 2$ matrix. Note that the solution of all these equations involves the determinant of the same $2 \times 2$ matrix, namely $Q_{i j}$.

Note also that the system of equations as described here leads to analytic solutions in terms of the scalar integrals. In GRACE-1oop we implement these analytical solutions.

\subsection{Reduction of 5- and 6-point integrals}

Five point functions are calculated as linear combinations of four point functions 125, 126]. Variants and new techniques have also been worked out very recently 40, 127, 128. We will describe the methods that have been implemented in GRACE-loop. The reduction takes advantage of the fact that for $N>4$ not all the external momenta are linearly independent. For $N=5$, the set of vectors $\left\{s_{i}\right\}$ with $i=1, \cdots 4$, see Eq. 7.2, forms an independent basis of 4 -vectors, which allows to expand any 4-momentum, particularly the loop momentum $l$ as

$$
l^{\mu}=\sum_{i, j=1}^{4}\left(Q^{-1}\right)_{i j}\left(l \cdot s_{i}\right) s_{j}^{\mu}
$$

where the $4 \times 4$ matrix $Q_{i j}$ is defined as in Eq. 7.5 with $i, j=1, \cdots 4$.

From Eq.(17.22) we express $l^{2}$ as

$$
l^{2}=\sum_{i, j=1}^{4}\left(Q^{-1}\right)_{i j}\left(l \cdot s_{i}\right)\left(l \cdot s_{j}\right)
$$

which helps define an identity between the denominators of the propagators in the 5-point function. Using the same notation as in Eqs. [7.2 [7.5] we rewrite

$$
D_{0}+M_{0}^{2}=\frac{1}{2} \sum_{i, j=1}^{4}\left(Q^{-1}\right)_{i j}\left(D_{i}-D_{0}+L_{i}\right)\left(l \cdot s_{j}\right),
$$

to arrive at the identity

$$
4 M_{0}^{2}-\sum_{i, j=1}^{4}\left(Q^{-1}\right)_{i j}\left(L_{i}\right)\left(D_{j}-D_{0}+L_{j}\right)=-4 D_{0}+2 \sum_{i, j=1}^{4}\left(Q^{-1}\right)_{i j}\left(D_{i}-D_{0}\right)\left(l \cdot s_{j}\right) .
$$


This demonstrates that a 5 -point function with a numerator of the form $N(l)=l^{\mu_{1}} \cdots l^{\mu_{k}}$ is expressed as a sum of five box integrals,

$$
\begin{aligned}
& \left(4 M_{0}^{2}-\sum_{i, j=1}^{4}\left(Q^{-1}\right)_{i j} L_{i} L_{j}\right) \int \frac{\mathrm{d}^{4} l}{(2 \pi)^{4} i} \frac{N(l)}{\mathcal{D}_{\mathbf{5}}}= \\
& \int \frac{\mathrm{d}^{4} l}{(2 \pi)^{4} i} N(l)\left(-\frac{4 D_{0}}{\mathcal{D}_{\mathbf{5}}}+\sum_{i, j=1}^{4}\left(Q^{-1}\right)_{i j} L_{i} \frac{D_{j}-D_{0}}{\mathcal{D}_{\mathbf{5}}}+2 \sum_{i, j=1}^{4}\left(Q^{-1}\right)_{i j} \frac{\left(D_{i}-D_{0}\right)\left(l \cdot s_{j}\right)}{\mathcal{D}_{\mathbf{5}}}\right),
\end{aligned}
$$

where

$$
\mathcal{D}_{\mathbf{5}}=D_{0} \prod_{i=1}^{4} D_{i} .
$$

Putting $N(l)=1$ one gets the reduction formula of the 5-point scalar integral to a sum of four-point integrals. This method has been applied to $e^{+} e^{-} \rightarrow \nu_{e} \bar{\nu}_{e} H$ [2] and $e^{+} e^{-} \rightarrow t \bar{t} H$ [45] where the highest rank tensor of the pentagon is $M=2$. We will refer to this technique, for short, as the scalar-derived reduction.

Although this technique can be directly applied to a 5-point integral of any rank $M \leq N=5$, we should note that the presence of the term $l \cdot s_{j}$ in Eq. 7.26 raises the rank of the integral by one unit. This causes a superficial UV divergence for $M \geq 3$. For $M=4$ the reduction requires the evaluation of $M=5$ box diagrams, a case that is not covered by our reduction formulae of the tensor boxes, see section [7.3. Furthermore when this formula is used to get the matrix elements in a symbolic way, the resultant FORTRAN code usually becomes very lengthy. We have developed another algorithm for the reduction of higher rank tensors which we first applied to $e^{+} e^{-} \rightarrow Z H H$ [3]. We apply the identity Eq.(7.22) to the numerator $N(l)$. We have

$$
\begin{aligned}
N(l) & =l^{\mu_{1}} l^{\mu_{2}} \cdots l^{\mu_{k}}=\sum_{i, j=1}^{4}\left(Q^{-1}\right)_{i j}\left(l \cdot s_{i}\right) s_{j}^{\mu_{1}} l^{\mu_{2}} \cdots l^{\mu_{k}}, \\
& =\frac{1}{2} \sum_{i, j=1}^{4}\left(Q^{-1}\right)_{i j}\left(D_{i}-D_{0}+L_{i}\right) s_{j}^{\mu_{1}} l^{\mu_{2}} \cdots l^{\mu_{k}} .
\end{aligned}
$$

Then

$$
\begin{aligned}
\int \frac{\mathrm{d}^{4} l}{(2 \pi)^{4} i} \frac{N(l)}{\mathcal{D}_{\mathbf{5}}} & =\frac{1}{2} \sum_{i, j=1}^{4}\left(Q^{-1}\right)_{i j} s_{j}^{\mu_{1}} \int \frac{\mathrm{d}^{4} l}{(2 \pi)^{4} i} \frac{\left(D_{i}-D_{0}\right) l^{\mu_{2}} \cdots l^{\mu_{k}}}{\mathcal{D}_{\mathbf{5}}} \\
& +\frac{1}{2} \sum_{i, j=1}^{4}\left(Q^{-1}\right)_{i j} L_{i} s_{j}^{\mu_{1}} \int \frac{\mathrm{d}^{4} l}{(2 \pi)^{4} i} \frac{l^{\mu_{2}} \cdots l^{\mu_{k}}}{\mathcal{D}_{\mathbf{5}}} .
\end{aligned}
$$

On the right-hand-side the rank of the numerator is lowered by one unit, though there still remains a sum of 5-point functions. This reduction can be repeated until one is left with a scalar 5-point function and box integrals. We will, for short, refer to this method as the vectorderived reduction. An advantage of this method is that the final expression in FORTRAN code is about ten times shorter than that obtained by the previous technique. 
Let us also very briefly describe how the 6-point functions are implemented in GRACE-1oop [52]. In this case, we first express $s_{5}$ in terms of the set of four linearly independent vectors $s_{i}, i=$ $1, \cdots, 4$ and construct the product

$$
l \cdot s_{5}=\sum_{i, j=1}^{4}\left(Q^{-1}\right)_{i j}\left(s_{5} \cdot s_{i}\right)\left(l \cdot s_{j}\right)
$$

hence

$$
-L_{5}+\sum_{i, j=1}^{4}\left(Q^{-1}\right)_{i j}\left(s_{5} \cdot s_{i}\right) L_{j}=D_{5}-D_{0}-\sum_{i, j=1}^{4}\left(Q^{-1}\right)_{i j}\left(s_{5} \cdot s_{i}\right)\left(D_{j}-D_{0}\right) .
$$

Combining with

$$
\mathcal{D}_{6}=D_{0} \prod_{i=1}^{5} D_{i}
$$

we find

$$
\begin{aligned}
\left(-L_{5}+\sum_{i, j=1}^{4}\left(Q^{-1}\right)_{i j}\left(s_{5} \cdot s_{i}\right) L_{j}\right) \int \frac{\mathrm{d}^{4} l}{(2 \pi)^{4} i} \frac{N(l)}{\mathcal{D}_{6}} \\
\quad=\int \frac{\mathrm{d}^{4} l}{(2 \pi)^{4} i} \frac{\left(D_{5}-D_{0}\right) N(l)}{\mathcal{D}_{6}}-\sum_{i, j=1}^{4}\left(Q^{-1}\right)_{i j}\left(s_{5} \cdot s_{i}\right) \int \frac{\mathrm{d}^{4} l}{(2 \pi)^{4} i} \frac{\left(D_{j}-D_{0}\right) N(l)}{\mathcal{D}_{6}} .
\end{aligned}
$$

This is the standard reduction of a general 6-point function to a sum of 5-point integrals. Moreover for further reduction of the 5-point integrals we use a combination of both the scalarderived Eq. 7.22 and vector-derived Eq. 7.28 reductions for the loop tensor $N(l)$ of rank- $M$ to arrive at a reduction which in a compact form writes as

$$
\overbrace{N(l)}^{M}=\sum_{i, j=1}^{4} \overbrace{R_{i, j}(l)}^{M-2} D_{i} D_{j}+\sum_{i} \overbrace{S_{i}}^{M=0} D_{i}+\overbrace{T_{6}}^{M=0}
$$

The first term of the right-hand side is tensor of rank $M-2$ which corresponds to box diagrams. The next two-terms correspond to scalar 5-point and 6-point function for which we use the algorithm of the scalar-derived reduction and the one based on Eq. 7.33 for the 6-point function. Once the reduction of the 5-point and 6-point tensor function have been brought down to box integrals with a lower tensor rank, we use the algorithm developed for $N \leq 4$ as described in detail in section 7.1 and 7.2. Infrared resonant 5-point and 6-point functions that require the introduction of a width in the loop integrals require more care, see [52].

In actual computations the matrix element for 5- and 6-point diagrams is most time consuming, since as we have just seen the reduction algorithms are quite involved and go through various steps. For example the percentage of $\mathrm{CPU}$ time needed to calculate $N$-point diagrams 
is summarized in table 2 for a $2 \rightarrow 3$ and $2 \rightarrow 4$ process. One can see for instance, in the case of $e^{+} e^{-} \rightarrow \nu_{e} \bar{\nu}_{e} H H$ that almost as many as 2000 three-point loop integrals require $8 \%$ of the CPU time whereas the 746 -point integrals require $67 \%$ of the CPU time.

Table 2: Percentage of CPU time among various $N$-point loops. The number of diagrams in each class of $N$-point diagram is shown in parenthesis. "Others" stands for two-point functions and counterterms.

\begin{tabular}{|c|c|c|c|c|c|}
\hline process & 6-point & 5-point & 4-point & 3-point & others \\
\hline$e^{+} e^{-} \rightarrow e^{+} e^{-} H$ & - & $33 \%$ & $11 \%$ & $47 \%$ & $9 \%$ \\
& - & $(20)$ & $(44)$ & $(348)$ & $(98)$ \\
\hline$e^{+} e^{-} \rightarrow \nu_{e} \bar{\nu}_{e} H H$ & $67 \%$ & $13 \%$ & $10 \%$ & $8 \%$ & $2 \%$ \\
& $(74)$ & $(218)$ & $(734)$ & $(1804)$ & $(586)$ \\
\hline
\end{tabular}

As we need a lot of computer power, it is essential to develop software engineering techniques in order to reduce the execution time. In GRACE we have developed a parallelised version that exploits message passing libraries, such as PVM or MPI 129. Concerning the loop calculation, it is efficient to distribute calculations of each Feynman diagram among many CPUs because it is hard to create a single executable file from too long source codes.

Another technique must be vectorisation which can make CPU much shorter. Thanks to the recent rapid development of microprocessors and their easy availability even for the common public one should think of building or adapting codes to run as vectorised codes that can be quite effective. Once one has enough memory/cache, the vectorisation of the amplitudes could lead to a much more powerful tool. It must be said however, that vectorisation of existing codes is not always straightforward or even possible. For example, GRACE relies on the package FF 122] for the evaluation of the one-loop scalar integrals (boxes, triangles). Because of its structure that employs too many if ... then ... else ... end if statements, the FF package is not fully vectorised in the current GRACE system.

\subsection{New techniques for the loop integrals}

The reduction formalism that we have outlined both for the reduction of the $N$-point functions with $N \geq 4$ to the lower $N$-point functions and the tensorial reduction, even for $N \leq 4$, to the scalar integrals involves implicitly the inverse of the determinant of the matrix $Q_{i j}$, the Gram determinant. This is most apparent in our formulation of section [7.6, see for example Eq. [7.28 which is expressed in terms of $Q^{-1}$. For kinematical configurations where $\operatorname{Det} Q$ is very small or vanishes this can lead to severe numerical instabilities. It must be said that these exceptional configurations are, in a Monte Carlo sampling, hardly met. Moreover, the numerical instability around these singularities can be cured if one reverts to quadruple, or higher, precision. It has been shown [130. how this solution can be optimised with a dedicated FORTRAN library for multiprecision operations such that it does not require much CPU time while keeping the benefit 
of exploiting the same standard reduction formalism.

For $N \leq 4$ and for $\operatorname{Det} Q=0$, there also exist very efficient algorithms 131, 132, 133 that are amenable to an automatic computer implementation. Expansions about vanishing Gram determinants are also possible 132, 134, 128. Recently there has been a lot of activity to improve this aspect of the loop calculation by finding new, improved and efficient algorithms for the loop integrals especially to avoid this problem. One approach is, instead of reducing the system to the master set of the scalar integrals with $N \leq 4$, to use other bases for the master integrals which can include a tensorial integral for example, therefore avoiding the appearance of Gram determinants before a numerical calculation is performed. These approaches 127, 128, 40, 133, 135. combine both an algebraic reduction with an efficient numerical implementation. Other approaches are, to a very large extent, essentially based on a numerical computation of all the loop integrals 136, 137, 138, 139, 140, 141. One example is based on the contour deformation of the multi-dimensional parameter integrals 140] and may be implemented in GRACE-1oop. On the other hand, for loops with internal massless particles as would be the case for applications to QCD, some powerful algebraic methods of the loop integrals are being derived [142, 143]. Let

us also mention that most of the methods extract the ultraviolet and infrared divergences, so that the set of basis integrals is amenable to an efficient implementation.

It should be kept in mind that the majority of the new techniques have not been implemented as fully working codes yet nor has their robustness been tested in practical calculations, especially as concerns multi-leg processes. Apart from [40, 128 which, in fact, is an extension of the standard reduction that has been applied successfully to $e^{+} e^{-} \rightarrow 4 f$ [51], it remains to be seen how the other new techniques perform when handling the complete one-loop calculation of a physical process, of interest for the LHC or the LC.

\section{Tests on the loop calculation}

The results of the calculations are checked by performing three kinds of tests. This concerns the ultraviolet and infrared finiteness as well as the gauge-parameter independence. These tests are performed at the level of the differential cross section before any phase space integration is performed for several points in phase space. These tests points are chosen at random. Usually for these tests one keeps all diagrams involving the couplings of the Goldstones to the light fermions, such as $\chi_{3} e^{+} e^{-}$. For these tests to be passed one works in quadruple precision. After these tests have been passed one can switch off these very small couplings, involving the scalars and the light fermions, when calculating the total (integrated) cross section and hence speeding up the computation time. Results of these tests on a selection of the 26 processes for the $2 \rightarrow 2$ reactions displayed in Table. 3 are made available at this web location[144]. This list involves both purely vector bosons scattering, heavy as well as massless fermions scattering into gauge bosons as well as a few processes involving the Higgs. Therefore, as we will see, all the ingredients that enter the calculation of radiative corrections in the $\mathcal{S M}$ are covered by this list. 


\subsection{Ultraviolet and infrared finiteness checks}

\subsubsection{Ultraviolet finiteness}

We first check the ultraviolet finiteness of the results. This test applies to the whole set of the virtual one-loop diagrams. The ultraviolet finiteness test gives a result that is stable over 30 digits when one varies the dimensional regularisation parameter $C_{U V}$ defined in Eq. 4.9, This parameter is kept in the code as a free parameter. This parameter could then be set to 0 in further computations once the finiteness test, or $C_{U V}$ independence test, is passed. When conducting this test we regularise any infrared divergence by giving the photon a fictitious mass that we fix at $\lambda=10^{-15} \mathrm{GeV}$. The finiteness test is carried out for a random series of the gauge fixing parameters that include the linear gauge as a special case.

\subsubsection{Infrared finiteness and calculation of the soft-bremsstrahlung factor}

When discussing the calculation and implementation of the loop integrals, some diagrams involving a photon exchange require special treatment. These diagrams lead to an infrared divergence caused by the fact that the photon is massless so that its energy could vanish. These infrared divergences in the case of the photon, either in QED or in the electroweak theory, can be regulated by giving the photon a small mass $\lambda$. As known the dependence in this fictitious mass cancels against the one contained in the soft bremsstrahlung [145] and do not hinder the renormalisation procedure. For a textbook introduction see for instance [80 or [146]. In the non-Abelian case where there is no smooth mass limit, an example being QCD, this regularisation of the infrared divergence by giving the gauge boson a mass would fail[147]. In this case one can revert to dimensional regularisation [148. In GRACE, for the electroweak radiative corrections we use the simple trick of the fictitious photon mass to regulate the infrared divergences.

The second test that we perform relates to the infrared finiteness by checking that when the virtual loop correction and bremsstrahlung contributions are added there is no dependence on the fictitious photon mass $\lambda$. We indeed find results that are stable over 23 digits, or better, when varying $\lambda$.

The soft bremsstrahlung part consists of the tree-level process with an additional photon of very small energy, $E_{\gamma}<k_{c}$, and requires the introduction of the photon mass regulator, $\lambda$. The hard photon radiation with $E_{\gamma}>k_{c}$ is regular and will be discussed in section [8.3. The soft photon contribution is implemented in the system following an analytical result based on factorisation and which can be generalised to any process. The bremsstrahlung differential cross section factorises as

$$
d \sigma_{\text {soft }}\left(\lambda, E_{\gamma}<k_{c}\right)=d \sigma_{0} \times \delta_{\text {soft }}\left(\lambda, E_{\gamma}<k_{c}\right) .
$$

$k_{c}$ is assumed sufficiently small so that the tree-level $d \sigma_{0}$ does not change rapidly when the soft photon is emitted. In some cases, for instance around a resonance, special care must be exercised, see for example 114. The factor $\delta_{\text {soft }}$ is completely determined from the classical (convection) current of a charged particle and does not involve the spin connection. Therefore this factor is universal and only depends on the charge $Q_{i}$ and momentum $p_{i}$ of the particles of the tree-level process, 


$$
\delta_{\text {soft }}=-e^{2} \int_{|k|<k_{c}} \frac{d^{3} k}{2 E_{\gamma}(2 \pi)^{3}} \sum_{i j} \varepsilon_{i} \varepsilon_{i} Q_{i} Q_{j} \frac{p_{i} \cdot p_{j}}{\left(k \cdot p_{i}\right)\left(k \cdot p_{j}\right)}=\sum_{i j} R_{i j}, \quad E_{\gamma}=\sqrt{k^{2}+\lambda^{2}}
$$

where $\varepsilon_{i}= \pm 1$ depending on whether the particle is incoming $(+1)$ or outgoing $(-1)$. Very general expressions for $R_{i j}$ have been derived most elegantly in [149]. Let us here just recall a few special cases and refer the reader to [114] for more details. For instance, for the diagonal term $R_{i i}$ from a charged particle with $|Q|=1$ of momentum $p=(E, \vec{p}), p^{2}=m^{2}$ and $P=|\vec{p}|$, one gets the very simple result

$$
R_{i i}=-e^{2} \int_{|k|<k_{c}} \frac{d^{3} k}{2 E_{\gamma}(2 \pi)^{3}} \frac{m^{2}}{(k \cdot p)^{2}}=-\frac{\alpha}{\pi}\left\{\ln \left(\frac{2 k_{c}}{\lambda}\right)+\frac{E}{P} \ln \left(\frac{m}{E+P}\right)\right\} .
$$

Another quite useful result is the contribution, $R_{\text {pair }}$, from a pair of particle-antiparticle of mass $m$ and charge \pm 1 in their centre-of-mass system with total energy $\sqrt{s}$. The radiator function writes, with $\beta=\sqrt{1-4 m^{2} / s}$

$$
\begin{aligned}
R_{\text {pair }}=\frac{2 \alpha}{\pi}\left\{\left(\frac{s-2 m^{2}}{s \beta} \ln \left(\frac{1+\beta}{1-\beta}\right)-1\right) \ln \left(\frac{2 k_{c}}{\lambda}\right)+\frac{1}{2 \beta} \ln \left(\frac{1+\beta}{1-\beta}\right)\right. \\
\left.-\frac{s-2 m^{2}}{2 s \beta}\left(\operatorname{Li}_{2}\left(\frac{2 \beta}{1+\beta}\right)-\operatorname{Li}_{2}\left(\frac{-2 \beta}{1-\beta}\right)\right)\right\},
\end{aligned}
$$

and

$$
\operatorname{Li}_{2}(z)=-\int_{0}^{z} d t \frac{\ln (1-t)}{t}
$$

is the Spence function. This factor would represent the initial state bremsstrahlung part in $e^{+} e^{-}$processes and is usually written $\left(\right.$for $s \gg m_{e}^{2}$ ) as

$$
R_{\text {pair }}^{e^{+} e^{-}}=\frac{2 \alpha}{\pi}\left\{\left(\ln \left(\frac{s}{m_{e}^{2}}\right)-1\right) \ln \left(\frac{2 k_{c}}{\lambda}\right)-\frac{1}{4} \ln ^{2}\left(\frac{s}{m_{e}^{2}}\right)+\frac{1}{2} \ln \left(\frac{s}{m_{e}^{2}}\right)-\frac{\pi^{2}}{6}\right\} .
$$

The same factor in Eq. 8.4 can be used as the bremsstrahlung contribution for $\gamma \gamma \rightarrow W^{+} W^{-}$ $\left(m \rightarrow M_{W}\right)$.

\subsection{Gauge-parameter independence checks}

For this check we set the value of the ultraviolet parameter $C_{U V}$ to some fixed value. To tame the infrared divergence contained in the virtual corrections we give the photon a fictitious mass $\lambda=10^{-15} \mathrm{GeV}$. Moreover we also set all widths to zero so that no extra gauge breaking due to the introduction of a width is generated. We thus choose a non-singular point in phase space, away from any resonance, for this check on the differential cross section. 
Table 3: Accuracy measured by the number of digits for the gauge-parameter checks on the 26 processes for all five gauge parameters. The numbers that appear in the last five columns represent the number of digits which are stable when varying the corresponding gauge parameter. An empty entry means that the process does not depend on the gauge parameter. Only one parameter is varied at a time here. We also show the number of diagrams both at tree-level and at the one-loop level. The number of diagrams depends on the choice of the gauge parameter, for examples in some gauges some vertices are absent. The number of diagrams that we list corresponds to the gauge which leads to the maximum number of diagrams.

\begin{tabular}{|c||c||c|c|c|c|c|}
\hline processes & \# of graphs(Loop $\times$ Tree $)$ & $\tilde{\alpha}$ & $\tilde{\beta}$ & $\tilde{\delta}$ & $\tilde{\epsilon}$ & $\tilde{\kappa}$ \\
\hline$\nu_{e} \bar{\nu}_{e} \rightarrow \nu_{e} \bar{\nu}_{e}$ & $46 \times 2$ & - & 30 & - & - & - \\
$e^{+} e^{-} \rightarrow \nu_{e} \overline{\nu_{e}}$ & $112 \times 3$ & 31 & 31 & 31 & - & 32 \\
$e^{+} e^{-} \rightarrow t \bar{t}$ & $150 \times 4$ & 32 & 31 & 31 & 31 & 31 \\
$e^{+} e^{-} \rightarrow e^{+} e^{-}$ & $288 \times 4$ & 32 & 30 & 30 & 31 & 31 \\
$e^{+} e^{-} \rightarrow W^{+} W^{-}$ & $334 \times 4$ & 27 & 27 & 30 & 31 & - \\
$e^{+} e^{-} \rightarrow Z^{0} Z^{0}$ & $336 \times 3$ & 33 & 29 & 31 & 31 & - \\
$e^{+} e^{-} \rightarrow H^{0} Z^{0}$ & $341 \times 3$ & 30 & 30 & 31 & 31 & 30 \\
$\mu \overline{\nu_{\mu}} \rightarrow W^{-} \gamma$ & $162 \times 3$ & 27 & 27 & 28 & - & 28 \\
$\mu \overline{\nu_{\mu}} \rightarrow W^{-} Z^{0}$ & $213 \times 4$ & 31 & 29 & 30 & - & 30 \\
$\mu \overline{\nu_{\mu}} \rightarrow W^{-} H^{0}$ & $196 \times 3$ & 30 & 29 & 31 & 31 & 31 \\
$t \bar{b} \rightarrow W^{+} \gamma$ & $239 \times 4$ & 22 & 25 & 29 & - & 29 \\
$t \bar{b} \rightarrow W^{+} Z^{0}$ & $284 \times 4$ & 31 & 22 & 31 & - & 32 \\
$t \bar{b} \rightarrow W^{+} H^{0}$ & $285 \times 4$ & 29 & 28 & 21 & 26 & 30 \\
$\gamma \gamma \rightarrow t \bar{t}$ & $267 \times 2$ & 24 & 34 & 30 & - & - \\
$Z^{0} Z^{0} \rightarrow t \bar{t}$ & $338 \times 3$ & 30 & 29 & 31 & 31 & - \\
$W^{+} W^{-} \rightarrow t \bar{t}$ & $354 \times 4$ & 30 & 26 & 31 & 31 & - \\
$Z^{0} H^{0} \rightarrow t \bar{t}$ & $355 \times 4$ & 30 & 28 & 29 & 29 & 31 \\
$\gamma \gamma \rightarrow W^{+} W^{-}$ & $619 \times 5$ & 22 & 24 & 32 & - & 31 \\
$Z^{0} Z^{0} \rightarrow Z^{0} Z^{0}$ & $657 \times 3$ & - & 24 & 31 & 31 & - \\
$Z^{0} \gamma \rightarrow W^{+} W^{-}$ & $680 \times 5$ & 28 & 28 & 31 & - & 31 \\
$Z^{0} W^{-} \rightarrow Z^{0} W^{-}$ & $840 \times 6$ & 26 & 24 & 29 & 30 & 29 \\
$W^{+} W^{-} \rightarrow W^{+} W^{-}$ & $925 \times 7$ & 27 & 26 & 30 & 31 & - \\
$Z^{0} H^{0} \rightarrow W^{+} W^{-}$ & $823 \times 5$ & 29 & 25 & 29 & 26 & 31 \\
$Z^{0} H^{0} \rightarrow Z^{0} H^{0}$ & $830 \times 6$ & - & 23 & 24 & 20 & 31 \\
$H^{0} W^{-} \rightarrow H^{0} W^{-}$ & $827 \times 6$ & 29 & 23 & 22 & 23 & 30 \\
$H^{0} H^{0} \rightarrow H^{0} H^{0}$ & $805 \times 4$ & - & 29 & 27 & - \\
\hline
\end{tabular}


For each process we verify that it does not depend on any of the five non-linear gauge parameter of the set $\zeta=(\tilde{\alpha}, \tilde{\beta}, \tilde{\delta}, \tilde{\kappa}, \tilde{\epsilon})$. Let us remind the reader that we always work with $\xi_{W}=\xi_{Z}=\xi_{A}=1$. The use of five parameters is not redundant as often these different parameters check complementary sets of diagrams. For example the parameter $\tilde{\beta}$ is involved in all diagrams containing the gauge $W W Z$ coupling and their Goldstone counterpart, whereas $\tilde{\alpha}$ checks $W W \gamma$ and $\tilde{\delta}$ is implicitly present in $W W H$. For each parameter of the set, the first check is made while freezing all other four parameters to 0 .

In a second check we give, in turn, each of the remaining 4 parameters a non-zero value (we usually take the values $(2,3,4,5)$ for this set) so that we also check vertices and diagrams that involve cross terms (like $\tilde{\alpha} \times \tilde{\delta}$ ). In principle checking for 2 or 3 values of the gauge parameter should be convincing enough. We in fact go one step further and perform a comprehensive gaugeparameter independence test. To achieve this we generate for each non-linear gauge parameter $\zeta_{i}$ of the set $\zeta$, the values of the loop correction to the total differential cross section as well as the individual contribution of each one-loop diagram $g, \mathrm{~d} \sigma_{g}$ for a sequence of values for $\zeta_{i}$, while freezing the other parameters to a fixed value, not necessarily zero. The one-loop diagram contribution from each loop graph $g$ to the fully differential cross section, is defined as

$$
\mathrm{d} \sigma_{g} \equiv \mathrm{d} \sigma_{g}(\zeta)=\Re e\left(T_{g}^{\text {loop }} \cdot \mathcal{T}^{\text {tree } \dagger}\right)
$$

$\mathcal{T}^{\text {tree }}$ is the tree-level amplitude summed over all tree-diagrams. Therefore the tree-level amplitude does not depend on any gauge parameter. Note that in many processes, some individual tree diagrams do depend on a gauge parameter, however after summing over all tree-level diagrams, the gauge-parameter independence at tree-level for any process is exact within machine precision. $T_{g}^{\text {loop }}$ is the one-loop amplitude contribution of a one-loop diagram $g$. It is not difficult to see, from the structure of the Feynman rules of the non-linear gauge, that for each $2 \rightarrow 2$ process the differential cross section is a polynomial of (at most) fourth degree in the gauge parameter. Therefore the contribution $\mathrm{d} \sigma_{g}$ of diagram $g$ to the one-loop differential cross section may be written as

$$
\mathrm{d} \sigma_{g}(\zeta)=\mathrm{d} \sigma_{g}^{(0)}+\zeta \mathrm{d} \sigma_{g}^{(1)}+\zeta^{2} \mathrm{~d} \sigma_{g}^{(2)}+\zeta^{3} \mathrm{~d} \sigma_{g}^{(3)}+\zeta^{4} \mathrm{~d} \sigma_{g}^{(4)}
$$

We have therefore chosen the sequence of the five values $\zeta=0, \pm 1, \pm 2$. For each contribution $\mathrm{d} \sigma_{g}$, it is a straightforward matter, given the values of $\mathrm{d} \sigma_{g}$ for the five input $\zeta=0, \pm 1, \pm 2$, to reconstruct $d \sigma_{g}^{(0,1,2,3,4)}$. For each set of parameters we automatically pick up all those diagrams that involve a dependence on the gauge parameter. The number of diagrams in this set depends on the parameter chosen. Different parameters involve different (often) complementary sets. In some cases a very large number of diagrams is involved. An example is $Z W^{+} \rightarrow Z W^{+}$with $\tilde{\beta} \neq 0, \tilde{\kappa}=1$ where the set involves 601 one-loop diagrams out of a total of 840 . We then numerically verify that the (physical) differential cross section is independent of $\zeta$

$$
\mathrm{d} \sigma=\sum_{g} \mathrm{~d} \sigma_{g}=\sum_{g} \mathrm{~d} \sigma_{g}^{(0)}
$$


Table 4: Non-linear gauge parameter checks on $\tilde{\alpha}$ (all other parameters set to zero), for the differential cross section $W^{+} W^{-} \rightarrow W^{+} W^{-}$. For details see text.

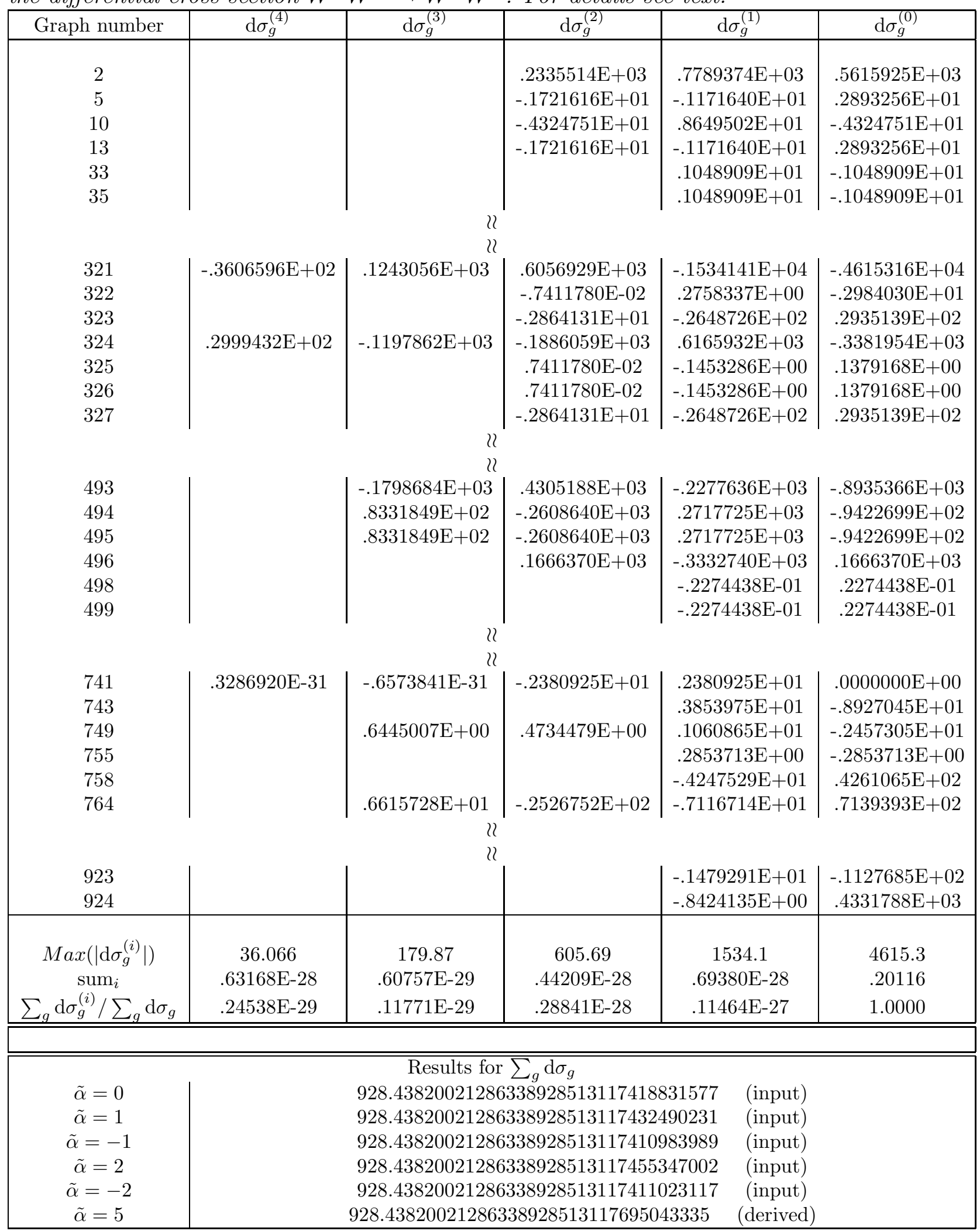


and therefore that

$$
\operatorname{sum}_{i}=\frac{\sum_{g} \mathrm{~d} \sigma_{g}^{(i)}}{\operatorname{Max}\left(\left|\mathrm{d} \sigma_{g}^{(i)}\right|\right)}=0 \quad, \quad i=1,2,3,4 .
$$

As summarised in Table 3, we find a precision of at least 21 digits on all $\mathrm{sum}_{i}$ for all the checks we have done. We usually get a much better precision when the number of diagrams involved in the check is smaller.

To appreciate how this level of accuracy is arrived at after summing on all diagrams, we show here, see Table 4, in some detail the result for $W^{+} W^{-} \rightarrow W^{+} W^{-}$for the check on the $\tilde{\alpha}$ gauge-parameter independence (all other parameters set to zero). This is extracted from the web-page where we have made these checks public 144. This process involves some 925 one-loop diagrams (and 7 at tree-level). Even for this particular example it is not possible to list all the entries of the table (that is the numerical contributions for all the diagrams) since they would not fit into a single page (the check on $\tilde{\alpha}$ involves some 336 diagrams), thus the skip (22) on some of the data. For each graph, labelled by its graph number in Table 4. we give all $\mathrm{d} \sigma_{g}^{(i)}$. We see that although individual contributions can be of the order of $10^{2}$, when summed up they give a total of the order of $10^{-27}$. We also show, at the bottom of the table, $\sum_{g} \mathrm{~d} \sigma_{g}$ for the input values $\tilde{\alpha}=0, \pm 1, \pm 2$ and compare these results to the result obtained by setting $\tilde{\alpha}=5$ in Eq. 8.8 after $d \sigma_{g}^{(0,1,2,3,4)}$ have been reconstructed. In this example concerning $\tilde{\alpha}$ we have set the values of the other gauge parameters, $\tilde{\beta}, \tilde{\delta}, \tilde{\kappa}, \tilde{\epsilon}$ to zero. We have also made a similar test on $\tilde{\alpha}$ allowing all other parameters non-zero. The same tests done on $\tilde{\alpha}$ are in turn made for all other parameters. These tests are made on 26 processes. More information on the check concerning $W^{+} W^{-} \rightarrow W^{+} W^{-}$and all those listed in Table 3 are to be found at [144.

One more note concerning the checks on the non-linear gauge parameter compared to a check one would do through the Feynman gauge parameter $\xi_{W, A, Z}$ in the usual linear gauge. Having more parameters that clearly affect different sectors differently helps in detecting any possible bug. Within the linear gauge, the usual gauge-parameter dependence is not a polynomial, it also involves $\log \xi$ and other functions of $\xi$. It is therefore almost impossible to fit the exact $\xi$ dependence of each graph. Moreover as pointed out earlier one needs to prepare new libraries for handling (very) high rank tensor integrals that are not necessary in the Feynman gauges.

\subsection{Inclusion of hard bremsstrahlung, $k_{c}$ stability}

A complete $\mathcal{O}(\alpha)$ correction necessitates the inclusion of the contribution from hard photon bremsstrahlung. Although this is a tree-level process, in most cases the total cross section can not be derived analytically. There is no difficulty in computing the matrix elements. In GRACE this is done automatically keeping all particle masses. The integration over phase space can get tricky in many cases. In fact in most cases of interest like 
for $e^{+} e^{-}$processes some care must be exercised. The reason is that though there is no infrared problem one still needs to very carefully control the $k_{c}$ dependence and also the collinear mass singularity. This $k_{c}$ dependence when combined with the one in the soft bremsstrahlung part (based on a analytical implementation), see section 8.1.2, should cancel leaving no dependence on the cut-off $k_{c}$. This would constitute another test on the $\mathcal{O}(\alpha)$ calculation of GRACE. The collinear mass singularity is most acute when the mass of the charged particle is very small compared to the typical energy scale of the problem, as in $e^{+} e^{-}$at high energies. All these problems are due to the integration over the propagators encountered in Eq. 8.2. For example, take the emission from the positron with momentum, $p$. This propagator is defined from

$$
k \cdot p=E_{\gamma}\left(E-P \cos \theta_{\gamma}\right)=E_{\gamma} P\left[\frac{m_{e}^{2}}{P(E+P)}+\left(1-\cos \theta_{\gamma}\right)\right]
$$

This becomes extremely peaked in the forward direction, $\cos \theta_{\gamma}=1$. For instance, while the term in square bracket is of order one for $\cos \theta_{\gamma}=-1$ it is of order $\sim 10^{-13}$ for linear collider energies of $500 \mathrm{GeV}$. In GRACE , integration is done with BASES [17] which is an adaptive Monte-Carlo program. For these particular cases one adapts the integration variables so that one fully picks up the singularities brought about by the hard photon collinear mass singularities. This step is therefore not as automatic as the previous ones in the calculation of the radiative corrections since one needs to judiciously choose the integration variables. For more details see 114 .

Stability of the result as concerns the cut-off $k_{c}$ is tested by varying the value of the cut-off $k_{c}$. We take $k_{c} \gg \lambda$ but, usually, much smaller than the maximum energy that the photon can have, for example a few percent of the centre-of-mass energy in $e^{+} e^{-}$processes. This is, typically, of the order of the photon energy that can be observed by a standard detector. In some multi-leg processes and for high energy it may be necessary to go down to values as low as $0.1 \mathrm{GeV}$ or even $10^{-3} \mathrm{GeV}$. One finds agreement within the precision of the Monte-Carlo which is at least better than 4 digits. From the view point of the computation a numerical cancellation occurs among the contribution of on the one hand, the virtual loop diagrams and soft photon emission, and on the other hand the hard photon emission. These individual contributions can be $1 \sim 10$ times larger than the tree cross section while the full $O(\alpha)$ correction, including virtual, bremmstrahlung and hard photon emission, is of order $1 \%$. The individual contributions should therefore be calculated extremely accurately. A loss of accuracy can also be caused if there is some severe cancellation among loop diagrams, as due to unitarity for example. In such cases one reverts to quadruple precision.

In $e^{+} e^{-}$processes where corrections from initial state radiation can be large it is possible to sum up the effect of multiple emission of photons, either through a structure function approach (see for instance 114]) or more sophisticated approaches that even takes into account the $p_{T}$ of the photon like that of the QEDPS approach[150]. 


\section{Checks on selected cross sections}

The previous sections have shown that the system passes highly non trivial checks for the calculation of the one-loop radiative corrections to $\mathcal{S M}$ processes. All those tests are internal tests within the system. To further establish the reliability of the system we have also performed comparisons with a number of one-loop electroweak calculations that have appeared in the literature. For all the comparisons we tune our input parameters to those given by the authors. Therefore one should remember that some of the results in the following tables are outdated due to the use of by now obsolete input parameters. All results refer to integrated cross sections with, in some cases, cuts on the scattering angle so as to avoid singularities in the forward direction. Apart from $e^{+} e^{-} \rightarrow t \bar{t}$ where a complete fully tuned comparison was conducted with high precision and includes the effect of hard photon radiation at $\mathcal{O}(\alpha)$, we compare the results of the virtual electroweak and soft photon bremsstrahlung $(V+S)$, taking the same cut-off, $k_{c}$, on the soft photon as specified in those references. We note in passing that since the GRACE system is adapted to multi-particle production, we can, contrary to some calculations, treat both the loop corrections and the bremsstrahlung correction within the same system. Let us also note that for all the processes we will consider below, we have taken the widths of all particles to zero since we never hit a pole.

\section{$9.1 \quad e^{+} e^{-} \rightarrow t \bar{t}$}

Table 5: Comparison of the total cross section $e^{+} e^{-} \rightarrow t \bar{t}$ between GRACE-loop and [151]. The corrections refer to the full one-loop electroweak corrections including hard photon radiation.

\begin{tabular}{|c|c|c|}
\hline$e^{+} e^{-} \rightarrow t \bar{t}$ & GRACE-loop & {$[151]$} \\
\hline$\sqrt{s}=500 \mathrm{GeV}$ & & \\
tree-level(in pb) & 0.5122751 & 0.5122744 \\
$\mathcal{O}(\alpha)$ (in pb) & 0.526371 & 0.526337 \\
$\delta$ (in \%) & 2.75163 & 2.74513 \\
& & \\
$\sqrt{s}=1 \mathrm{TeV}$ & & \\
tree-level(in pb) & 0.1559187 & 0.1559185 \\
$\mathcal{O}(\alpha)$ (in pb) & 0.171931 & 0.171916 \\
$\delta$ (in \%) & 10.2696 & 10.2602 \\
\hline
\end{tabular}

This process is an extension of the 2-fermion production program that has been successfully carried at LEP/SLC. The radiative corrections to this process first appeared in 152 
and then in 153]. A new computation has appeared very recently [151]. A dedicated tuned comparison between GRACE-loop and the program topfit 151] has recently been conducted at some depth including hard photon radiation and with the active participation of the authors of [151]. Details of the comparison are to be found in [154. Here we only show the excellent quality of the agreement for the total cross section including hard photons and we refer the reader to 154 for other comparisons concerning differential cross sections and forward-backward asymmetries. Let us point out however that the comparisons at the level of the differential cross sections agree within 8 digits before inclusion of the hard photon correction and to 7 digits when the latter are included. For the totally integrated cross section including hard photons this quality of agreement is somehow degraded but stays nonetheless excellent even at high energies. As Table 5 shows, the agreement is still better than 0.1permil.

The authors of [151] have also conducted a tuned comparison with another independent calculation based on[153]. Practically similar conclusions to the ones presented here are reached, see[155].

\section{$9.2 \quad e^{+} e^{-} \rightarrow W^{+} W^{-}$}

Table 6: Comparison of the total cross section $e^{+} e^{-} \rightarrow W^{+} W^{-}$between GRACE-1oop and [156]. The calculation includes full one-loop electroweak corrections, but no hard photon radiation.

\begin{tabular}{|c|c|c|}
\hline$e^{+} e^{-} \rightarrow W^{+} \bar{W}^{-}$ & GRACE-loop & {$[156]$} \\
\hline$\sqrt{s}=190 \mathrm{GeV}$ & & \\
tree-level(in pb) & 17.8623 & 17.863 \\
$\delta$ (in \%) & -9.4923 & -9.489 \\
& & \\
$\sqrt{s}=500 \mathrm{GeV}$ & & \\
tree-level(in pb) & 6.5989 & 6.599 \\
$\delta$ (in \%) & -12.743 & -12.74 \\
$\sqrt{s}=1 \mathrm{TeV}$ & & \\
tree-level(in pb) & 2.4649 & 2.465 \\
$\delta($ in \%) & -15.379 & -15.375 \\
\hline
\end{tabular}

This process is the most important electroweak process at LEP2 and constitutes one of the most important reactions for the linear collider. A few independent calculations [157] exist and the most recent ones agree better than the permil. To check the results given 
by GRACE, we have set our parameters to those appearing in Table 2 of the review [156]. The results refer to the total cross section but without the inclusion of the hard photon bremsstrahlung. As we see the agreement for energies ranging from LEP2 to $1 \mathrm{TeV}$ are about at least 0.1 permil.

\section{$9.3 \quad e^{+} e^{-} \rightarrow Z H$}

Table 7: Comparison of percentage correction to the total cross section $e^{+} e^{-} \rightarrow Z H$ between GRACE-loop and [93].

\begin{tabular}{|cc|c|c|}
\hline \multicolumn{2}{|c|}{$e^{+} e^{-} \rightarrow Z H$} & GRACE-loop & 93] \\
\hline$\sqrt{s}=500 \mathrm{GeV}$ & $M_{H}=100 \mathrm{GeV}$ & 4.15239 & 4.1524 \\
$\sqrt{s}=500 \mathrm{GeV}$ & $M_{H}=300 \mathrm{GeV}$ & 6.90166 & 6.9017 \\
$\sqrt{s}=1000 \mathrm{GeV}$ & $M_{H}=100 \mathrm{GeV}$ & -2.16561 & -2.1656 \\
$\sqrt{s}=1000 \mathrm{GeV}$ & $M_{H}=300 \mathrm{GeV}$ & -2.49949 & -2.4995 \\
$\sqrt{s}=1000 \mathrm{GeV}$ & $M_{H}=800 \mathrm{GeV}$ & 26.10942 & 26.1094 \\
$\sqrt{s}=2000 \mathrm{GeV}$ & $M_{H}=100 \mathrm{GeV}$ & -11.54131 & -11.5414 \\
$\sqrt{s}=2000 \mathrm{GeV}$ & $M_{H}=300 \mathrm{GeV}$ & -12.82256 & -12.8226 \\
$\sqrt{s}=2000 \mathrm{GeV}$ & $M_{H}=800 \mathrm{GeV}$ & 11.24680 & 11.2468 \\
\hline
\end{tabular}

This process is an important discovery channel for an intermediate mass Higgs at a moderate energy linear collider and could permit to study the properties of the Higgs. Three independent one-loop calculations exist [93, 94, 95] which all agree beyond the precision of any future linear collider. A comparison was conducted against the calculation in 93. where one of the authors has provided us with more precise numbers than those appearing in Table 1 of 93$]^{12}$. Table 7 shows that the results given by our system

\footnotetext{
${ }^{12}$ We thank A. Denner for providing us with the correct $M_{W}$ masses used in this table. Beside the input given in [93], $M_{W}$ is crucial for a precise comparison. The following $M_{W}$ masses have been used: $M_{W}=$ $80.231815 \mathrm{GeV}\left(M_{H}=100 \mathrm{GeV}\right), M_{W}=80.159313 \mathrm{GeV}\left(M_{H}=300 \mathrm{GeV}\right), M_{W}=80.081409 \mathrm{GeV}\left(M_{H}=\right.$ $800 \mathrm{GeV})$.
} 
GRACE-1oop and those of [93] agree on all digits. This means that the radiatively corrected cross sections at different energies for a Higgs mass ranging from the light to the heavy agree within at least 6 digits. The corrections refer to the full one-loop electroweak corrections but without hard photon radiation.

\section{$9.4 \gamma \gamma \rightarrow t \bar{t}$}

Table 8: Comparison of the total cross section $\gamma \gamma \rightarrow t \bar{t}$ between GRACE-1oop and [158] include full one-loop electroweak corrections at one-loop, but no hard (final) photon radiation.

\begin{tabular}{|c|c|c|}
\hline$\gamma \gamma \rightarrow t \bar{t}$ & GRACE-loop & {$[158]$} \\
\hline$\sqrt{s}=350 \mathrm{GeV}$ & & \\
tree-level (in pb) & 0.332477 & 0.33248 \\
$\delta$ (in \%) & -6.889 & -6.88 \\
& & \\
$\sqrt{s}=500 \mathrm{GeV}$ & & \\
tree-level(in pb) & 0.904371 & 0.90439 \\
$\delta$ (in \%) & -4.824 & -4.82 \\
$\sqrt{s}=1 \mathrm{TeV}$ & & \\
tree-level (in pb) & 0.434459 & 0.43447 \\
$\delta$ (in \%) & -5.633 & -5.63 \\
& & \\
\hline
\end{tabular}

The comparison has been made with Table 1 of [158 without any convolution over any photon spectra. $M_{H}=150 \mathrm{GeV}$ so as to avoid the Higgs resonance. As we see the agreement is very good, it is just limited by the precision of the numbers provided in [158.

\section{$9.5 \gamma \gamma \rightarrow W^{+} W^{-}$}

The first complete calculation of the electroweak radiative corrections to $\gamma \gamma \rightarrow W^{+} W^{-}$ has been performed in [159]. Jikia has performed a full $\mathcal{O}(\alpha)$ calculation, including hard photon radiation [160. Comparison has been made on the one hand with Table 1 of [159] without convolution over any photon spectra as well as with Table 2 of [160. In both comparisons we considered the total integrated cross section but with no inclusion of the hard photon radiation which in any case is not treated in [159. Because the 
Table 9: Comparison for $\gamma \gamma \rightarrow W^{+} W^{-}$between GRACE-loop and [159] and GRACE-loop and [160]. No hard (final) photon radiation is included. When not stated the cross sections and corrections refer to the total cross section with no angular cut.

\begin{tabular}{|c|c|c|}
\hline$\gamma \gamma \rightarrow W^{+} W^{-}, M_{W}=80.36 \mathrm{GeV} M_{H}=300 \mathrm{GeV}$ & GRACE-loop & 160 \\
\hline $\begin{array}{c}\sqrt{s}=500 \mathrm{GeV} \\
\text { tree-level(in pb) } \\
\delta(\text { in } \%)\end{array}$ & $\begin{array}{l}77.497 \\
-10.06\end{array}$ & $\begin{array}{l}77.50 \\
-10.1\end{array}$ \\
\hline $\begin{array}{c}\sqrt{s}=1 \mathrm{TeV} \\
\text { tree-level(in pb) } \\
\delta(\text { in } \%)\end{array}$ & $\begin{array}{l}79.995 \\
-18.73\end{array}$ & $\begin{array}{l}79.99 \\
-18.7\end{array}$ \\
\hline $\begin{array}{c}\sqrt{s}=2 \mathrm{TeV} \\
\text { tree-level(in pb) } \\
\delta(\text { in } \%)\end{array}$ & $\begin{array}{l}80.531 \\
-27.25\end{array}$ & $\begin{array}{l}80.53 \\
-27.2\end{array}$ \\
\hline $\begin{array}{c}\sqrt{s}=2 \mathrm{TeV} 60^{\circ}<\theta<120^{\circ} \\
\text { tree-level(in pb) } \\
\delta(\text { in } \%)\end{array}$ & $\begin{array}{c}0.39356 \\
-75.6827\end{array}$ & $\begin{array}{l}0.3936 \\
-75.6\end{array}$ \\
\hline$M_{W}=80.333 \mathrm{GeV} M_{H}=250 \mathrm{GeV}$ & GRACE-loop & [159 \\
\hline $\begin{array}{c}\sqrt{s}=500 \mathrm{GeV} \\
\text { tree-level(in pb) } \\
\delta(\text { in \%) }\end{array}$ & $\begin{array}{l}77.552 \\
-3.376\end{array}$ & $\begin{array}{r}77.55 \\
-3.38\end{array}$ \\
\hline $\begin{array}{c}\sqrt{s}=1 \mathrm{TeV} \\
\text { tree-level(in pb) } \\
\delta(\text { in } \%)\end{array}$ & $\begin{array}{l}80.049 \\
-7.087\end{array}$ & $\begin{array}{r}80.05 \\
-7.08\end{array}$ \\
\hline
\end{tabular}


fermionic contribution is extremely small compared to the bosonic contribution in the radiative correction to the total cross section, we also looked at the correction with an angular cut on the outgoing $W$ as check on the fermionic correction. As can be seen from Table 9 the agreement is just limited by the precision of the numbers provided in [159] and [160]. Note that in [160], the correction is split between the bosonic corrections and the fermionic corrections. When considering the total cross section the latter are much too small and are below the precision with which the bosonic corrections are displayed in [160. Therefore given the precision of the data in [160] the corrections are essentially given by the bosonic part for the total cross section. For the entry with the angular cut, the fermionic corrections are not negligible.

\section{6 $\quad e \gamma \rightarrow W \nu_{e}$}

Table 10: Comparison of the total cross section $e \gamma \rightarrow W \nu_{e}$ between GRACE-1oop and [161].

\begin{tabular}{|c|c|c|}
\hline$e \gamma \rightarrow W \nu_{e}$ & GRACE-loop & {$[161]$} \\
\hline$\sqrt{s}=500 \mathrm{GeV}$ & & \\
tree-level(in pb) & 36.5873 & 36.587 \\
$\delta($ in \%) & -12.2803 & -12.281 \\
& & \\
$\sqrt{s}=2 \mathrm{TeV}$ & & \\
tree-level(in pb) & 43.9368 & 43.937 \\
$\delta($ in \%) & -19.0917 & -19.092 \\
\hline
\end{tabular}

The comparison shown in Table. [10] is made on the total cross section $\left(0^{\circ} \leq \theta \leq 180^{\circ}\right)$ based on Table 5.1 of [161. No convolution on the photon spectra is applied nor is the hard photon bremsstrahlung included. The agreement is rather excellent.

\section{7 $e \gamma \rightarrow e Z$}

The comparison shown in Table. 11] is made with Table 5.3 of [162]. No convolution on the photon spectra is applied nor is the hard photon bremsstrahlung included. The agreement is excellent. 
Table 11: Comparison of the total cross section e $\rightarrow e Z$ between GRACE-1oop and [162].

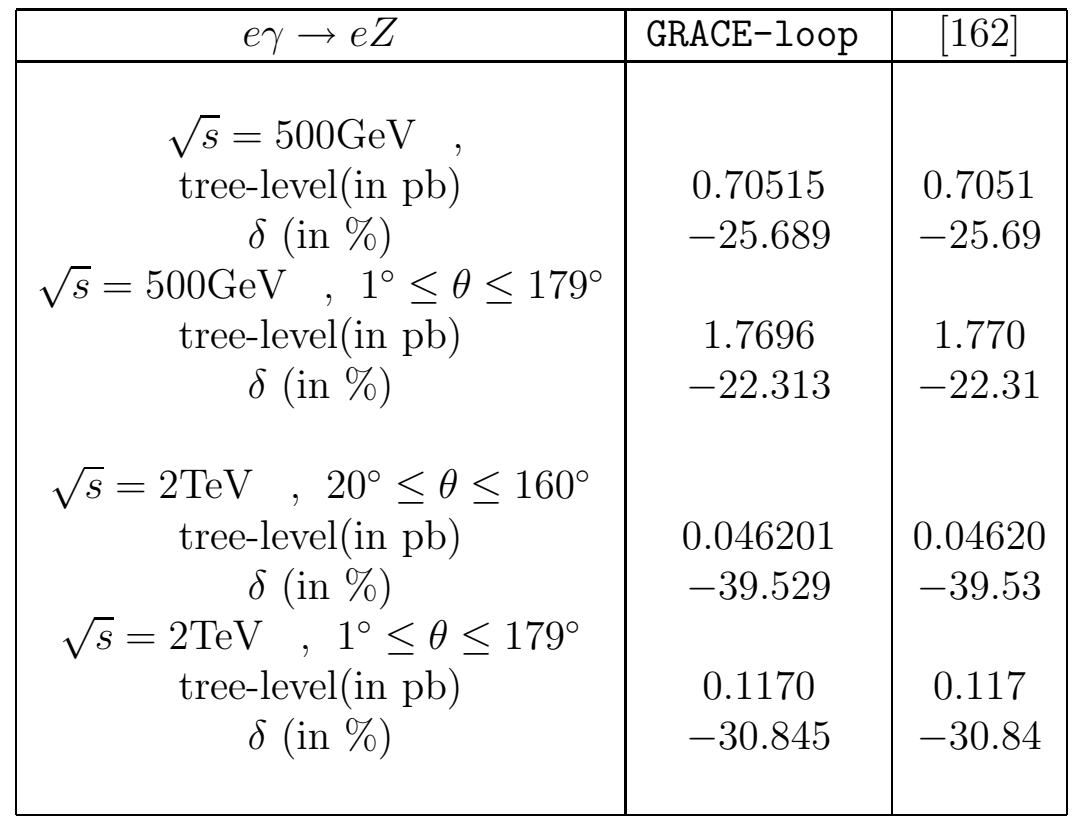

\section{$9.8 \quad W^{+} W^{-} \rightarrow W^{-} W^{+}$}

This is one of the most difficult $2 \rightarrow 2$ processes in the $\mathcal{S M}$ ever to be calculated. As discussed previously the number of diagrams at one-loop is of the order 1000. Moreover very subtle gauge cancellations take place especially as the energy of the participating $W$ 's increases. The most complete calculation has been performed in [163] and the code is freely available at www.hep-processes.de. However, hard photon radiation is not included. Following [163] we have compared our results with those of the code by requiring a cut on the forward-backward direction such that the integration over the scattering angle is over $10^{\circ} \leq \theta \leq 170^{\circ}$. Moreover we have considered two cuts on the photon energy (for the bremsstrahlung part), $k_{c}=.05 \sqrt{s}$ and $k_{c}=.5 \sqrt{s}^{13}$. The Higgs in this comparison is light, see 163. for a justification on this issue. Having at our disposal the code, a tuned comparison could be performed. We can see from Table 12 that at centre-of-mass energy of the $W$ pair of $2 \mathrm{TeV}$ one reaches agreement over 6 digits, 4-5 digits for $\sqrt{s}=5 \mathrm{TeV}$ but "only" 3 digits agreement for $\sqrt{s}=10 \mathrm{TeV}$. Note that even in this case this means that the radiative corrections are known to about 0.1 permil. This very high energy for the $W W$ would probably never be reached. Moreover as the authors of 163 warn, for this kind of energy an integration in quadruple precision is probably already mandatory.

\footnotetext{
${ }^{13}$ These cut-off photon energies are much higher than those recommended in section 8.3 However we stick to these values to comply with those chosen in Ref. [163.
} 
Table 12: Comparison of the total (unpolarised) cross section $W^{+} W^{-} \rightarrow W^{-} W^{+}$between GRACE-loop and [163]. $M_{H}=100 \mathrm{GeV}$. For the cuts see the text.

\begin{tabular}{|c|c|c|}
\hline$W^{+} W^{-} \rightarrow W^{-} W^{+}$ & GRACE-1oop & 163 \\
\hline$k_{c}=.05 \sqrt{s}$ & & \\
\hline$\sqrt{s}=2 \mathrm{TeV}$ & & \\
\hline tree-level(in pb) & 77.17067 & 77.17067 \\
\hline$\delta($ in $\%)$ & -21.0135 & -21.0135 \\
\hline$\sqrt{s}=5 \mathrm{TeV}$ & & \\
\hline tree-level(in pb) & 14.2443 & 14.2443 \\
\hline$\delta($ in $\%)$ & -57.1567 & -57.1556 \\
\hline$\sqrt{s}=10 \mathrm{TeV}$ & & \\
\hline tree-level(in pb) & 3.644573 & 3.644573 \\
\hline$\delta($ in $\%)$ & -93.9942 & -94.0272 \\
\hline$k_{c}=.5 \sqrt{s}$ & & \\
\hline$\sqrt{s}=2 \mathrm{TeV}$ & & \\
\hline tree-level(in pb) & 77.17067 & 77.17067 \\
\hline$\delta($ in $\%)$ & -17.23988 & -17.23989 \\
\hline$\sqrt{s}=5 \mathrm{TeV}$ & & \\
\hline tree-level(in pb) & 14.24434 & 14.24434 \\
\hline$\delta($ in $\%)$ & -49.9736 & -49.9724 \\
\hline$\sqrt{s}=10 \mathrm{TeV}$ & & \\
\hline tree-level(in pb) & 3.644574 & 3.644573 \\
\hline$\delta($ in $\%)$ & -83.9247 & -83.9577 \\
\hline
\end{tabular}


Therefore it is fair to conclude that one has for this reaction an excellent agreement. Note that GRACE-loop automatic calculation is the first confirmation of the result of [163]. 


\section{Conclusions}

Precision measurements in high-energy physics need to be matched by very accurate theoretical predictions. This requires that one performs calculations that go beyond the tree-level approximation. Moreover with the increase in energy, as will be available at the upcoming colliders, some important multi-particle final states beyond the usual $2 \rightarrow 2$ processes become important physical observables. Higgs production at the linear collider is such an example. Calculation of multi-loop and multi-leg processes involves the computations of thousands of diagrams, with the property that the topologies that appear as the order of perturbation theory increases become extremely arduous. An example is the treatment of the $N$-point functions beyond the box or two-loop diagrams. A calculation by hand is obviously no longer possible. Although a few of the steps involved in these computations are now done with the help of computers, it has become almost a necessity to perform the whole chain in the calculation of these complex cross sections by a computer with a minimum of human intervention so as to avoid any risk of error. We have in this review taken GRACE-1oop as a prototype of such a fully automated system and described in detail its workings and performance in evaluating one-loop processes in the electroweak theory. The general strategy of constructing a code like this can of course be applied to other systems and we have discussed some of them. We have also reviewed in some detail the most important modules and components that an automated system must have. Since there are a few fully automated systems at tree-level, the emphasis in this report has been on the extension to and the implementation of the one-loop corrections. Central to this implementation is the library for the reduction of the tensorial loop integrals and also the reduction of the higher $N$-point functions $(N=5,6)$ to lower $N$-point scalar integrals. We have presented an algorithm which is now fully functional in GRACE-loop .

Another crucial aspect of a fully automated calculation is the possibility of checking the results of its output. In an automated tree-level system this is almost trivial and is usually provided by switching between the Feynman gauge and the unitary gauge. As we argued the unitary gauge or the usual general $R_{\xi}$ linear gauge are not suitable at all for an implementation in a multi-purpose one-loop automatic code. Other important checks are the ultraviolet and infrared finiteness tests, but the gauge-parameter independence check is most powerful. This is the reason a part of this review has been devoted to renormalisation in the $\mathcal{S M}$ within a non-linear gauge. The non-linear gauge that we exploit introduces 5 gauge parameters. This gauge fixing modifies a large number of vertices in the bosonic sector but can be chosen so as to leave the propagators of all gauge bosons as simple as in the standard linear 't Hooft-Feynman gauge. Technically this means that the structure of any one-loop $N$-point function is not more involved than what it is with fermionic loops and therefore that many libraries for these functions need not be extended. To show that one-loop automated systems have now become completely functional and trustworthy as concerns the treatment of any $2 \rightarrow 2$ processes we have presented conclusive tests on the 
finiteness, both infrared and ultraviolet, and the gauge-parameter independence of the results pertaining to some 26 processes. These checks are verified with a precision that attains at least 20 digits. We have also used the new system to carry further comparison on radiative corrections to a few processes that have appeared in the literature. This selection includes heavy fermion production, vector boson and Higgs boson production in both $e^{+} e^{-}, \gamma \gamma$ and $e \gamma$ machines as well as the very challenging $W W$ scattering process. For the latter we provide the first check to the complete calculation that has appeared in the literature. In all cases we find excellent agreement.

This shows that the automatic system of calculating radiative corrections numerically has now all the ingredients to tackle $2 \rightarrow 3$ processes confidently. In the last two years, major progress has been made in this area thanks to the automated system GRACE-1oop and the package FeynArts-FeynCalc-FormCalc. Most important processes for Higgs production at the linear collider, $e^{+} e^{-} \rightarrow \nu_{e} \bar{\nu}_{e} H$ [1, 2, 3], $e^{+} e^{-} \rightarrow e^{+} e^{-} H$ [42, $e^{+} e^{-} \rightarrow$ $Z H H$ [43, 44], $e^{+} e^{-} \rightarrow t \bar{t} H$ [45, 46, 47], $\gamma \gamma \rightarrow t \bar{t} H$ [48] as well as $e^{+} e^{-} \rightarrow e^{+} e^{-} \gamma$ [4] have been computed and for most of them checked against each other, thanks to the automatic systems. GRACE-loop is now in a position to compute one-loop $2 \rightarrow 4$ processes. We have in fact already presented some results pertaining to $e^{+} e^{-} \rightarrow \nu_{e} \bar{\nu}_{e} H H$ [50] and some preliminary ones for the four-fermion final state $e^{+} e^{-} \rightarrow \mu^{-} \bar{\nu}_{\mu} u \bar{d}$ at one-loop [52]. Based on the FeynArts-FeynCalc-FormCalc package the one-loop correction for a certain class of $e^{+} e^{-} \rightarrow 4 f$ has also been achieved [51] recently.

To improve the efficiency of the system for applications to one-loop corrections for processes with more than 2 particles in the final state, one should seek a derivation based on helicity amplitudes. At tree-level this has been nicely implemented in GRACE and applied to processes up to 6 particles in the final state. Moreover a derivation based on helicity amplitudes allows the implementation of full spin-correlation, for processes when the final particle is unstable. At the one-loop level, another advantage is that it would allow the calculation by the system of processes that are not generated at tree-level, such as $\gamma \gamma \rightarrow \gamma \gamma, \gamma \gamma \rightarrow Z Z, Z \rightarrow 3 \gamma, H \rightarrow \gamma \gamma$. The working version of GRACE-loop can not handle such processes since the one-loop correction are calculated as products of tree-level and one-loop matrix elements. A version of GRACE-loop which is being developed is based on helicity amplitudes. Preliminary results are encouraging. This new version will also be used as an additional test on the results given by the traditional method of squaring matrix elements.

\section{Acknowledgment}

This work is part of a collaboration between the GRACE project in the Minami-Tateya group and LAPTH. D. Perret-Gallix and Y. Kurihara deserve special thanks for their

contribution. We also thank M. Kuroda and J.A.M. Vermaseren for a critical reading of the manuscript. This work was supported in part by the Japan Society for Promotion of Science (JSPS) under the Grant-in-Aid for scientific Research B(N. 14340081), PICS 397 
and The GDRi-ACPP of the French National Centre for Scientific Research (CNRS). 


\section{Appendices}

\section{A Specific form of the BRST transformations}

The action of the BRST transformations is derived as a generalisation of the usual gauge transformations. The ghost fields corresponding to the four gauge bosons write in terms of the ghosts of the $S U(2) \times U(1)$ fields as:

$$
\begin{aligned}
c^{ \pm} & =\frac{1}{\sqrt{2}}\left(c^{1} \mp i c^{2}\right) \\
c^{A} & =s_{W} c^{3}+c_{W} c^{B} \\
c^{Z} & =c_{W} c^{3}-s_{W} c^{B} .
\end{aligned}
$$

One obtains

$$
\begin{aligned}
\delta_{\mathrm{BRS}} W_{\mu}^{ \pm} & =\partial_{\mu} c^{ \pm} \mp i e\left[\left(\left(A_{\mu}+\frac{c_{W}}{s_{W}} Z_{\mu}\right) c^{ \pm}-\left(c^{A}+\frac{c_{W}}{s_{W}} c^{Z}\right) W_{\mu}^{ \pm}\right]\right. \\
\delta_{\mathrm{BRS}} Z_{\mu} & =\partial_{\mu} c^{Z}-i g c_{W}\left(W_{\mu}^{+} c^{-}-W_{\mu}^{-} c^{+}\right) \\
\delta_{\mathrm{BRS}} A_{\mu} & =\partial_{\mu} c^{A}-i e\left(W_{\mu}^{+} c^{-}-W_{\mu}^{-} c^{+}\right) .
\end{aligned}
$$

Likewise by considering the gauge transformation on the Higgs doublet one gets

$$
\begin{aligned}
\delta_{\mathrm{BRS}} H & =-\frac{g}{2}\left(c^{-} \chi^{+}+c^{+} \chi^{-}\right)-\frac{e}{2 s_{W} c_{W}} c^{Z} \chi_{3}, \\
\delta_{\mathrm{BRS}} \chi_{3} & =-\frac{i g}{2}\left(-c^{+} \chi^{-}+c^{-} \chi^{+}\right)+\frac{e}{2 s_{W} c_{W}} c^{Z}(v+H), \\
\delta_{\mathrm{BRS}} \chi^{ \pm} & =\frac{g}{2}\left(v+H \mp i \chi_{3}\right) c^{ \pm} \pm i e \chi^{ \pm}\left(c^{A}+\frac{c_{W}^{2}-s_{W}^{2}}{2 s_{W} c_{W}} c^{Z}\right) .
\end{aligned}
$$

To find the transformation for the ghost fields, notice that the BRST transformation is nilpotent. For instance from $\left(\delta_{\mathrm{BRS}}\right)^{2} W_{\mu}^{i}=0$ one gets $\delta_{\mathrm{BRS}} c^{i}$. Indeed more generally one has, for any group,

$$
\delta_{\mathrm{BRS}} A_{\mu}^{i}=D_{\mu} c^{i}=\partial_{\mu} c^{i}+g\left[A_{\mu}, c\right]^{i} \rightarrow \delta_{\mathrm{BRS}} c^{i}=-g \frac{1}{2}[c, c]^{i}
$$

Care should be taken that $\delta_{\mathrm{BRS}}$ being a fermion operator the graded Leibnitz rule applies: $\delta_{\mathrm{BRS}}(X Y)=\left(\delta_{\mathrm{BRS}} X\right) Y \pm X\left(\delta_{\mathrm{BRS}} Y\right)$ where the minus sign applies if $\mathrm{X}$ has an odd number of ghosts or antighosts, note also that $\left(c^{i}\right)^{2}=0$.

In our case this implies

$$
\delta_{\mathrm{BRS}} c^{B}=0 \quad \delta_{\mathrm{BRS}} c^{i}=-\frac{1}{2} g \epsilon_{i j k} c^{j} c^{k}
$$


and thus

$$
\begin{aligned}
& \delta_{\mathrm{BRS}} c^{ \pm}=\mp i g c^{ \pm}\left(s_{W} c^{A}+c_{W} c^{Z}\right) \\
& \delta_{\mathrm{BRS}} c^{A}=+i e c^{+} c^{-} \\
& \delta_{\mathrm{BRS}} c^{Z}=+i g c_{W} c^{+} c^{-}
\end{aligned}
$$

The transformation for the anti-ghost field is defined through the auxiliary $B$ field of the gauge functions,

$$
\delta_{\mathrm{BRS}} \bar{c}^{i}=B^{i}
$$

\section{B Feynman Rules}

The basic Feynman rules follow the so-called Kyoto convention[90]. A particle at the endpoint enters the vertex. For instance, if a line is denoted as $W^{+}$, then the line shows either the incoming $W^{+}$or the outgoing $W^{-}$. The momentum assigned to a particle is defined as inward except for the case of a ghost particle for which the momentum is defined along the flow of its ghost number, as will be shown in the figures. 


\section{B.1 Propagators}

\begin{tabular}{ll}
\hline$W^{ \pm}$ & $\frac{1}{k^{2}-M_{W}^{2}}\left(g_{\mu \nu}-\left(1-\xi_{W}\right) \frac{k_{\mu} k_{\nu}}{k^{2}-\xi_{W} M_{W}^{2}}\right)$ \\
$Z$ & $\frac{1}{k^{2}-M_{Z}^{2}}\left(g_{\mu \nu}-\left(1-\xi_{Z}\right) \frac{k_{\mu} k_{\nu}}{k^{2}-\xi_{Z} M_{Z}^{2}}\right)$ \\
$A$ & $\frac{1}{k^{2}}\left(g_{\mu \nu}-\left(1-\xi_{A}\right) \frac{k_{\mu} k_{\nu}}{k^{2}}\right)$ \\
\hline$f$ & $\frac{-1}{\not k-m_{f}}$ \\
\hline$H$ & $\frac{-1}{k^{2}-M_{H}^{2}}$ \\
\hline$\chi^{ \pm}$ & $\frac{-1}{k^{2}-\xi_{W} M_{W}^{2}}$ \\
\hline$\chi_{3}$ & $\frac{-1}{k^{2}-\xi_{Z} M_{Z}^{2}}$ \\
\hline$c^{ \pm}$ & $\frac{-1}{k^{2}-\xi_{W} M_{W}^{2}}$ \\
\hline$c^{Z}$ & $\frac{-1}{k^{2}-\xi_{Z} M_{Z}^{2}}$ \\
\hline$c^{A}$ & $\frac{-1}{k^{2}}$ \\
\hline
\end{tabular}

\section{B.2 Vector-Vector-Vector}

\begin{tabular}{lll}
\hline$p_{1}(\mu)$ & $p_{2}(\nu)$ & $p_{3}(\rho)$ \\
\hline
\end{tabular}

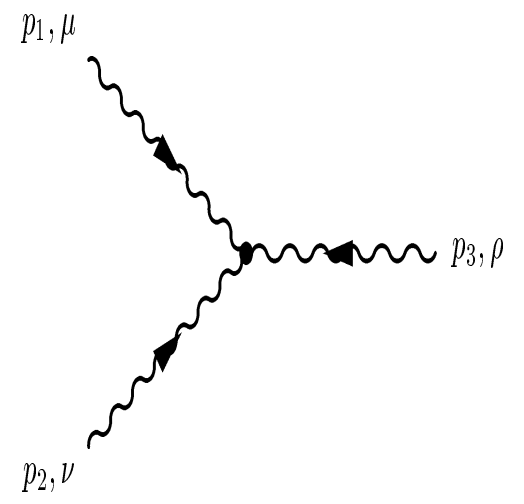

$$
\begin{aligned}
W^{-} \quad W^{+} \quad A \quad & e\left[g^{\mu \nu}\left(p_{1}-p_{2}\right)^{\rho}\right. \\
& +\left(1+\tilde{\alpha} / \xi_{W}\right)\left(p_{g^{\nu}}^{\mu \rho}-p_{3}^{\mu} g^{\nu \rho}\right) \\
& \left.+\left(1-\tilde{\alpha} / \xi_{W}\right)\left(p_{2}^{\mu} g^{\nu \rho}-p_{1}^{\nu} g^{\mu \rho}\right)\right]
\end{aligned}
$$

\begin{aligned} \hline$W^{-} \quad W^{+} \quad Z \quad & e \frac{c_{W}}{s_{W}}\left[g^{\mu \nu}\left(p_{1}-p_{2}\right)^{\rho}\right. \\ & +\left(1+\tilde{\beta} / \xi_{W}\right)\left(p_{3}^{\nu} g^{\mu \rho}-p_{3}^{\mu} g^{\nu \rho}\right) \\ & \left.+\left(1-\tilde{\beta} / \xi_{W}\right)\left(p_{2}^{\mu} g^{\nu \rho}-p_{1}^{\nu} g^{\mu \rho}\right)\right]\end{aligned}$




\section{B.3 Vector-Vector-Scalar}

\begin{tabular}{|c|c|c|c|c|}
\hline \multirow{3}{*}{$p_{1}, \mu$} & $p_{1}(\mu)$ & $p_{2}(\nu)$ & $p_{3}$ & \\
\hline & $W^{ \pm}$ & $A$ & $\chi^{\mp}$ & $\mp i e M_{W}(1-\tilde{\alpha}) g^{\mu \nu}$ \\
\hline & $W^{ \pm}$ & $Z$ & $\chi^{\mp}$ & $\pm i e \frac{1}{s_{W} c_{W}} M_{W}\left(1-c_{W}^{2}(1-\tilde{\beta})\right) g^{\mu \nu}$ \\
\hline \multirow[b]{2}{*}{$p_{2}, \nu$} & $W^{-}$ & $W^{+}$ & $H$ & $e \frac{1}{s_{W}} M_{W} g^{\mu \nu}$ \\
\hline & $Z$ & $Z$ & $H$ & $e \frac{1}{s_{W} c_{W}^{2}} M_{W} g^{\mu \nu}$ \\
\hline
\end{tabular}

\section{B.4 Scalar-Scalar-Vector}

\begin{tabular}{lccc}
\hline$p_{1}$ & $p_{2}$ & $p_{3}(\mu)$ & \\
\hline$H$ & $\chi^{\mp}$ & $W^{ \pm}$ & $i e \frac{1}{2 s_{W}}\left[(1-\tilde{\delta}) p_{2}^{\mu}-(1+\tilde{\delta}) p_{1}^{\mu}\right]$ \\
\hline$\chi_{3}$ & $\chi^{\mp}$ & $W^{ \pm}$ & $\pm e \frac{1}{2 s_{W}}\left[(1-\tilde{\kappa}) p_{2}^{\mu}-(1+\tilde{\kappa}) p_{1}^{\mu}\right]$ \\
\hline$\chi^{-}$ & $\chi^{+}$ & $A$ & $e\left(p_{2}-p_{1}\right)^{\mu}$ \\
\hline & & & $e \frac{c_{W}^{2}-s_{W}^{2}}{2 s_{W} c_{W}}\left(p_{2}-p_{1}\right)^{\mu}$ \\
$\chi^{-}$ & $\chi^{+}$ & $Z$ & $i e \frac{1}{2 s_{W} c_{W}}\left[(1-\tilde{\varepsilon}) p_{2}^{\mu}-(1+\tilde{\varepsilon}) p_{1}^{\mu}\right]$ \\
\hline$H$ & $\chi_{3}$ & $Z$ &
\end{tabular}

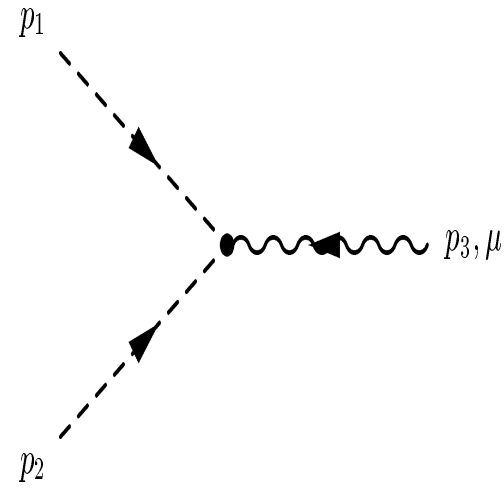




\section{B.5 Scalar-Scalar-Scalar}

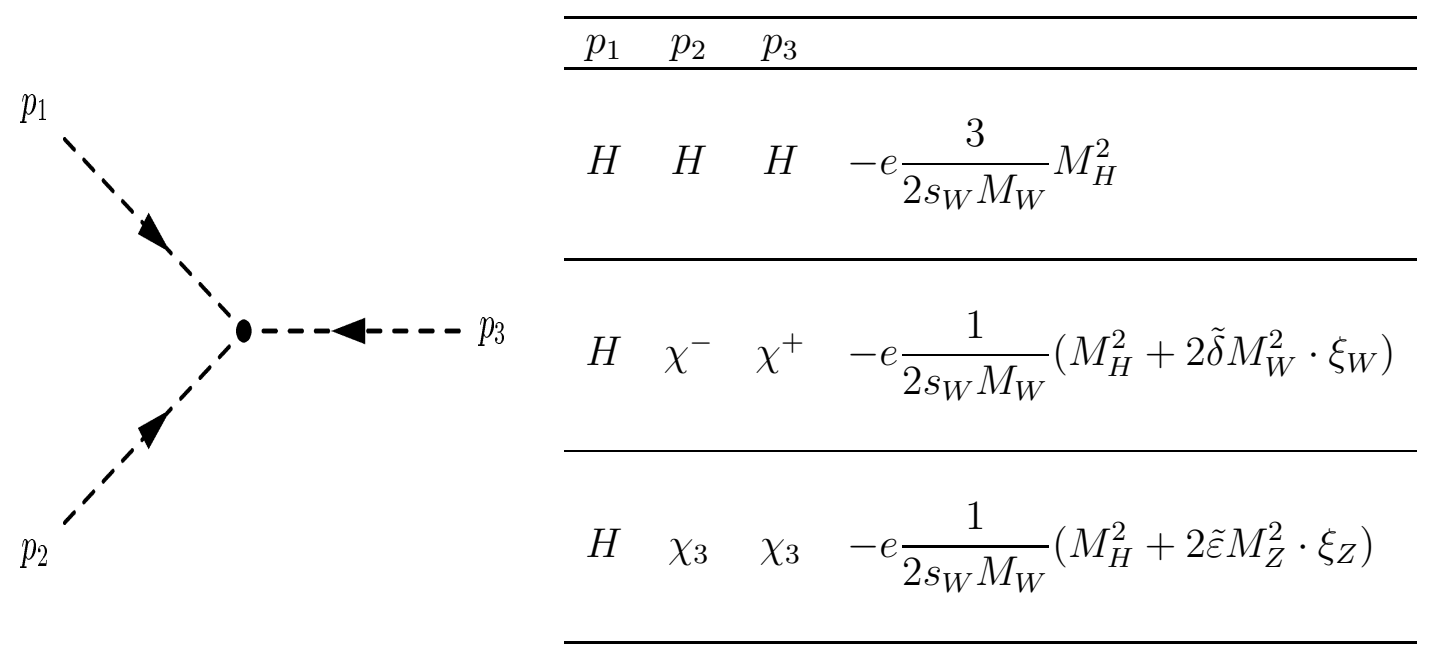

\section{B.6 Vector-Vector-Vector-Vector}

$$
\begin{array}{cccc}
\hline p_{1}(\mu) & p_{2}(\nu) & p_{3}(\rho) & p_{4}(\sigma) \\
\hline W^{+} & W^{-} & A & A \\
e^{2}\left[-2 g^{\mu \nu} g^{\rho \sigma}+\left(1-\tilde{\alpha}^{2} / \xi_{W}\right)\left(g^{\mu \rho} g^{\nu \sigma}+g^{\mu \sigma} g^{\nu \rho}\right)\right] \\
\hline W^{+} & W^{-} & A & Z
\end{array}
$$

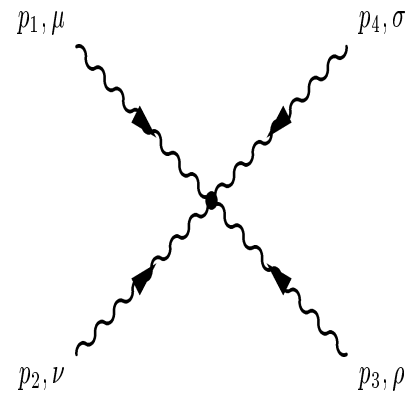

$$
e^{2} \frac{c_{W}}{s_{W}}\left[-2 g^{\mu \nu} g^{\rho \sigma}+\left(1-\tilde{\alpha} \tilde{\beta} / \xi_{W}\right)\left(g^{\mu \rho} g^{\nu \sigma}+g^{\mu \sigma} g^{\nu \rho}\right)\right]
$$

$$
\begin{array}{llll}
W^{+} & W^{-} & Z & Z
\end{array}
$$$$
e^{2} \frac{c_{W}^{2}}{s_{W}^{2}}\left[-2 g^{\mu \nu} g^{\rho \sigma}+\left(1-\tilde{\beta}^{2} / \xi_{W}\right)\left(g^{\mu \rho} g^{\nu \sigma}+g^{\mu \sigma} g^{\nu \rho}\right)\right]
$$

$$
\begin{aligned}
& W^{+} W^{-} W^{-} W^{+} \\
& \quad-e^{2} \frac{1}{s_{W}^{2}}\left[-2 g^{\mu \sigma} g^{\nu \rho}+\left(g^{\mu \rho} g^{\nu \sigma}+g^{\mu \nu} g^{\rho \sigma}\right)\right]
\end{aligned}
$$


B.7 Vector-Vector-Scalar-Scalar

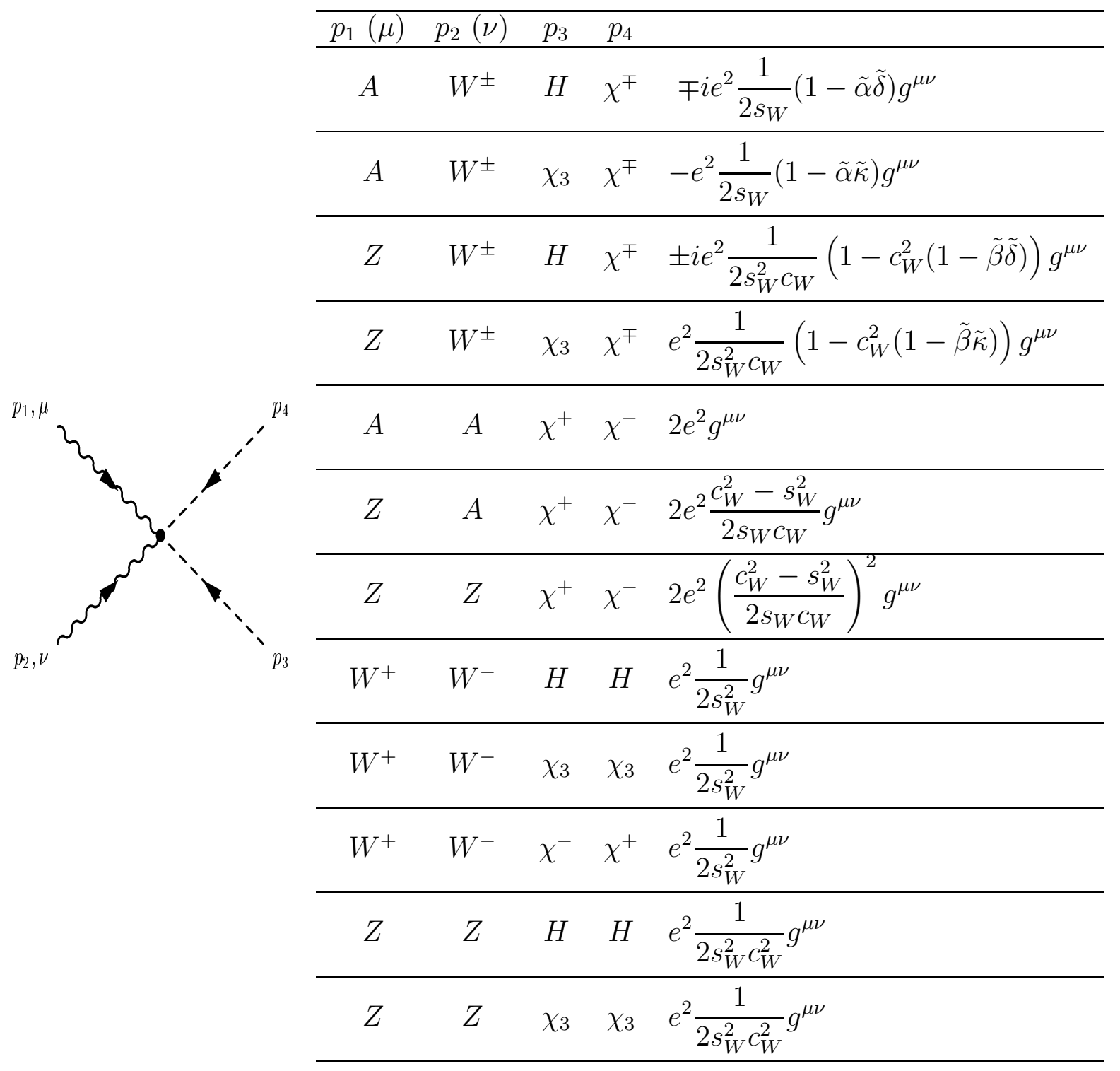




\section{B.8 Scalar-Scalar-Scalar-Scalar}

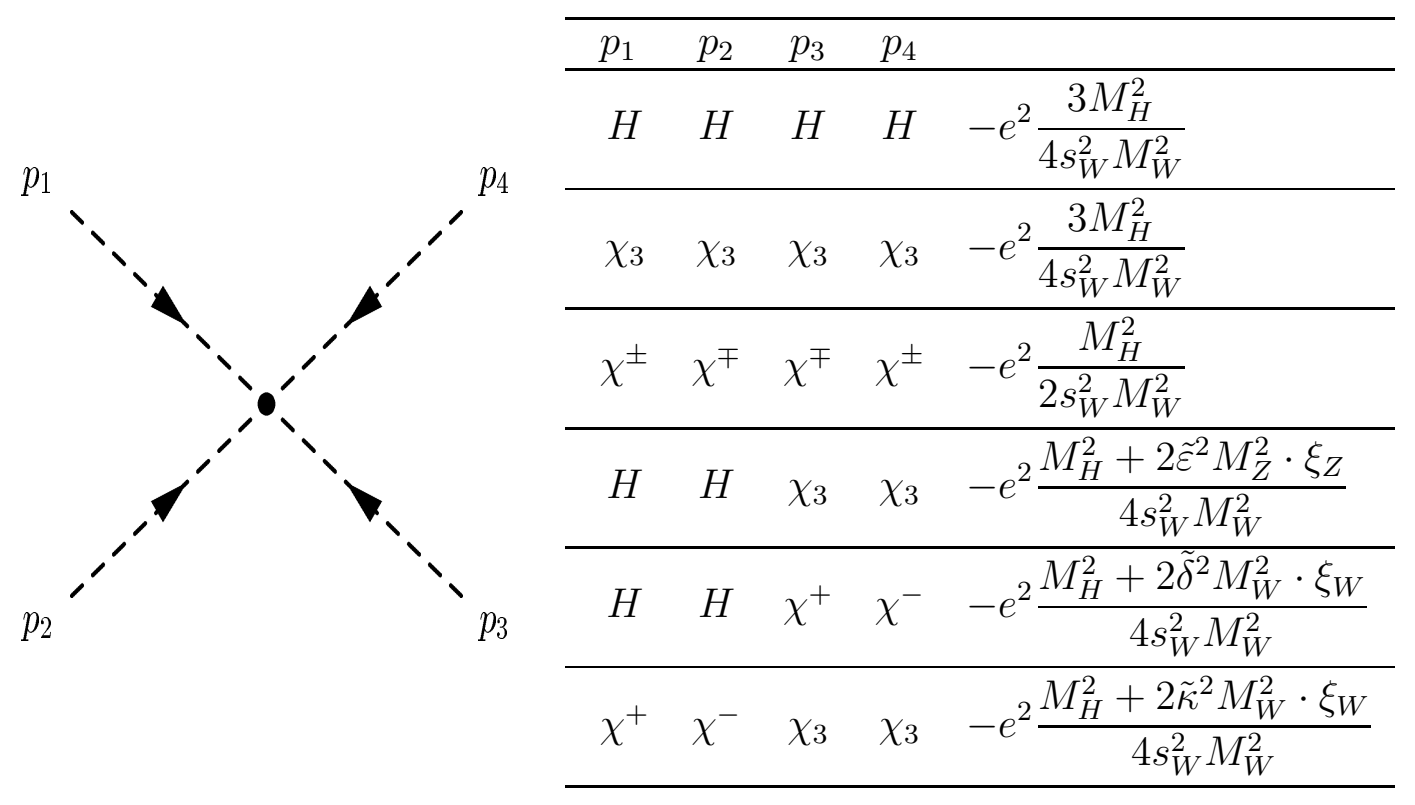

\section{B.9 Fermion-Fermion-Vector}

Fermion mixing is not shown here. Colour for quarks is also not explicit and should be taken into account when appropriate.

\begin{tabular}{|c|c|cc|c|cc|}
\hline$f$ & & $I_{3}$ & $Q_{f}$ & & $I_{3}$ & $Q_{f}$ \\
\hline$U$ & $u, c, t$ & $\frac{1}{2}$ & $\frac{2}{3}$ & $\nu_{e}, \nu_{\mu}, \nu_{\tau}$ & $\frac{1}{2}$ & 0 \\
\hline$D$ & $d, s, b$ & $-\frac{1}{2}$ & $-\frac{1}{3}$ & $e, \mu, \tau$ & $-\frac{1}{2}$ & -1 \\
\hline
\end{tabular}

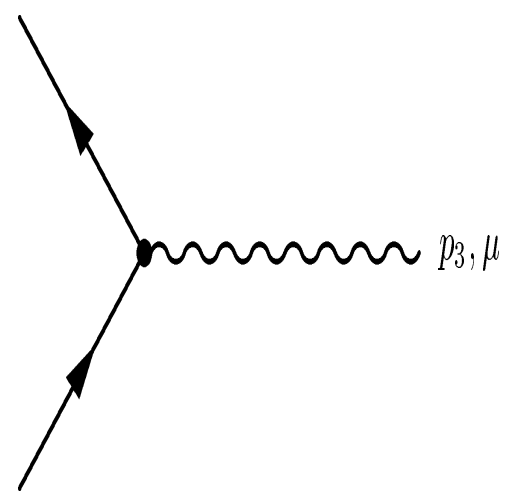

\begin{tabular}{cccc}
\hline$p_{1}$ & $p_{2}$ & $p_{3}(\mu)$ \\
\hline $\bar{f}$ & $f$ & $A$ & $e Q_{f} \gamma^{\mu}$ \\
\hline $\bar{f}$ & $f$ & $Z$ \\
& \multicolumn{2}{c}{$e \frac{1}{2 s_{W} c_{W}} \gamma^{\mu}\left(I_{3}\left(1-\gamma_{5}\right)-2 s_{W}^{2} Q_{f}\right)$} \\
\hline $\bar{U} / \bar{D}$ & $D / U$ & $W^{+} / W^{-}$ & $e \frac{1}{2 \sqrt{2} s_{W}} \gamma^{\mu}\left(1-\gamma_{5}\right)$ \\
\hline
\end{tabular}




\section{B.10 Fermion-Fermion-Scalar}

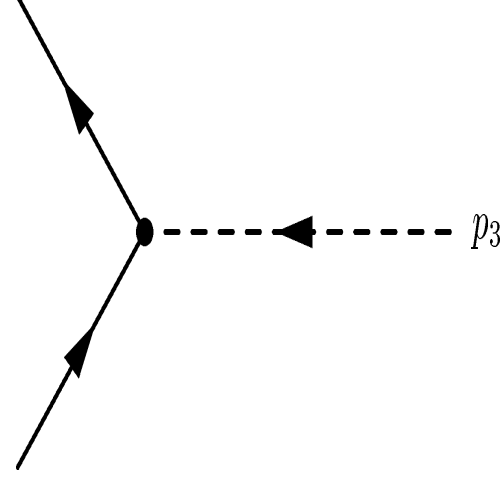

\begin{tabular}{cccc}
\hline$p_{1}$ & $p_{2}$ & $p_{3}$ \\
\hline $\bar{f}$ & $f$ & $H$ & $-e \frac{1}{2 s_{W}} \frac{m_{f}}{M_{W}}$ \\
\hline $\bar{U} / \bar{D}$ & $U / D$ & $\chi_{3}$ & $(-/+) i e \frac{1}{2 s_{W}} \frac{m_{f}}{M_{W}} \gamma_{5}$ \\
\hline $\bar{U}$ & $D$ & $\chi^{+}$ \\
$-i e \frac{1}{2 \sqrt{2} s_{W}} \frac{1}{M_{W}}\left[\left(m_{D}-m_{U}\right)+\left(m_{D}+m_{U}\right) \gamma_{5}\right]$ \\
\hline $\bar{D}$ & $U$ & $\chi^{-}$ \\
$-i e \frac{1}{2 \sqrt{2} s_{W}} \frac{1}{M_{W}}\left[\left(m_{U}-m_{D}\right)+\left(m_{U}+m_{D}\right) \gamma_{5}\right]$ \\
\hline
\end{tabular}

\section{B.11 Ghost-Ghost-Vector}

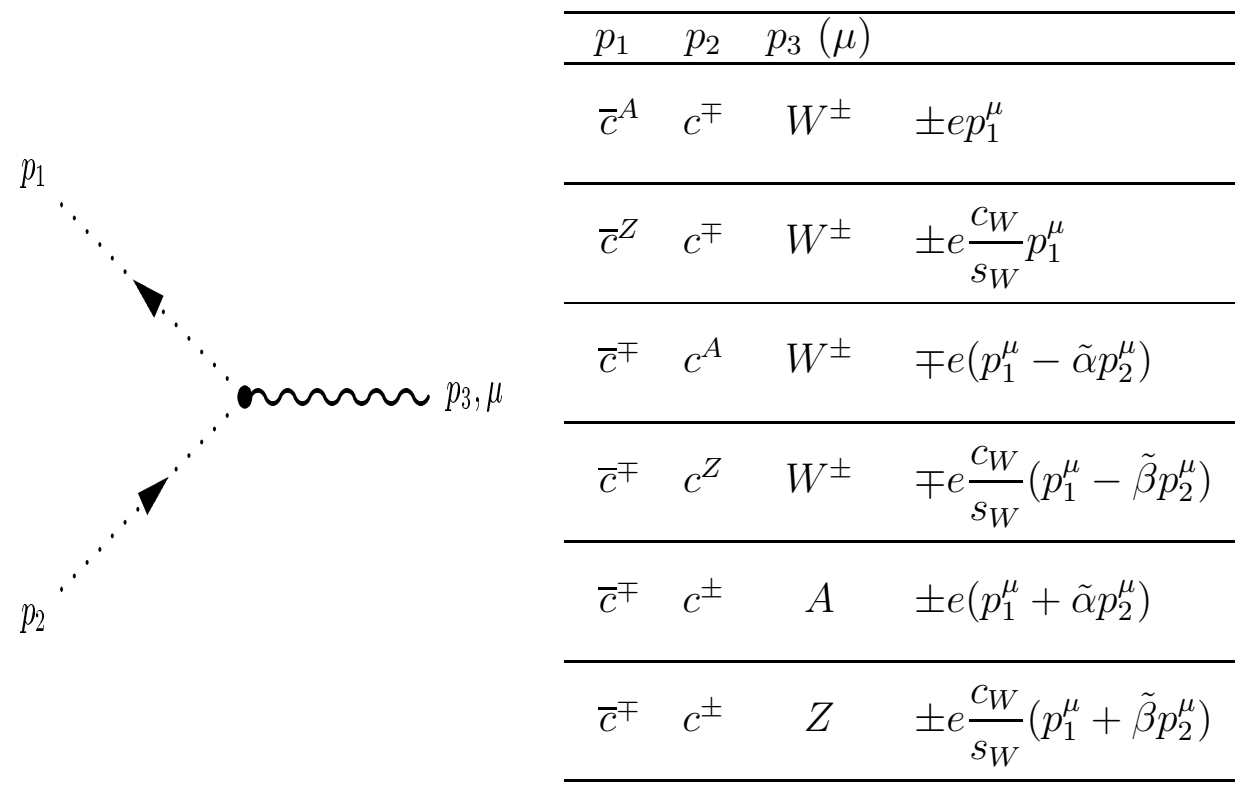




\section{B.12 Ghost-Ghost-Scalar}

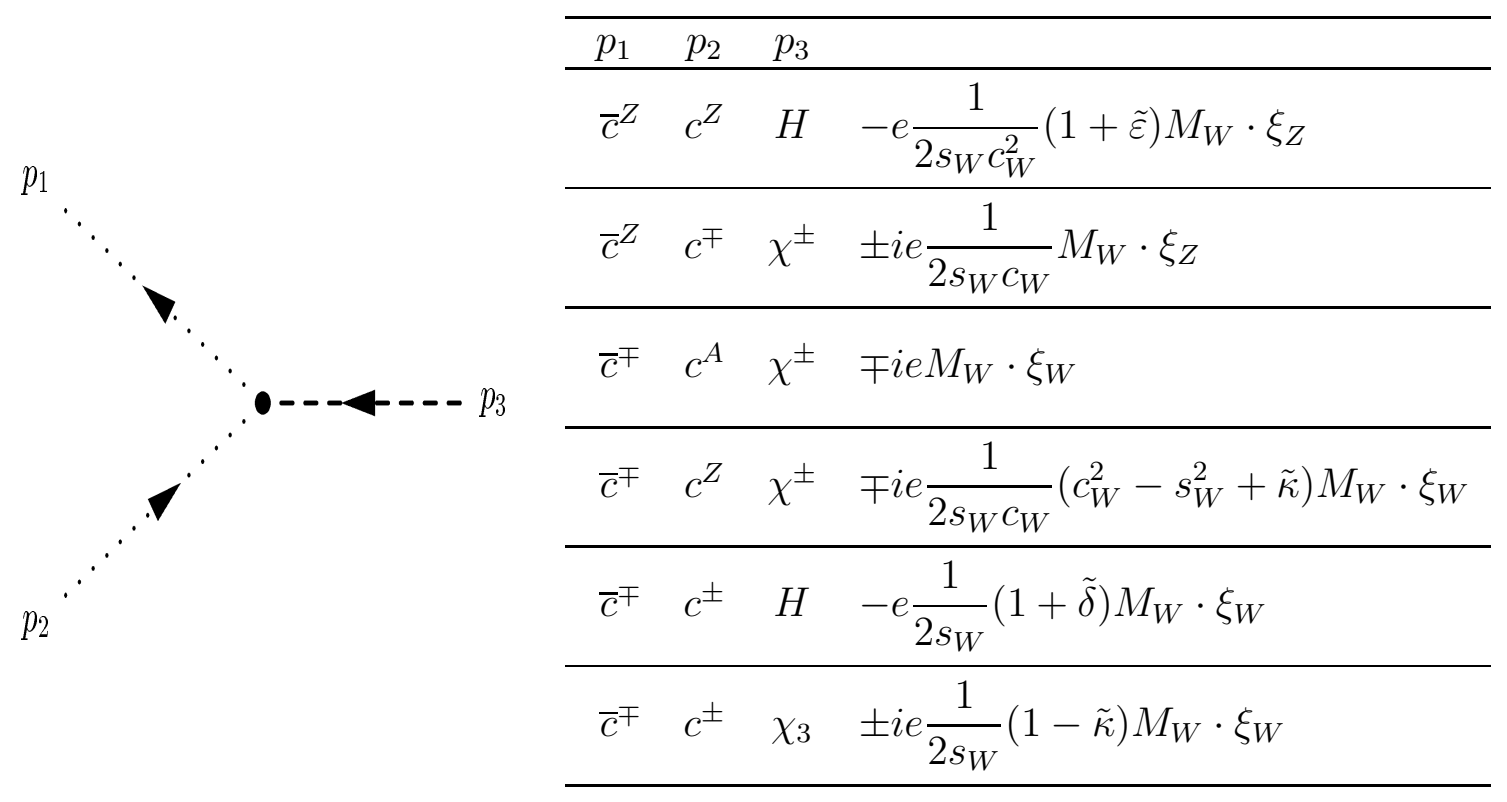




\section{B.13 Ghost-Ghost-Vector-Vector}

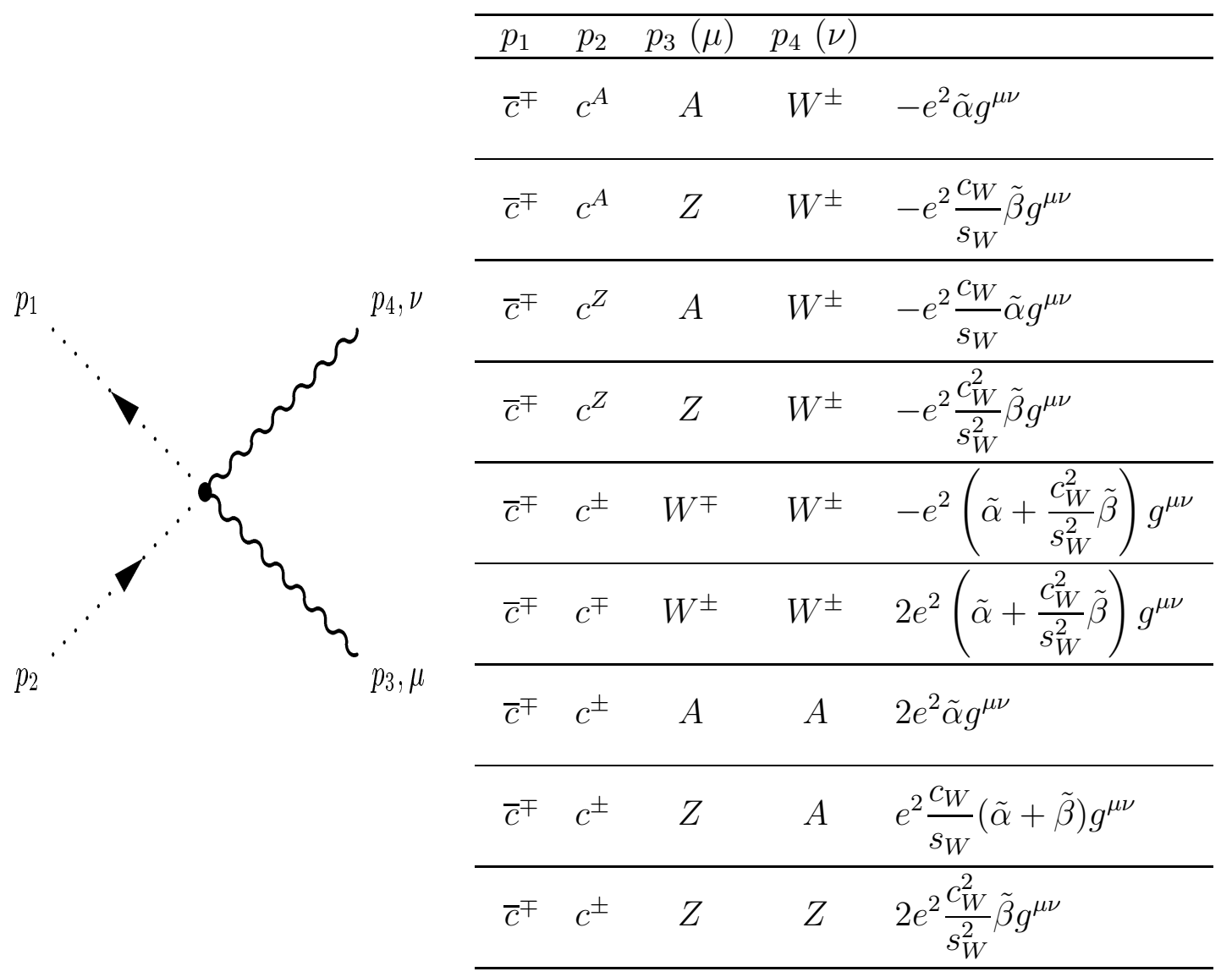




\section{B.14 Ghost-Ghost-Scalar-Scalar}

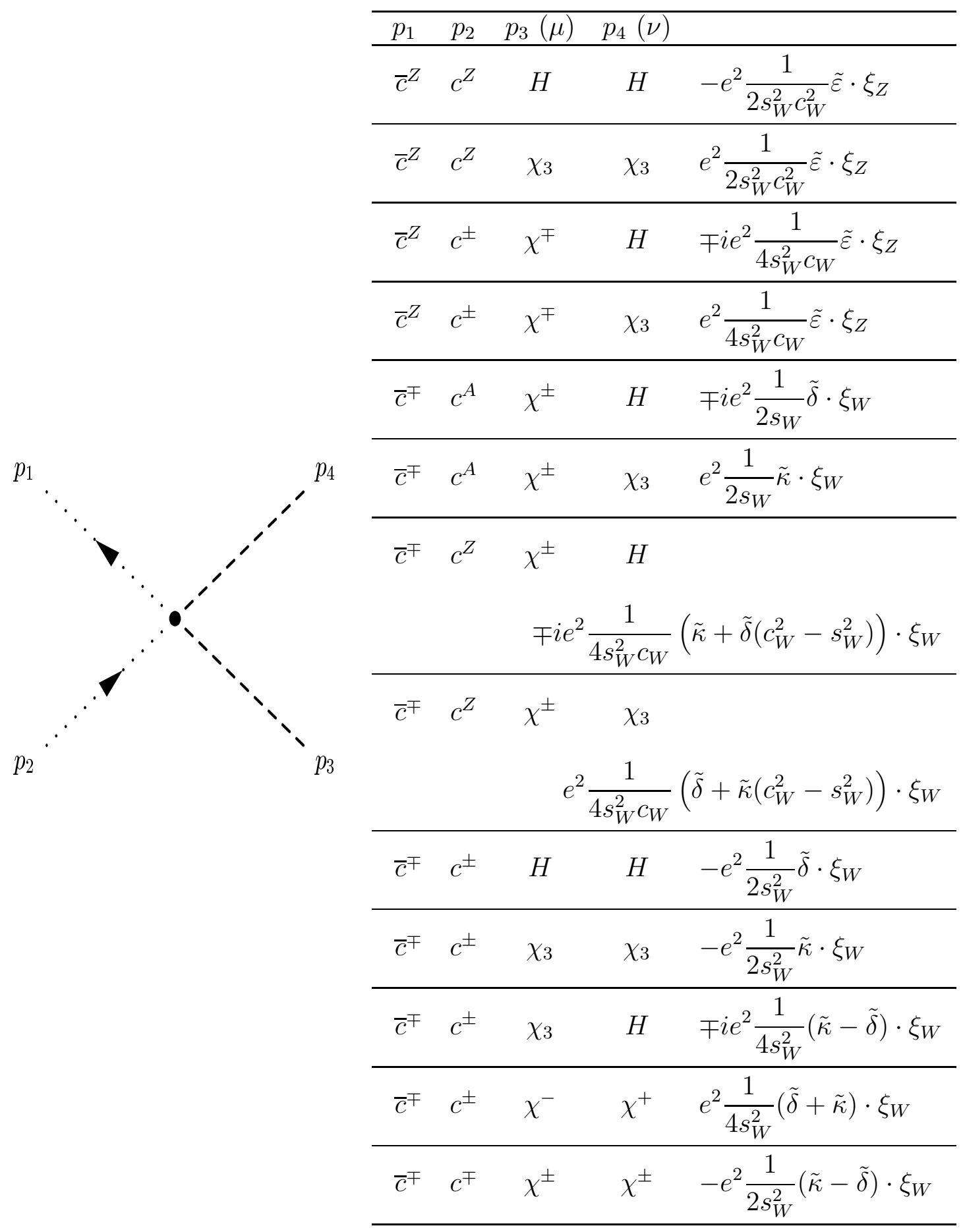




\section{Counterterms in the ghost sector}

Since we deal specifically with processes at one-loop, there is no need to dwell on the renormalisation of the ghost sector. We only briefly sketch the procedure without giving explicit formulae for the various counterterms and the renormalisation constants.

There is some freedom for the introduction of renormalisation constants for the ghost fields. We use the following convention.

$$
\begin{aligned}
\underline{c}^{ \pm} & =\tilde{Z}_{3} c^{ \pm} \\
\left(\begin{array}{c}
\underline{c}^{Z} \\
\underline{c}^{A}
\end{array}\right) & =\left(\begin{array}{ll}
\tilde{Z}_{Z Z} & \tilde{Z}_{Z A} \\
\tilde{Z}_{A Z} & \tilde{Z}_{A A}
\end{array}\right)\left(\begin{array}{l}
c^{Z} \\
c_{A}
\end{array}\right) \\
\underline{\bar{c}}^{ \pm} & =\bar{c}^{ \pm} \\
\overline{\bar{c}}^{Z} & =\bar{c}^{Z} \\
\underline{\bar{c}}^{A} & =\bar{c}^{A}
\end{aligned}
$$

To derive the full counterterm Lagrangian for the ghost we appeal to the auxiliary $B$-field formulation of the gauge-fixing Lagrangian $\mathcal{L}_{G F}$, see Eq. 5.17 in section [5.2. As stressed previously $\mathcal{L}_{G F}$ is written in terms of renormalised fields and as such does not induce any counterterm. However the BRST transformation are defined for bare fields. Therefore in order to generate the ghost Lagrangian including counterterms one needs to re-express $\mathcal{L}_{G F}$ in terms of bare fields to first generate $\mathcal{L}_{G h}$ with bare fields. From there one can then derive the counterterm ghost Lagrangian. One exploits the freedom in the renormalisation of the $B$-fields so that the combination of the $B$ fields and gauge fields shows no explicit dependence on wave function renormalisation. We therefore define

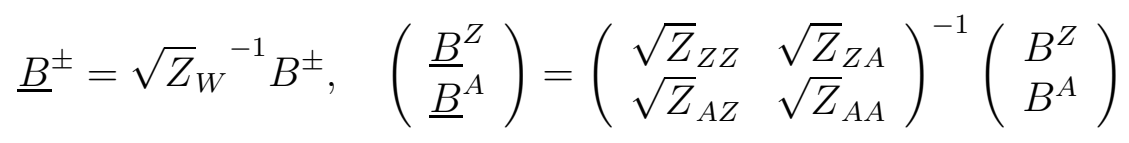

The relation is just the inverse of that for gauge fields.

Then Eq. 5.17] is

$$
\begin{aligned}
\mathcal{L}_{G F}= & \underline{B}^{+} \partial^{\mu} \underline{W}_{\mu}^{-}+B^{+} \xi_{W} M_{W} \chi^{-}+(\text {h.c. }) \\
& +\underline{B}^{Z} \underline{\partial}^{\mu} \underline{Z}_{\mu}+B^{Z} \xi_{Z} M_{Z} \chi_{3}+\underline{B}{ }^{A} \partial \underline{A}_{\mu} \\
& + \text { non-linear gauge terms }(\tilde{\alpha}, \tilde{\beta}, \tilde{\delta}, \tilde{\epsilon}, \tilde{\kappa}) \\
& +\underline{B} \text {-linear terms. }
\end{aligned}
$$

One then makes the identifications

$$
\begin{gathered}
B^{+} \xi_{W} M_{W} \chi^{-}=\underline{B}^{+} \underline{\xi}_{W} \underline{M}_{W} \underline{\chi}^{-}, \\
B^{Z} \xi_{Z} M_{Z} \chi_{3}=\underline{B}^{Z} \underline{\xi}_{Z} \underline{M}_{Z} \underline{\chi}_{3}+\underline{B}^{A} \underline{\xi}_{Z A} \underline{M}_{Z} \underline{\chi}_{3}
\end{gathered}
$$


where we defined the renormalisation of the gauge parameters as

$$
\begin{aligned}
& \underline{\xi}_{W}=\xi_{W}\left(M_{W} / \underline{M}_{W}\right) \sqrt{Z}_{W} \sqrt{Z}_{\chi}^{-1} \\
& \underline{\xi}_{Z}=\xi_{Z}\left(M_{Z} / \underline{M}_{Z}\right) \sqrt{Z}_{Z Z} \sqrt{Z}_{\chi_{3}}^{-1} \\
& \underline{\xi}_{Z A}=\xi_{Z}\left(M_{Z} / \underline{M}_{Z}\right) \sqrt{Z}_{Z A} \sqrt{Z}_{\chi_{3}}^{-1}
\end{aligned}
$$

$\underline{\xi}_{Z A}$ is not an independent parameter but the short-hand notation given by Eq. C.6. Non-linear gauge terms can be transformed in a similar way by the renormalisation of $(\tilde{\alpha}, \tilde{\beta}, \tilde{\delta}, \tilde{\epsilon}, \tilde{\kappa})$. Note that the terms bilinear in the $B$ fields would get extra factors. However this does not affect the renormalisation program since $\delta_{\mathrm{BRS}} B=0$. This helps define the bare $G$ functions.

We obtain bare $G$ terms by the above equations.

$$
\begin{aligned}
& \underline{G}^{\mp}=\partial^{\mu} \underline{W}_{\mu}^{\mp}+\underline{\xi}_{W} \underline{M}_{W} \underline{\chi}^{\mp}+\text { non }- \text { linear gauge terms } \\
& \underline{G}^{Z}=\partial^{\mu} \underline{Z}_{\mu}+\underline{\xi}_{Z} \underline{M}_{Z} \underline{\chi}_{3}+\text { non }- \text { linear gauge terms } \\
& \underline{G}^{A}=\partial^{\mu} \underline{A}_{\mu}+\underline{\xi}_{Z A} \underline{M}_{Z} \underline{\chi}_{3}+\text { non }- \text { linear gauge terms }
\end{aligned}
$$

Except for $G^{A}$, these are the same as those in 5.15 assuming that quantities are bare ones. With these, one defines the bare ghost Lagrangian, that contains extra terms than those obtained at tree-level due to the induced mixing $\xi_{Z A}$. One then readily obtains the renormalised ghost Lagrangian.

\section{Auxiliary Fields and Generalised Ward-Takahashi Identities in the unphysical scalar sector}

Although the renormalisation of this sector is not essential if one wants to arrive at finite S-matrix elements, the various two-point functions of the Goldstones and the longitudinal gauge bosons as well as their mixing are related.

To easily derive these generalised Ward-Takahashi identities that constrain the different propagators it is very useful to introduce $\mathcal{L}_{G F}$ via the auxiliary fields as done in Eq. 5.17. The Ward-Takahashi identities are particularly easy to derive if one works with the $B$-fields and considers the BRST transformations on some specific Green's functions (vacuum expectation values of time ordered products). For the two-point function of any two fields $\phi_{A}$ and $\phi_{B}$, we use the short-hand notation:

$$
\left\langle\phi_{A} \phi_{B}\right\rangle=\left\langle 0\left|\left(T \phi_{A}(x) \phi_{B}(y)\right)\right| 0\right\rangle
$$

For example, take the generic Green's function $\left\langle\bar{c}^{i} B^{j}\right\rangle$ which in fact is zero (it has a non vanishing ghost number). Subjecting it to a BRST transformation one gets: 


$$
\begin{aligned}
\delta_{\mathrm{BRS}}\left\langle\bar{c}^{i} B^{j}\right\rangle & =\left\langle\left(\delta_{\mathrm{BRS}} \bar{c}^{i}\right) B^{j}\right\rangle-\left\langle\bar{c}^{i}\left(\delta_{\mathrm{BRS}} B^{j}\right)\right\rangle=i\left\langle B^{i} B^{j}\right\rangle=0 \\
& \text { or }\left\langle G^{i} G^{j}\right\rangle=0
\end{aligned}
$$

where in the last part we have used the equation of motion for the $B^{i}$ s. The above relation leads directly to a constraint on the two-point functions of the gauge vector boson, the gauge-Goldstone mixing and the Goldstone two-point functions. One novelty compared to the usual linear gauge is that these identities also involve correlation functions with composite operators. Indeed if we specialise to the $Z Z$ functions, one has with $\xi_{Z}=1$

$$
\begin{aligned}
& \left\langle G_{Z}(x) G_{Z}(y)\right\rangle= \\
\langle & \left(\left(\partial . Z(x)+M_{Z} \chi_{3}(x)+\frac{g}{2 c_{W}} \tilde{\epsilon} H(x) \chi_{3}(x)\right)\left(\left(\partial . Z(y)+M_{Z} \chi_{3}(y)+\frac{g}{2 c_{W}} \tilde{\epsilon} H(y) \chi_{3}(y)\right)\right\rangle\right. \\
= & \partial_{x}^{\mu} \partial_{y}^{\nu}\left\langle Z_{\mu}(x) Z_{\nu}(y)\right\rangle+2 M_{Z} \partial_{x}^{\mu}\left\langle Z_{\mu}(x) \chi_{3}(y)\right\rangle+M_{Z}^{2}\left\langle\chi_{3}(x) \chi_{3}(y)\right\rangle \\
+ & \tilde{\epsilon} \frac{g}{c_{W}}\left[\partial_{x}^{\mu}\left\langle Z_{\mu}(x)\left(H(y) \chi_{3}(y)\right)\right\rangle+M_{Z}\left\langle\chi_{3}(x)\left(H(y) \chi_{3}(y)\right)\right\rangle\right] \\
+ & \left(\frac{g \tilde{\epsilon}}{2 c_{W}}\right)^{2}\left\langle H(x) \chi_{3}(x) H(y) \chi_{3}(y)\right\rangle=0 .
\end{aligned}
$$

It is important to realise that these are the full Green's function and therefore the external legs are not amputated. Therefore it is crucial to note that the last two terms (in the last two lines) do not have the double pole structure.

This translates into the following identity, in momentum space,

$$
\left(q^{2}\left(\Pi_{L}^{Z Z}-2 M_{Z} \Pi^{Z \chi_{3}}\right)+M_{Z}^{2} \Pi^{\chi_{3} \chi_{3}}\right) \equiv A_{Z Z}
$$

Note that in the approach where the gauge-fixing Lagrangian is expressed in terms of renormalised quantities, Eq. D.11 also holds for the renormalised two-point function. In the linear gauge $A_{Z Z}=0$ for any $q^{2}$ (tadpole contributions must be included here). An explicit calculation gives

$$
\begin{aligned}
A_{Z Z}= & \frac{\alpha \tilde{\epsilon}}{16 \pi s_{W}^{2} c_{W}^{2}}\left(q^{2}-M_{Z}^{2}\right)\left\{\tilde{\epsilon}\left(q^{2}-3 M_{Z}^{2}\right)\left(C_{U V}-F_{0}(Z H)\right)\right. \\
& \left.+2\left[q^{2}\left(F_{0}(Z H)-2 F_{1}(Z H)\right)-M_{H}^{2}\left(C_{U V}-F_{0}(Z H)\right)\right]\right\} .
\end{aligned}
$$

The functions $F_{0,1}$ are defined in Eq. G.8. We see clearly that $A_{Z Z}$ does not vanish in the non-linear gauge, but at the $Z$ pole. In fact the contribution $A_{Z Z}$ can be derived directly from the last two terms of Eq. D.10. In a diagrammatic form, at one-loop, Eq. D.10 can be described as 


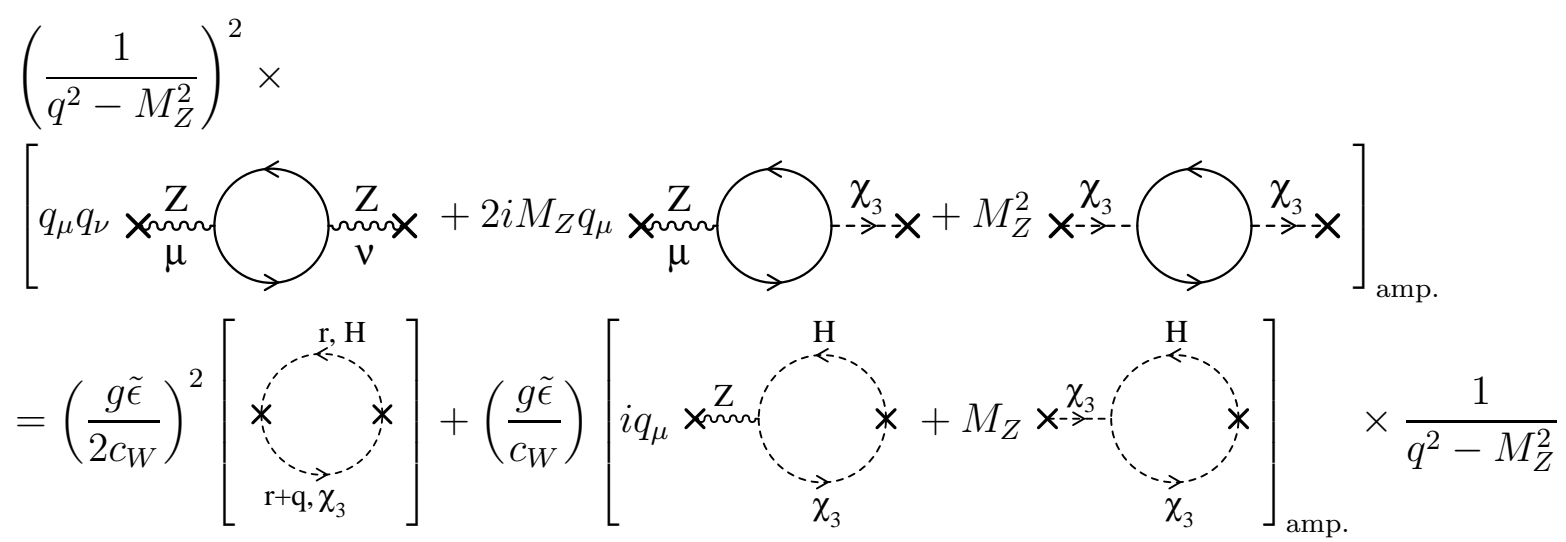

Calculating the new graphs explicitly confirms the identity and is a check on our calculation of $\Pi_{L}^{Z Z}, \Pi^{Z \chi_{3}}$ and $\Pi^{\chi_{3} \chi_{3}}$. Note that for the genuine two-point functions (contributing to $A_{Z Z}$ ) the loops include all possible particles including matter fields. For the latter one gets the same contribution as in the linear gauge.

For the charged sector the identities go along the same line.

For the photon, the identities give

$$
\Pi_{L}^{A A}=0 \quad \text { at any } q^{2} .
$$

For the $A Z$ transition we get (from $\left\langle G_{A} G_{Z}\right\rangle=0$ ) that

$$
\Pi_{L}^{A Z}-M_{Z} \Pi^{A \chi_{3}}=0 \text {. }
$$

This identity holds at any $q^{2}$, but only at one-loop thanks to the fact that the vertex $A H \chi_{3}$ does not exist at tree-level. This can be easily checked by looking up the explicit formulae in sections $\mathrm{H.2}$ and $\mathrm{H.3}$ and section $\mathrm{H.8.1}$.

\section{E Renormalising the gauge-fixing functions}

So far we have chosen to take the gauge-fixing term as being written in terms of renormalised quantities. Although this is quite practical and avoids the introduction of more counterterms for the (unphysical) parameters entering the gauge-fixing Lagrangian, it does not lead to finite Green's functions, in the general case of the non-linear gauge, even when all Feynman parameters are set to one, $\xi_{A, Z, W}=1$. However all S-matrix elements are finite and gauge-parameter independent. Therefore as we argued, this approach of taking the gauge-fixing Lagrangian as renormalised from the outset is easy to implement and at the same time acts as a good test on our system since many divergences in a few Green's 
functions cancel at the level of the S-matrix. If one wants to have finite Green's functions one also needs to consider counterterms to the gauge-fixing Lagrangian, Eq. 15.15, The purpose of this Appendix is to show how all two-point functions can be made finite if one also introduces counterterms for the gauge parameters, beside the renormalisation of the physical parameters (masses, electric charge) and the tadpole as well as the wave-function renormalisation for all fields as defined in the main text, see section 6.1. For the two-point functions, the difference between this approach (taking a bare gauge fixing Lagrangian) and that of the paper (taking the gauge fixing as renormalised), only concerns the unphysical scalars (and longitudinal part of the vector bosons). Instead of Eq. 5.15 we write for the charged sector the gauge fixing terms in bare quantities,

$$
\mathcal{L}_{G F, W}=-\frac{1}{\underline{\xi_{W}}} \mid\left(\partial_{\mu}-i \underline{\underline{e}} \underline{\tilde{\alpha}} \underline{A}_{\mu}-i \underline{g} \underline{c_{W}} \underline{\tilde{\beta}} \underline{Z}_{\mu}\right) \underline{W}^{\mu+}+\underline{\xi}_{W}^{\prime} \frac{\underline{g}}{2}\left(\left.\left(\underline{M}_{W}+\frac{g}{2}\left(\underline{\tilde{\delta}} \underline{H}+i \underline{\tilde{\kappa}} \underline{\chi}_{3}\right)\right) \underline{\chi}^{+}\right|^{2} .\right.
$$

For the neutral sector one has to allow for $A-Z$ and $A-\chi_{3}$ mixing. We take ${ }^{14}$

$$
\begin{aligned}
\mathcal{L}_{G F,(Z, A)}=- & \frac{1}{2 \underline{\xi_{Z}}}\left(\partial \cdot \underline{Z}+\underline{\xi_{Z}^{\prime}}\left(\underline{M_{Z}}+\frac{\underline{g}}{2 \underline{c_{W}}} \underline{\tilde{\varepsilon}} \underline{H}\right) \underline{\chi_{3}}\right)^{2}-\frac{1}{2 \underline{\xi_{A}}}\left(\partial \cdot \underline{A}+\delta \xi_{A}^{\prime} M_{Z} \underline{\chi_{3}}\right)^{2} \\
& +\delta \eta_{A Z} \partial \cdot \underline{A} \partial \cdot \underline{Z} .
\end{aligned}
$$

It will also be useful to introduce $\underline{\tilde{\xi}_{W}}$ and $\underline{\tilde{\xi}_{Z}}$ such that $\underline{\xi_{W}^{\prime}}=\sqrt{\underline{\xi_{W}} \frac{\tilde{\xi}_{W}}{\xi^{\prime}}}, \underline{\xi_{Z}^{\prime}}=\sqrt{\underline{\xi_{Z}}} \underline{\tilde{\xi}_{Z}}$. At tree-level our implementation requires that $\underline{\xi}_{A, Z, W}=\underline{\xi}_{Z, W}^{\prime}=1, \overline{\delta \xi_{A}^{\prime}}=\delta \overline{\eta_{A Z}}=0 . \delta \overline{\xi_{A}^{\prime}}$ and $\delta \eta_{A Z}$ should be considered of order $\mathcal{O}(\alpha)$ and are introduced to avoid that $\mathcal{L}_{G F,(Z, A)}$ does not induce any non diagonal photon transition. Apart from the $\delta \xi_{A}^{\prime}$ and $\delta \eta_{A Z}$ terms, these bare gauge fixing conditions are, of course, formally the same as those we introduced in Eq. 5.15. In fact since the gauge fixing for the photon is still linear and that we are working with $\xi_{A}=1$, we could still take the gauge-fixing for the photon to be renormalised. As we will see later, the two approaches lead to the same form of the two-point functions for $\hat{\Pi}_{L}^{A A, A Z}$ and $\hat{\Pi}^{A \chi_{3}}$. Since we only seek to show how finite two-point functions can be arrived at, it is sufficient to only consider the addition of the counterterms to the Feynman gauge parameters $\xi$ and $\xi^{\prime}$. The bare and renormalised Feynman gauge parameters are related as

$$
\underline{\xi_{i}}=\xi_{i}+\delta \xi_{i} ; \quad \underline{\xi_{i}^{\prime}}=\xi_{i}^{\prime}+\delta \xi_{i}^{\prime} ; \quad i=W, Z, A .
$$

\footnotetext{
${ }^{14} \mathcal{L}_{G F,(Z, A)}$ be derived through the auxiliary B-field formulation and the gauge functions $G^{A}$ and $G^{Z}$. One writes $\mathcal{L}_{G F,(Z, A)}=\left(\underline{\xi_{Z}} / 2\right)\left(\underline{B}^{Z}\right)^{2}+\left(\underline{\xi_{A}} / 2\right)\left(\underline{B}^{A}\right)^{2}+\delta \tilde{\eta}_{A Z} \underline{B}^{A} \underline{B}^{Z}+\underline{B}^{Z} \underline{G}^{Z}+\underline{B}^{A} \underline{G}^{A}$ with $\underline{G}^{Z}=\partial . \underline{Z}+$ $\underline{\xi_{Z}^{\prime}}\left(\underline{M_{Z}}+\left(\underline{g} / 2 \underline{c_{W}}\right) \underline{\tilde{\varepsilon}} \underline{H}\right) \underline{\chi}_{3}$ and $\underline{G}^{A}=\partial \cdot \underline{A}+\delta \xi_{A \chi}^{\prime} \underline{M_{Z}} \chi_{3}$. For the purpose of generating the counterterms for the two-point functions at the one-loop order, one makes the identification, $\delta \eta_{A Z}=\delta \tilde{\eta}_{A Z} /\left(\xi_{A} \xi_{Z}\right)$ and $\delta \xi_{A}^{\prime}=\delta \xi_{A \chi}-\left(\xi_{Z}^{\prime} / \xi_{Z}\right) \delta \tilde{\eta}_{A Z}$.
} 
Since we will only work in the 't Hooft-Feynman gauge, it is sufficient to only consider the case with all $\xi_{i}=\xi_{i}^{\prime}=1$ apart from $\xi_{A}^{\prime}=\delta \eta_{A Z}=0$.

The generated counterterms for the two-point functions, allowing for the renormalisation of the gauge fixing Lagrangian, and for the approach we take in GRACE-1oop of considering the gauge fixing Lagrangian in Eq. 5.15 renormalised, are shown below.

1. Vector-Vector

\begin{tabular}{l||l|l} 
& $\mathcal{L}_{G F}$ with renormalised quantities & $\mathcal{L}_{G F}$ with bare quantities \\
\hline$W W$ & $\hat{\Pi}_{T}^{W}=\delta M_{W}^{2}+2\left(M_{W}^{2}-q^{2}\right) \delta Z_{W}^{1 / 2}$ & unchanged \\
& $\hat{\Pi}_{L}^{W}=\delta M_{W}^{2}+2 M_{W}^{2} \delta Z_{W}^{1 / 2}$ & $\hat{\Pi}_{L}^{W}=2\left(M_{W}^{2}-q^{2}\right) \delta Z_{W}^{1 / 2}+\delta M_{W}^{2}+q^{2} \delta \xi_{W}$ \\
\hline$Z Z$ & $\hat{\Pi}_{T}^{Z Z}=\delta M_{Z}^{2}+2\left(M_{Z}^{2}-q^{2}\right) \delta Z_{Z Z}^{1 / 2}$ & unchanged \\
& $\hat{\Pi}_{L}^{Z Z}=\delta M_{Z}^{2}+2 M_{Z}^{2} \delta Z_{Z Z}^{1 / 2}$ & $\hat{\Pi}_{L}^{Z Z}=2\left(M_{Z}^{2}-q^{2}\right) \delta Z_{Z Z}^{1 / 2}+\delta M_{Z}^{2}+q^{2} \delta \xi_{Z}$ \\
\hline$Z A$ & $\hat{\Pi}_{T}^{Z A}=\left(M_{Z}^{2}-q^{2}\right) \delta Z_{Z A}^{1 / 2}-q^{2} \delta Z_{A Z}^{1 / 2}$ & unchanged \\
& $\hat{\Pi}_{L}^{Z A}=M_{Z}^{2} \delta Z_{Z A}^{1 / 2}$ & $M_{Z}^{2} \delta Z_{Z A}^{1 / 2}-q^{2}\left(\delta Z_{Z A}^{1 / 2}+\delta Z_{A Z}^{1 / 2}-\delta \eta_{A Z}\right)$ \\
\hline$A A$ & $\hat{\Pi}_{T}^{A A}=-2 q^{2} \delta Z_{A A}^{1 / 2}$ & unchanged \\
& $\hat{\Pi}_{L}^{A A}=0$ & $\hat{\Pi}_{L}^{A A}=-2 q^{2} \delta Z_{A A}^{1 / 2}+q^{2} \delta \xi_{A}$ \\
\hline
\end{tabular}

2. Scalar-Scalar

\begin{tabular}{c||l|l} 
& $\mathcal{L}_{G F}$ with renormalised quantities & $\mathcal{L}_{G F}$ with bare quantities \\
\hline$H H$ & $\hat{\Pi}^{H}=2\left(q^{2}-M_{H}^{2}\right) \delta Z_{H}^{1 / 2}-\delta M_{H}^{2}+\frac{3 \delta T}{v}$ & unchanged \\
\hline$\chi_{3} \chi_{3}$ & $\hat{\Pi}^{\chi_{3}}=2 q^{2} \delta Z_{\chi_{3}}^{1 / 2}+\frac{\delta T}{v}$ & $\hat{\Pi}^{\chi_{3}}=2\left(q^{2}-M_{Z}^{2}\right) \delta Z_{\chi_{3}}^{1 / 2}-M_{Z}^{2} \delta \tilde{\xi}_{Z}$ \\
& & $-\delta M_{Z}^{2}+\frac{\delta T}{v}$ \\
\hline$\chi \chi$ & $\hat{\Pi}^{\chi}=2 q^{2} \delta Z_{\chi}^{1 / 2}+\frac{\delta T}{v}$ & $\hat{\Pi}^{\chi}=2\left(q^{2}-M_{W}^{2}\right) \delta Z_{\chi}^{1 / 2}-M_{W}^{2} \delta \tilde{\xi}_{W}$ \\
& & $-\delta M_{W}^{2}+\frac{\delta T}{v}$ \\
\hline
\end{tabular}

3. Vector-Scalar

\begin{tabular}{l||l|l} 
& $\mathcal{L}_{G F}$ with renormalised quantities & $\mathcal{L}_{G F}$ with bare quantities \\
\hline$W \chi$ & $\hat{\Pi}^{W \chi}=M_{W}\left(\delta G_{W}+\delta Z_{W}^{1 / 2}+\delta Z_{\chi}^{1 / 2}\right)$ & $\hat{\Pi}^{W \chi}=\frac{M_{W}}{2}\left(\delta \xi_{W}-\delta \tilde{\xi}_{W}\right)$ \\
\hline$Z \chi_{3}$ & $\hat{\Pi}^{Z \chi_{3}}=M_{Z}\left(\delta G_{Z}+\delta Z_{Z Z}^{1 / 2}+\delta Z_{\chi_{3}}^{1 / 2}\right)$ & $\hat{\Pi}^{Z \chi_{3}}=\frac{M_{Z}}{2}\left(\delta \xi_{Z}-\delta \tilde{\xi}_{Z}\right)$ \\
\hline$A \chi_{3}$ & $\hat{\Pi}^{A \chi_{3}}=M_{Z} \delta Z_{Z A}^{1 / 2}$ & $\hat{\Pi}^{A \chi_{3}}=-M_{Z} \delta \xi_{A}^{\prime}$ \\
\hline
\end{tabular}




\section{Fermion-Fermion}

This remains the same in both approaches and is given by Eq. 6.6.

Note that in both approaches one has $\hat{\Pi}_{L}^{V}(0)=\hat{\Pi}_{T}^{V}(0)$ for all vectors as should be. Note also that for the 2-point functions involving photons, $\hat{\Pi}_{L}^{A A, Z A}$ and $\hat{\Pi}^{A \chi_{3}}, \delta \xi_{A}, \delta \xi_{A}^{\prime}$ and $\delta \eta_{A Z}$ can be chosen so that the counterterms in both approaches are the same. In particular the Ward identities $\Pi_{L}^{A A}=0$ and $\Pi_{L}^{A Z}-M_{Z} \Pi^{A \chi_{3}}=0$, see Eqs. D.14 D.15, are maintained after renormalisation. Therefore we can take

$$
\begin{aligned}
\delta \xi_{A} & =2 \delta Z_{A A}^{1 / 2}, \quad \delta \xi_{A}^{\prime}=-\delta Z_{Z A}^{1 / 2} \\
\delta \eta_{A Z} & =\delta Z_{Z A}^{1 / 2}+\delta Z_{A Z}^{1 / 2} .
\end{aligned}
$$

Let us turn to the charged sector (the $Z$ transitions go along the same line). Defining

$$
A_{W W}^{C T}=q^{2}\left(\hat{\Pi}_{L}^{W}-2 M_{W} \hat{\Pi}^{W \chi}\right)+M_{W}^{2} \hat{\Pi}^{\chi \chi}
$$

we find that

$$
\begin{aligned}
A_{W W}^{C T}= & 0 \text { in our approach (as expected) } \\
= & \left(q^{2}-M_{W}^{2}\right)\left(q^{2} \delta \xi_{W}+M_{W}^{2} \delta \tilde{\xi}_{W}+\delta M_{W}^{2}+2 M_{W}^{2} \delta Z_{\chi}^{1 / 2}-2 q^{2} \delta Z_{W}^{1 / 2}\right) \\
& \text { with } \mathcal{L}_{G F} \text { in terms of bare fields. }
\end{aligned}
$$

This again means that there are constraints on $\xi_{W}$ and $\tilde{\xi}_{W}$, i.e they are not independent once the other wave functions have been set. Exactly the same applies for the $Z Z$ transition. Also this means that on-shell renormalisation for the unphysical sector is possible. That is, that the scalars have poles at the same location as the physical vector bosons. For example for the $W$, this condition $\left(\tilde{\Pi}_{L}\left(M_{W}^{2}\right)=\tilde{\Pi}^{\chi}\left(M_{W}^{2}\right)=0\right)$ gives that

$$
\begin{aligned}
& M_{W}^{2} \delta \xi_{W}+\delta M_{W}^{2}=-\Pi_{L}^{W}\left(M_{W}^{2}\right) \\
& M_{W}^{2} \delta \tilde{\xi}_{W}+\delta M_{W}^{2}=+\Pi^{\chi}\left(M_{W}^{2}\right)+\frac{\delta T}{v}
\end{aligned}
$$

This shows that the renormalised $W-\chi$ transition becomes finite in the non-linear gauge:

$$
\tilde{\Pi}^{W \chi}=\Pi^{W \chi}+\frac{M_{W}}{2}\left(\delta \xi_{W}-\delta \tilde{\xi}_{W}\right)=\Pi^{W \chi}-\frac{1}{2 M_{W}}\left(\Pi_{L}^{W}\left(M_{W}^{2}\right)+\Pi^{\chi}\left(M_{W}^{2}\right)+\frac{\delta T}{v}\right)=\text { finite } .
$$


This can be explicitly shown by using the full expressions for the two-point functions at one-loop given in Eqs. H.14H.26 and H.32.

Using Eq. E.7, the renormalised $\chi \chi$ writes

$$
\tilde{\Pi}^{\chi}=2\left(q^{2}-M_{W}^{2}\right)\left(\delta Z_{\chi}^{1 / 2}+\frac{\alpha}{16 \pi s_{W}^{2}}\left(\tilde{\kappa}+\tilde{\delta}-\left(2+1 / c_{W}^{2}\right)\right) C_{U V}+\text { finite }\right) .
$$

In our approach we define $\delta Z_{\chi}^{1 / 2}$ so that all $C_{U V}$ terms proportional to $q^{2}$ vanish:

$$
\delta Z_{\chi}^{1 / 2} \equiv-\frac{\Pi_{C_{U V}-\text { part }}^{\chi}}{2 q^{2}}=\frac{\alpha}{16 \pi s_{W}^{2}}\left(\left(2+1 / c_{W}^{2}\right)-\tilde{\kappa}-\tilde{\delta}\right) C_{U V}
$$

This is exactly the same result had we required $\tilde{\Pi}^{\chi}$ in Eq. E.9 to be finite. This result would have been arrived at directly had we required that the residue at the pole of the

$\chi \chi$ propagator be 1 . The $C_{U V}$ part of $\delta Z_{\chi}^{1 / 2}$ would be the same, differences would appear in finite terms that have no incidence on $S$-matrix.

Having constrained $\delta \tilde{\xi}_{W}$ and $\delta Z_{\chi}^{1 / 2}, \delta \xi_{W}$ is fixed. Taking for example only the $C_{U V}$ part of $\Pi_{L}^{W}$, from Eq. E.7. one has that

$$
\delta \xi_{W}=-\frac{\delta M_{W}^{2}}{M_{W}^{2}}-\frac{\Pi_{L}^{W}\left(M_{W}^{2}\right)}{M_{W}^{2}}=2 \delta Z_{W}^{1 / 2}-\frac{\alpha}{4 \pi s_{W}^{2}}\left(5 \tilde{\alpha}^{2} s_{W}^{2}+5 \tilde{\beta}^{2} c_{W}^{2}+\frac{\tilde{\delta}^{2}}{4}+\frac{\tilde{\kappa}^{2}}{4}\right) C_{U V} .
$$

We indeed find, by explicit calculations, this to be verified. Similar results hold for the other combinations of two-point functions.

\section{F A library of counterterms for the vertices}

Here, we list the full counterterms to the vertices after applying the field redefinitions. Those for the ghost vertices are not shown since they are not required at one-loop. Those for the two-point functions (propagators) and the tadpole have been discussed separately for a proper definition of the renormalisation conditions.

$\langle\cdots\rangle$ will refer to the tree-level expression of the vertex defined in Sec $\mathrm{B}$ but with $\tilde{\alpha}=$ $\tilde{\beta}=\tilde{\delta}=\tilde{\varepsilon}=\tilde{\kappa}=0$. As a result of $Z-\gamma$ mixing new vertices, denoted as (new), appear.

To help write our results in a compact form, we introduce, as is done, in 90, the following "correction" factors

$$
\begin{aligned}
\delta G_{m j} & =\frac{\delta m_{j}}{m_{j}} \\
\delta G_{H} & =\frac{\delta M_{H}^{2}}{M_{H}^{2}}
\end{aligned}
$$




$$
\begin{aligned}
\delta G_{W} & =\frac{\delta M_{W}^{2}}{2 M_{W}^{2}} \\
\delta G_{Z} & =\frac{\delta M_{Z}^{2}}{2 M_{Z}^{2}} \\
\delta H & =\frac{\delta M_{Z}^{2}-\delta M_{W}^{2}}{2\left(M_{Z}^{2}-M_{W}^{2}\right)} \\
\delta G_{1} & =\delta G_{W}-\delta H \\
\delta G_{2} & =\delta G_{Z}-\delta H \\
\delta G_{3} & =\delta G_{Z}-\delta G_{W} \\
\delta G_{4} & =\frac{2 \delta M_{W}^{2}-\delta M_{Z}^{2}}{2 M_{W}^{2}-M_{Z}^{2}}-\delta G_{W}-\delta H
\end{aligned}
$$

\section{F.1 Vector-Vector-Vector}

\begin{tabular}{cccc}
\hline$p_{1}(\mu)$ & $p_{2}(\nu)$ & $p_{3}(\rho)$ & \\
\hline$W^{-}$ & $W^{+}$ & $A$ & $\left(\delta Y+2 \delta Z_{W}^{1 / 2}+\delta Z_{A A}^{1 / 2}\right)\langle W W A\rangle+\delta Z_{Z A}^{1 / 2}\langle W W Z\rangle$ \\
\hline$W^{-}$ & $W^{+}$ & $Z$ & $\left(\delta Y+\delta G_{1}+2 \delta Z_{W}^{1 / 2}+\delta Z_{Z Z}^{1 / 2}\right)\langle W W Z\rangle+\delta Z_{A Z}^{1 / 2}\langle W W A\rangle$ \\
\hline
\end{tabular}

\section{F.2 Vector-Vector-Scalar}

\begin{tabular}{cccl}
\hline$p_{1}(\mu)$ & $p_{2}(\nu)$ & $p_{3}$ & \\
\hline$W^{ \pm}$ & $A$ & $\chi^{\mp}$ & $\left(\delta Y+\delta G_{W}+\delta Z_{W}^{1 / 2}+\delta Z_{\chi}^{1 / 2}+\delta Z_{A A}^{1 / 2}\right)\langle W A \chi\rangle+\delta Z_{Z A}^{1 / 2}\langle W Z \chi\rangle$ \\
\hline$W^{ \pm}$ & $Z$ & $\chi^{\mp}$ & $\left(\delta Y+\delta H+\delta Z_{W}^{1 / 2}+\delta Z_{\chi}^{1 / 2}+\delta Z_{Z Z}^{1 / 2}\right)\langle W Z \chi\rangle+\delta Z_{A Z}^{1 / 2}\langle W A \chi\rangle$ \\
\hline$W^{-}$ & $W^{+}$ & $H$ & $\left(\delta Y+\delta G_{2}+\delta G_{W}+2 \delta Z_{W}^{1 / 2}+\delta Z_{H}^{1 / 2}\right)\langle W W H\rangle$ \\
\hline$Z$ & $Z$ & $H$ & $\left(\delta Y+\delta G_{2}+\delta G_{3}+\delta G_{Z}+2 \delta Z_{Z Z}^{1 / 2}+\delta Z_{H}^{1 / 2}\right)\langle Z Z H\rangle$ \\
\hline$Z$ & $A$ & $H$ & $\delta Z_{Z A}^{1 / 2}\langle Z Z H\rangle \quad($ new $)$ \\
\hline
\end{tabular}

\section{F.3 Scalar-Scalar-Vector}

\begin{tabular}{cccl}
\hline$p_{1}$ & $p_{2}$ & $p_{3}(\mu)$ & \\
\hline$H$ & $\chi^{\mp}$ & $W^{ \pm}$ & $\left(\delta Y+\delta G_{2}+\delta Z_{H}^{1 / 2}+\delta Z_{\chi}^{1 / 2}+\delta Z_{W}^{1 / 2}\right)\langle H \chi W\rangle$ \\
\hline$\chi_{3}$ & $\chi^{\mp}$ & $W^{ \pm}$ & $\left(\delta Y+\delta G_{2}+\delta Z_{\chi_{3}}^{1 / 2}+\delta Z_{\chi}^{1 / 2}+\delta Z_{W}^{1 / 2}\right)\left\langle\chi_{3} \chi W\right\rangle$ \\
\hline$\chi^{-}$ & $\chi^{+}$ & $A$ & $\left(\delta Y+2 \delta Z_{\chi}^{1 / 2}+\delta Z_{A A}^{1 / 2}\right)\langle\chi \chi A\rangle+\delta Z_{Z A}^{1 / 2}\langle\chi \chi Z\rangle$ \\
\hline$\chi^{-}$ & $\chi^{+}$ & $Z$ & $\left(\delta Y+\delta G_{4}+2 \delta Z_{\chi}^{1 / 2}+\delta Z_{Z Z}^{1 / 2}\right)\langle\chi \chi Z\rangle+\delta Z_{A Z}^{1 / 2}\langle\chi \chi A\rangle$ \\
\hline$H$ & $\chi_{3}$ & $Z$ & $\left(\delta Y+\delta G_{2}+\delta G_{3}+\delta Z_{H}^{1 / 2}+\delta Z_{\chi_{3}}^{1 / 2}+\delta Z_{Z Z}^{1 / 2}\right)\left\langle H \chi_{3} Z\right\rangle$ \\
\hline$H$ & $\chi_{3}$ & $A$ & $\delta Z_{Z A}^{1 / 2}\left\langle H \chi_{3} Z\right\rangle$ (new) \\
\hline
\end{tabular}




\section{F.4 Scalar-Scalar-Scalar}

\begin{tabular}{cccc}
\hline$p_{1}$ & $p_{2}$ & $p_{3}$ & \\
\hline$H$ & $H$ & $H$ & {$\left[\left(\delta Y+\delta G_{2}-\delta G_{W}+\delta G_{H}+3 \delta Z_{H}^{1 / 2}\right)-\delta T \frac{e}{s_{W} M_{W} M_{H}^{2}}\right]\langle H H H\rangle$} \\
\hline$H$ & $\chi^{-}$ & $\chi^{+}$ & {$\left[\left(\delta Y+\delta G_{2}-\delta G_{W}+\delta G_{H}+\delta Z_{H}^{1 / 2}+2 \delta Z_{\chi}^{1 / 2}\right)-\delta T \frac{e}{s_{W} M_{W} M_{H}^{2}}\right]\langle H \chi \chi\rangle$} \\
\hline$H$ & $\chi_{3}$ & $\chi_{3}$ & {$\left[\left(\delta Y+\delta G_{2}-\delta G_{W}+\delta G_{H}+\delta Z_{H}^{1 / 2}+2 \delta Z_{\chi_{3}}^{1 / 2}\right)-\delta T \frac{e}{s_{W} M_{W} M_{H}^{2}}\right]\left\langle H \chi_{3} \chi_{3}\right\rangle$} \\
\hline
\end{tabular}

\section{F.5 Vector-Vector-Vector-Vector}

\begin{tabular}{ccccl}
\hline$p_{1}(\mu)$ & $p_{2}(\nu)$ & $p_{3}(\rho)$ & $p_{4}(\sigma)$ & \\
\hline$W^{+}$ & $W^{-}$ & $A$ & $A$ & $\left(2 \delta Y+2 \delta Z_{W}^{1 / 2}+2 \delta Z_{A A}^{1 / 2}\right)\langle W W A A\rangle$ \\
& & & & $+2 \delta Z_{Z A}^{1 / 2}\langle W W A Z\rangle$ \\
\hline$W^{+}$ & $W^{-}$ & $A$ & $Z$ & $\left(2 \delta Y+\delta G_{1}+2 \delta Z_{W}^{1 / 2}+\delta Z_{A A}^{1 / 2}+\delta Z_{Z Z}^{1 / 2}\right)\langle W W A Z\rangle$ \\
& & & & $+\delta Z_{A Z}^{1 / 2}\langle W W A A\rangle+\delta Z_{Z A}^{1 / 2}\langle W W Z Z\rangle$ \\
\hline$W^{+}$ & $W^{-}$ & $Z$ & $Z$ & $\left(2 \delta Y+2 \delta G_{1}+2 \delta Z_{W}^{1 / 2}+2 \delta Z_{Z Z}^{1 / 2}\right)\langle W W Z Z\rangle$ \\
& & & & $+2 \delta Z_{A Z}^{1 / 2}\langle W W A Z\rangle$ \\
\hline$W^{+}$ & $W^{-}$ & $W^{-}$ & $W^{+}$ & $\left(2 \delta Y+2 \delta G_{2}+4 \delta Z_{W}^{1 / 2}\right)\langle W W W W\rangle$ \\
\hline
\end{tabular}




\section{F.6 Vector-Vector-Scalar-Scalar}

\begin{tabular}{|c|c|c|c|c|}
\hline$p_{1}(\mu)$ & $p_{2}(\nu)$ & $p_{3}$ & $p_{4}$ & \\
\hline$A$ & $W^{ \pm}$ & $H$ & $\chi^{\mp}$ & $\begin{array}{l}\left(2 \delta Y+\delta G_{2}+\delta Z_{A A}^{1 / 2}+\delta Z_{W}^{1 / 2}+\delta Z_{H}^{1 / 2}+\delta Z_{\chi}^{1 / 2}\right)\langle A W H \chi\rangle \\
+\delta Z_{Z A}^{1 / 2}\langle Z W H \chi\rangle\end{array}$ \\
\hline$A$ & $W^{ \pm}$ & $\chi_{3}$ & $\chi^{\mp}$ & $\begin{array}{l}\left(2 \delta Y+\delta G_{2}+\delta Z_{A A}^{1 / 2}+\delta Z_{W}^{1 / 2}+\delta Z_{\chi_{3}}^{1 / 2}+\delta Z_{\chi}^{1 / 2}\right)\left\langle A W \chi_{3} \chi\right. \\
+\delta Z_{Z A}^{1 / 2}\left\langle Z W \chi_{3} \chi\right\rangle\end{array}$ \\
\hline$Z$ & $W^{ \pm}$ & $H$ & $\chi^{\mp}$ & $\begin{array}{l}\left(2 \delta Y+\delta G_{3}+\delta Z_{Z Z}^{1 / 2}+\delta Z_{W}^{1 / 2}+\delta Z_{H}^{1 / 2}+\delta Z_{\chi}^{1 / 2}\right)\langle Z W H \chi\rangle \\
+\delta Z_{A Z}^{1 / 2}\langle A W H \chi\rangle\end{array}$ \\
\hline$Z$ & $W^{ \pm}$ & $\chi_{3}$ & $\chi^{\mp}$ & $\begin{array}{l}\left(2 \delta Y+\delta G_{3}+\delta Z_{Z Z}^{1 / 2}+\delta Z_{W}^{1 / 2}+\delta Z_{\chi_{3}}^{1 / 2}+\delta Z_{\chi}^{1 / 2}\right)\left\langle Z W \chi_{3} \chi\right. \\
+\delta Z_{A Z}^{1 / 2}\left\langle A W \chi_{3} \chi\right\rangle\end{array}$ \\
\hline$A$ & $A$ & $\chi^{+}$ & $\chi^{-}$ & $\left(2 \delta Y+2 \delta Z_{A A}^{1 / 2}+2 \delta Z_{\chi}^{1 / 2}\right)\langle A A \chi \chi\rangle+2 \delta Z_{Z A}^{1 / 2}\langle Z A \chi \chi\rangle$ \\
\hline$Z$ & $A$ & $\chi^{+}$ & $\chi^{-}$ & $\begin{array}{l}\left(2 \delta Y+\delta G_{4}+\delta Z_{Z Z}^{1 / 2}+\delta Z_{A A}^{1 / 2}+2 \delta Z_{\chi}^{1 / 2}\right)\langle Z A \chi \chi\rangle \\
+\delta Z_{Z A}^{1 / 2}\langle Z Z \chi \chi\rangle+\delta Z_{A Z}^{1 / 2}\langle A A \chi \chi\rangle\end{array}$ \\
\hline$Z$ & $Z$ & $\chi^{+}$ & $\chi^{-}$ & $\begin{array}{l}\left(2 \delta Y+2 \delta G_{4}+2 \delta Z_{Z Z}^{1 / 2}+2 \delta Z_{\chi}^{1 / 2}\right)\langle Z Z \chi \chi\rangle \\
+2 \delta Z_{A Z}^{1 / 2}\langle Z A \chi \chi\rangle\end{array}$ \\
\hline$W^{+}$ & $W^{-}$ & $H$ & $H$ & $\left(2 \delta Y+2 \delta G_{2}+2 \delta Z_{W}^{1 / 2}+2 \delta Z_{H}^{1 / 2}\right)\langle W W H H\rangle$ \\
\hline$W^{+}$ & $W^{-}$ & $\chi_{3}$ & $\chi_{3}$ & $\left(2 \delta Y+2 \delta G_{2}+2 \delta Z_{W}^{1 / 2}+2 \delta Z_{\chi_{3}}^{1 / 2}\right)\left\langle W W \chi_{3} \chi_{3}\right\rangle$ \\
\hline$W^{+}$ & $W^{-}$ & $\chi^{-}$ & $\chi^{+}$ & $\left(2 \delta Y+2 \delta G_{2}+2 \delta Z_{W}^{1 / 2}+2 \delta Z_{\chi}^{1 / 2}\right)\langle W W \chi \chi\rangle$ \\
\hline$Z$ & $Z$ & $H$ & $H$ & $\left(2 \delta Y+2 \delta G_{2}+2 \delta G_{3}+2 \delta Z_{Z Z}^{1 / 2}+2 \delta Z_{H}^{1 / 2}\right)\langle Z Z H H\rangle$ \\
\hline$Z$ & $Z$ & $\chi_{3}$ & $\chi_{3}$ & $\left(2 \delta Y+2 \delta G_{2}+2 \delta G_{3}+2 \delta Z_{Z Z}^{1 / 2}+2 \delta Z_{\chi_{3}}^{1 / 2}\right)\left\langle Z Z \chi_{3} \chi_{3}\right\rangle$ \\
\hline$Z$ & $A$ & $H$ & $H$ & $\delta Z_{Z A}^{1 / 2}\langle Z Z H H\rangle \quad$ (new) \\
\hline$Z$ & $A$ & $\chi_{3}$ & $\chi_{3}$ & $\delta Z_{Z A}^{1 / 2}\left\langle Z Z \chi_{3} \chi_{3}\right\rangle$ \\
\hline
\end{tabular}




\section{F.7 Scalar-Scalar-Scalar-Scalar}

\begin{tabular}{|c|c|c|c|c|}
\hline$p_{1}$ & & $p_{3}$ & $p_{4}$ & \\
\hline$H$ & $H$ & $H$ & $H$ & $\begin{array}{l}{\left[\left(2 \delta Y+2 \delta G_{2}-2 \delta G_{W}+\delta G_{H}+4 \delta Z_{H}^{1 / 2}\right)\right.} \\
\left.-\delta T \frac{e}{s_{W} M_{W} M_{H}^{2}}\right]\langle H H H H\rangle\end{array}$ \\
\hline$\chi_{3}$ & $\chi_{3}$ & $\chi_{3}$ & $\chi_{3}$ & $\begin{array}{l}{\left[\left(2 \delta Y+2 \delta G_{2}-2 \delta G_{W}+\delta G_{H}+4 \delta Z_{\chi_{3}}^{1 / 2}\right)\right.} \\
\left.-\delta T \frac{e}{s_{W} M_{W} M_{H}^{2}}\right]\left\langle\chi_{3} \chi_{3} \chi_{3} \chi_{3}\right\rangle\end{array}$ \\
\hline$\chi^{ \pm}$ & $\chi^{\mp}$ & $\chi^{\mp}$ & $\chi^{ \pm}$ & $\begin{array}{l}{\left[\left(2 \delta Y+2 \delta G_{2}-2 \delta G_{W}+\delta G_{H}+4 \delta Z_{\chi}^{1 / 2}\right)\right.} \\
\left.-\delta T \frac{e}{s_{W} M_{W} M_{H}^{2}}\right]\langle\chi \chi \chi \chi\rangle\end{array}$ \\
\hline$H$ & $H$ & $\chi_{3}$ & $\chi_{3}$ & $\begin{array}{l}{\left[\left(2 \delta Y+2 \delta G_{2}-2 \delta G_{W}+\delta G_{H}+2 \delta Z_{H}^{1 / 2}+2 \delta Z_{\chi^{3}}^{1 / 2}\right)\right.} \\
\left.-\delta T \frac{e}{s_{W} M_{W} M_{H}^{2}}\right]\left\langle H H \chi_{3} \chi_{3}\right\rangle\end{array}$ \\
\hline$H$ & $H$ & $\chi^{+}$ & $\chi^{-}$ & $\begin{array}{l}{\left[\left(2 \delta Y+2 \delta G_{2}-2 \delta G_{W}+\delta G_{H}+2 \delta Z_{H}^{1 / 2}+2 \delta Z_{\chi}^{1 / 2}\right)\right.} \\
\left.-\delta T \frac{e}{s_{W} M_{W} M_{H}^{2}}\right]\langle H H \chi \chi\rangle\end{array}$ \\
\hline$\chi^{+}$ & $\chi^{-}$ & $\chi_{3}$ & $\chi_{3}$ & $\begin{array}{l}{\left[\left(2 \delta Y+2 \delta G_{2}-2 \delta G_{W}+\delta G_{H}+2 \delta Z_{\chi}^{1 / 2}+2 \delta Z_{\chi_{3}}^{1 / 2}\right)\right.} \\
\left.-\delta T \frac{e}{s_{W} M_{W} M_{H}^{2}}\right]\left\langle\chi \chi \chi_{3} \chi_{3}\right\rangle\end{array}$ \\
\hline
\end{tabular}

\section{F.8 Fermion-Fermion-Vector}

We define $L, R=\left(1 \mp \gamma_{5}\right) / 2$.

\begin{tabular}{|c|c|c|c|}
\hline$p_{1}$ & $p_{2}$ & $p_{3}(\mu)$ & \\
\hline $\bar{f}$ & $f$ & $A$ & $\begin{array}{l}\left(\delta Y+\delta Z_{A A}^{1 / 2}+2 \delta Z_{f L}^{1 / 2}\right) e Q_{f} \gamma^{\mu} L \\
+\left(\delta Y+\delta Z_{A A}^{1 / 2}+2 \delta Z_{f R}^{1 / 2}\right) e Q_{f} \gamma^{\mu} R \\
+\delta Z_{Z A}^{1 / 2} \frac{e}{2 s_{W} c_{W}}\left(2 I_{3} \gamma^{\mu} L-2 s_{W}^{2} Q_{f} \gamma^{\mu}(L+R)\right)\end{array}$ \\
\hline $\bar{f}$ & $f$ & $\begin{array}{c}Z \\
+(\delta Y-\delta \\
+(\delta Y-\delta\end{array}$ & $\begin{array}{l}\quad\left(\delta Y+\delta G_{2}+\delta G_{3}+\delta Z_{Z Z}^{1 / 2}+2 \delta Z_{f L}^{1 / 2}\right) \frac{e}{2 s_{W} c_{W}} 2 I_{3} \gamma^{\mu} L \\
\left.G_{2}+\delta G_{3}+\delta Z_{Z Z}^{1 / 2}+2 \delta Z_{f L}^{1 / 2}\right) \frac{e}{2 s_{W} c_{W}}\left(-2 s_{W}^{2} Q_{f} \gamma^{\mu} L\right) \\
\left.G_{2}+\delta G_{3}+\delta Z_{Z Z}^{1 / 2}+2 \delta Z_{f R}^{1 / 2}\right) \frac{e}{2 s_{W} c_{W}}\left(-2 s_{W}^{2} Q_{f} \gamma^{\mu} R\right) \\
+\delta Z_{A Z}^{1 / 2} e Q_{f} \gamma^{\mu}(L+R)\end{array}$ \\
\hline $\bar{U} / \bar{D}$ & $D / U$ & $W^{+} / W^{-}$ & $\left(\delta Y+\delta G_{2}+\delta Z_{(U / D) L}^{1 / 2}+\delta Z_{(D / U) L}^{1 / 2}+\delta Z_{W}^{1 / 2}\right) \frac{e}{\sqrt{2} s_{W}} \gamma^{\mu} L$ \\
\hline
\end{tabular}




\section{F.9 Fermion-Fermion-Scalar}

$L, R=\left(1 \mp \gamma_{5}\right) / 2$

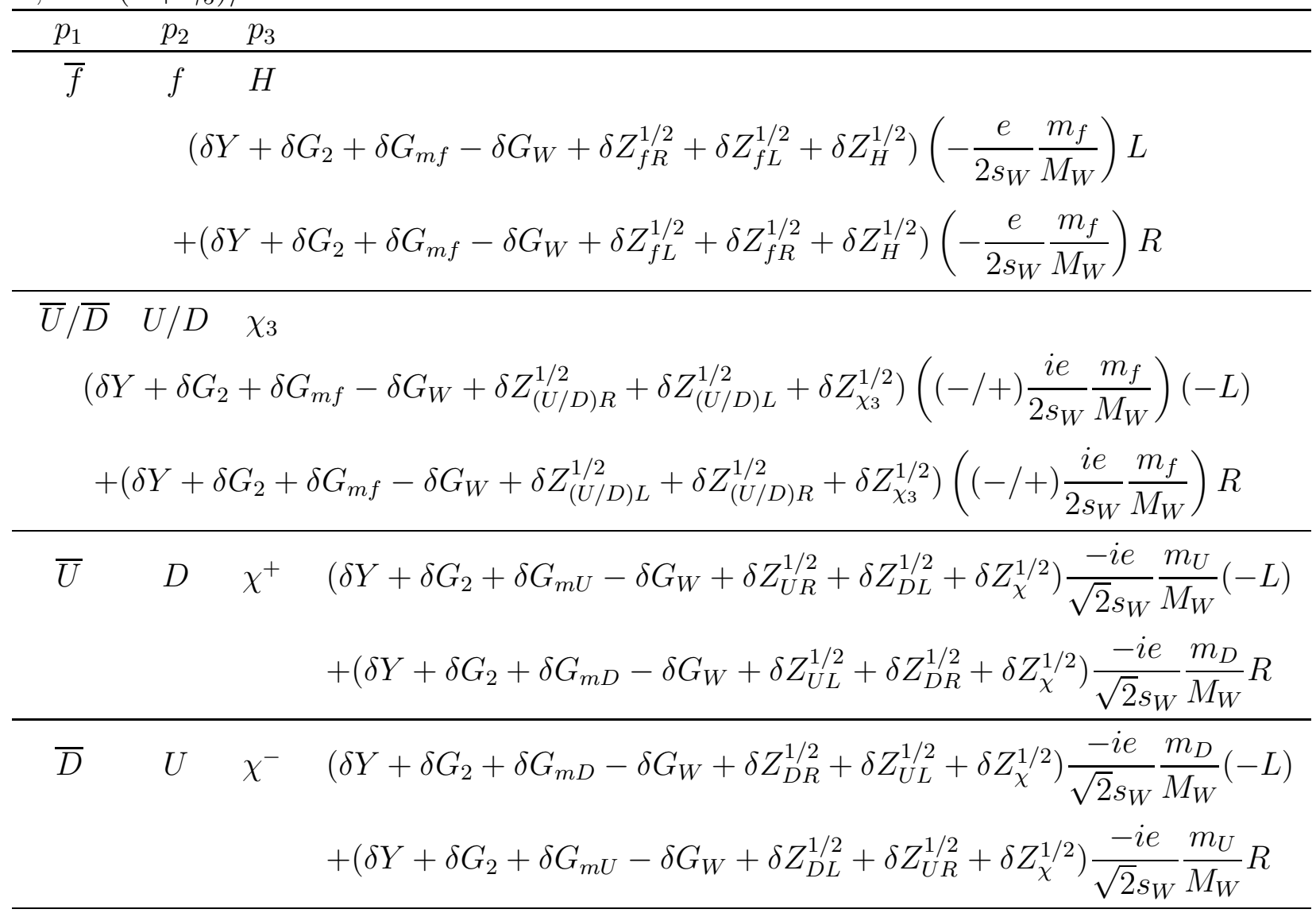

\section{G Properties of two-point functions}

As mentioned earlier, loop integrals are calculated using dimensional regularisation. In the following $l$ will be the loop momentum. Since one-point and two-point functions (tadpoles and self-energies) are essential in the derivation of the counterterms, we list here the properties of these functions.

For the one-point function, which corresponds for example to the diagram shown in Fig_18.(a), we have the well known result:

$$
\int \frac{d^{n} \ell}{i(2 \pi)^{n}} \frac{1}{\ell^{2}-m_{A}^{2}}=\frac{1}{16 \pi^{2}} m_{A}^{2}\left(C_{U V}-\log m_{A}^{2}+1\right)
$$

where $C_{U V}$ is defined in Eq. 4.9.

A typical two-point function refers to a diagram as shown in Fig 18 . 
Figure 18: Diagrams for the one-point (a) and two-point functions (b).

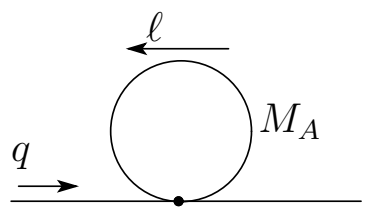

(a)

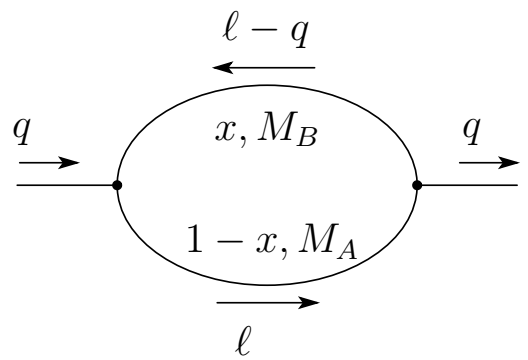

(b)

This leads to the calculation of

$$
\begin{aligned}
& \int \frac{d^{n} \ell}{i(2 \pi)^{n}} \frac{N(l)}{\left(\ell^{2}-M_{A}^{2}\right)\left((\ell-q)^{2}-M_{B}^{2}\right)} \\
& =\int \frac{d^{n} \ell}{i(2 \pi)^{n}} \int_{0}^{1} d x \frac{N}{\left[(1-x)\left(\ell^{2}-M_{A}^{2}\right)+x\left((\ell-q)^{2}-M_{B}^{2}\right)\right]^{2}} .
\end{aligned}
$$

where $N(l)$ depends, in general, on the momenta $l, q$ and the masses. Defining $D_{2}$ as

$$
D_{2}=(1-x) M_{A}^{2}+x M_{B}^{2}-x(1-x) s, \quad\left(s=q^{2}\right)
$$

we usually need to compute

$$
\begin{gathered}
\int \frac{d^{n} \ell}{i(2 \pi)^{n}} \frac{1}{\left(\ell^{2}-D_{2}\right)^{2}}=\frac{1}{16 \pi^{2}}\left(C_{U V}-\log D_{2}\right) \\
\int \frac{d^{n} \ell}{i(2 \pi)^{n}} \frac{\ell^{2}}{\left(\ell^{2}-D_{2}\right)^{2}}=\frac{1}{16 \pi^{2}} 2 D_{2}\left(C_{U V}+\frac{1}{2}-\log D_{2}\right) \\
\int \frac{d^{n} \ell}{i(2 \pi)^{n}} \frac{\ell^{\mu} \ell^{\nu}}{\left(\ell^{2}-D_{2}\right)^{2}}=\frac{1}{16 \pi^{2}} \frac{D_{2}}{2}\left(C_{U V}+1-\log D_{2}\right) g^{\mu \nu}
\end{gathered}
$$

Then the integral over the parameter $x$ gives

$$
\begin{gathered}
\int_{0}^{1} d x D_{2}=\frac{1}{2}\left(M_{A}^{2}+M_{B}^{2}\right)-\frac{1}{6} s \\
F_{n}(A, B)=\int_{0}^{1} d x x^{n} \log D_{2}=\int_{0}^{1} d x x^{n} \log \left[(1-x) M_{A}^{2}+x M_{B}^{2}-x(1-x) s\right]
\end{gathered}
$$


We do not show the explicit form of $F_{n}$ in terms of elementary functions (the result is well known). We only encounter $n=0,1,2$.

The notation $F(A, B)=F_{1}(A, B)-F_{2}(A, B)=F(B, A)$ is sometimes used.

$$
\begin{aligned}
\tilde{F}(A, B)= & \int_{0}^{1} d x D_{2} \log D_{2} \\
& =M_{A}^{2}\left(F_{0}(A, B)-F_{1}(A, B)\right)+M_{B}^{2} F_{1}(A, B)-s F(A, B)
\end{aligned}
$$

We have several relations for the $F_{n}$ functions as shown below. All $F_{n}$ can be reduced into $F_{0}$.

Exchange of $\mathrm{A}$ and $\mathrm{B}$

$$
\begin{aligned}
& F_{0}(B, A)=F_{0}(A, B) \\
& F_{1}(B, A)=F_{0}(A, B)-F_{1}(A, B) \\
& F_{2}(B, A)=F_{0}(A, B)-2 F_{1}(A, B)+F_{2}(A, B)
\end{aligned}
$$

$\underline{\text { Reduction into } F_{0}, A \neq B}$

$$
\begin{gathered}
F_{1}(A, B)=\frac{1}{2}\left(1+\frac{M_{A}^{2}-M_{B}^{2}}{s}\right) F_{0}(A, B)+\frac{1}{2 s}\left(M_{B}^{2} \log M_{B}^{2}-M_{A}^{2} \log M_{A}^{2}-M_{B}^{2}+M_{A}^{2}\right) \\
F_{2}(A, B)=\frac{2}{3}\left(1+\frac{M_{A}^{2}-M_{B}^{2}}{s}\right) F_{1}(A, B)-\frac{M_{A}^{2}}{3 s} F_{0}(A, B) \\
+\frac{1}{3 s}\left(M_{B}^{2} \log M_{B}^{2}+\frac{1}{2}\left(M_{A}^{2}-M_{B}^{2}\right)\right)-\frac{1}{18}
\end{gathered}
$$

$\underline{\text { Reduction into } F_{0}, A=B}$

$$
\begin{gathered}
F_{1}(A, A)=\frac{1}{2} F_{0}(A, A) \\
F_{2}(A, A)=\frac{1}{3}\left(1-\frac{M_{A}^{2}}{s}\right) F_{0}(A, A)+\frac{M_{A}^{2}}{3 s} \log M_{A}^{2}-\frac{1}{18}
\end{gathered}
$$

$\underline{G \text { functions (Derivative of } F \text { ) }}$

$$
G_{n}(A, B)=\frac{d}{d s} F_{n}(A, B)=\int_{0}^{1} d x \frac{-x^{n} \cdot x(1-x)}{D_{2}}
$$


$F$ and $G$ at special energy

$$
\begin{aligned}
& F_{n}(A, B ; C)=\left.F_{n}(A, B)\right|_{s=M_{C}^{2}} \\
& F_{n}(A, B ; 0)=\left.F_{n}(A, B)\right|_{s=0} \\
& G_{n}(A, B ; C)=\left.G_{n}(A, B)\right|_{s=M_{C}^{2}} \\
& G_{n}(A, B ; 0)=\left.G_{n}(A, B)\right|_{s=0}
\end{aligned}
$$

$\underline{F_{0} \text { for } s=0}$

$$
F_{0}(A, B, 0)= \begin{cases}\log M_{A}^{2} & (A=B) \\ \frac{M_{B}^{2} \log M_{B}^{2}-M_{A}^{2} \log M_{A}^{2}}{M_{B}^{2}-M_{A}^{2}}-1 & (A \neq B)\end{cases}
$$

\section{H Results for the one-loop corrections to the propa- gators}

We here give the details on the calculation of the loop corrections to the various propagators and mixings. For the vector bosons we present both the transverse and longitudinal part as defined in Section 6.3. We also show the various contributions by classifying them according to the diagrams of Fig. 18. Therefore for each propagator we show a table containing the two types of diagrams. The fermion contributions are summed over all fermion species in the case of the neutral sector and over all doublets in the charged sector. In both cases summing over colour for quarks is implied. Before presenting the results for the two-point function, we first start by presenting the one-loop contribution to the tadpole. Although we will require this contribution to vanish against the tadpole counterterm we give its full expression for completeness. Also, the latter is needed for the Ward identities.

\section{H.1 The tadpole}

The tadpole contribution $T^{\text {loop }}$ only receives contributions from diagrams of the type shown in Fig. 18-(a) where $A=W, Z, \chi, \chi_{3}, H, c, c^{Z}, f$. The result is as follows: 


$$
\begin{aligned}
T^{\text {loop }}= & \frac{e}{16 \pi^{2} s_{W} M_{W}}\left[M_{W}^{2}\left(\left(C_{U V}-\log M_{W}^{2}+1\right)\left(3 M_{W}^{2}+\frac{1}{2} M_{H}^{2}\right)-2 M_{W}^{2}\right)\right. \\
& +M_{Z}^{2}\left(\left(C_{U V}-\log M_{Z}^{2}+1\right)\left(\frac{3}{2} M_{Z}^{2}+\frac{1}{4} M_{H}^{2}\right)-M_{Z}^{2}\right) \\
& +\frac{3}{4} M_{H}^{4}\left(C_{U V}-\log M_{H}^{2}+1\right) \\
& \left.-\sum_{f} 2 m_{f}^{4}\left(C_{U V}-\log m_{f}^{2}+1\right)\right]
\end{aligned}
$$

It is important to note that all dependence on the non-linear gauge parameters (namely $\tilde{\varepsilon}$ and $\tilde{\delta}$ ) vanishes among all diagrams and is therefore the same as in the usual linear gauge. This can be considered as a check on the calculation, since the tadpole $T$ can be considered as a basic parameter of the theory.

From this expression we immediately derive the tadpole counterterm:

$$
\delta T=-T^{l o o p} .
$$

\section{H.2 $A-A$}

For all the two-point functions we will list, as done in the table below, the contributing diagrams where (a) corresponds to the type shown in Fig. 18-(a) and (b) to Fig. 18. (b)

\begin{tabular}{l|l}
\hline (b) & $(A, B)=(W, W),(W, \chi),(\chi, W),(\chi, \chi),\left(c^{+}, c^{+}\right),\left(c^{-}, c^{-}\right),(f, f)$ \\
\hline (a) & $A=W, \chi, c^{+}, c^{-}$ \\
\hline
\end{tabular}

$$
\begin{aligned}
\Pi_{T}^{A A}\left(q^{2}\right) & =\frac{\alpha}{4 \pi} q^{2}\left[7 C_{U V}-5 F_{0}(W, W)-12 F(W, W)+\frac{2}{3}-4(1-\tilde{\alpha})\left(C_{U V}-F_{0}(W, W)\right)\right. \\
& \left.-8 \sum_{f} Q_{f}^{2}\left(\frac{1}{6} C_{U V}-F(f, f)\right)\right] .
\end{aligned}
$$

Note that independently of the gauge parameter $\Pi_{T}^{A A}(0)=0$. This is just a remnant of the QED gauge invariance which is explicit at one-loop. This also gives $\Pi_{L}^{A A}\left(q^{2}\right)=0$ which we explicitly verify. More generally we will also check explicitly that in both the linear and non-linear gauges $\Pi_{T}(0)=\Pi_{L}(0)$ for all vector-vector transitions. This is another check on the calculation and encodes the property that there is no spurious pole in the propagators essential for the Goldstone mechanism. 
H.3 $Z-A$

$$
\begin{aligned}
& \begin{array}{l|l}
\hline \text { (b) } & (A, B)=(W, W),(W, \chi),(\chi, W),(\chi, \chi),\left(c^{+}, c^{+}\right),\left(c^{-}, c^{-}\right),(f, f) \\
\hline \text { (a) } & A=W, \chi, c^{+}, c^{-} \\
\hline
\end{array} \\
& \Pi_{T}^{Z A}=\frac{\alpha}{4 \pi} \frac{c_{W}}{s_{W}}\left\{q ^ { 2 } \left[C_{U V}\left(7+\frac{1}{6 c_{W}^{2}}\right)-4\left(3-\frac{1}{2 c_{W}^{2}}\right) F(W, W)+\frac{2}{3}-\left(5+\frac{1}{2 c_{W}^{2}}\right) F_{0}(W, W)\right.\right. \\
& -2(1-\tilde{\beta})\left(C_{U V}-F_{0}(W, W)\right) \\
& \left.-\frac{2}{c_{W}^{2}} \sum_{f}\left|Q_{f}\right|\left(1-4\left|Q_{f}\right| s_{W}^{2}\right)\left(\frac{1}{6} C_{U V}-F(f, f)\right)\right] \\
& \left.-2(1-\tilde{\alpha})\left(q^{2}-M_{Z}^{2}\right)\left(C_{U V}-F_{0}(W, W)\right)\right\}, \\
& \Pi_{L}^{Z A}=\frac{\alpha}{2 \pi} \frac{c_{W}}{s_{W}}(1-\tilde{\alpha}) M_{Z}^{2}\left(C_{U V}-F_{0}(W, W)\right)
\end{aligned}
$$

Note that we do get as a check that $\Pi_{T}^{Z A}(0)=\Pi_{L}^{Z A}(0)$. Moreover for $\tilde{\alpha}=1$ this condition is even stronger since we get $\Pi_{T}^{Z A}(0)=\Pi_{L}^{Z A}=0$. This is due to the fact that for this particular choice of the parameter, the gauge-fixing in the charged sector which contributes here (note that fermions do not contribute to $\Pi_{L}^{Z A}$ ), there is an additional $U(1)_{\text {QED }}$ gauge invariance. This choice is therefore very useful. As we will see this is also responsible for the vanishing of the induced $A-\chi_{3}$ transition, see section H.8.1. It is also important to remark that at the $Z$-pole the $\tilde{\alpha}$ dependence vanishes. This is also responsible for the fact that the counterterms needed for the mass definitions do not depend on the gauge fixing.

\section{H.4 Z $Z$ Z}

\begin{tabular}{l|l}
\hline (b) & $(A, B)=(W, W),(W, \chi),(\chi, W),(\chi, \chi),(H, \chi),(H, Z),\left(c^{+}, c^{+}\right),\left(c^{-}, c^{-}\right),(f, f)$ \\
\hline (a) & $A=W, H, \chi_{3}, \chi, c^{+}, c^{-}$ \\
\hline
\end{tabular}

$$
\begin{gathered}
\Pi_{T}^{Z Z}=\frac{\alpha}{4 \pi s_{W}^{2} c_{W}^{2}}\left(T_{b}^{Z Z}+T_{f}^{Z Z}+(1-\tilde{\beta})\left(q^{2}-M_{Z}^{2}\right) \Delta T^{Z Z}\right), \\
T_{b}^{Z Z}=C_{U V}\left[q^{2}\left(7 c_{W}^{4}-\frac{1-2 c_{W}^{2}}{6}\right)-2 M_{W}^{2}-M_{Z}^{2}\right]+\frac{2}{3} q^{2} c_{W}^{4}-\frac{q^{2}}{12} \\
-8 q^{2} c_{W}^{4} F_{0}(W, W)+q^{2}\left(F_{0}(W, W)-4 F(W, W)\right)\left(3 c_{W}^{4}+\frac{1-4 c_{W}^{2}}{4}\right)
\end{gathered}
$$




$$
\begin{aligned}
& +2 M_{W}^{2} F_{0}(W, W)+\frac{q^{2}}{2} F(H, Z)-\frac{M_{H}^{2}}{2} F_{0}(H, Z)-\frac{M_{Z}^{2}-M_{H}^{2}}{2} F_{1}(H, Z) \\
& +M_{Z}^{2} F_{0}(H, Z)+\frac{1}{4}\left(M_{H}^{2} \log M_{H}^{2}+M_{Z}^{2} \log M_{Z}^{2}\right), \\
T_{f}^{Z Z} & =-\frac{1}{2} \sum_{f}\left[\left(\left(1-4\left|Q_{f}\right| s_{W}^{2}\right)^{2}+1\right)\left(\frac{1}{6} C_{U V}-F(f, f)\right) q^{2}-m_{f}^{2}\left(C_{U V}-F_{0}(f, f)\right)\right],
\end{aligned}
$$

$$
\Delta T^{Z Z}=-4 c_{W}^{4}\left(C_{U V}-F_{0}(W, W)\right)
$$

We have,

$$
\begin{gathered}
\Pi_{\text {NonLinear }}^{T}\left(M_{Z}^{2}\right)=\Pi_{\text {Linear }}^{T}\left(M_{Z}^{2}\right) . \\
\Pi_{L}^{Z Z}=\frac{\alpha}{16 \pi s_{W}^{2} c_{W}^{2}}\left\{q ^ { 2 } \left[C_{U V} \tilde{\epsilon}^{2}-\frac{1}{3}+2 F(H, Z)-(1-\tilde{\epsilon})^{2} F_{0}(H, Z)-4 F_{2}(H, Z)\right.\right. \\
\left.\quad+4(1-\tilde{\epsilon}) F_{1}(H, Z)\right] \\
+4 M_{Z}^{2}\left(C_{U V}-F_{0}(H, Z)\right)-2 M_{H}^{2} F_{0}(H, Z)-2\left(M_{Z}^{2}-M_{H}^{2}\right) F_{1}(H, Z) \\
+\left(M_{H}^{2} \log M_{H}^{2}+M_{Z}^{2} \log M_{Z}^{2}\right)-8 M_{W}^{2}\left(C_{U V}-F_{0}(W, W)\right)\left(1-2 c_{W}^{2}(1-\tilde{\beta})\right) \\
\left.+2 \sum_{f} m_{f}^{2}\left(C_{U V}-F_{0}(f, f)\right)\right\} .
\end{gathered}
$$

It is easy to see that, for any choice of the gauge parameters, $\Pi_{L}^{Z Z}(0)=\Pi_{T}^{Z Z}(0)$ which is a check on the calculation. Also note that the $\tilde{\epsilon}$ dependence is proportional to $q^{2}$.

\section{H.5 $W-W$}

$$
\begin{aligned}
& \begin{array}{l|l}
\hline \text { (b) } & (A, B)=(Z, W),(Z, \chi),(A, W),(A, \chi),(H, \chi),(H, W),\left(\chi_{3}, \chi\right), \\
& \left(c^{Z}, c^{+}\right),\left(c^{Z}, c^{-}\right),\left(c^{A}, c^{+}\right),\left(c^{A}, c^{-}\right),\left(f, f^{\prime}\right) \\
\hline \text { (a) } & A=A, Z, W, H, \chi_{3}, \chi, c^{+}, c^{-} \\
\hline
\end{array} \\
& \Pi_{T}^{W W}=\frac{\alpha}{4 \pi s_{W}^{2}}\left(T_{b}^{W W}+T_{f}^{W W}+\left(q^{2}-M_{W}^{2}\right) \Delta T_{\tilde{\alpha}, \tilde{\beta}}^{W W}\right) \\
& T_{b}^{W W}=C_{U V}\left(\frac{19}{6} q^{2}+2 M_{W}^{2}-M_{Z}^{2}\right)-\frac{q^{2}}{6}
\end{aligned}
$$




$$
\begin{aligned}
& +4 s_{W}^{2}\left[q^{2}\left(F(A, W)-F_{0}(A, W)\right)-M_{W}^{2} F_{1}(A, W)\right] \\
& +c_{W}^{2}\left[4 q^{2}\left(F(Z, W)-F_{0}(Z, W)\right)-4\left(M_{W}^{2}-M_{Z}^{2}\right) F_{1}(Z, W)\right. \\
& \left.+\left(-7 M_{Z}^{2}+\frac{M_{Z}^{2}}{c_{W}^{2}}\right) F_{0}(Z, W)\right]+\frac{q^{2}}{2}(F(H, W)+F(Z, W))+M_{W}^{2} F_{0}(H, W) \\
& -\frac{1}{2}\left[M_{H}^{2} F_{0}(H, W)+M_{Z}^{2} F_{0}(Z, W)+\left(M_{W}^{2}-M_{H}^{2}\right) F_{1}(H, W)\right. \\
& \left.+\left(M_{W}^{2}-M_{Z}^{2}\right) F_{1}(Z, W)\right] \\
& +\frac{5 M_{W}^{2}}{2} \log M_{W}^{2}+\frac{M_{Z}^{2}}{4} \log M_{Z}^{2}+\frac{M_{H}^{2}}{4} \log M_{H}^{2}+2 M_{W}^{2} \log M_{Z}^{2}, \\
T_{f}^{W W}= & -\frac{1}{2} \sum_{\text {doublet }}\left\{4\left(\frac{1}{6} C_{U V}-F\left(f, f^{\prime}\right)\right) q^{2}-\left(m_{f}^{2}+m_{f}^{\prime 2}\right) C_{U V}+2 m_{f}^{2} F_{1}\left(f^{\prime}, f\right)\right. \\
& \left.+2 m_{f}^{\prime 2} F_{1}\left(f, f^{\prime}\right)\right\} \\
\Delta T_{\tilde{\alpha}, \tilde{\beta}}^{W W}= & -2\left(s_{W}^{2} \tilde{\alpha}\left(C_{U V}-F_{0}(A, W)\right)+c_{W}^{2} \tilde{\beta}\left(C_{U V}-F_{0}(Z, W)\right)\right) .
\end{aligned}
$$

Here also we check that ,

$$
\begin{aligned}
& \Pi_{\text {NonLinear }}^{T}\left(M_{W}^{2}\right)=\Pi_{\text {Linear }}^{T}\left(M_{W}^{2}\right) \\
& \Pi_{L}^{W W}= \frac{\alpha}{4 \pi s_{W}^{2}}\left\{C_{U V}\left(2 M_{W}^{2}-M_{Z}^{2}\right)-\frac{5}{6} q^{2}\right. \\
&+ 2 s_{W}^{2}\left[q^{2}\left(6 F(A, W)-F_{0}(A, W)\right)-2 M_{W}^{2} F_{1}(A, W)\right] \\
&+ c_{W}^{2}\left[2 q^{2}\left(6 F(Z, W)-F_{0}(Z, W)\right)-4\left(M_{W}^{2}-M_{Z}^{2}\right) F_{1}(Z, W)\right. \\
&\left.+\left(\frac{M_{Z}^{2}}{c_{W}^{2}}-7 M_{Z}^{2}\right) F_{0}(Z, W)\right] \\
&+ \frac{1}{2}\left[q^{2}\left(3 F(H, W)-\frac{F_{0}(H, W)}{2}\right)+2 M_{W}^{2} F_{0}(H, W)-M_{H}^{2} F_{0}(H, W)\right. \\
&\left.\quad-\left(M_{W}^{2}-M_{H}^{2}\right) F_{1}(H, W)\right] \\
&+ \frac{1}{2}\left[q^{2}\left(3 F(Z, W)-\frac{F_{0}(Z, W)}{2}\right)-M_{Z}^{2} F_{0}(Z, W)-\left(M_{W}^{2}-M_{Z}^{2}\right) F_{1}(Z, W)\right] \\
&+ \frac{5 M_{W}^{2}}{2} \log M_{W}^{2}+\frac{M_{Z}^{2}}{4} \log M_{Z}^{2}+\frac{M_{H}^{2}}{4} \log M_{H}^{2}+2 M_{W}^{2} \log M_{Z}^{2} \\
&+ 2 s_{W}^{2} \tilde{\alpha}\left[3 q^{2}\left(F_{0}(A, W)-2 F_{1}(A, W)\right)+M_{W}^{2}\left(C_{U V}-F_{0}(A, W)\right)\right] \\
&+ 2 c_{W}^{2} \tilde{\beta}\left[3 q^{2}\left(F_{0}(Z, W)-2 F_{1}(Z, W)\right)+M_{W}^{2}\left(C_{U V}-F_{0}(Z, W)\right)\right]
\end{aligned}
$$




$$
\begin{aligned}
& +s_{W}^{2} \tilde{\alpha}^{2} q^{2}\left(5 C_{U V}-6 F_{0}(A, W)+2 F_{1}(A, W)-2\right) \\
& +c_{W}^{2} \tilde{\beta}^{2} q^{2}\left(5 C_{U V}-6 F_{0}(Z, W)+2 F_{1}(Z, W)-2\right) \\
& +\frac{\tilde{\delta}}{2} q^{2}\left(F_{0}(H, W)-2 F_{1}(H, W)\right)+\frac{\tilde{\kappa}}{2} q^{2}\left(F_{0}(Z, W)-2 F_{1}(Z, W)\right) \\
& +\frac{\tilde{\delta}^{2}}{4} q^{2}\left(C_{U V}-F_{0}(H, W)\right)+\frac{\tilde{\kappa}^{2}}{4} q^{2}\left(C_{U V}-F_{0}(Z, W)\right) \\
& \left.+\frac{1}{2} \sum_{\text {doublet }}\left\{\left(m_{f}^{2}+m_{f}^{\prime 2}\right) C_{U V}-2 m_{f}^{2} F_{1}\left(f^{\prime}, f\right)-2 m_{f}^{\prime 2} F_{1}\left(f, f^{\prime}\right)\right\}\right\} .
\end{aligned}
$$

We again have that, for any choice of the gauge parameters, $\Pi_{L}^{W W}(0)=\Pi_{T}^{W W}(0)$ which is a check on the calculation. Also note that the $\tilde{\delta}, \tilde{\kappa}$ dependence is proportional to $q^{2}$, as is any dependence quadratic in $\tilde{\alpha}, \tilde{\beta}$. All these dependencies will be present in the propagators/mixings of the Goldstones.

Note that for both the $W W$ and $Z Z$ transition the tadpole contribution is not included as it will be canceled against that of the tadpole counterterms. Moreover note that such contribution do not depend on the gauge parameter. On the other hand the inclusion of the tadpole one-loop correction is needed for the Ward identities relating the bosonic two-point functions.

\section{H.6 $H-H$}

\begin{tabular}{l|l}
\hline (b) & $(A, B)=(W, W),(W, \chi),(\chi, W),(\chi, \chi),\left(Z, \chi_{3}\right),\left(\chi_{3}, \chi_{3}\right),(Z, Z),(H, H)$, \\
& $\left(c^{+}, c^{+}\right),\left(c^{-}, c^{-}\right),\left(c^{Z}, c^{Z}\right),(f, f)$
\end{tabular}

Here we explicitly add the tadpole contribution.

$$
\Pi^{H}\left(q^{2}\right)+\frac{3 \delta T}{v}=\frac{\alpha}{4 \pi s_{W}^{2}}\left(\Pi_{b}^{H}+\Pi_{f}^{H}+\left(q^{2}-M_{H}^{2}\right) \Pi_{\tilde{\delta}, \tilde{\epsilon}}^{H}\right)
$$

with

$$
\begin{aligned}
\Pi_{b}^{H}= & C_{U V}\left[-\left(q^{2}+\frac{M_{H}^{2}}{2}\right)\left(1+\frac{1}{2 c_{W}^{2}}\right)+\frac{3 M_{H}^{4}}{4 M_{W}^{2}}\right]-\frac{9 M_{H}^{4}}{8 M_{W}^{2}} F_{0}(H, H) \\
& -F_{0}(W, W)\left(-q^{2}+3 M_{W}^{2}+\frac{M_{H}^{4}}{4 M_{W}^{2}}\right)-\frac{F_{0}(Z, Z)}{2 c_{W}^{2}}\left(-q^{2}+3 M_{Z}^{2}+\frac{M_{H}^{4}}{4 M_{Z}^{2}}\right) \\
& -\left(\frac{M_{H}^{2}}{2}+3 M_{W}^{2}\right)\left(1-\log M_{W}^{2}\right)-\frac{1}{2 c_{W}^{2}}\left(\frac{M_{H}^{2}}{2}+3 M_{Z}^{2}\right)\left(1-\log M_{Z}^{2}\right)
\end{aligned}
$$




$$
\begin{gathered}
-\frac{3 M_{H}^{4}}{4 M_{W}^{2}}\left(1-\log M_{H}^{2}\right), \\
\Pi_{f}^{H}=\sum_{f} \frac{m_{f}^{2}}{M_{W}^{2}}\left\{\frac{q^{2}}{2}\left(C_{U V}-F_{0}(f, f)\right)+2 m_{f}^{2}\left(1-\log m_{f}^{2}+F_{0}(f, f)\right)\right\}, \\
\prod_{\tilde{\delta}, \tilde{\epsilon}}^{H}=\left(-C_{U V}+F_{0}(W, W)\right) \tilde{\delta}+\left(-C_{U V}+F_{0}(Z, Z)\right) \frac{\tilde{\epsilon}}{2 c_{W}^{2}} .
\end{gathered}
$$

Again at $q^{2}=M_{H}^{2}$ the self-energy is independent of the gauge parameter, which means that the shift in the Higgs mass will also not depend on the gauge parameters.

\section{H.7 $f-f$}

At one-loop the result is the same as in the linear gauge, but we give here the full result that includes mass effects as well as the contribution of the Goldstones. We have neglected all fermion mixing. The $K_{j}^{f}$ have been introduced in section 6.2 and correspond to the different Lorentz structures of the fermion propagator. Since we are neglecting mixing and assuming $\mathcal{C P}$-invariance $K_{5}^{f}=0$ holds. We have also found it convenient to express each of these Lorentz coefficients in a basis that corresponds to the various contributions to the self energy (photon exchange, W-exchange, etc...).

$$
\begin{aligned}
& \begin{array}{l|l}
\hline \text { (b) } & (A, B)=(f, A),(f, Z),\left(f^{\prime}, W\right),(f, H),\left(f, \chi_{3}\right),\left(f^{\prime}, \chi\right) \\
\hline \text { (a) } & \text { None } \\
\hline
\end{array} \\
& K_{j}^{f}(s)=\frac{\alpha}{4 \pi}\left[Q_{f}^{2} K_{j}^{A}+\frac{1}{c_{W}^{2}} Q_{f}^{2} s_{W}^{2} K_{j}^{Z(1)}-\frac{1}{2 c_{W}^{2}}\left|Q_{f}\right| K_{j}^{Z(2)}+\frac{1}{8 s_{W}^{2} c_{W}^{2}} K_{j}^{Z(3)}\right. \\
& \left.+\frac{1}{4 s_{W}^{2}} K_{j}^{W}+\frac{1}{4 s_{W}^{2} c_{W}^{2}} \frac{m_{f}^{2}}{M_{Z}^{2}} K_{j}^{S}\right] \quad(j=1, \gamma, 5 \gamma) \\
& K_{1}^{A}=m_{f}\left[-4 C_{U V}+2+4 F_{0}(f, A)\right] \\
& K_{\gamma}^{A}=C_{U V}-1-2 F_{1}(f, A), \\
& K_{5 \gamma}^{A}=0 \\
& K_{1}^{Z(1)}=m_{f}\left[-4 C_{U V}+2+4 F_{0}(f, Z)\right], \quad K_{1}^{Z(2)}=K_{1}^{Z(1)}, \quad K_{1}^{Z(3)}=0, \\
& K_{\gamma}^{Z(1)}=C_{U V}-1-2 F_{1}(f, Z), \quad K_{\gamma}^{Z(2)}=K_{\gamma}^{Z(1)}, \quad K_{\gamma}^{Z(3)}=K_{\gamma}^{Z(1)} \\
& K_{5 \gamma}^{Z(1)}=0, \quad K_{5 \gamma}^{Z(2)}=-K_{\gamma}^{Z(1)}, \quad K_{5 \gamma}^{Z(3)}=-K_{\gamma}^{Z(1)},
\end{aligned}
$$




$$
\begin{gathered}
K_{1}^{W}=0 \\
K_{\gamma}^{W}=C_{U V}-1-2 F_{1}\left(f^{\prime}, W\right), \\
K_{5 \gamma}^{W}=-K_{\gamma}^{W}, \\
K_{1}^{S}=m_{f}\left[-F_{0}(f, H)+F_{0}(f, Z)-2 \frac{m_{f}^{\prime 2}}{m_{f}^{2}}\left(C_{U V}-F_{0}\left(f^{\prime}, W\right)\right)\right], \\
K_{\gamma}^{S}=C_{U V}-F_{1}(f, H)-F_{1}(f, Z)+\frac{1}{2}\left(1+\frac{m_{f}^{\prime 2}}{m_{f}^{2}}\right)\left(C_{U V}-2 F_{1}\left(f^{\prime}, W\right)\right), \\
K_{5 \gamma}^{S}=+\frac{1}{2}\left(1-\frac{m_{f}^{\prime 2}}{m_{f}^{2}}\right)\left(C_{U V}-2 F_{1}\left(f^{\prime}, W\right)\right) .
\end{gathered}
$$

\section{H.8 The Goldstone sector}

We do not need to be explicit about the renormalisation of this sector in order to arrive at finite S-matrix elements. Nonetheless we list all the vector-Goldstone mixings and Goldstone propagators.

H.8.1 $A-\chi_{3}$

\begin{tabular}{l|l}
\hline (b) & $(A, B)=(W, \chi),(\chi, W),\left(c^{+}, c^{+}\right),\left(c^{-}, c^{-}\right),(f, f)$ \\
\hline (a) & None \\
\hline
\end{tabular}

There is no fermionic contribution.

$$
\Pi^{A \chi_{3}}\left(q^{2}\right)=\frac{\alpha M_{W}}{2 \pi s_{W}}(1-\tilde{\alpha})\left(C_{U V}-F_{0}(W, W)\right) .
$$

As expected this does vanish for $\tilde{\alpha}=1$ and is a remnant of the $U(1)_{\text {QED }}$ gauge invariance for this value of the parameter.

\section{H.8.2 $Z-\chi_{3}$}

\begin{tabular}{l|l}
\hline (b) & $(A, B)=(W, \chi),(\chi, W),\left(H, \chi_{3}\right),(H, Z),\left(c^{+}, c^{+}\right),\left(c^{-}, c^{-}\right),(f, f)$ \\
\hline (a) & None \\
\hline
\end{tabular}




$$
\begin{aligned}
\Pi_{Z \chi_{3}} & =\frac{\alpha M_{Z}}{8 \pi s_{W}^{2} c_{W}^{2}}\left\{c_{W}^{2}\left(-3+4 c_{W}^{2}(1-\tilde{\beta})-\tilde{\kappa}\right)\left(C_{U V}-F_{0}(W, W)\right)\right. \\
& +\frac{M_{H}^{2}}{2 M_{Z}^{2}}\left[F_{0}(H, Z)-2 F_{1}(H, Z)\right]-\left[\frac{3 C_{U V}}{2}-F_{1}(H, Z)-F_{0}(H, Z)\right] \\
& +\tilde{\epsilon}\left[\frac{C_{U V}}{2}-F_{1}(H, Z)+\frac{M_{H}^{2}}{2 M_{Z}^{2}}\left(C_{U V}-F_{0}(H, Z)\right]\right. \\
& +\tilde{\epsilon}^{2}\left(C_{U V}-F_{0}(H, Z)\right) \\
& \left.+\sum_{f} \frac{m_{f}^{2}}{M_{Z}^{2}}\left(C_{U V}-F_{0}(f, f)\right)\right\} .
\end{aligned}
$$

H.8.3 $W-\chi$

\begin{tabular}{l|l}
\hline (b) & $(A, B)=(H, W),(H, \chi),(Z, \chi),(Z, W),(A, \chi),(A, W)$, \\
& $\left(c^{A}, c\right),\left(c^{Z}, c^{+}\right),\left(c^{Z}, c^{-}\right),\left(f, f^{\prime}\right)$ \\
\hline (a) & None \\
\hline
\end{tabular}

$$
\begin{aligned}
\Pi_{W \chi_{+}} & =\frac{\alpha M_{W}}{16 \pi s_{W}^{2}}\left\{C_{U V}\left(2-\frac{3}{c_{W}^{2}}\right)\right. \\
& +C_{U V}\left(\frac{M_{H}^{2}}{M_{W}^{2}} \tilde{\delta}+\tilde{\kappa}+\tilde{\delta}+2 \tilde{\delta}^{2}+s_{W}^{2}\left(18 \tilde{\alpha}^{2}-12 \tilde{\alpha}\right)+4 \tilde{\beta}\left(4-3 c_{W}^{2}\right)+18 c_{W}^{2} \tilde{\beta}^{2}\right) \\
& +4 s_{W}^{2}\left(4 F_{1}(A, W)-F_{0}(A, W)\right) \\
& +4 s_{W}^{2} \tilde{\alpha}\left(-6 F_{1}(A, W)+6 F_{0}(A, W)-5 \tilde{\alpha} F_{0}(A, W)+\tilde{\alpha} F_{1}(A, W)\right) \\
& +F_{1}(Z, W)\left(s_{W}^{2}\left(-16-\frac{2}{c_{W}^{2}}\right)-2 \tilde{\kappa}-24 c_{W}^{2} \tilde{\beta}+4 c_{W}^{2} \tilde{\beta}^{2}\right) \\
& +F_{0}(Z, W)\left(2-4 c_{W}^{2}+4 \frac{s_{W}^{2}}{c_{W}^{2}}-16 \tilde{\beta}+24 c_{W}^{2} \tilde{\beta}-20 c_{W}^{2} \tilde{\beta}^{2}\right) \\
& +\frac{M_{H}^{2}}{M_{W}^{2}}\left(2 F_{1}(W, H)-F_{0}(W, H)(1+\tilde{\delta})\right)+2\left(2 F_{0}(W, H)-F_{1}(W, H)\right) \\
& +2 \tilde{\delta}\left(F_{1}(W, H)-F_{0}(W, H)(1+\tilde{\delta})\right) \\
& +8 \tilde{\alpha} s_{W}^{2}(1-\tilde{\alpha})-8 \tilde{\beta}\left(1-c_{W}^{2}(1-\tilde{\beta})\right) \\
& \left.+4 \sum_{d o u b l e t} \frac{1}{M_{W}^{2}}\left[\frac{m_{f}^{2}+m_{f}^{\prime 2}}{2} C_{U V}-\left(m_{f}^{\prime 2} F_{0}\left(f^{\prime}, f\right)+m_{f}^{\prime 2} F_{1}\left(f, f^{\prime}\right)\right)\right]\right\} .
\end{aligned}
$$


H.8.4 $\chi_{3}-\chi_{3}$

\begin{tabular}{l|l}
\hline (b) & $(A, B)=(W, \chi),(\chi, W),(H, Z),\left(H, \chi_{3}\right),\left(c^{+}, c^{+}\right),\left(c^{-}, c^{-}\right),(f, f)$ \\
\hline (a) & $A=W, Z, \chi_{3}, \chi, H, c^{+}, c^{-}, c^{Z}$ \\
\hline
\end{tabular}

The second line of $\Pi^{\chi_{3}}$ shows the fermionic contributions. Since the tadpole contribution appears with $\Pi^{\chi_{3}}$, we present the formula for the sum. We note that $C^{\chi_{3}}$, the coefficient for the divergent part, is proportional to $s$ in the linear gauge.

$$
\begin{gathered}
\Pi^{\chi_{3}}\left(q^{2}\right)+\frac{\delta T}{v}=\frac{\alpha}{16 \pi s_{W}^{2}}\left[C^{\chi_{3}} C_{U V}+d_{W W}^{\chi_{3}} F_{0}(W, W)+d_{H Z}^{\chi_{3}} F_{0}(H, Z)+d_{0}^{\chi_{3}}\right. \\
\left.+2 q^{2} \sum_{f} \frac{m_{f}^{2}}{M_{W}^{2}}\left(C_{U V}-F_{0}(f, f)\right)\right] \\
C^{\chi_{3}}=-\left(\frac{2}{c_{W}^{2}}+4\right) q^{2}+\tilde{\varepsilon} \frac{2}{c_{W}^{2}}\left(M_{H}^{2}+q^{2}\right)+\tilde{\varepsilon}^{2} \frac{3}{c_{W}^{2}} M_{Z}^{2}-4 \tilde{\kappa} q^{2} \\
d_{W W}^{\chi_{3}}=4 q^{2}(1+\tilde{\kappa}) \\
d_{H Z}^{\chi_{3}}=\frac{1}{c_{W}^{2}}\left(2 M_{H}^{2}-M_{Z}^{2}+2 q^{2}-\frac{\left(M_{H}^{2}\right)^{2}}{M_{Z}^{2}}-2 \tilde{\varepsilon}\left(M_{H}^{2}+q^{2}\right)-3 \tilde{\varepsilon}^{2} M_{Z}^{2}\right) \\
d_{0}^{\chi_{3}}=\frac{1}{c_{W}^{2}}\left[\left(\frac{\left(M_{H}^{2}\right)^{2}}{M_{Z}^{2}}-M_{H}^{2}\right) \log M_{H}^{2}+\left(M_{Z}^{2}-M_{H}^{2}\right) \log M_{Z}^{2}-\frac{\left(M_{H}^{2}\right)^{2}}{M_{Z}^{2}}+2 M_{H}^{2}-M_{Z}^{2}\right]
\end{gathered}
$$

\section{H.8.5 $\chi-\chi$}

\begin{tabular}{l|l}
\hline (b) & $(A, B)=(H, W),(H, \chi),\left(\chi_{3}, W\right),(Z, \chi),(Z, W),(A, \chi),(A, W),\left(c^{Z}, c^{+}\right),\left(c^{Z}, c^{-}\right),\left(f, f^{\prime}\right)$ \\
\hline (a) & $A=A, Z, W, H, \chi_{3}, \chi, c^{+}, c^{-}$ \\
\hline
\end{tabular}

The second line of $\Pi^{\chi}$ shows the fermionic contribution. Since the tadpole contribution appears with $\Pi^{\chi}$, we present the formula for the sum. We note that $C^{\chi}$, the coefficient for the divergent part, is proportional to $s$ in the linear gauge. 


$$
\begin{aligned}
& \Pi^{\chi}\left(q^{2}\right)+\frac{\delta T}{v}=\frac{\alpha}{16 \pi s_{W}^{2}}\left[C^{\chi} C_{U V}+d_{Z W}^{\chi} F_{0}(Z, W)+d_{H W}^{\chi} F_{0}(H, W)+d_{A W}^{\chi} F_{0}(A, W)+d_{0}^{\chi}\right. \\
& \left.+2 q^{2} \sum_{f} \frac{m_{f}^{2}}{M_{W}^{2}}\left(C_{U V}-2 F_{1}\left(f^{\prime}, f\right)\right)\right] \\
& C^{\chi}=-\left(\frac{2}{c_{W}^{2}}+4\right) q^{2}-32 \tilde{\alpha} s_{W}^{2} M_{W}^{2}+32 \tilde{\beta} s_{W}^{2} M_{W}^{2}+16 \tilde{\alpha}^{2} s_{W}^{2} M_{W}^{2}+16 \tilde{\beta}^{2} c_{W}^{2} M_{W}^{2} \\
& +2 \tilde{\delta}\left(q^{2}+M_{H}^{2}\right)+3 \tilde{\delta}^{2} M_{W}^{2}+2 \tilde{\kappa} q^{2}-\tilde{\kappa}^{2} M_{W}^{2}, \\
& d_{Z W}^{\chi}=\left(2-8 s_{W}^{2}+\frac{2}{c_{W}^{2}}\right) q^{2}-16 c_{W}^{2} M_{W}^{2}-\frac{6}{c_{W}^{2}} M_{W}^{2}-\frac{1}{c_{W}^{2}} M_{Z}^{2}-8 s_{W}^{2} M_{W}^{2}+23 M_{W}^{2} \\
& -32 \tilde{\beta} s_{W}^{2} M_{W}^{2}-16 \tilde{\beta}^{2} c_{W}^{2} M_{W}^{2}-2 \tilde{\kappa} q^{2}+\tilde{\kappa}^{2} M_{W}^{2} \text {, } \\
& d_{H W}^{\chi}=2 q^{2}-M_{W}^{2}+2 M_{H}^{2}-\frac{\left(M_{H}^{2}\right)^{2}}{M_{W}^{2}}-2 \tilde{\delta}\left(s+M_{H}^{2}\right)-3 \tilde{\delta}^{2} M_{W}^{2} \\
& d_{A W}^{\chi}=8 s_{W}^{2}\left(s-M_{W}^{2}\right)+32 \tilde{\alpha} s_{W}^{2} M_{W}^{2}-16 \tilde{\alpha}^{2} s_{W}^{2} M_{W}^{2}, \\
& d_{0}^{\chi}=\log M_{W}^{2}\left(-M_{H}^{2}+2 M_{W}^{2}-M_{Z}^{2}\right)+\log M_{H}^{2}\left(\frac{\left(M_{H}^{2}\right)^{2}}{M_{W}^{2}}-M_{H}^{2}\right) \\
& +\log M_{Z}^{2}\left(\frac{M_{Z}^{2}}{c_{W}^{2}}+8 s_{W}^{2} M_{Z}^{2}-M_{Z}^{2}\right)-\frac{\left(M_{H}^{2}\right)^{2}}{M_{W}^{2}}+2 M_{H}^{2}+6 M_{W}^{2}-\frac{M_{Z}^{2}}{c_{W}^{2}}-6 M_{Z}^{2} \\
& +16 \tilde{\alpha} s_{W}^{2} M_{W}^{2}-16 \tilde{\beta} s_{W}^{2} M_{W}^{2}-8 \tilde{\alpha}^{2} s_{W}^{2} M_{W}^{2}-8 \tilde{\beta}^{2} c_{W}^{2} M_{W}^{2} .
\end{aligned}
$$

\section{Direct determination of the charge counterterm}

A direct derivation of the charge counterterm $\delta Y$ necessitates the calculation of the vertex $e^{+} e^{-} A$ with on-shell electrons and in the Thomson limit where the photon momentum $q \rightarrow 0, \Gamma_{e}^{\mu}(0)$. In this limit one can relate the vertex to the electron self-energy as in 
Figure 19: Electron self energy and $e^{+} e^{-} A$ vertex.
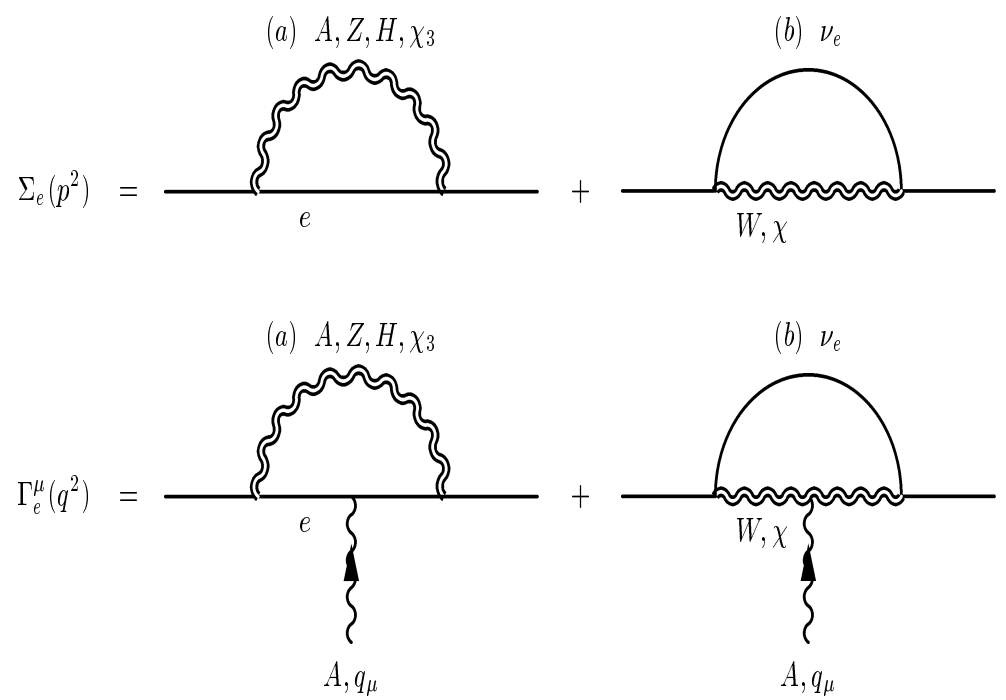

depicted Fig [19 ( $p$ is the electron momentum and $l$ is the integration momentum). Due to the identities

$$
\frac{\partial}{\partial p_{\mu}}\left(\frac{-1}{\not p+\ell-m}\right)=\frac{-1}{\not p+\ell-m} \gamma^{\mu} \frac{-1}{\not p+\ell-m},
$$

and

$$
\frac{\partial}{\partial p_{\mu}}\left(\frac{1}{(p+\ell)^{2}-M^{2}}\right)=\frac{-2(p+\ell)^{\mu}}{\left((p+\ell)^{2}-M^{2}\right)^{2}},
$$

The major part of $\Gamma_{e}^{\mu}(0)$ can be calculated by the corresponding fermion self energy. Using the notation in Fig 19.

$$
\begin{aligned}
& \text { (a) } A, Z, H, \chi_{3} \quad \Gamma_{e}^{\mu}(0)=\left.(-e) \frac{\partial}{\partial p_{\mu}} \Sigma\left(p^{2}\right)\right|_{\not p=m}, \\
& \text { (b) } W, \chi \quad \Gamma_{e}^{\mu}(0)=\left.(-e) \frac{\partial}{\partial p_{\mu}} \Sigma\left(p^{2}\right)\right|_{\not p=m}+G_{e}^{\mu} .
\end{aligned}
$$

It is only $G_{e}^{\mu}$ which is gauge-parameter dependent, in fact it only depends on $\tilde{\alpha}$ and vanishes for $\tilde{\alpha}=1$.

$$
G_{e}^{\mu}=-\frac{\alpha}{4 \pi} \frac{e}{s_{W}^{2}}(1-\tilde{\alpha})\left(C_{U V}-\log M_{W}^{2}\right) \gamma^{\mu} L
$$


We note that for this particular value of the gauge parameter there is a residual $U(1)_{\text {QED }}$ symmetry and therefore it is no wonder that the naive Ward identity is verified in this case in Eq. I.4.

The gauge-parameter independent part of $\Gamma_{\mu}(0)$ is derived from the (on-shell) electron self-energy. We obtain

$$
\begin{aligned}
\left.\frac{\partial}{\partial p_{\mu}} \Sigma\left(p^{2}\right)\right|_{\not p=m} & =\left(2 m_{e} K_{1}^{\prime}\left(m_{e}^{2}\right)+2 m_{e}^{2} K_{\gamma}^{\prime}\left(m_{e}^{2}\right)+K_{\gamma}\left(m_{e}^{2}\right)\right) \gamma^{\mu}+K_{5 \gamma}\left(m_{e}^{2}\right) \gamma^{\mu} \gamma_{5} \\
& =-2 \delta Z_{e L}^{1 / 2} \gamma^{\mu} L-2 \delta Z_{e R}^{1 / 2} \gamma^{\mu} R .
\end{aligned}
$$

Here Eq 6.20 is used.

The counterterm for the $e^{+} e^{-} A$ vertex, $\Gamma_{e}^{\mu}(0)$, is defined in Sec F.8. Adding this to the loop calculation we get

$$
\begin{aligned}
\tilde{\Gamma}_{e}^{\mu}(0) & =\Gamma_{e}^{\mu}(0)+\hat{\Gamma}_{e}^{\mu}(0) \\
& =\left(-e \gamma^{\mu}\right)\left(\delta Y+\delta Z_{A A}^{1 / 2}-\frac{s_{W}}{c_{W}} \delta Z_{Z A}^{1 / 2}\right)+\left[-\frac{e}{2 s_{W} c_{W}} \delta Z_{Z A}^{1 / 2} \gamma^{\mu} L+G_{e}^{\mu}\right] .
\end{aligned}
$$

The second term (within square brackets) vanishes identically. Imposing the renormalisation condition $(\mathrm{Eq} 6.21) \tilde{\Gamma}_{e}^{\mu}(0)=0$,

$$
\delta Y=-\delta Z_{A A}^{1 / 2}+\frac{s_{W}}{c_{W}} \delta Z_{Z A}^{1 / 2}
$$

and we find the linear gauge result

$$
\delta Y=\frac{\alpha}{4 \pi}\left\{-\frac{7}{2}\left(C_{U V}-\log M_{W}^{2}\right)-\frac{1}{3}+\frac{2}{3} \sum_{f} Q_{f}^{2}\left(C_{U V}-\log m_{f}^{2}\right)\right\} .
$$

\section{J Graph theory and optimization in the generation of Feynman diagrams}

We shall first introduce some technical terms. In the following let us call a vertex or an external particle a node. Similarly, let an edge be a connection between two nodes, which may be a propagator or a connection between a vertex and an external particle. Thus an edge is expressed by a pair of two nodes (which are connected by the edge). The graph generation process is to construct edges in all possible ways. Although vertices of the same kind are not distinguished from each other, they will be distinguished in a program, usually through a sequence of numbered labels. Since a Feynman graph is a 
topological object, it is independent of the way one assigns the sequence of numbers to nodes. Therefore two graphs are topologically the same when there is a permutation in the sequential numbers in a graph which produces another graph. Some permutations acting on a graph will keep the graph unchanged. These permutations form a symmetry group of the graph whose number of elements is needed to calculate the symmetry factor required for the calculation of the Feynman amplitudes.

Let us consider three-loop vacuum graphs in the $\phi^{3}$ model for example. There are two one-particle irreducible Feynman graphs as shown in Fig 20. A permutation of any two nodes in the graph of Fig[20a produces the same graph. However in graph Fig[20], the exchange of nodes 1 and 2 gives different numbering of the topologically equivalent graph, while the simultaneous exchange of nodes $1 \leftrightarrow 2$ and $3 \leftrightarrow 4$ results in the same graph with the same numbering. When a permutation of nodes in a graph produces a different representation of the graph, one must remove either the original graph or the new one obtained by the permutation. When the permutation keeps a graph invariant, it is found to be an element of the symmetry group of the graph. This symmetry structure

Figure 20: Three-loop vacuum graphs in $\phi^{3}$ model
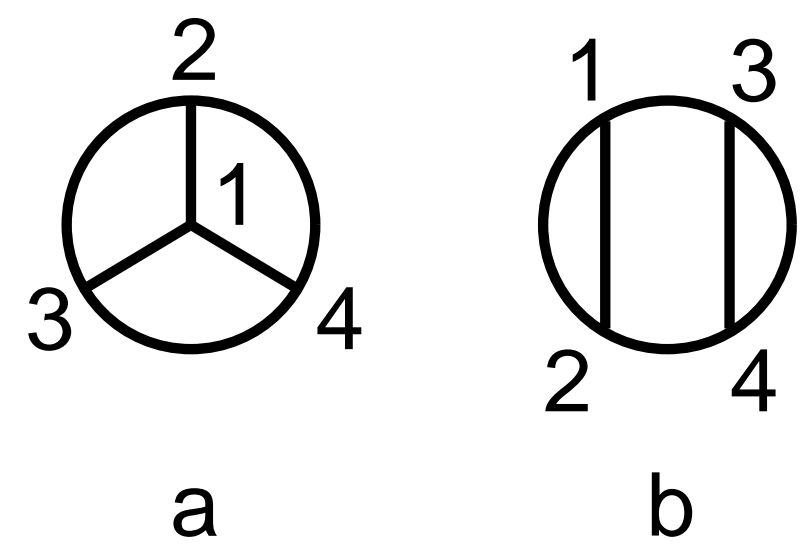

of a graph originates from the fact that a vertex is symmetric under the exchange of the three legs. When the vertex does not have such a symmetry as in the case of QED, it is possible to avoid the duplication of graphs at all orders of the coupling constant 18. Generation of tree-level graphs is also easy since a node is distinguished from others by the set of momenta entering the node. The most difficult cases are vacuum graphs of the $\phi^{3}$ or $\phi^{4}$ model at the multi-loop level, since all nodes in a graph are equivalent. Fortunately, the calculation of decay or scattering processes involves external particles that make the distinction simpler. This also makes the code for the generation of graphs simpler and faster.

The first idea to avoid duplication is to keep already generated graphs in the main memory 
or the hard disks in order to compare them with the newly generated ones [27]. However as the number of graphs grows as the factorial of the number of nodes, it is impossible to keep all the graphs in the main memory. One is then forced to move all the generated graphs onto the hard disk. This very much slows down the code. A more efficient method has been developed by graph theorists to avoid generating duplicated graphs [67]. It checks a generated graph with the one obtained through permutation and therefore requires memory space just for two graphs. The outline of this method is the following:

1. Let $P$ be the set of all permutations among nodes of a graph $G$ and $\sigma(G)$ be a graph obtained by applying a permutation $\sigma \in P$ to a graph $G$. The set of all topologically equivalent graphs of $G$ is $\{\sigma(G) \mid \sigma \in P\}$.

2. We introduce the connection matrix $M$ of a graph $G$, whose matrix element $M_{i j}$ is 1 when nodes $i$ and $j$ are connected by an edge and is 0 otherwise. We apply the permutation $\sigma$ to this matrix. When the permuted matrix of a graph is identical to the matrix of another graph, these two graphs are topologically the same.

3. By lexicographical comparison of matrix elements among two connection matrices we can introduce an ordering relation, $\succ$, among graphs. Among all topologically identical graphs, we keep only the "larger" graph in this comparison. This means that we discard graph $G$ when there exists a permutation $\sigma$ such that $\sigma(G) \succ G$.

4. The resulting algorithm is as follows:

(a) When a graph $G$ is generated, generate all possible permutations $\sigma$ of the nodes.

(b) The connection matrix of $G$ is compared with one of $\sigma(G)$.

(c) If $\sigma(G) \succ G$, the graph $G$ is discarded. Otherwise another permutation is tried in the same way.

(d) When $G$ is found to satisfy $G \succeq \sigma(G)$ for all possible permutations, $G$ is kept.

(e) At the same time, the number $N_{s}$ of such permutations $\sigma$ is counted that satisfy $G=\sigma(G)$.

5. The symmetry factor of $G$ is given by $1 /\left(N_{s} \times N_{e}\right)$, where $N_{e}$ is the number of permutations among edges that keep $G$ invariant.

This method was first applied to the generation of Feynman graphs in the code QGRAF[21]. Even with this method, it is necessary to compare two graphs. Unfortunately, no efficient algorithm which avoids the factorial growth from all permutations and that terminates within a number of steps which is a polynomial function of the size of the graph is known $[68]^{15}$. The size is of course determined by the number of nodes. In

\footnotetext{
${ }^{15}$ Such efficient algorithms in graph theory are known as polynomial-time algorithms.
} 
order to speed up the code, one has to decrease the number of generated graphs and the number of permutations to be tested in a consistent way ${ }^{16}$. A method for acceleration by a systematic classification of graphs proposed in [69] is used in the GRACE system.

Programs can be checked by two counting methods [21]. First the number of generated graphs for the $\phi^{3}$ or $\phi^{4}$ model is compared with what is predicted by a graph theoretical method. Second the sum of the number of graphs weighted by the symmetry factor is compared with the value calculated in zero-dimensional field theory.

\footnotetext{
${ }^{16}$ QGRAPH seems to include some acceleration method but this is not described in [21.
} 


\section{References}

[1] G. Bélanger, F. Boudjema, J. Fujimoto, T. Ishikawa, T. Kaneko, K. Kato and Y. Shimizu, Nucl. Phys. (Proc. Suppl.) 116 (2003) 353; hep-ph/0211268.

[2] G. Bélanger, F. Boudjema, J. Fujimoto, T. Ishikawa, T. Kaneko, K. Kato and Y. Shimizu, Phys. Lett. B559 (2003) 252; hep-ph/0212261.

[3] A. Denner, S. Dittmaier, M. Roth and M.M. Weber, Phys. Lett. B560 (2003) 196; hep-ph/0301189 and Nucl. Phys. B660 (2003)289; hep-ph/0302198.

[4] For a review of some of these techniques and achievements, see Z. Bern, Nucl. Phys. (Proc.Suppl). 117 (2003); hep-ph/0212406.

[5] E. Witten, Commun. Math. Phys. , 252 (2004) 189; hep-th/0312171.

[6] A. Brandhuber, B. Spence and G. Travaglini, Nucl. Phys. B706 (2005) 150; hepth/0407214.

[7] V.V. Khoze, hep-th/0408233.

[8] I. Bena, Z. Bern, D.A. Kosower and R. Roiban, Phys. Rev. D71 (2005) 106010; hep-th/0410054.

[9] M. Veltman, "SCHOONSCHIP, A CDC 6600 programme for symbolic evaluation of Feynman diagrams." CERN Preprint, 1965.

H. Strubbe, Comput. Phys. Commun. 8 (1974) 1.

[10] See for example, A.C. Hearn, Commun. ACM 14 (1971) 511.

A.C. Hearn, J. Comp. Phys. 5 (1970) 280.

[11] A.C. Hearn: REDUCE User's Manual, version 3.7, Rand. Corp. 1999.

[12] J. A. M. Vermaseren:New Features of FORM; math-ph/0010025.

[13] S. Wolfram, The Mathematica Book, Wolfram Media, 5th edition, 2003. http://www. wolfram. com/.

[14] http://www.maplesoft.com/products/maple/.

[15] T. Sasaki, J. Comput. Phys. 22 (1976) 189.

[16] See for example, T. Kinoshita ed., "Quantum electrodynamics", World Scientific, Singapore, 1990.

[17] S. Kawabata, Comp. Phys. Commun. 41 (1986) 127; ibid., 88 (1995) 309. 
[18] T. Kaneko, S. Kawabata and Y. Shimizu, Comput. Phys. Commun. 43 (1987) 279.

[19] D. Perret-Gallix and W. Wojcik ed., "New Computing Techniques in Physics Research", Edition du CNRS, Paris, 1990.

[20] For a review see, R. Harlander and M. Steinhauser, Prog. Part. Nucl. Phys. 43 (1999) 167; hep-ph/9812357.

[21] P. Nogueira, J. Comput. Phys. 105 (1993) 279.

[22] L. Brücher, J. Franzkowski, and D. Kreimer, Comput. Phys. Commun. 115 (1998) 140.

[23] M. Steinhauser, Comput. Phys. Commun. 134 (2001) 335; hep-ph/0009029.

[24] S.A. Larin, F.V. Tkachov, and J.A.M. Vermaseren, Rep. No. NIKHEF-H/91-18 (Amsterdam, 1991).

[25] T. Ishikawa, T. Kaneko, K. Kato, S. Kawabata, Y. Shimizu and H. Tanaka, KEK Report 92-19, 1993, GRACE manual Ver. 1.0.

[26] A. Pukhov et al., "CompHEP user's manual, v3.3", Preprint INP MSU 98-41/542, 1998; hep-ph/9908288.

[27] R. Mertig, M. Böhm and A. Denner, Comput. Phys. Commun. 64 (1991) 345.

[28] J. Küblbeck, M. Böhm, and A. Denner, Comp. Phys. Commun. 60 (1990) 165;

H. Eck and J. Küblbeck, Guide to FeynArts 1.0 (Würzburg, 1992);

H. Eck, Guide to FeynArts 2.0 (Würzburg, 1995);

T. Hahn, Comp. Phys. Commun. 140 (2001) 418; hep-ph/0012260.

[29] R. Mertig, M. Böhm and A. Denner, Comput. Phys. Commun. 64 (1991) 345.

[30] T.Hahn and M. Perez-Victoria, Comput. Phys. Commun. 118 (1999) 153; hep$\mathrm{ph} / 9807565$.

[31] T. Hahn, Nucl. Phys. Proc. Suppl. 135 (2004) 333; hep-ph/0406288. ibid hep$\mathrm{ph} / 0506201$.

[32] T. Stelzer and W.F. Long, Phys. Commun. 81 (1994) 357; hep-ph/9401258. http://madgraph.hep.uiuc.edu/index.html.

[33] J.-X. Wang, Comput. Phys. Commun. 77 (1993) 263,

J.-X. Wang and Y. Shimizu, "New Computing Techniques in Physics Research III" K.-H. Becks and D. Perret-Gallix ed., World Scientific, Singapore, 1994, 517; http://www.ihep.ac.cn/lunwen/wjx/public_html/. 
[34] H. Tanaka et al., Nucl. Instrum. and Meth. A389 (1997) 295.

J. Fujimoto et al., Comp. Phys. Commun. 153 (2003) 106; hep-ph/0208036.

[35] F. Caravaglios and M. Moretti, Phys. Lett. B358 (1995) 332; hep-ph/9507237. F. Caravaglios and M. Moretti, Z. Physik C74 (1997) 291; hep-ph/9604316.

[36] A. Kanaki, C.G. Papadopoulos, Comput. Phys. Commun. 132 (2000) 306; hep$\mathrm{ph} / 0002082$.

[37] M. Moretti, T. Ohl and J. Reuter, hep-ph/0102195.

[38] G. 't Hooft and M. Veltman, Nucl. Phys. 44 (1972) 189.

[39] C. G. Bollini, J.J. Giambiagi and A. Gonzales Dominguez, Nuovo Cim. 31 (1964) 551.

C. G. Bollini and J.J. Giambiagi, Nuovo Cim. 12A (1972) 20.

J.F. Ashmore, Nuovo Cim. Lett. 4 (1972) 289.

G.M. Cicuta and E. Montaldi, Nuovo Cim. Lett. 4 (1972) 329.

[40] A. Denner and S. Dittmaier, Nucl. Phys. B658 (2003), 175; hep-ph/0212259.

[41] T. Hahn, LoopTools, http://www.feynarts.de/looptools/.

[42] F. Boudjema, J. Fujimoto, T. Ishikawa, T. Kaneko, K. Kato, Y. Kurihara, Y. Shimizu and Y. Yasui, Phys. Lett. B600 (2004) 65; hep-ph/0407065.

[43] G. Belanger, F. Boudjema, J. Fujimoto, T. Ishikawa, T. Kaneko, Y. Kurihara, K. Kato, and Y. Shimizu, Phys. Lett. B576 (2003) 152; hep-ph/0309010.

[44] Zhang Ren-You, Ma Wen-Gan, Chen Hui, Sun Yan-Bin, Hou Hong-Sheng, Phys. Lett. B578 (2004) 349; hep-ph/0308203.

[45] G. Bélanger, F. Boudjema, J. Fujimoto, T. Ishikawa, T. Kaneko, K. Kato, Y. Shimizu and Y. Yasui, Phys. Lett. B571 (2003) 163; hep-ph/0307029.

[46] A. Denner, S. Dittmaier, M. Roth and M.M. Weber, Phys. Lett. B575 (2003) 290; hep-ph/0307193 and Nucl. Phys. B680 (2004) 85; hep-ph/0309274.

[47] You Yu, Ma Wen-Gan, Chen Hui, Zhang Ren-You, Sun Yan-Bin and Hou HongSheng, Phys. Lett. B571 (2003) 85; hep-ph/0306036. .

[48] Chen Hui, Ma Wen-Gan, Zhang Ren-You, Zhou Pei-Jun, Hou Hong-Sheng, Nucl. Phys. B683 (2004) 196; hep-ph/0309106.

[49] F. Boudjema, J. Fujimoto, T. Ishikawa, T. Kaneko, K. Kato, Y. Kurihara, Y. Shimizu, S. Yamashita and Y. Yasui, Nucl. Instrum. and Meth. A534 (2004) 334; hep-ph/0404098. 
[50] F. Boudjema, J. Fujimoto, T. Ishikawa, T. Kaneko, K. Kato, Y. Kurihara, Y. Shimizu and Y. Yasui, hep-ph/0510184.

[51] A. Denner, S. Dittmaier, M. Roth and L.H. Wieders, Phys. Lett. B612 (2005); hep-ph/0502063. ibid Nucl. Phys. B724 (2005) 247; hep-ph/0505042.

[52] F. Boudjema, J. Fujimoto, T. Ishikawa, T. Kaneko, K. Kato, Y. Kurihara and Y. Shimizu, Nucl. Phys. Proc.Suppl. 135 (2004) 323; hep-ph/0407079.

[53] K. Fujikawa, Phys. Rev. D7 (1973) 393

M. Base and N.D. Hari Dass, Ann. Phys. 94 (1975) 349

M.B. Gavela, G. Girardi, C. Malleville and P. Sorba, Nucl. Phys. B193 (1981) 257

N.G. Deshpande and M. Nazerimonfared, Nucl. Phys. B213 (1983) 390

F. Boudjema, Phys. Lett. B187 (1987) 362.

M. Baillargeon and F. Boudjema, Phys. Lett. B272 (1991) 158, ibid B317 (1993) 371; hep-ph/9308342.

[54] F. Boudjema and E. Chopin, Z. Phys. C73 (1996) 85; hep-ph/9507396.

[55] A. Denner, G. Weiglein and S. Dittmaier, Nucl. Phys. B440 (1995) 95; hep$\mathrm{ph} / 9410338$.

[56] For some of these issues as well as a comprehensive review of radiative correction in the electroweak theory see, D. Y. Bardin and G. Passarino, The Standard Model in the making: precision study of the electroweak interactions, Clarendon Press, Oxford, U.K. (1999).

[57] H. Tanaka, Comput. Phys. Commun. 58 (1990) 153.

[58] J. Fleischer and O.V. Tarasov, Comput. Phys. Commun. 71 (1992) 193.

[59] L.V. Avdeev, J. Fleischer, M.Yu. Kalmykov, and M.N. Tentyukov, Comput. Phys. Commun. 107 (1997) 155; hep-ph/9710222.

M. Tentyukov and J. Fleischer, Comput. Phys. Commun. 132 (2000) 124; hep$\mathrm{ph} / 9904258$.

[60] A. Lorca and T. Riemann, Comput. Phys. Commun. 174 (2006) 71; hep$\mathrm{ph} / 0412047$.

[61] G. Bélanger, F. Boudjema, A. Pukhov and A. Semenov, Comput. Phys. Commun. 149 (2002) 103; hep-ph/0112278. ibid, Comput. Phys. Commun. in Press; hep$\mathrm{ph} / 0405253$.

http://wwwlapp.in2p3.fr/lapth/micromegas/. 
[62] A. Pukhov, hep-ph/0412191. See also, http://www.ifh.de/ pukhov/calchep.html.

[63] A. Semenov. LanHEP - a package for automatic generation of Feynman rules. User's manual.; hep-ph/9608488.

A. Semenov, Nucl. Inst. Meth. and Inst. A393 (1997) p. 293.

A. Semenov, Comp. Phys. Commun. 115 (1998) 124.

A. Semenov, hep-ph/0208011.

[64] T. Kaneko, Nucl. Instr. and Meth. A502 (2003) 555.

[65] C. Becchi, A. Rouet, R. Stora, Comm. Math. Phys. 42 (1975) 127.

See also, B. W. Lee, Les Houches, Session XXVIII, 1975, Methods in field theory, eds. R. Balian, J. Zinn-Justin, North Holland, Amsterdam, 1976.

[66] I.V. Tyutin, Gauge invariance in field theory and statiscal physics in operator formalism, Lebedev Institute Preprint, $\mathrm{N}^{0} 39$ (1975).

[67] See for example, R.C. Read, Lecture Notes in Mathematics 884 (1980) 77.

[68] See for example, C. M. Hoffman, Lecture Notes in Computer Science 136 SpringerVerlag, 1982.

J. Köbler, U. Schöning and J. Torán, Lecture Notes in Computer Science 577 Springer-Verlag, 1992 p.401.

[69] T. Kaneko, Comput. Phys. Commun. 92 (1995) 127; hep-th/9408107.

[70] H. Murayama, I. Watanabe, and K. Hagiwara, KEK Preport 91-11 (1992).

[71] A. Denner, H. Eck, O. Hahn and J. Küblbeck, Nucl. Phys. B387 (1992) 467.

[72] See G. P. Lepage, J. Comp. Phys. 27 (1978) 192.

[73] K. Tobimatsu and S. Kawabata, Tech. Rep. 85, Kogakuin Univ. (1998) 423.

[74] E. de Doncker, A. Gupta, J. Ball, P. Ealy and A. Genz., "Proc. of the 10th ACM International Conference on Supercomputing," (1996) 149;

http://www.cs.wmich.edu/〜parint/.

[75] W. Kilian, http://www-ttp.physik.uni-karlsruhe.de/whizard/.

[76] S. L. Adler, Phys. Rev. 177 (1969) 2426.

J. S. Bell, R. Jackiw, Nuovo Cim. 60A (1969) 47.

[77] C. Bouchiat, J. Iliopoulos, P. Meyer, Phys. Lett. 38 (1972) 519. 
[78] W. Pauli and F. Villars, Rev. Mod. Phys. 21 (1949) 433.

[79] L.H. Ryder, Quantum Field Theory, Cambridge University Press, June 6, 1996.

[80] M.E. Peskin and D.V. Schroeder, An Introduction to Quantum Field Theory, Perseus Books, Frontiers in Physics, March 1993.

[81] P. Breitenlohner, D. Maison, Commun. Math. Phys. 52 (1977) 39.

[82] G. Bonneau, Int. J. Mod. Phys. A5 (1990) 3831.

[83] F. Jegerlehner, Eur. Phys. J. C18 (2001) 673; hep-th/0005255.

[84] W. Siegel, Phys. Lett. B84 (1979) 193.

[85] W. Siegel, Phys. Lett. B94 (1980) 37.

[86] I. Jack and D.R.T. Jones, Regularisation of supersymmetric theories, in Perspectives on supersymmetry, p.149, Editor G.L. Kane; hep-ph/9707278.

[87] D.M. Capper, D.R.T. Jones and P. van Nieuwenhuizen, Nucl. Phys. B167 (1980) 479.

[88] D.Z. Freedman, K. Johnson and J.I. Latorre, Nucl. Phys. B371 (1992) 353.

P.E. Haagensen, Mod. Phys. Lett. A7 (1992) 893; hep-th/9111015.

F. del Aguila, A. Culatti, R. Muñoz Tapia and M. Pérez-Victoria, Phys. Lett. B419 (1998) 263; hep-th/9709067.

F. del Aguila, A. Culatti, R. Muñoz Tapia and M. Pérez-Victoria, Nucl. Phys. B537 (1999) 561; hep-ph/9806451.

F. del Aguila, A. Culatti, R. Muñoz Tapia and M. Pérez-Victoria, Nucl. Phys. B504 (1997) 532; hep-ph/9702342.

[89] I.J.R. Aitchison, An informal introduction to gauge field theories, Cambridge University Press, Cambridge, 1982.

[90] K. Aoki, Z. Hioki, R. Kawabe, M. Konuma and T. Muta, Suppl. Prog. Theor. Phys. 73 (1982) 1.

[91] A. Slavnov, Theor. Math. Phys. 10 (1972) 99.

J. C. Taylor, Nucl. Phys. 33 (1971) 436.

G. 't Hooft, Nucl. Phys. 35 (1971) 167.

[92] M. Böhm, H. Spiesberger and W. Hollik, Fortsch. Phys. 34 (1986) 687.

[93] A. Denner, J. Küblbeck, R. Mertig and M. Böhm, Z. Phys. C56 (1992) 261. 
[94] B.A. Kniehl, Z. Phys. C55 (1992) 605.

[95] J. Fleischer and F. Jegerlehner, Nucl. Phys. B216 (1983) 469.

[96] T. Bhattacharya and S. Willenbrock, Phys. Rev. D47 (1993) 469.

K. Melnikov, M. Spira and O. Yakovlev, Z. Phys. C64 (1994) 401; hep-ph/9405301.

B. A. Kniehl, C.P. Palisoc and A. Sirlin, Nucl. Phys. B591 (2000) 296; hepph/0007002.

[97] F. Kleefeld, AIP Conf. Proc. 660 (2003) 325; hep-ph/0211460.

[98] D. Bailin and A. Love, Introduction to Gauge Field Theory, Adam Hilger, Bristol and Boston, IOP Publishing Limiting 1986.

[99] D. Espriu, J. Manzano and P. Talavera, Phys. Rev. D66 (2002) 076002; hep$\mathrm{ph} / 0204085$.

[100] B. A. Kniehl and A. Sirlin, Phys. Lett. B530 (2002) 129; hep-ph/0110296.

[101] M. J. G. Veltman, Physica 29 (1963) 186.

[102] R. G. Stuart, Phys. Lett. B262 (1991) 113.

[103] A. Sirlin, Phys. Rev. Lett. 67 (1991) 2127, Phys. Lett. B267 (1991) 240.

[104] H. G. J. Veltman, Z. Phys. C62 (1994) 35.

[105] A. Aeppli, G. J. van Oldenborgh and D. Wyler, Nucl. Phys. B428 (1994) 126; hep-ph/9312212.

[106] Y. Kurihara, D. Perret-Gallix and Y. Shimizu, Phys. Lett. B349 (1995) 367; hep$\mathrm{ph} / 9412215$.

[107] E.N. Argyres et al., Phys. Lett. B358 (1995) 339; hep-ph/9507216.

[108] U. Baur and D. Zeppenfeld, Phys. Rev. Lett. 75 (1995) 1002; hep-ph/9503344.

[109] W. Beenakker et al., Nucl. Phys. B500 (1997) 255; hep-ph/9612260.

[110] G. Passarino, Nucl. Phys. B574 (2000) 451; hep-ph/9911482.

[111] W. Beenakker, F.A. Berends and A.P. Chapovsky, Nucl. Phys. B573 (2000) 503; hep-ph/0303105.

[112] W. Beenakker et al., Nucl. Phys. B667 (2003) 359; hep-ph/0303105.

[113] M. Beneke, A. P. Chapovsky, A. Signer and G. Zanderighi, Phys. Rev. Lett. 93 (2004) 011602; hep-ph/0312331 and Nucl. Phys. B686 (2004) 205. hep-ph/0401002. 
[114] J. Fujimoto, M. Igarashi, N. Nakazawa, Y. Shimizu and K. Tobimatsu, Suppl. Prog. Theor. Phys. 100 (1990) 1.

[115] G. Bélanger, F. Boudjema, J. Fujimoto, T. Ishikawa, T. Kaneko, K. Kato and Y. Shimizu, hep-ph/0308080.

[116] A. Denner et al., Nucl. Phys. B560 (1999) 33; hep-ph/9904472.

[117] R. G. Stuart, p. 41, in Proceedings of the XXVth Rencontre de Moriond, edited by Tran Thanh Van, Éditions Frontières, Gif-sur-Yvette, 1990.

[118] R. E. Cutkosky, J. Math. Phys. 1 (1960) 429.

[119] G. Passarino and M. Veltman, Nucl. Phys. 160 (1979) 151.

[120] L. M. Brown and R. P. Feynman, Phys. Rev. 85 (1952) 231.

[121] Z. Bern, L. Dixon and D.A. Kosower, Phys. Lett. B302 (1993) 299; Erratum-ibid. B318 (1993) 649; hep-ph/9212308.

[122] G. J. van Oldenborgh, Comput. Phys. Commun. 58 (1991) 1.

[123] J. Fujimoto, Y. Shimizu, K. Kato and Y. Oyanagi, Prog. Theor. Phys. 87 (1992) 1233.

[124] W. Beenakker and A. Denner, Nucl. Phys. B338 (1990) 349.

[125] D. B. Melrose, Il Nuovo Cimento 40A (1965) 181.

W.L. van Neerven and J.A.M. Vermaseren, Phys. Lett. 137 (1984) 241.

[126] P. Nogueira and J.C. Romão, Z. Phys. C60 (1993) 757 .

[127] T. Binoth, J. P. Guillet, G. Heinrich, E. Pilon and C. Schubert, JHEP 10 (2005) 015; hep-ph/0504267.

[128] A. Denner and S. Dittmaier, Nucl. Phys. B734 (2006) 62; hep-ph/0509141.

[129] F. Yuasa et al.,, Proceedings AIHENP 99,hep-ph/0006268. F. Yuasa, D. PerretGallix, S. Kawabata and T. Ishikawa, Nucl. Instrum. and Meth. A389 (1997) 77.

[130] J. Fujimoto, N. Hamaguchi, T. Ishikawa, T. Kaneko, H. Morita, D. Perret-Gallix, A. Tokura and Y. Shimizu, talk given by J. Fujimoto at ACAT05, May 2005, DESY, Zeuthen, Germany.

http://www-zeuthen.desy.de/acat05/talks/Fujimoto. Junpei.3/

talk_fujimoto-1905.ppt. 
[131] R. G. Stuart, Comput. Phys. Commun. 48 (1988) 367.

R. G. Stuart and A. Gongora, Comput. Phys. Commun. 56 (1990) 337.

R. G. Stuart, Comput. Phys. Commun. 85 (1995) 267.

G. Devaraj and R.G. Stuart, Nucl. Phys. B519 (1998) 483.

[132] F. Boudjema, A. Semenov and D. Temes, Phys. Rev. D72 (2005) 055024; hep$\mathrm{ph} / 0507127$.

[133] F. del Aguila and R. Pittau, JHEP 07 (2004) 017; hep-ph/0404120.

[134] W. Giele, E. W. N. Glover and G. Zanderighi, Nucl. Phys. Proc. Suppl. 135 (2004) 275; hep-ph/0407016.

R. K. Ellis, W. T. Giele and G. Zanderighi, hep-ph/0508308.

[135] W. T. Giele and E. W. N. Glover, JHEP 04 (2004) 029; hep-ph/0402152.

[136] A. van Hameren, J. Vollinga and S. Weinzierl, Eur. Phys. J. C41 (2005) 361; hep$\mathrm{ph} / 0502165$.

[137] A. Ferroglia, M. Passera, G. Passarino and S. Uccirati, Nucl. Phys. B650 (2003) 162; hep-ph/0209219.

[138] E. de Doncker, Y. Shimizu, J. Fujimoto and F. Yuasa, Comput. Phys. Commun. 159 (2004) 145.

E. de Doncker, Y. Shimizu, J. Fujimoto, F. Yuasa, K. Kaugars, L. Cucos and J. Van Voorst, Nucl. Instrum. Meth. A534 (2004) 269; hep-ph/0405098.

[139] T. Binoth, G. Heinrich and N. Kauer, Nucl. Phys. B654 (2003) 277; hep$\mathrm{ph} / 0210023$.

[140] Y. Kurihara and T. Kaneko, hep-ph/0503003.

[141] C. Anastasiou and A. Daleo, hep-ph/0511176.

[142] G. Duplancic and B. Nizic, Eur. Phys. J. C35 (2004) 105; hep-ph/0303184.

[143] Y. Kurihara, hep-ph/0504251.

[144] http://minami-home.kek.jp/jf/nlg.html.

[145] F. Bloch and A. Nordsieck, Phys. Rev. 52 (1937) 54.

D.R. Yennie, S.C. Frautchi and H. Suura, Ann. of Phys. 13 (1961) 379.

[146] C. Itzykson and J.B. Zuber, Quantum Field Theory, McGraw-Hill Education, September 1980. 
[147] A. Calogeracos, N. Dombey and A. D. Kennedy, Phys. Lett. (1981) B104 444. J. Frenkel, R. Meuldermans, I. Mohammad and J. C. Taylor, Phys. Lett. B64 (1976) 211 .

[148] R. Gastmans and R. Meuldermans, Nucl. Phys. B63 (1973) 277.

W.J. Marciano and A. Sirlin, Nucl. Phys. B88 (1975) 86.

[149] G. 't Hooft and M. Veltman, Nucl. Phys. B153 (1979) 365.

[150] Y. Kurihara, J. Fujimoto, T. Munehisa and Y. Shimizu, Prog. Theor. Phys. 96 (1996) 1223; hep-ph/9603312.

T. Munehisa, J. Fujimoto, Y. Kurihara and Y. Shimizu, Prog. Theor. Phys. 95 (1996) 375; hep-ph/9603322.

[151] J. Fleischer, A. Leike, T. Riemann, and A. Werthenbach, Eur. Phys. J. C31 (2003) 37 , hep-ph/0302259.

[152] J. Fujimoto and Y. Shimizu, Mod. Phys. Lett. 3A (1988) 581.

[153] W. Beenakker, S. van der Marck, and W. Hollik, Nucl. Phys. B365 (1991) 24-78.

[154] J. Fleischer, J. Fujimoto, T. Ishikawa, A. Leike, T. Riemann, Y. Shimizu, and A. Werthenbach, in Second Symposium on Computational Particle Physics (CPP, Tokyo, 28-30 Nov 2001), KEK Proceedings 2002-11 (2002) (Y. Kurihara, ed.), pp. 153-162; hep-ph/0203220.

[155] J. Fleischer, T. Hahn, W. Hollik, T. Riemann, C. Schappacher, and A. Werthenbach, contribution to the second workshop of the extended ECFA/DESY study Physics and Detectors for a 90 to 800 GeV Linear Collider, 12-15 April 2002, Saint-Malo, France; hep-ph/0202109.

[156] For a nice review on the radiative corrections to $e^{+} e^{-} \rightarrow W^{+} W^{-}$and related issues, see W. Beenakker and A. Denner, Int. J. Mod. Phys. A9 (1994) 4837.

[157] M. Lemoine and M. Veltman, Nucl. Phys. B164 (1980) 445.

M. Böhm, A. Denner, T. Sack, W. Beenakker, F.A. Berends and H. Kuijf, Nucl. Phys. B304 (1988) 463.

J. Fleischer, F. Jegerlehner and M. Zrałek, Z. Phys. C42 (1989) 409.

[158] A. Denner, S. Dittmaier and M. Strobel, Phys. Rev. D53 (1996) 44; hep$\mathrm{ph} / 9507372$.

[159] A. Denner and S. Dittmaier and R. Schuster, Nucl. Phys. B452 (1995) 80; hep$\mathrm{ph} / 9503442$. 
[160] G. Jikia, Nucl. Phys. B494 (1997) 19; hep-ph/9612380.

[161] A. Denner and S. Dittmaier, Nucl. Phys. B398 (1993) 239.

[162] A. Denner and S. Dittmaier, Nucl. Phys. B398 (1993) 265.

[163] A. Denner and T. Hahn, Nucl. Phys. B525 (1998) 27; hep-ph/9711302. 